\title{
Time-Periodic Solutions to the Navier-Stokes Equations
}

\author{
Vom Fachbereich Mathematik \\ der Technischen Universität Darmstadt \\ zur Erlangung der Venia Legendi \\ genehmigte \\ Habilitationsschrift \\ von \\ Dr. rer. nat. Mads Kyed \\ aus Kolding (Dänemark)
}

Darmstadt 2012 



\section{Acknowledgments}

I owe special thanks to Giovanni Paolo Galdi. He became my mentor in the field of Navier-Stokes equations, and without his teachings and guidance over the years I would never have been able to write this thesis. In am deeply indebted to him for inviting me several times to Pittsburgh and for his warm hospitality while I was there.

While I was writing this thesis I was supported by DFG (Deutsche Forschungsgemeinschaft) and JSPS (Japan Society for the Promotion of Science) as a member of the International Research Training Group Darmstadt-Tokyo IRTG 1529. I wish to thank all members of the group for creating an excellent atmosphere for mathematical research. In particular, I would like to thank Matthias Hieber and Reinhard Farwig for their support. I am also grateful to Yoshihiro Shibata for inviting me to stay at Waseda University in Tokyo during the autumn of 2011, his warm hospitality and many fruitful discussions. During my stay at Waseda University I enjoyed several beneficial discussions with Masao Yamazaki. I also wish thank Yoshikazu Giga for taking time to see me at Tokyo University, where I had the opportunity to discuss some of the results in this work with him. 



\section{Contents}

1 Introduction $\quad \mathbf{1}$

1.1 Time-periodic Navier-Stokes equations . . . . . . . . . . . . . . . 1

1.2 History . . . . . . . . . . . . . . . . . 3

1.3 Time-periodic flow past a body . . . . . . . . . . . . . . . 8

1.4 Time-periodic framework . . . . . . . . . . . . . . . . . . . . 10

1.5 Decomposition . . . . . . . . . . . . . . . . . 11

1.6 Asymptotic structure . . . . . . . . . . . . . . . . . 13

2 Notation and preliminaries 17

2.1 Basic notation . . . . . . . . . . . . . . . . 17

2.2 Topological groups and the Fourier transform . . . . . . . . . . . . . 18

2.3 Function spaces . . . . . . . . . . . . . . . . . . . . . . . . . . 19

2.3 .1 Sobolev spaces . . . . . . . . . . . . . . . . . . . 19

2.3 .2 Sobolev embedding . . . . . . . . . . . . . . . . . 20

2.3.3 Helmholtz-Weyl decomposition . . . . . . . . . . . . . . . . . 21

2.3.4 Function spaces involving time . . . . . . . . . . . . . . . 22

2.4 Multiplier theory . . . . . . . . . . . . . . . . . . . . . . . . 22

3 Linear theory 23

3.1 Fourier series expansion in time . . . . . . . . . . . . . . . . . 24

3.2 Reformulation on a group . . . . . . . . . . . . . . . . 27

3.2 .1 Differentiable structure . . . . . . . . . . . . . . 27

3.2 .2 Function spaces . . . . . . . . . . . . . . . . . . . 28

3.2 .3 Fourier transform . . . . . . . . . . . . . . . . . . . 33

3.2 .4 Reformulation . . . . . . . . . . . . . . . . . 34

3.3 Representation formula . . . . . . . . . . . . . . . . . . 35

3.4 Transference of multipliers . . . . . . . . . . . . . . . . . 36

3.5 Time averaging . . . . . . . . . . . . . . . . . . . . . . 41

3.6 Maximal regularity . . . . . . . . . . . . . . . . . . . . . 43

3.7 Flow past a rotating body . . . . . . . . . . . . . . . 53

$\begin{array}{lll}4 & \text { Strong solutions } & \mathbf{6 1}\end{array}$

4.1 Existence . . . . . . . . . . . . . . . . . . . . . 61

4.2 Regularity . . . . . . . . . . . . . . . . . 66

4.3 Energy equation . . . . . . . . . . . . . . . . . . . 72

4.4 Uniqueness . . . . . . . . . . . . . . . . . . . . . . . 74 
Contents

5 Asymptotic structure $\quad 83$

5.1 Asymptotic expansion . . . . . . . . . . . . . . . . . 83

5.2 Energy . . . . . . . . . . . . . . . . . . . . . . . . . . . . 99

5.3 Flow past a rotating body . . . . . . . . . . . . . . . . . . . 102

6 Weak solutions 109

6.1 Weak formulation on the torus group . . . . . . . . . . . . . . . . 109

6.1 .1 Function spaces . . . . . . . . . . . . . . . . . . . . . 110

6.1 .2 Weak formulation . . . . . . . . . . . . . . . 111

6.2 Time-averaging projection . . . . . . . . . . . . . . . . 111

6.3 Existence . . . . . . . . . . . . . . . . . . . . . . . . . 112

6.4 Regularity properties . . . . . . . . . . . . . . . . . . 130

6.5 Existence of a pressure . . . . . . . . . . . . . . . . . . 133

$\begin{array}{lr}\text { Bibliography } & 143\end{array}$

$\begin{array}{lr}\text { Index } & 148\end{array}$ 


\section{Introduction}

\subsection{Time-periodic Navier-Stokes equations}

The flow of an incompressible, viscous, three-dimensional, Newtonian fluid in a domain $\Omega \subset \mathbb{R}^{3}$ is described in a time interval $(0, T)$ by the Navier-Stokes equations

$$
\begin{cases}\partial_{t} \mathfrak{u}-\Delta \mathfrak{u}+\nabla \mathfrak{p}+\mathfrak{u} \cdot \nabla \mathfrak{u}=f & \text { in } \Omega \times(0, T), \\ \operatorname{div} \mathfrak{u}=0 & \text { in } \Omega \times(0, T),\end{cases}
$$

where $\mathfrak{u}: \Omega \times(0, T) \rightarrow \mathbb{R}^{3}$ denotes the Eulerian velocity field, $\mathfrak{p}: \Omega \times(0, T) \rightarrow \mathbb{R}$ the corresponding pressure term, and $f: \Omega \times(0, T) \rightarrow \mathbb{R}^{3}$ an external force. If $\Omega$ has a boundary, (1.1) should be supplemented with appropriate boundary conditions. In case

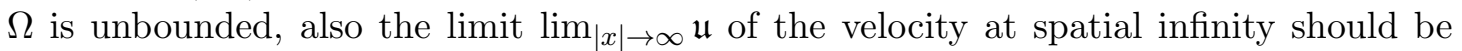
prescribed. For simplicity, in (1.1) both the density and viscosity constant have been put equal to 1 .

To date, the mathematical analysis of (1.1) has been focused primarily on the corresponding initial-value problem, that is, the problem of determining for a prescribed force $f$ and initial value $\mathfrak{u}_{0}$ a solution $(\mathfrak{u}, \mathfrak{p})$ such that $\mathfrak{u}(\cdot, 0)=\mathfrak{u}_{0}$. In addition, also the steady-state problem has been intensively studied, that is, the problem of finding a time-independent solution $(\mathfrak{u}, \mathfrak{p})$ corresponding to a time-independent force $f$. In this work, the time-periodic problem is investigated. More specifically, given a force that is periodic in time with period $\mathcal{T}$,

$$
f: \Omega \times \mathbb{R} \rightarrow \mathbb{R}^{3}, \quad \forall(x, t) \in \Omega \times \mathbb{R}: f(x, t)=f(x, t+\mathcal{T}),
$$

existence and structural properties of $\mathcal{T}$-time-periodic solutions

$$
\begin{array}{lll}
\mathfrak{u}: \Omega \times \mathbb{R} \rightarrow \mathbb{R}^{3}, & \forall(x, t) \in \Omega \times \mathbb{R}: \mathfrak{u}(x, t)=\mathfrak{u}(x, t+\mathcal{T}), \\
\mathfrak{p}: \Omega \times \mathbb{R} \rightarrow \mathbb{R}, & \forall(x, t) \in \Omega \times \mathbb{R}: \mathfrak{p}(x, t)=\mathfrak{p}(x, t+\mathcal{T})
\end{array}
$$

are investigated.

To understand the challenges of the time-periodic problem it is worth comparing it to the initial-value and steady-state problem. On one hand, it resembles the steady-state problem in that no initial value is prescribed. On the other hand, it is a time-dependent problem, so it would be natural to treat it as an evolution equation. Currently, most treatments of the time-periodic problem are based on the latter viewpoint. Typically an arbitrary initial value is introduced and the problem then analyzed as an initial-value problem with the goal of a posteriori showing existence of one initial value that yields a time-periodic solution. Such an investigation is necessarily carried out in a functional 


\section{Introduction}

analytic setting dictated by the characteristics of the initial-value problem. In fact, as will be described further below, with few exceptions such settings are the foundation in most contributions to the time-periodic problem to date.

One of the main purposes of this work is to develop a functional analytic framework that is optimal with respect to the characteristics of the time-periodic problem. The intention is to construct function spaces with respect to which the linear operator corresponding to the time-periodic problem enjoys what is referred to as "maximal regularity". More specifically, for a prescribed time period $\mathcal{T}$ the linearized problem will be formulated in terms of a linear operator

$$
A: \mathrm{X}^{q} \rightarrow L^{q}(\Omega \times(0, \mathcal{T}))^{3}
$$

with the property that $(u, \mathfrak{p}) \in \mathrm{X}^{q}$ is a time-periodic solution to the linearization of (1.1) corresponding to data $f:=A(u, \mathfrak{p})$. The goal is then to identify the particular space $\mathrm{X}^{q}$ with the property that $A$ is a homeomorphism. In this case, $A$ is said to have maximal regularity. Functional analytic frameworks with maximal regularity are available for both the steady-state and initial-value Navier-Stokes problem, but till now have not been identified in the time-periodic case.

A classical application of a maximal regularity framework is to show existence of a strong solution to the original nonlinear problem via the contraction mapping principle. In the following, this will done for the time-periodic problem in the whole-space. Such an existence result is already known in the case the prescribed velocity $\mathfrak{u}_{\infty}:=\lim _{|x| \rightarrow \infty} \mathfrak{u}$ at spatial infinity is 0 . In this work, focus will therefore be on the case $\mathfrak{u}_{\infty} \neq 0$. By employing the maximal regularity framework, one avoids introducing an initial value as mentioned above.

As another important feature, the maximal regularity framework in an $L^{q}$-setting also provides the means to extract valuable information on the asymptotic structure at spatial infinity of the strong solutions. As one of the main results in this work, an asymptotic expansion of the strong solution is obtained. More precisely, it will be shown that

$$
\mathfrak{u}(x, t)-\mathfrak{u}_{\infty}=\Gamma(x) \cdot \alpha+\mathscr{R}(x, t),
$$

where $\Gamma: \mathbb{R}^{3} \rightarrow \mathbb{R}^{3 \times 3}$ is an explicitly known so-called asymptotic profile, $\alpha \in \mathbb{R}^{3}$ a constant that can be computed in terms of the data, and $\mathscr{R}(x, t)$ a remainder term that decays faster than $\Gamma(x)$ as $|x| \rightarrow \infty$. Interestingly, the asymptotic profile turns out to be the same as in the steady-state case, namely the Oseen fundamental solution when $\mathfrak{u}_{\infty} \neq 0$. An estimate of the remainder term will be given both in a pointwise sense and in the sense of summability. The latter estimate is used to derive important information on the kinetic energy of the fluid flow corresponding to the solution.

One can also observe in the theory of weak solutions that the classical setting for the initial-value problem is not optimally suited for the time-periodic problem. To illustrate this, consider the classical function space used to show existence of weak solutions $\mathfrak{u}$ to the initial-value problem corresponding to $(1.1)$, namely the space ${ }^{1}$

$$
L^{2}\left(0, T ; D_{0}^{1,2}(\Omega)^{3}\right) \cap L^{\infty}\left(0, T ; L^{2}(\Omega)^{3}\right) .
$$

\footnotetext{
${ }^{1}$ See Chapter 2 for the definition of the function spaces.
} 
A weak solution to the initial-value problem in the class (1.4) is called a Leray-Hopf solution. The standard techniques used to establish existence of a Leray-Hopf weak solution are based on an a priori bound of the solution in the space above. While an a priori estimate of a $\mathcal{T}$-time-periodic solution to $(1.1)$ in the space $L^{2}\left(0, \mathcal{T} ; D_{0}^{1,2}(\Omega)^{3}\right)$ can easily be obtained by the same methods, the absence of an initial value precludes a bound in the norm of $L^{\infty}\left(0, \mathcal{T} ; L^{2}(\Omega)^{3}\right)$. The inability to establish an a priori bound in $L^{\infty}\left(0, \mathcal{T} ; L^{2}(\Omega)^{3}\right)$ is by no means surprising though. It is namely well-known that a steady-state solution to (1.1) in general does not belong to $L^{2}(\Omega)^{3}$. Since a steady state is trivially time-periodic, time-periodic solutions therefore cannot in general belong to $L^{\infty}\left(0, \mathcal{T} ; L^{2}(\Omega)^{3}\right)$. This observation is somewhat puzzling. It implies that a weak time-periodic solution at the outset has less time regularity than a weak Leray-Hopf solution to the initial-value problem. From a heuristic point of view, this is certainly not expected. One might therefore question whether there is a setting better suited than (1.4) for investigation of weak time-periodic solutions.

Motivated by this question, in this work a modified functional analytic setting for the weak theory is presented. The new approach accommodates for the special characteristics of the time-periodic problem. As a merit hereof, it will be possible to show that weak solutions to the time-periodic problem do in a certain sense, after subtraction of a possible steady-state part, belong to $L^{\infty}\left(0, T ; L^{2}(\Omega)^{3}\right)$. This property is not only important in the context of regularity, but also yields significant information on the kinetic energy of the fluid flow corresponding to a weak solution.

\subsection{History}

Starting with the celebrated works [41] and [42] of LERAY, a vast amount of research has gone into investigation of the steady-state and initial-value Navier-Stokes problem. LERAY was able to solve these problems in a weak sense, but several important questions for both the steady-state and initial-value problem remain open till this date. For a comprehensive overview of known results and open problems, the reader is referred to the monograph [21], the papers [19] and [60], and all references cited there. Below, an overview of results specifically concerning the time-periodic Navier-Stokes system is given.

The weak solution to the initial-value problem established by LERAY in [42] belongs to the class (1.4). Such a solution is typically referred to as a Leray-Hopf weak solution. As described in Section 1.1, a weak solution to the time-periodic problem is more appropriately classified as an element of the larger space $L^{2}\left(0, T ; D_{0}^{1,2}(\Omega)^{3}\right)$. If a solution $\mathfrak{u}$ possess a certain amount of additional regularity, at least $\partial_{t} \mathfrak{u}$ and $\nabla^{2} \mathfrak{u}$ should be summable to some degree, we call it a strong solution.

The study of the time-periodic problem was initiated by SERRIN in [52]. SERRIN argues that for a time-periodic force $f$ and any initial value the solution $\mathfrak{u}(x, t)$ to the corresponding initial-value problem converges as $t \rightarrow \infty$ to some state which, when considered as an initial value in the initial-value problem, yields a time-periodic solution. The rationale behind SERRIN postulate is that $\mathfrak{u}(x, \mathcal{T} n), \mathcal{T}$ being the time period of $f$, 


\section{Introduction}

converges as $n \rightarrow \infty$ to a state on a periodical orbit. The result of SERRIN is based on a number of assumptions concerning the resolvability of the initial-value problem which, even today, is known only to hold under special circumstances, for example, the domain in his case must be bounded. Although SERRIN did not present any rigorous proofs in his very short paper, the method described to obtain a time-periodic solution has been used by a number of authors later on. Below, we shall refer to this method as Serrin's method.

Another approach was introduced a year later by Yudovich in [62]. Yudovich considers the Poincaré map that takes an initial value into the state corresponding to the solution of the corresponding initial-value problem evaluated at time $\mathcal{T}$, where $\mathcal{T}$ is the period of the prescribed force $f$. A time-periodic solution is then identified as a fixed point of this Poincaré map. The same technique was described around the same time, and seemingly independently, by PRODI in [49]. As in the case of SERRIN's article, neither the paper by YUDOVICH nor the paper by PRODI contain rigorous proofs, yet their ideas have subsequently been used extensively by several other authors. We shall refer to their method as the Prodi-Yudovich method.

The first to rigorously prove existence of a time-periodic solution was Prouse in [51], where existence of a time-periodic solution in a bounded two-dimensional domain was shown. In [50] the same author showed existence also in three- and higher-dimensional bounded domains. The proofs of Prouse rely on the Prodi-Yudovich method. To treat the initial-value problem, Prouse applied a Galerkin method technique, that is, an approximation of the problem in finite-dimensional function spaces. Originally introduced by Hopf in [32], today this technique is part of the standard literature on the NavierStokes problem. Applying the fixed-point iteration described by Prodi and YUdovich on the finite dimensional level, PROUSE showed existence of a weak time-periodic solution for arbitrarily "large" data.

Existence of strong time-periodic solutions was established a few years later by KANIEL and Shinbrot in [34]. Based on Serrin's method, Kaniel and Shinbrot showed for a three-dimensional bounded domain the existence of a strong solution when the data is assumed sufficiently "small".

Although KANIEL and SHINBRot claim their result also holds for two-dimensional bounded domains, TAKESHITA treated rigorously the two-dimensional case in [55]. In fact, TAKESHITA established existence of a strong time-periodic solution without any restriction on the "size" of the data. Such a result was already known for the initialvalue problem in two-dimensional domain.

The existence theory for the time-periodic problem in bounded domains was supplemented by Morimoto in [48] and Miyakawa and Tеramoto in [47] who investigated time-dependent domains, both two- and three-dimensional, using the Prodi-Yudovich method. A stability result in such a setting was obtained by Teramoto in [57].

At this point in time, the theory for the time-periodic problem in bounded domains had reached a level where everything that could be proved for the initial-value problem could also be shown for the time-periodic problem. However, no results had been obtained for 
unbounded domain. Recall that in the case of an unbounded domain the condition

$$
\lim _{|x| \rightarrow \infty} \mathfrak{u}(x, t)=\mathfrak{u}_{\infty}
$$

must be added to the system (1.1).

The first result for unbounded domains is due to Maremonti. In [44] Maremonti treated the time-periodic problem in the whole-space $\mathbb{R}^{3}$ with $\mathfrak{u}_{\infty}=0$. Based on Serrin's method, he showed existence of a strong solution for "small" data. In addition, MAREMONTI also established stability of the solution. Stability properties in fact follow in a natural way when Serrin's method is employed. The solution found in [44] belongs to the class $L^{\infty}\left(0, T ; L_{\sigma}^{3}\left(\mathbb{R}^{3}\right)^{3}\right)$. Since a steady-state solution to the same whole-space problem in general does not belong to $L_{\sigma}^{3}\left(\mathbb{R}^{3}\right)$, and since a steady state is also time-periodic, such a result can only be obtained if a structural condition is imposed on the data. In [44] it is assumed that $f=\operatorname{curl} \psi$ for an appropriate vector field $\psi$.

Similar results as in [44] were shown around the same time for the three-dimensional half-space by the same author in [45]. A further extension of the result to other unbounded domains with non-empty boundaries is more complicated though.

The time-periodic problem in more general unbounded domains with $\mathfrak{u}_{\infty}=0$ was investigated by MAREMONTI and PADULA in [46]. In [46] existence of a weak solution in arbitrary domains, including also the two-dimensional case, is established. The proof is based on the "invading domain" technique (introduced by HEYwOOD in [30]) combined with the method of Prodi-Yudovich. More precisely, the problem is first solved in a sequence of bounded domains using the Prodi-Yudovich method. By letting the sequence tend to the original unbounded domain, one obtains the final solution as the limit of the corresponding sequence of solutions in the bounded domains. Additional regularity is established under the condition that the data is sufficiently "small". Assuming that both the force and the domain possess a certain amount of symmetry, MAREMONTI and PADULA further showed in the three-dimensional case the existence of a strong solution in the function space $L^{\infty}\left(0, T ; L_{\sigma}^{3}(\Omega)\right)$. For this part, they reverted to Serrin's method. As in the case of the three-dimensional whole-space, such a result cannot be shown for arbitrary data without any structural condition imposed. In other words, the symmetric structure of the data and the domain is crucial for this result. It is important to observe that a uniformly bounded (in time) $L^{3}$-norm is a significant information on the decay of the solution at spatial infinity. Utilizing this information, MAREMONTI and PADULA established uniqueness of the solution in the class of strong solutions. Although the half-space does not satisfy the symmetry conditions, MAREMONTI and PADUla claim the result can easily be extended to the half-space.

A new important contribution to the time-periodic problem was given by KozONO and NAKAO in [36] who, for the first time, presented a direct representation formula for the solution. Although KOzONO and NAKAO established the same type of result that was already known from [44] and [45], namely the existence of a strong solution in the three-dimensional whole- or half-space for $\mathfrak{u}_{\infty}=0$ and sufficiently "small" data, their direct method was a significant improvement. More precisely, KOzONO and NAKAO observed that the solution to the time-periodic Stokes problem can be expressed as a 


\section{Introduction}

convolution integral with the Stokes semi-group over the whole real line $\mathbb{R}$, that is,

$$
\mathfrak{u}(x, t)=\int_{-\infty}^{t} \mathrm{e}^{-(t-s) A} \mathcal{P}_{H} f(s) \mathrm{d} s
$$

where $A$ denotes the Stokes operator and $\mathcal{P}_{H}$ the Helmholtz projection. Based on properties of the Stokes semi-group, a solution to the time-periodic Navier-Stokes system can then be obtained by successive approximation. An advantage of this approach is that the representation formula

$$
\mathfrak{u}(x, t)=\int_{-\infty}^{t} \mathrm{e}^{-(t-s) A}\left(\mathcal{P}_{H} f(s)-\mathcal{P}_{H}(\mathfrak{u} \cdot \nabla \mathfrak{u})\right) \mathrm{d} s
$$

for the solution follows. Compared to the methods of Serrin and Prodi-Yudovich, the approach of Kozono and NAKAO is much more direct. Similar to [44] and [45], KoZONO and NAKAO obtained a strong solution in the space $L^{\infty}\left(0, T ; L_{\sigma}^{3}(\Omega)\right)$. As already mentioned, such a result necessarily requires a structural condition imposed on the data $f$, which in [36] is $\mathcal{P}_{H} f(s)=A^{\delta} g(s)$ for some appropriate constant $\delta>0$ and function $g: \mathbb{R} \rightarrow D\left(A^{\delta}\right)$. KOZONO and NAKAO also showed uniqueness of the strong solution in the class of strong solutions. Although the results in [36] in the three-dimensional case hold only for the whole- and half-space, in dimension $n \geq 4$ they are established for an exterior domain.

A few years later, Yamazaki was able to employ in [61] the Kozono-Nakao method to a three-dimensional exterior domain with $\mathfrak{u}_{\infty}=0$. The new idea of YAMAZAKI was to use the space $L^{\infty}\left(0, T ; L_{\sigma}^{3, \infty}(\Omega)\right)$ instead of $L^{\infty}\left(0, T ; L_{\sigma}^{3}(\Omega)\right)$. Here, $L_{\sigma}^{3, \infty}(\Omega)$ denotes the Lorentz space weak- $L^{3}(\Omega)$. Since a steady-state solution belongs to $L_{\sigma}^{3, \infty}(\Omega)$ for a large and natural class of data on the form $f=\operatorname{div} F$, it seems more appropriate to study the time-periodic problem in an $L_{\sigma}^{3, \infty}(\Omega)$-setting rather than in $L_{\sigma}^{3}(\Omega)$. Moreover, in the case of an exterior domain the integral in (1.6) converges in $L_{\sigma}^{3, \infty}(\Omega)$, but not in $L_{\sigma}^{3}(\Omega)$. This explains why Yamazaki, in contrast to Kozono and NAKAO in [36], was able to treat three-dimensional exterior domains. More specifically, YAMAZAKI showed existence of a so-called mild solution in $L^{\infty}\left(0, T ; L_{\sigma}^{3, \infty}(\Omega)\right)$ for sufficiently "small" data in the case that $\Omega$ is either an exterior domain, the whole-space, or the half-space in $\mathbb{R}^{n}$. Although not the same, a mild solution is, in the sense of regularity, close to a weak solution. Interestingly, YAMAZAKI also established uniqueness of a mild solution within the quite large class of mild solutions, provided still the magnitude of the data is "small".

In a further development, GALDI and SoHR investigated in [14] the time-periodic problem in three-dimensional exterior domains and $\mathfrak{u}_{\infty}=0$. GALDI and SoHR showed existence of strong solutions for sufficiently "small" data. These solutions are more regular than the mild solutions established by YAMAZAKI in [61], whence, since both results require a restriction on the "size" of the data, their result constituted an important improvement. Similar to [61], GALDI and SoHR introduced a function space that contains 
the steady states for a large class of data on the form $f=\operatorname{div} F$. More precisely, the result in [14] is based on an investigation of solutions with values, for each time $t$, in the weighted space

$$
\mathcal{X}(\Omega):=\left\{v \in L_{l o c}^{1}(\Omega)^{3}\left|\sup _{x \in \Omega}(|x|+1)\right| v(x) \mid<\infty\right\} .
$$

Observe that for a three-dimensional exterior domain $\Omega$ the space $\mathcal{X}(\Omega)$ is slightly larger than $L^{3}(\Omega)^{3}$, but smaller than the space $L^{3, \infty}(\Omega)^{3}$ used by YAMAZAKI in [61]. GaLdi and SoHR established existence of a time-periodic solution in $L^{\infty}(0, T ; \mathcal{X}(\Omega))$, provided, as already mentioned, the data is sufficiently "small". Under a similar condition they showed uniqueness in a rather large subspace of $L^{\infty}(0, T ; \mathcal{X}(\Omega))$. Their proof is based on Serrin's method. An immediate advantage of their functional analytic setting is the information obtained on the decay of the solution at spatial infinity, namely a decay rate uniformly in time of $1 /|x|$ as $|x| \rightarrow \infty$. At the time GALDI and SoHr published their paper, this was the best result available on the asymptotic structure at spatial infinity of a time-periodic solution.

Recently, the asymptotic profile of the solution was identified by KANG, MiUrA, and Tsai in [33]. More precisely, KAng, MiURA, and TSAI showed for a three-dimensional exterior domain and $\mathfrak{u}_{\infty}=0$ that a time-periodic solution which belongs to the space $L^{\infty}\left(0, T ; L_{\sigma}^{3, \infty}(\Omega)\right)$ tends at spatial infinity to a so-called Landau solution, provided the data is sufficiently "small". We shall discuss this result in more detail in Section 1.6.

The asymptotic structure of the solution is closely connected with questions concerning the kinetic energy of the corresponding fluid flow. One important question in this respect is simply whether the kinetic energy is finite. In the very recent work [53], Silvestre has established existence of strong solutions with finite kinetic energy under suitable assumptions on the data. More specifically, Silvestre investigates the whole-space problem with $\mathfrak{u}_{\infty}=0$. Assuming the data $f$ is sufficiently small, has compact support, and satisfies $\int_{\mathbb{R}^{3}} f(x, t) \mathrm{d} x=0$ for all times $t$, the existence of a solution $\mathfrak{u} \in L^{2}\left(0, T ; L^{2}\left(\mathbb{R}^{3}\right)^{3}\right)$ is shown.

All results above concern the classical Navier-Stokes system as described in (1.1), which models the flow of a viscous fluid in $\Omega$ under the action of an outer force $f$. Many fluid flow problems, however, concern the motion of a body in a fluid. In such a case the fluid domain is the exterior of some bounded domain $\mathcal{B}(t)$, which models the body and hence moves in time. The fluid domain is then also time-dependent. In order to mathematically investigate this problem, it is necessary to rewrite the equations of motion for the fluid, that is, the Navier-Stokes system, in a frame of reference attached to the body. A system is thereby obtained in a fixed (in time) domain, but with additional terms appearing in the equations of motion. As a good reference for this type of problem, the reader is referred to [20]. If the motion of the body is time periodic, the equations of motion in the new frame of reference will have time-periodic coefficients and boundary conditions. In other words, one then obtains a time-periodic Navier-Stokes problem in a frame of reference that is given by the motion of the body, and which is not necessarily an inertial frame. This special type of time-periodic Navier-Stokes problem was investigated for the first time by Galdi and Silvestre in [25]. Galdi and Silvestre assumed a 


\section{Introduction}

prescribed motion of the body given by a time-periodic translational velocity $\xi(t)$ and time-periodic angular velocity $\omega(t)$, which yields

$$
\begin{cases}\partial_{t} \mathfrak{u}-\Delta \mathfrak{u}+\nabla \mathfrak{p}+\mathfrak{u} \cdot \nabla \mathfrak{u}-(\xi+\omega \wedge x) \cdot \nabla \mathfrak{u}+\omega \wedge \mathfrak{u}=f & \text { in } \Omega \times(0, T), \\ \operatorname{div} \mathfrak{u}=0 & \text { in } \Omega \times(0, T), \\ \mathfrak{u}=\xi+\omega \wedge x & \text { on } \partial \Omega \times(0, T), \\ \lim _{|x| \rightarrow \infty} \mathfrak{u}(x, t)=0 & \end{cases}
$$

as equations of motion in a frame attached to the body. Here, $\Omega$ is a three-dimensional exterior domain. Using a combination of an "invading domain" technique and the ProuseYudovich method, GALDI and SiLvestre showed existence of a weak time-periodic solution to (1.7). In addition, existence of a strong solution is established in [25] under the assumption that the data $\xi(t), \omega(t)$, and $f$ are sufficiently "small". In a further investigation of this problem, the same authors showed a few years later in [27] a similar result for the unconstrained motion of a body under the influence of a time-periodic body force, thereby extending a famous result of WeInBERGER $[58,59]$ to the time-periodic case.

\subsection{Time-periodic flow past a body}

The main focus in this work will be the time-periodic Navier-Stokes system in an unbounded domain with a prescribed non-zero constant velocity $\mathfrak{u}_{\infty} \neq 0$ at spatial infinity. As described in the previous section, few results are available for this particular case.

In a three-dimensional exterior domain, the Navier-Stokes system with a prescribed constant velocity $\mathfrak{u}_{\infty} \neq 0$ at spatial infinity describes the flow of a viscous, incompressible fluid past a rigid body moving with velocity $-\mathfrak{u}_{\infty}$. If, in addition to the condition $\mathfrak{u}_{\infty} \neq 0$, the data of the system is time periodic, we refer to a time-periodic solution as a timeperiodic flow past a body. If $\mathfrak{u}_{\infty}=0$, the solution is referred to as a time-periodic flow around a body.

The Navier-Stokes system corresponding to a flow past or around a body is

$$
\begin{cases}\partial_{t} \mathfrak{u}-\Delta \mathfrak{u}+\nabla \mathfrak{p}+\mathfrak{u} \cdot \nabla \mathfrak{u}=f & \text { in } \Omega \times(0, T) \\ \operatorname{div} \mathfrak{u}=0 & \text { in } \Omega \times(0, T) \\ \mathfrak{u}=\mathfrak{u}_{*} & \text { on } \partial \Omega \times(0, T) \\ \lim _{|x| \rightarrow \infty} \mathfrak{u}(x, t)=\mathfrak{u}_{\infty}, & \end{cases}
$$

where $\Omega \subset \mathbb{R}^{3}$ is an exterior domain. The outer forces in the system are given in terms of the force $f$ acting on fluid, and the momentum flux through the body's boundary determined by $\mathfrak{u}_{*}$ and $\mathfrak{u}_{\infty}$. A common scenario is the self-propelled motion of an object like an airplane or submarine, in which case the body would be driven solely via momentum flux through the boundary. If the driving mechanism is of a time-periodic nature, 
for example a rotating propeller, the corresponding data $\mathfrak{u}_{*}$ in the model would be time periodic. Thus, time-periodic flows past or around rigid bodies appear in connection with several important models in engineering and physics.

Without loss of generality, we assume that $\mathfrak{u}_{\infty}$ is directed along the $\mathrm{e}_{1}$-axis, that is $\mathfrak{u}_{\infty}=\lambda \mathrm{e}_{1}$ for some $\lambda \in \mathbb{R}$. If we instead of $\mathfrak{u}$ consider the relative velocity $u:=\mathfrak{u}-\mathfrak{u}_{\infty}$, we obtain the equivalent system

$$
\begin{cases}\partial_{t} u-\Delta u-\lambda \partial_{1} u+\nabla \mathfrak{p}+u \cdot \nabla u=f & \text { in } \Omega \times(0, T), \\ \operatorname{div} u=0 & \text { in } \Omega \times(0, T), \\ u=\mathfrak{u}_{*}-\lambda \mathrm{e}_{1} & \text { on } \partial \Omega \times(0, T), \\ \lim _{|x| \rightarrow \infty} u(x, t)=0 . & \end{cases}
$$

In this formulation, the difference between a flow past a body and a flow around a body is the appearance of the so-called drift term $\lambda \partial_{1} u$ in the field equation. Although this term is of lower order, it has a significant impact on the asymptotic structure at spatial infinity of a corresponding solution.

Many important properties of (1.9), in particular the asymptotic structure of the solutions at spatial infinity, can be derived by studying the corresponding whole-space problem

$$
\begin{cases}\partial_{t} u-\Delta u-\lambda \partial_{1} u+\nabla \mathfrak{p}+u \cdot \nabla u=f & \text { in } \mathbb{R}^{3} \times(0, T), \\ \operatorname{div} u=0 & \text { in } \mathbb{R}^{3} \times(0, T), \\ \lim _{|x| \rightarrow \infty} u(x, t)=0 . & \end{cases}
$$

The main objective in this work is to investigate time-periodic solutions to (1.10) for a given time-periodic right-hand side $f$. One of the goals is to establish existence of solutions and identify the asymptotic structure of these solutions at spatial infinity.

There are currently few results available for time-periodic solutions to (1.10) in the case $\lambda \neq 0$. Due to the term $\lambda \partial_{1} u$, the linearization of the system is the so-called Oseen system. In contrast, in the case $\mathfrak{u}_{\infty}=0$, that is, $\lambda=0$, one obtains the Stokes system. Most of the results mentioned in Section 1.2 concern the case $\mathfrak{u}_{\infty}=0$ and rely on properties of the Stokes fundamental solution. These methods can therefore not easily be adapted to the case $\mathfrak{u}_{\infty} \neq 0$. Only the approach of Galdi and Silvestre from [25] can be modified to show existence of a time-periodic solution in the case $\mathfrak{u}_{\infty} \neq 0$. Their approach yields existence of a weak solution in the space $L^{2}\left(0, \mathcal{T} ; D_{0}^{1,2}\left(\mathbb{R}^{3}\right)^{3}\right)$, where $\mathcal{T}$ denotes the time period of the data $f$.

In this work, time-periodic solutions to (1.10) are investigated. The following results are obtained: In Theorem 3.6.9 maximal regularity in a general $L^{q}$-setting is established for the linearized system. The existence of a strong solution in the maximal regularity space is then shown in Theorem 4.1.5 for "small" data in the case $\lambda \neq 0$. A solution is referred to as strong if all derivatives up to the order appearing in (1.10) are summable in appropriate $L^{q}\left(\mathbb{R}^{3}\right)$-spaces. In Theorem 4.2.1 such solutions are shown to be "as regular" as the data. In addition, uniqueness in a fairly large class is established in 


\section{Introduction}

Theorem 4.4.5. In Theorem 5.1.6 and Theorem 5.2.2 an asymptotic expansion of the solution as $|x| \rightarrow \infty$ is carried out. In particular, an asymptotic profile is completely identified. Finally, in Theorem 6.3.1 the existence of a weak $\mathcal{T}$-time-periodic solution in the space $L^{2}\left(0, \mathcal{T} ; D_{0}^{1,2}\left(\mathbb{R}^{3}\right)^{3}\right)$ is established for arbitrarily "large" data that are $\mathcal{T}$ periodic in time. In addition, it is shown that a certain part of this weak solution belongs to $L^{\infty}\left(0, \mathcal{T} ; L^{2}\left(\mathbb{R}^{3}\right)^{3}\right)$. Since elements in $L^{2}\left(0, \mathcal{T} ; D_{0}^{1,2}\left(\mathbb{R}^{3}\right)^{3}\right)$ are not defined in a pointwise sense, it is not possible at the outset to determine, or even define, $\mathcal{T}$-timeperiodicity for the functions, or strictly speaking equivalence classes of functions, in this space. To conclude that a weak solution is in fact time-periodic, it is therefore important to establish additional regularity in time. This is done in Theorem 6.4.3, where it is shown that the weak solution is continuous in an appropriate topology. Moreover, the existence of a pressure term is shown in Theorem 6.5.1. Provided the data has additional regularity, a more regular pressure is constructed in Theorem 6.5.3.

For convenience, we will always assume $\lambda$ to be non-negative in (1.10). We shall therefore only distinguish between the case $\lambda=0$ and $\lambda>0$. All results obtained for the case $\lambda>0$, however, are also true for $\lambda<0$.

\subsection{Time-periodic framework}

The methods presented in this work are based on a functional analytic setting of time-periodic functions. While this may seem a very natural approach, most of the contributions mentioned in Section 1.2 rely on the functional analytic setting of an initial-value problem. For example, both the methods of Serrin and Prodi-Yudovich are based on an investigation of the initial-value Navier-Stokes system, and the time-periodic property of the solutions only obtained a posteriori.

For any set $Y$, the time-periodic functions $g: \mathbb{R} \rightarrow Y$ with period $\mathcal{T}$, that is, the functions satisfying $g(t)=g(t+\mathcal{T})$ for all $t \in \mathbb{R}$, can be identified with the functions $g: \mathbb{T} \rightarrow Y$ on the torus group $\mathbb{T}:=\mathbb{R} / \mathcal{T} \mathbb{Z}$. When $\mathbb{T}$ is equipped in the canonical way with a topology and a differentiable structure, the time-periodic Navier-Stokes system can be equivalently formulated in a setting of functions defined on $\mathbb{T}$. The main advantage of such a setting is that all functions are by definition time periodic. The methods introduced in the following are all based on functional analytical framework in which the functions are defined on the torus group.

In Chapter 6 weak solutions to the time-periodic Navier-Stokes problem are studied in Sobolev spaces $W^{l, s}(\mathbb{T} ; \mathcal{H})$ of $\mathbb{T}$-defined vector fields. Here $\mathcal{H}$ denotes a Hilbert space. In contrast to the classical Sobolev-spaces $W^{l, s}([0, \mathcal{T}] ; \mathcal{H})$ of functions defined on the time-interval $[0, \mathcal{T}]$, the space $W^{l, s}(\mathbb{T} ; \mathcal{H})$ possesses an orthonormal basis of the type $\left\{\mathrm{e}^{i \frac{\mathcal{T}}{2 \pi} k t} \psi_{j} \mid k, j \in \mathbb{Z}\right\}$, where $\left\{\mathrm{e}^{i \frac{\mathcal{T}}{2 \pi} k t} \mid k \in \mathbb{Z}\right\}$ is the set of characters on $\mathbb{T}$, and $\left\{\psi_{j} \mid j \in \mathbb{Z}\right\}$ some basis for $\mathcal{H}$. The main idea behind the approach used in Chapter 6 is to apply a Galerkin approximation based on a sequence of finite spans of these basis vectors.

To identify the maximal regularity function spaces, the group-theoretical approach is taken one step further. In Chapter 3 the time-periodic Navier-Stokes problem is reformulated on the group $G:=\mathbb{R}^{3} \times \mathbb{T}$. By equipping $\mathbb{R}^{3} \times \mathbb{T}$ with the canonical topology 
and differentiable structure from $\mathbb{R}^{3} \times \mathbb{R}$, the time-periodic Navier-Stokes system can be equivalently formulated in a setting of $G$-defined vector fields. Since $G$ is a locally compact abelian group, the Fourier transform $\mathcal{F}_{G}$ on $G$ can then be employed. In fact, the structure of $G$ is such that a Schwartz space $\mathscr{S}(G)$ can be defined in a natural way, and thus also the space $\mathscr{S}^{\prime}(G)$ of tempered distributions. Based on these tools, the linear theory can be studied in terms of $G$-multipliers, that is, Fourier multipliers with respect to the $\mathcal{F}_{G}$ Fourier transform. In connection with a very powerful transference principle for group multipliers (originally introduced by DE LEEUW in [4]) such an abstract formulation turns out to be very useful.

\subsection{Decomposition}

Another important idea that is utilized throughout this work is to decompose the timeperiodic functions into a time-independent part, also referred to as the steady-state part, and a time-dependent part with mean value zero, also referred to as the time-periodic part.

To illustrate the motivation behind the decomposition, consider a $\mathcal{T}$-time-periodic solution $(u, \mathfrak{p})$ to the linear problem

$$
\begin{cases}\partial_{t} u-\Delta u-\lambda \partial_{1} u+\nabla \mathfrak{p}=f & \text { in } \mathbb{R}^{3} \times[0, \mathcal{T}], \\ \operatorname{div} u=0 & \text { in } \mathbb{R}^{3} \times[0, \mathcal{T}], \\ \lim _{|x| \rightarrow \infty} u(x, t)=0 & \end{cases}
$$

with respect to a $\mathcal{T}$-time-periodic right-hand side $f$. Since $u$ is $\mathcal{T}$-time-periodic, it follows that

$$
\int_{0}^{\mathcal{T}} \partial_{t} u \mathrm{~d} t=0
$$

Thus putting

$$
u_{s}:=\frac{1}{\mathcal{T}} \int_{0}^{\mathcal{T}} u \mathrm{~d} t, \quad \mathfrak{p}_{s}:=\frac{1}{\mathcal{T}} \int_{0}^{\mathcal{T}} \mathfrak{p} \mathrm{d} t, \quad f_{s}:=\frac{1}{\mathcal{T}} \int_{0}^{\mathcal{T}} f_{s} \mathrm{~d} t
$$

one obtains, by simply integrating both sides in (1.11) from 0 to $\mathcal{T}$ with respect to time, that

$$
\begin{cases}-\Delta u_{s}-\lambda \partial_{1} u_{s}+\nabla \mathfrak{p}_{s}=f_{s} & \text { in } \mathbb{R}^{3}, \\ \operatorname{div} u_{s}=0 & \text { in } \mathbb{R}^{3} \\ \lim _{|x| \rightarrow \infty} u_{s}(x)=0 & \end{cases}
$$




\section{Introduction}

This means that $\left(u_{s}, \mathfrak{p}_{s}\right)$ is a steady-state solution to (1.11) corresponding to the timeindependent data $f_{s}$. We refer to $\left(u_{s}, \mathfrak{p}_{s}\right)$ as the steady-state part of $(u, \mathfrak{p})$. Put

$$
u_{p}(x, t):=u(x, t)-u_{s}(x), \quad \mathfrak{p}_{p}(x, t):=\mathfrak{p}(x, t)-\mathfrak{p}_{s}(x), \quad f_{p}(x, t):=f(x, t)-f_{s}(x) .
$$

It is clear that the remaining part $\left(u_{p}, \mathfrak{p}_{p}\right)$ is a $\mathcal{T}$-time-periodic solution to (1.11) corresponding to the $\mathcal{T}$-time-periodic data $f_{p}$. We call $\left(u_{p}, \mathfrak{p}_{p}\right)$ the time-periodic part of $(u, \mathfrak{p})$. Observe that

$$
\int_{0}^{\mathcal{T}} u_{p}(x, t) \mathrm{d} t=0, \quad \int_{0}^{\mathcal{T}} \mathfrak{p}_{p}(x, t) \mathrm{d} t=0, \quad \int_{0}^{\mathcal{T}} f_{p}(x, t) \mathrm{d} t=0,
$$

that is, the time-periodic parts have a vanishing mean value with respect to time.

The decomposition described above will play a crucial role in the linear theory in Chapter 3. In particular, it is fundamental to the identification of the space $\mathrm{X}^{q}$ with the maximal regularity property that

$$
A: \mathrm{X}^{q} \rightarrow L^{q}\left(\mathbb{R}^{3} \times[0, \mathcal{T}]\right)^{3}, \quad A(u, \mathfrak{p}):=f
$$

is a homeomorphism, where $A$ denotes the linear operator corresponding to (1.11). By decomposing $(u, \mathfrak{p})$ into $\left(u_{s}, \mathfrak{p}_{s}\right)$ and $\left(u_{p}, \mathfrak{p}_{p}\right)$, and likewise $f$ into $f_{s}$ and $f_{p}$, one can split the operator $A$ into a steady-state part $A_{s}$ and a time-periodic part $A_{p}$ such that

$$
A_{s}\left(u_{s}, \mathfrak{p}_{s}\right)=f_{s}, \quad A_{p}\left(u_{p}, \mathfrak{p}_{p}\right)=f_{p}
$$

The task of establishing maximal regularity is then reduced to identifying function spaces $\mathrm{X}_{s}^{q}$ and $\mathrm{X}_{p}^{q}$ such that both

$$
A_{s}: \mathrm{X}_{s}^{q} \rightarrow L^{q}\left(\mathbb{R}^{3}\right)^{3} \quad \text { and } \quad A_{p}: \mathrm{X}_{p}^{q} \rightarrow L_{0}^{q}\left(\mathbb{R}^{3} \times[0, \mathcal{T}]\right)^{3}
$$

are homeomorphisms, where $L_{0}^{q}\left(\mathbb{R}^{3} \times[0, \mathcal{T}]\right)$ denotes the subspace of $L^{q}\left(\mathbb{R}^{3} \times[0, \mathcal{T}]\right)$ of functions with vanishing mean value with respect to time. In view of (1.14), the space $\mathrm{X}_{p}^{q}$ must also be restricted to vector fields with this property. One can now recognize the operator $A_{s}$ as the classical steady-state Oseen $(\lambda \neq 0)$ or Stokes $(\lambda=0)$ operator. For this operator maximal regularity results are already available. In other words, the space $\mathrm{X}_{s}^{q}$ is well-known. Thus, only the space $\mathrm{X}_{p}^{q}$ needs to be identified. It turns out, as will be clear later, that the group-theoretical multiplier approach described in Section 1.4 works particularly well in settings of vector fields with vanishing mean value with respect to time. Consequently, the space $\mathrm{X}_{p}^{q}$ will be identified with these methods. Finally, the maximal regularity space $\mathrm{X}^{q}$ is then given by $\mathrm{X}_{s}^{q} \times \mathrm{X}_{p}^{q}$.

The existence of strong solutions is established in Chapter 4 by employing the linear theory from Chapter 3. Consequently, also these strong solutions will be elements of the space $\mathrm{X}_{s}^{q} \times \mathrm{X}_{p}^{q}$. More specifically, strong solutions are obtained with a steady-state part and a time-periodic part belonging to different function spaces. In the investigation of the asymptotic structure of the strong solutions at spatial infinity, this information plays 
an important role. It turns out that the time-periodic part decays faster as $|x| \rightarrow \infty$ than the steady-state part. Thus, the asymptotic profile of the solution is determined by the steady-state part in $\mathrm{X}_{s}^{q}$.

The decomposition described above will also be used to study weak time-periodic solutions in Chapter 6. As mentioned in Section 1.1, it is known how to show existence of a weak solution $u$ in the space $L^{2}\left(0, T ; D_{0}^{1,2}\left(\mathbb{R}^{3}\right)^{3}\right)$. Unlike solutions to the corresponding initial-value problem, however, these solutions do not in general belong to $L^{\infty}\left(0, T ; L^{2}\left(\mathbb{R}^{3}\right)^{3}\right)$, which raises the question whether weak solutions to the timeperiodic problem are less regular than corresponding weak solutions to the initial-value problem. The decomposition of $u$ into $u_{s}$ and $u_{p}$ yields an answer to this question. It will namely be shown in Chapter 6 that $u_{p} \in L^{\infty}\left(0, T ; L^{2}\left(\mathbb{R}^{3}\right)^{3}\right)$. Thus, regularity-wise the weak solutions to the time-periodic Navier-Stokes problem are at the outset as good as the weak Leray-Hopf solutions to the initial-value problem.

\subsection{Asymptotic structure}

In order to put the asymptotic expansion in Theorem 5.1.6 and Theorem 5.2.2 properly into context, a brief overview of similar results is presented below.

The investigation of the asymptotic structure of a solution to the steady-state problem

$$
\begin{cases}-\Delta v-\lambda \partial_{1} v+\nabla p=f & \text { in } \mathbb{R}^{3}, \\ \operatorname{div} v=0 & \text { in } \mathbb{R}^{3}, \\ \lim _{|x| \rightarrow \infty} v(x)=0 . & \end{cases}
$$

was initiated by FinN. In the case $\lambda \neq 0$, FinN showed in [12], see also [13], that a solution with sufficient decay at infinity satisfies the asymptotic expansion

$$
v(x)=\Gamma_{\mathrm{O}}^{\lambda}(x) \cdot\left(\int_{\mathbb{R}^{3}} f(x) \mathrm{d} x\right)+\mathscr{R}(x) \quad(\lambda \neq 0),
$$

where $\Gamma_{\mathrm{O}}^{\lambda}$ denotes the so-called Oseen fundamental solution, see also (5.30), and $\mathscr{R}(x)$ a remainder term that decays faster as $|x| \rightarrow \infty$ than $\Gamma_{\mathrm{O}}^{\lambda}(x)$. The result of FinN was later extended by BABENKO, who proved in [2] that the asymptotic expansion is valid even for weak solutions, more specifically for so-called Leray solutions. The proof provided by BABENKO, however, was not complete, and it was not until [15] that a full proof was available; see also [10]. In the case $\lambda=0$, an asymptotic expansion was available only much later, and only for solutions corresponding to "small" data. This result is due to KOROLEV and ŠvERAK, who showed in [35] that a Leray solution to (1.16) satisfies

$$
v(x)=\Gamma_{\text {Landau }}^{\alpha}(x)+\mathscr{R}(x) \quad(\lambda=0),
$$

where $\Gamma_{\text {Landau }}^{\alpha}$ is a so-called Landau solution, and $\mathscr{R}(x)$ again a remainder that decays faster as $|x| \rightarrow \infty$ than $\Gamma_{\text {Landau }}^{\alpha}(x)$. A closed-form expression can be given for $\Gamma_{\text {Landau }}^{\alpha}$. 


\section{Introduction}

The expression depends on a parameter $\alpha \in \mathbb{R}^{3}$, which for the specific Landau solution in (1.18) is $\int_{\mathbb{R}^{3}} f(x) \mathrm{d} x$. The Landau solution was originally constructed by Landau in [40].

Very recently, as mentioned in Section 1.2, KAng, Miura, and Tsai [33] obtained in the case $\lambda=0$ an asymptotic expansion of a time-periodic solution to

$$
\begin{cases}\partial_{t} u-\Delta u-\lambda \partial_{1} u+\nabla \mathfrak{p}+u \cdot \nabla u=f & \text { in } \mathbb{R}^{3} \times(0, T), \\ \operatorname{div} u=0 & \text { in } \mathbb{R}^{3} \times(0, T), \\ \lim _{|x| \rightarrow \infty} u(x, t)=0 . & \end{cases}
$$

More precisely, it is shown in [33] that a sufficiently "small" strong solution satisfies

$$
u(x, t)=\Gamma_{\text {Landau }}^{\alpha}(x)+\mathscr{R}(x, t) \quad(\lambda=0),
$$

where $\Gamma_{\text {Landau }}^{\alpha}$ is the Landau solution determined by the parameter

$$
\alpha:=\frac{1}{\mathcal{T}} \int_{0}^{\mathcal{T}} \int_{\mathbb{R}^{3}} f(x, t) \mathrm{d} x \mathrm{~d} t .
$$

In other words, a time-periodic solution satisfies in the case $\lambda=0$ an asymptotic expansion with a similar asymptotic profile as in the corresponding steady-state case.

In Theorem 5.1.6 and Theorem 5.2.2 the asymptotic structure of a time-periodic strong solution is investigated in the case $\lambda \neq 0$. It is shown that a strong time-periodic solution to (1.19) satisfies

$$
u(x)=\Gamma_{\mathrm{O}}^{\lambda}(x) \cdot\left(\frac{1}{\mathcal{T}} \int_{0}^{\mathcal{T}} \int_{\mathbb{R}^{3}} f(x, t) \mathrm{d} x \mathrm{~d} t\right)+\mathscr{R}(x, t) \quad(\lambda \neq 0),
$$

where $\mathscr{R}(x, t)$ decays faster than $\Gamma_{\mathrm{O}}^{\lambda}$ as $|x| \rightarrow \infty$. Consequently, also in the case $\lambda \neq 0$ does the asymptotic profile of a time-periodic solution coincide with the asymptotic profile for the corresponding steady-state solution. Furthermore, the information obtained on the asymptotic structure is used to conclude that a strong solution has finite energy if and only if

$$
\frac{1}{\mathcal{T}} \int_{0}^{\mathcal{T}} \int_{\mathbb{R}^{3}} f(x, t) \mathrm{d} x \mathrm{~d} t=0
$$

The expansions (1.20) and (1.21) are not entirely surprising. A special case of the time-periodic Navier-Stokes problem has namely been studied extensively over the last years, and similar results have been obtained with respect to the asymptotic structure of the corresponding solutions. The special case in question is the Navier-Stokes system describing a fluid flow around a body that is rotating with a constant angular velocity. 
If this system is written in a frame of reference attached to the rotating body, the corresponding steady-state solutions are time-periodic when referred back to the inertial frame. Depending on whether the body is translating or not, one has $\lambda \neq 0$ or $\lambda=0$. In the case $\lambda=0$, an expansion similar to (1.20) was shown by FARWIG and HISHIDA in [8] with a decay estimate of the remainder term $\mathscr{R}$ given in the sense of summability. In [7] a pointwise decay estimate was established by FARwig, GALDI, and Kyed. The case $\lambda \neq 0$ was treated by KYED in [39], where an asymptotic expansion similar to (1.21) is established with a decay estimate of the remainder term $\mathscr{R}$ given in the sense of summability. As an application of Theorem 5.1.6, we shall establish in Section 5.3 a new proof of this result with a pointwise decay estimate of the remainder term. 



\section{Notation and preliminaries}

\subsection{Basic notation}

Points in domains $\mathbb{R}^{3} \times(0, T)$ are denoted by $(x, t)$ with $x \in \mathbb{R}^{3}$ and $t \in(0, T)$. We refer to $x$ as the spatial variable, and to $t$ as the time variable.

For any sufficiently regular function $u: \mathbb{R}^{3} \times(0, T) \rightarrow \mathbb{R}$ we put $\partial_{i} u:=\partial_{x_{i}} u$ for $i=1,2,3$. In addition, for any multiindex $\alpha \in \mathbb{N}_{0}^{3}$ we let

$$
\partial_{x}^{\alpha} u:=\mathrm{D}^{\alpha} u:=\sum_{j=1}^{3} \partial_{j}^{\alpha_{j}} u
$$

and put $|\alpha|:=\alpha_{1}+\alpha_{2}+\alpha_{3}$. Moreover, for $x \in \mathbb{R}^{3}$ we let $x^{\alpha}:=x_{1}^{\alpha_{1}} x_{2}^{\alpha_{2}} x_{3}^{\alpha_{3}}$.

We denote by $\Delta u$ the Laplacian of $u$ with respect to the spatial variable, that is,

$$
\Delta u: \mathbb{R}^{3} \times(0, T) \rightarrow \mathbb{R}, \quad \Delta u:=\sum_{i=1}^{3} \partial_{i}^{2} u .
$$

For a vector field $u: \mathbb{R}^{3} \times(0, T) \rightarrow \mathbb{R}^{3}$ we denote by $\nabla u$ the tensor field

$$
\nabla u: \mathbb{R}^{3} \times(0, T) \rightarrow \mathbb{R}^{3 \times 3}, \quad(\nabla u)_{i j}:=\partial_{j} u_{i} .
$$

In addition, we define the divergence of $u$ as

$$
\operatorname{div} u: \mathbb{R}^{3} \times(0, T) \rightarrow \mathbb{R}, \quad \operatorname{div} u:=\sum_{i=1}^{3} \partial_{i} u_{i} .
$$

For $u: \mathbb{R}^{3} \times(0, T) \rightarrow \mathbb{R}^{3}$ and $v: \mathbb{R}^{3} \times(0, T) \rightarrow \mathbb{R}^{3}$ we let $(u \cdot \nabla v): \mathbb{R}^{3} \times(0, T) \rightarrow \mathbb{R}^{3}$ denote the vector field

$$
(u \cdot \nabla v)_{i}:=\sum_{j=1}^{3} \partial_{j} v_{i} u_{j}
$$

For two vectors $a, b \in \mathbb{R}^{n}$ we let $a \otimes b \in \mathbb{R}^{n \times n}$ denote the tensor

$$
a \otimes b \in \mathbb{R}^{n \times n}, \quad(a \otimes b)_{i j}:=a_{i} b_{j} .
$$

For two second order tensors $A, B \in \mathbb{R}^{n \times n}$ we let $A: B:=\sum_{i, j=1}^{n} A_{i j} B_{i j}$. We denote by $I$ the identity $I \in \mathbb{R}^{n \times n}$. 
We use $\mathrm{B}_{R}:=\left\{x \in \mathbb{R}^{n}|| x \mid<R\right\}$ to denote balls in $\mathbb{R}^{n}$. Furthermore, for $R_{1}<R_{2}$ we let $\mathrm{B}_{R_{2}, R_{1}}:=\left\{x \in \mathbb{R}^{n}\left|R_{1}<\right| x \mid<R_{2}\right\}$. In addition, we put $\mathrm{B}^{R}:=\mathbb{R}^{n} \backslash \mathrm{B}_{R}$.

For a sequence $\left\{x_{n}\right\}_{n=1}^{\infty}$ in a normed vector space $X$, we use $x_{n} \rightarrow x$ to denote strong convergence, and $x_{n} \rightarrow x$ to denote weak convergence.

If $X$ is a vector space and $X^{\prime}$ its dual, we use the notation $\langle\varphi, x\rangle$ to denote the pairing of a functional $\varphi \in X^{\prime}$ and an element $x \in X$.

We will make use of the Landau symbols, that is, the Big-O and Small-o notation. Recall that $f(x)=O(g(x))$ iff $|f(x)| \leq C|g(x)|$ as $|x| \rightarrow \infty$, and $f(x)=o(g(x))$ iff $|f(x)| /|g(x)| \rightarrow 0$ as $|x| \rightarrow \infty$.

For $X$ a topological vector space and $A, B \subset X$, we write $X=A \oplus B$ iff $A$ and $B$ are closed subspaces of $X$ with $A \cap B=\{0\}$ and $X=A+B$.

Constants in capital letters in the proofs and theorems are global, while constants in small letters are local to the proof in which they appear.

\subsection{Topological groups and the Fourier transform}

We use $G$ to denote a locally compact abelian group. We let $\int_{G} \mathrm{~d} g$ denote the Haarintegral corresponding the Haar measure $\mathrm{d} g$ on the group. Recall that the Haar measure is uniquely defined up-to multiplication by a constant. For a compact group we always consider the normalized Haar measure. In particular, for a torus group $\mathbb{T}:=\mathbb{R} / \mathcal{T} \mathbb{Z}$, $\mathcal{T}>0$ some constant, we always have $\int_{\mathbb{T}} 1 \mathrm{~d} t=1$.

For a locally compact abelian group $G$, we denote by $\widehat{G}$ the corresponding dual group consisting of the continuous multiplicative characters $\chi: G \rightarrow \mathbb{C}$. The dual group is equipped with the compact-open topology. It is well-known that this topology turns $\widehat{G}$ into a locally compact abelian group.

For a complex Hilbert space $\mathscr{H}$, the Fourier transform is defined by

$$
\mathcal{F}: L^{1}(G ; \mathscr{H}) \rightarrow C(\widehat{G} ; \mathscr{H}), \quad \mathcal{F}(f)(\chi):=\widehat{f}(\chi):=\int_{G} f(\chi) \overline{\chi(g)} \mathrm{d} g,
$$

where the integral is understood as the Bochner integral in $\mathscr{H}$ with respect to the Haar measure on $G$.

The inverse Fourier transform is formally defined by

$$
\mathcal{F}^{-1}: L^{1}(\widehat{G} ; \mathscr{H}) \rightarrow C(G ; \mathscr{H}), \quad \mathcal{F}^{-1}(f)(g):=f^{\vee}(g):=\int_{\widehat{G}} f(\chi) \chi(g) \mathrm{d} \chi .
$$

By classical theory, the Fourier transform, viewed as a mapping

$$
\mathcal{F}: L^{1}(G ; \mathscr{H}) \cap L^{2}(G ; \mathscr{H}) \rightarrow C(\widehat{G} ; \mathscr{H}),
$$

extends uniquely to a unitary transformation

$$
\mathcal{F}: L^{2}(G ; \mathscr{H}) \rightarrow L^{2}(\widehat{G} ; \mathscr{H}) .
$$

The inverse of this mapping coincides on $L^{1}(\widehat{G})$ with $\mathcal{F}^{-1}$. 
Example 2.2.1. Consider $G:=\mathbb{T}:=\mathbb{R} / \mathcal{T} \mathbb{Z}$. Each $k \in \mathbb{Z}$ can then be identified with the character $t \rightarrow \mathrm{e}^{i \frac{2 \pi}{T} k t}$. It is easy to verify that each character on $G$ is of this type. We thus have $\widehat{G}=\mathbb{Z}$. The compact-open topology of $\widehat{G}$ coincides with the discrete topology on $\mathbb{Z}$. Consequently, we recover from the Fourier transform (2.1) in this case the theory of Fourier series.

Example 2.2.2. Consider $G:=\mathbb{R}^{3}$. Each $\xi \in \mathbb{R}^{3}$ can then be identified with the character $x \rightarrow \mathrm{e}^{i \xi \cdot x}$. One may verify that each character on $G$ is of this type. We thus have $\widehat{G}=\mathbb{R}^{3}$. The compact-open topology of $\widehat{G}$ coincides with the Euclidean topology on $\mathbb{R}^{3}$. We therefore recover from the Fourier transform (2.1) in this case the theory of the classical Fourier transform.

\subsection{Function spaces}

\subsubsection{Sobolev spaces}

For any domain $\Omega \subset \mathbb{R}^{n}$ and $k \in \mathbb{N}_{0}$ we denote by $C^{k}(\Omega)$ the space of all functions $u: \Omega \rightarrow \mathbb{R}$ for which $\partial^{\alpha} u$ exists and is continuous in $\Omega$ for all $\alpha \in \mathbb{N}_{0}$ with $|\alpha| \leq k$. We put $C^{\infty}(\Omega):=\bigcap_{k=0}^{\infty} C^{k}(\Omega)$. By $C_{0}^{k}(\Omega)$ we denote the space of all functions in $C^{k}(\Omega)$ with compact support.

By $L^{q}(\Omega)$ we denote for $q \in[1, \infty)$ the usual Lebesgue space with norm

$$
\|f\|_{q}:=\left(\int_{\Omega}|f(x)|^{q} \mathrm{~d} x\right)^{1 / q} .
$$

For $m \in \mathbb{N}_{0}$ we denote by $W^{m, q}(\Omega)$ the inhomogeneous Sobolev space

$$
W^{m, q}(\Omega):=\left\{u \in L_{l o c}^{1}(\Omega) \mid \forall \alpha \in \mathbb{N}_{0}^{n} \text { with }|\alpha| \leq m: \partial^{\alpha} u \in L^{q}(\Omega)\right\}
$$

equipped with the norm

$$
\|u\|_{m, q}:=\left(\sum_{|\alpha| \leq m} \int_{\Omega}\left|\partial^{\alpha} u(x)\right|^{q} \mathrm{~d} x\right)^{\frac{1}{q}} .
$$

By $D^{m, q}(\Omega)$ we denote the homogeneous Sobolev space

$$
D^{m, q}(\Omega):=\left\{u \in L_{l o c}^{1}(\Omega) \mid \forall \alpha \in \mathbb{N}_{0}^{3} \text { with }|\alpha|=m: \partial^{\alpha} u \in L^{q}(\Omega)\right\}
$$

equipped with the semi-norm $D^{m, q}(\Omega)$

$$
|u|_{m, q}:=\left(\sum_{|\alpha|=m} \int_{\Omega}\left|\partial^{\alpha} u(x)\right|^{q} \mathrm{~d} x\right)^{\frac{1}{q}} .
$$

We observe that $|\cdot|_{m, q}$ defines a norm on $C_{0}^{\infty}(\Omega)$, and let

$$
D_{0}^{m, q}(\Omega):={\overline{C_{0}^{\infty}(\Omega)}}^{|\cdot|_{m, q}}
$$


denote the closure with respect to this norm. We use the notation $D_{0}^{-m, q}(\Omega)$ to denote the dual space of $D_{0}^{m, q^{\prime}}(\Omega)$, where $q^{\prime}:=\frac{q}{q-1}$ is the Hölder conjugate of $q$. We denote by $|\cdot|_{-m, q}$ the norm of $D_{0}^{-m, q}(\Omega)$.

We use $C_{0, \sigma}^{\infty}(\Omega)$ to denote the space of all smooth solenoidal vector fields, that is,

$$
C_{0, \sigma}^{\infty}(\Omega):=\left\{u \in C_{0}^{\infty}(\Omega)^{n} \mid \operatorname{div} u=0\right\} .
$$

We further introduce the spaces

$$
\begin{aligned}
& L_{\sigma}^{q}(\Omega):={\overline{C_{0, \sigma}^{\infty}(\Omega)}}^{\|\cdot\|_{q}}, \\
& D_{0, \sigma}^{m, q}(\Omega):=\left.{\overline{C_{0, \sigma}^{\infty}(\Omega)}}^{\mid} \cdot\right|_{m, q}, \\
& W_{0, \sigma}^{m, q}(\Omega):={\overline{C_{0, \sigma}^{\infty}(\Omega)}}^{\|\cdot\|_{m, q}}
\end{aligned}
$$

of solenoidal vector fields.

\subsubsection{Sobolev embedding}

For classical versions of Sobolev's embedding theorem the reader is referred to literature such as [1]. Below, we recall some embedding theorems that are not always found in standard literature.

Lemma 2.3.1. Let $q \in[1,3), r \in[q, 3 q /(3-q)]$, and

$$
\lambda:=\frac{3(r-q)}{r q} .
$$

Then

$$
\forall u \in C_{0}^{\infty}\left(\mathbb{R}^{3}\right): \quad\|u\|_{r} \leq C_{1}\|u\|_{q}^{1-\lambda}\|\nabla u\|_{q}^{\lambda},
$$

with $C_{1}=C_{1}(\lambda, q, r)$.

Proof. See for example [17, Lemma II.2.2].

As a direct consequence of Lemma 2.3.1 it follows that

$$
\forall u \in D_{0}^{1,2}\left(\mathbb{R}^{3}\right):\|u\|_{L^{6}\left(\mathbb{R}^{3}\right)} \leq C_{2}\|\nabla u\|_{L^{2}\left(\mathbb{R}^{3}\right)} .
$$

We shall make use of the following embedding property of the homogeneous Sobolev spaces:

Lemma 2.3.2. Let $q \in(1, \infty)$ and $r \in(3, \infty)$. Then

$$
\forall u \in D^{1, r}\left(\mathbb{R}^{3}\right) \cap L^{q}\left(\mathbb{R}^{3}\right): \quad\|u\|_{\infty} \leq C_{3}\left(|u|_{1, r}+\|u\|_{q}\right)
$$

with $C_{3}=C_{3}(q, r)$. 
Proof. See [17, Remark II.7.2].

The following Hardy-type inequality shall later play an important role.

Lemma 2.3.3. Let $q \in(1,3), r \in(1, \infty)$, and $v \in D^{1, q}\left(\mathbb{R}^{3}\right) \cap L^{r}\left(\mathbb{R}^{3}\right)$. If $\left|x_{0}\right|=\alpha R$ for some $\alpha \geq \alpha_{0}>1$, then

$$
\left(\int_{\mathrm{B}^{R}} \frac{|v(x)|^{q}}{\left|x-x_{0}\right|^{q}} \mathrm{~d} x\right)^{\frac{1}{q}} \leq C_{4}\left(\int_{\mathrm{B}^{R}}|\nabla v(x)|^{q} \mathrm{~d} x\right)^{\frac{1}{q}}
$$

with $C_{4}=C_{4}\left(q, \alpha_{0}\right)$.

Proof. See [18, Theorem II.5.1].

\subsubsection{Helmholtz-Weyl decomposition}

For $q \in[1, \infty)$ we put

$$
\mathscr{G}^{q}(\Omega):=\left\{w \in L^{q}(\Omega)^{3} \mid w=\nabla p \text { for some } p \in W_{l o c}^{1, q}(\Omega)\right\} .
$$

The following lemma states the validity of the so-called Helmholtz-Weyl decomposition of the space $L^{q}\left(\mathbb{R}^{3}\right)^{3}$.

Lemma 2.3.4. For any $q \in(1, \infty)$ the decomposition

$$
L^{q}\left(\mathbb{R}^{3}\right)^{3}=L_{\sigma}^{q}\left(\mathbb{R}^{3}\right) \oplus \mathscr{G}^{q}\left(\mathbb{R}^{3}\right)
$$

is valid. The corresponding projection

$$
\mathcal{P}_{H}: L^{q}\left(\mathbb{R}^{3}\right)^{3} \rightarrow L_{\sigma}^{q}\left(\mathbb{R}^{3}\right)
$$

is called the Helmholtz projection.

Proof. See for example [17, Theorem III.1.2].

The Helmholtz projection in the whole-space can be expressed explicitly as a Fourier multiplier. More specifically:

Lemma 2.3.5. For $f \in C_{0}^{\infty}\left(\mathbb{R}^{3}\right)^{3}$ the Helmholtz projection is given by

$$
\mathcal{P}_{H} f=\mathcal{F}^{-1}\left[\left(I-\frac{\xi \otimes \xi}{|\xi|^{2}}\right) \widehat{f}\right] .
$$

Proof. According to (2.5) we have $f=f_{\sigma}+\nabla p$ with $f \in L_{\sigma}^{q}\left(\mathbb{R}^{3}\right)$ and $p \in W_{l o c}^{1, q}\left(\mathbb{R}^{3}\right)$. It follows that $\operatorname{div} f=\Delta p$ in the sense of distributions. Applying the Fourier transform to this identify yields $(2.6)$. 


\subsubsection{Function spaces involving time}

If $X$ is a Banach space and $S$ a measure space with positive measure $\mu$, we let $L^{q}(S ; X)$, $q \in[1, \infty)$, denote the usual Bochner-Lebesgue space of Bochner measurable functions $u: S \rightarrow X$ for which

$$
\|u\|_{L^{q}(S ; X)}:=\left(\int_{S}\|u(t)\|_{X}^{q} \mathrm{~d} \mu\right)^{1 / q}<\infty .
$$

Similarly, $L^{\infty}(S ; X)$ is defined as the space of all Bochner-measurable functions $u: S \rightarrow$ $X$ for which

$$
\|u\|_{L^{\infty}(S ; X)}:=\underset{t \in S}{\operatorname{ess} \sup }\|u(t)\|_{X}<\infty .
$$

As is standard in the theory of classical $L^{q}$-space, we silently identify $L^{q}(S ; X)$ with the quotient space of equivalence classes of functions that differ only on a set of measure zero. Consequently, $\left(L^{q}(S ; X),\|\cdot\|_{L^{q}(S ; X)}\right)$ are Banach spaces, but elements of $L^{q}(S ; X)$ do not have pointwise definitions. The latter is important to keep in mind since it prevents us from introducing directly the notion of periodicity of elements in $L^{q}(S ; X)$. If for instance $S$ is the interval $[0, T]$, the requirement that $u(0)=u(T)$ does not make sense for elements $u \in L^{q}(S ; X)$.

The Bochner spaces will be used to introduce function spaces involving time. More precisely, we either take $S$ to be a time interval $[0, T]$ equipped with the Lebesgue measure, or the torus group $\mathbb{T}:=\mathbb{R} / \mathcal{T} \mathbb{Z}$ equipped with the normalized Haar measure. In the latter case $\mathcal{T}>0$ will be some positive constant used to denote the period in a setting of time-periodic functions.

\subsection{Multiplier theory}

We shall make use of the following multiplier theorem of Marcinkiewicz type:

Theorem 2.4.1. Let $m: \mathbb{R}^{n} \rightarrow \mathbb{C}$ be a bounded function with $m \in C^{n}\left(\mathbb{R}^{n}\right)$. Assume there is a constant $A$ such that

$$
\sup _{\varepsilon \in\{0,1\}^{n}} \sup _{\xi \in \mathbb{R}^{n}}\left|\xi_{1}^{\varepsilon_{1}} \ldots \xi_{n}^{\varepsilon_{n}} \partial_{\xi_{1}}^{\varepsilon_{1}} \ldots \partial_{\xi_{n}}^{\varepsilon_{n}} m(\xi)\right| \leq A .
$$

Then for any $q \in(1, \infty)$ there is a constant $C$ such that

$$
\forall f \in L^{2}\left(\mathbb{R}^{n}\right) \cap L^{q}\left(\mathbb{R}^{n}\right):\left\|\mathcal{F}^{-1}[m \cdot \widehat{f}]\right\|_{q} \leq C A\|f\|_{q},
$$

with $C=C(q)$.

Proof. The result is originally due to MARCinkiewicz [43], who established the theorem in the context of Fourier series. A proof of the version above can be found in, for example, [28, Corollary 5.2.5] and [54, Chapter IV, §6].

Remark 2.4.2. Versions of the Marcinkiewicz multiplier theorem are available with weaker assumptions on the regularity of $m$. The theorem as stated above, however, suffices for the purposes we shall be using it for in the following. 


\section{Linear theory}

In this chapter a comprehensive investigation of the linearized time-periodic NavierStokes problem is carried out. More specifically, the linear problem

$$
\begin{cases}\partial_{t} u-\Delta u-\lambda \partial_{1} u+\nabla \mathfrak{p}=f & \text { in } \mathbb{R}^{3} \times \mathbb{R} \\ \operatorname{div} u=0 & \text { in } \mathbb{R}^{3} \times \mathbb{R} \\ \lim _{|x| \rightarrow \infty} u(x, t)=0, & \\ u(\cdot, t+\mathcal{T})=u(\cdot, t) & \end{cases}
$$

with time-periodic data

$$
f(\cdot, t+\mathcal{T})=f(\cdot, t)
$$

is considered. The problem is analyzed in an $L^{q}$-setting. More specifically, the resolvability of the equations for a right-hand side $f \in L^{q}\left(\mathbb{R}^{3} \times(0, \mathcal{T})\right)^{3}$ is investigated. Observe that any element $f \in L^{q}\left(\mathbb{R}^{3} \times(0, \mathcal{T})\right)^{3}$ can be trivially extended to a time-periodic function satisfying (3.2).

The main purpose of the chapter is to identify a Banach space $\mathrm{X}^{q}$ such that for any $f \in L^{q}\left(\mathbb{R}^{3} \times(0, \mathcal{T})\right)^{3}$ there is a unique solution $(u, \mathfrak{p})$ in $\mathrm{X}^{q}$ with

$$
\|(u, \mathfrak{p})\|_{\mathrm{X}^{q}} \leq C\|f\|_{q} .
$$

In other words, we shall seek to establish a functional analytic setting in which (3.1) can be written on an operator form

$$
A(u, \mathfrak{p})=f
$$

such that

$$
A: \mathrm{X}^{q} \rightarrow L^{q}\left(\mathbb{R}^{3} \times(0, \mathcal{T})\right)^{3}
$$

is a homeomorphism. We refer to such a function space as a maximal regularity framework in the $L^{q}$-setting for the linearized time-periodic Navier-Stokes system.

In order to identify the space $\mathrm{X}^{q}$, we shall employ the theory of Fourier multipliers. This may not seem surprising, as similar results for both the corresponding steady-state and initial-value problem are traditionally established using Fourier multipliers. However, it is not directly clear how to employ the Fourier transform on the space-time domain $\mathbb{R}^{3} \times(0, \mathcal{T})$. The main idea behind the approach presented in the following is to identify $\mathbb{R}^{3} \times(0, \mathcal{T})$ with the group $G:=\mathbb{R}^{3} \times \mathbb{R} / \mathcal{T} \mathbb{Z}$. Equipped with the canonical 


\section{Linear theory}

topology, $G$ is a locally compact abelian group. As such, there is a naturally defined Fourier transform $\mathcal{F}_{G}$ associated to $G$. Moreover, as $G$ inherits a differentiable structure from $\mathbb{R}^{3} \times \mathbb{R}$ in a canonical way, we may view (3.1) as a system of differential equations on $G$. Employing the Fourier transform $\mathcal{F}_{G}$, we then obtain a representation of the solution in terms of a Fourier multiplier defined on the dual group $\widehat{G}$. Based on this representation, the space $\mathrm{X}^{q}$ and corresponding a priori estimate (3.3) will be established. Since multiplier theorems like the theorems of Mihlin, Lizorkin or Marcinkiewicz are not available in the general case of group multipliers, we shall employ a so-called transference principle. More specifically, we shall use a theorem, which in its original form is due to DE LEEUW [4], that enables us to study the properties of a multiplier defined on $\widehat{G}$ in terms of a corresponding multiplier defined on $\mathbb{R}^{4}$. As we shall see, the time-averaging decomposition introduced in Section 1.5 will play an important role in the "transference" of the multiplier between $\widehat{G}$ and $\mathbb{R}^{4}$.

The chapter is organized as follows. In Section 3.1 the abstract approach based on topological groups is motivated. In Section 3.2 we reformulate (3.1) as a system of differential equations on the group $G$. In Section 3.3 we employ the corresponding Fourier transform on the group to establish a representation formula for the solution $u$ in terms of a Fourier multiplier. In Section 3.4 the transference principle for multipliers on groups is introduced. The time-averaging projection in the context of $G$-defined vector fields is defined in Section 3.5. Finally, in Section 3.6 we identify $\mathrm{X}^{q}$ and show the corresponding a priori estimate (3.3).

\subsection{Fourier series expansion in time}

The introduction of the group $G:=\mathbb{R}^{3} \times \mathbb{R} / \mathcal{T} \mathbb{Z}$ is based on the simple idea of expanding both the time-periodic data $f$ and time-periodic solution $(u, \mathfrak{p})$ to $(3.1)$ in a Fourier series with respect to time, and subsequently applying the Fourier transform in $\mathbb{R}^{3}$ with respect the spatial variable. In this way, a representation formula for the solution in terms of the data can be derived. We shall here briefly discuss the challenges of deriving from this formula the a priori estimate (3.3). In this context, the benefits of employing an abstract approach based on topological groups becomes clear.

If the data $f: \mathbb{R}^{3} \times \mathbb{R} \rightarrow \mathbb{R}^{3}$ is time periodic with period $\mathcal{T}$ and sufficiently regular, we can expand $t \rightarrow f(\cdot, t)$ in a Fourier series with respect to the Fourier basis $\left\{\mathrm{e}^{i \frac{2 \pi}{\mathcal{T}} k t} \mid k \in \mathbb{Z}\right\}$. We then have

$$
f(x, t)=\sum_{k \in \mathbb{Z}} f_{k}(x) \mathrm{e}^{i \frac{2 \pi}{T} k t}
$$

with

$$
f_{k}: \mathbb{R}^{3} \rightarrow \mathbb{R}^{3}, \quad f_{k}(x):=\frac{1}{\mathcal{T}} \int_{0}^{\mathcal{T}} f(x, t) \mathrm{e}^{-i \frac{2 \pi}{\mathcal{T}} k t} \mathrm{~d} t
$$


Similarly, we can expand a time-periodic solution $(u, \mathfrak{p})$ in a Fourier series

$$
u(x, t)=\sum_{k \in \mathbb{Z}} u_{k}(x) \mathrm{e}^{i \frac{2 \pi}{\mathcal{T}} k t}
$$

with

$$
u_{k}: \mathbb{R}^{3} \rightarrow \mathbb{R}^{3}, \quad u_{k}(x):=\frac{1}{\mathcal{T}} \int_{0}^{\mathcal{T}} u(x, t) \mathrm{e}^{-i \frac{2 \pi}{\mathcal{T}} k t} \mathrm{~d} t
$$

and

$$
\mathfrak{p}(x, t)=\sum_{k \in \mathbb{Z}} \mathfrak{p}_{k}(x) \mathrm{e}^{i \frac{2 \pi}{\mathcal{T}} k t}, \quad \mathfrak{p}_{k}(x):=\frac{1}{\mathcal{T}} \int_{0}^{\mathcal{T}} \mathfrak{p}(x, t) \mathrm{e}^{-i \frac{2 \pi}{\mathcal{T}} k t} \mathrm{~d} t .
$$

Inserting the Fourier series corresponding to $f, u$, and $\mathfrak{p}$ in (3.1), we obtain for each $k \in \mathbb{Z}$ the system

$$
\begin{cases}i \frac{2 \pi}{\mathcal{T}} k u_{k}-\Delta u_{k}-\lambda \partial_{1} u_{k}+\nabla \mathfrak{p}_{k}=f_{k} & \text { in } \mathbb{R}^{3} \\ \operatorname{div} u_{k}=0 & \text { in } \mathbb{R}^{3} \\ \lim _{|x| \rightarrow \infty} u_{k}(x)=0 . & \end{cases}
$$

Thus, each Fourier coefficient $\left(u_{k}, \mathfrak{p}_{k}\right)$ of the solution satisfies a resolvent-like Oseen $(\lambda \neq 0)$ or Stokes $(\lambda=0)$ system. If we in (3.5) employ the classical Fourier transform, we obtain the representation formula

$$
u_{k}(x)=\mathcal{F}_{\mathbb{R}^{3}}^{-1}\left[\frac{1}{|\xi|^{2}+i\left(\frac{2 \pi}{\mathcal{T}} k-\lambda \xi_{1}\right)}\left(I-\frac{\xi \otimes \xi}{|\xi|^{2}}\right) \widehat{f}_{k}\right]
$$

where we recognize, recall Lemma 2.3.5, the symbol $I-\frac{\xi \otimes \xi}{|\xi|^{2}}$ of the Helmholtz decomposition.

Since (3.6) is a representation of $u_{k}$ in terms of a classical Fourier multiplier in $\mathbb{R}^{3}$, it is possible at this point to employ the classical multiplier theorems of either Mihlin, Lizorkin, or Marcinkiewicz to establish an estimate of $u_{k}$ in terms of $f_{k}$. This would lead to an estimate of the type

$$
\left\|\mathrm{D}^{\alpha} u_{k}\right\|_{p} \leq C\left\|f_{k}\right\|_{q}
$$

with a constant $C$ depending on $p, q, \alpha$, and $k$. The challenge is then to use the estimate (3.7) of the Fourier coefficients to establish an estimate of $u$ in terms of $f$. This will be possible if $C=C(k)$ decays sufficiently fast as $k \rightarrow \infty$. It seems the best estimate one can obtain in this respect is

$$
\left\|u_{k}\right\|_{q} \leq \frac{c}{|k|}\left\|f_{k}\right\|_{q}
$$




\section{Linear theory}

Using for example the Hausdorff-Young inequality, it is in fact possible to derive from (3.8) some estimate of $u$. However, it turns out that this bound is not optimal. For the second order derivatives of $u_{k}$, that is, the case $|\alpha|=2$, it seems (3.7) can only be obtained with a constant $C$ that does not decay at all as $k \rightarrow \infty$. In this case, it is not possible to derive from (3.7) any meaningful estimate on $\mathrm{D}^{\alpha} u$. We thus observe that classical multiplier theory applied to the Fourier coefficients $u_{k}$ does not achieve the goal of identifying the maximal regularity space $\mathrm{X}^{q}$. In other words, too much information is lost by estimating each Fourier coefficient $u_{k}$ separately for each $k \in \mathbb{Z}$. This insight is main reason for introducing the group $G:=\mathbb{R}^{3} \times \mathbb{R} / \mathcal{T} \mathbb{Z}$. The Fourier transform $\mathcal{F}_{G}$ corresponding to $G$ namely acts both in the time and space variable at the same time. If we are therefore able to employ $\mathcal{F}_{G}$ in (3.1), we can anticipate a representation of $u$ itself in terms of a $G$-multiplier. Although it is not clear how to obtain from an abstract groupmultiplier representation an estimate on $u$, if we do succeed, we can expect a sharper estimate than can be obtained by estimating each Fourier coefficient of $u$ separately.

Before moving on to the development of a multiplier approach based on the group $G$, we briefly discuss an important property of the decomposition introduced in Section 1.5 of a time-periodic function into a steady-state and time-periodic part. In connection with the Fourier series introduced above, it is easy to see that decomposition from Section 1.5 simply splits the zeroth order Fourier coefficient from the rest of the series. For example, recalling (3.4) and the definition (1.12), we have

$$
u_{s}=u_{0}, \quad u_{p}=\sum_{k \in \mathbb{Z} \backslash\{0\}} u_{k}(x) \mathrm{e}^{i \frac{2 \pi}{\mathcal{T}} k t} .
$$

Going back to (3.5), we see that $u_{0}$ satisfies the classical steady-state Oseen $(\lambda \neq 0)$ or Stokes $(\lambda \neq 0)$ problem. In terms of the multiplier representation (3.6), this means that the multiplier

$$
m_{k}(\xi):=\frac{1}{|\xi|^{2}+i\left(k-\lambda \xi_{1}\right)}
$$

in the case $k=0$ equals the characteristic multiplier of the Oseen $(\lambda \neq 0)$ or Stokes $(\lambda \neq 0)$ problem. Note that the multiplier $m_{0}$ has a singularity at $\xi=0$. In the case $k \neq 0$, however, the multiplier $m_{k}$ does not have any singularities. This observation is crucial for understanding the merits of the decomposition. It namely means that $u_{k}$, $k \neq 0$, in general decays faster at infinity than $u_{0}$, or, in other words, the generic estimate one can obtain of $u_{k}, k \neq 0$, in terms of the data is better than what can be obtained for $u_{0}$. This motivates the idea of decomposing the space $\mathrm{X}^{q}$ into a direct sum of two spaces. One space to establish the maximal regularity of steady-state part $u_{s}$ of the solution, and another space to do the same for the time-periodic part $u_{p}$. As we shall see in the following, this idea is crucial for the identification of the space $\mathrm{X}^{q}$. 


\subsection{Reformulation on a group}

Here and in the rest of this chapter, we let $G$ denote the group

$$
G:=\mathbb{R}^{3} \times \mathbb{R} / \mathcal{T} \mathbb{Z}
$$

with addition as group operation. In order to study (3.1) in a setting of functions defined on $G$, we must first introduce a topology and an appropriate differentiable structure on $G$. It is then possible to define Lebesgue and Sobolev spaces on $G$. We will study (3.1) in such spaces.

\subsubsection{Differentiable structure}

The topology and differentiable structure is inherited from $\mathbb{R}^{3} \times \mathbb{R}$. More precisely, we equip $G$ with the quotient topology induced by the canonical quotient mapping

$$
\pi: \mathbb{R}^{3} \times \mathbb{R} \rightarrow \mathbb{R}^{3} \times \mathbb{R} / \mathcal{T} \mathbb{Z}, \quad \pi(x, t):=(x,[t]) .
$$

Equipped with the quotient topology, $G$ becomes a locally compact abelian group. Moreover, we define by

$$
C^{\infty}(G):=\left\{u: G \rightarrow \mathbb{R} \mid \exists U \in C^{\infty}\left(\mathbb{R}^{3} \times \mathbb{R}\right): U=u \circ \pi\right\}
$$

the space of smooth functions on $G$, and for $u \in C^{\infty}(G)$ the derivatives

$$
\forall(\alpha, \beta) \in \mathbb{N}_{0}^{3} \times \mathbb{N}_{0}: \quad \partial_{t}^{\beta} \partial_{x}^{\alpha} u:=\partial_{t}^{\beta} \partial_{x}^{\alpha} U_{\mid \mathbb{R}^{3} \times[0, \mathcal{T})},
$$

where $G$ is identified with $\mathbb{R}^{3} \times[0, \mathcal{T})$ via the canonical bijection. It is easy to verify for $u \in C^{\infty}(G)$ that also $\partial_{t}^{\beta} \partial_{x}^{\alpha} u \in C^{\infty}(G)$. We further introduce the subspace

$$
C_{0}^{\infty}(G):=\left\{u \in C^{\infty}(G) \mid \operatorname{supp} u \text { is compact }\right\}
$$

of compactly supported smooth functions. Clearly, $C_{0}^{\infty}(G) \subset C_{0}(G)$, where $C_{0}(G)$ denotes the space of continuous functions of compact support.

With a differentiable structure defined on $G$ via (3.11), we can introduce the space of tempered distributions on $G$. For this purpose, we first recall the Schwartz-Bruhat space of generalized Schwartz functions; see for example [3]. More precisely, we define for $u \in C^{\infty}(G)$ the semi-norms

$$
\forall(\alpha, \beta) \in \mathbb{N}_{0}^{3} \times \mathbb{N}_{0} \forall \gamma \in \mathbb{N}_{0}^{3}: \quad \rho_{\gamma, \alpha, \beta}(u):=\sup _{(x, t) \in G}\left|x^{\gamma} \partial_{t}^{\beta} \partial_{x}^{\alpha} u(x, t)\right|,
$$

and put

$$
\mathscr{S}(G):=\left\{u \in C^{\infty}(G) \mid \forall(\alpha, \beta) \in \mathbb{N}_{0}^{3} \times \mathbb{N}_{0} \forall \gamma \in \mathbb{N}_{0}^{3}: \rho_{\gamma, \alpha, \beta}(u)<\infty\right\} .
$$

Clearly, $\mathscr{S}(G)$ is a vector space, and $\rho_{\gamma, \alpha, \beta}$ a semi-norm on $\mathscr{S}(G)$. We endow $\mathscr{S}(G)$ with the semi-norm topology induced by the family $\left\{\rho_{\gamma, \alpha, \beta} \mid(\alpha, \beta) \in \mathbb{N}_{0}^{3} \times \mathbb{N}_{0}, \gamma \in \mathbb{N}_{0}^{3}\right\}$ of 


\section{Linear theory}

semi-norms. The topological dual space $\mathscr{S}^{\prime}(G)$ of $\mathscr{S}(G)$ is then well-defined. We equip $\mathscr{S}^{\prime}(G)$ with the weak* topology, and refer to it as the space of tempered distributions on $G$.

It is clear that $\mathscr{S}(G)$ is closed under multiplication by finite polynomials in the spatial variable, that is, for any finite polynomial of type $p(x):=\sum_{\alpha \in \mathbb{N}_{0}^{3}} a_{\alpha} x^{\alpha}$ and $\psi \in \mathscr{S}(G)$ we have $p \cdot \psi \in \mathscr{S}(G)$. By duality, $\mathscr{S}^{\prime}(G)$ also remains closed under multiplication by such polynomials. Similarly, both $\mathscr{S}(G)$ and $\mathscr{S}^{\prime}(G)$ remain closed under multiplication by smooth functions that have at most polynomial growth with respect to the spatial variables. In particular, $\mathscr{S}(G)$ and $\mathscr{S}^{\prime}(G)$ remain closed under multiplication by elements $\psi \in \mathscr{S}(G)$.

For a tempered distribution $u \in \mathscr{S}^{\prime}(G)$, distributional derivatives $\partial_{t}^{\beta} \partial_{x}^{\alpha} u \in \mathscr{S}^{\prime}(G)$ are defined in the usual manner:

$$
\forall \psi \in \mathscr{S}(G):\left\langle\partial_{t}^{\beta} \partial_{x}^{\alpha} u, \psi\right\rangle:=\left\langle u,(-1)^{|(\alpha, \beta)|} \partial_{t}^{\beta} \partial_{x}^{\alpha} \psi\right\rangle .
$$

It is easy to verify that $\partial_{t}^{\beta} \partial_{x}^{\alpha} u$ is well-defined as an element in $\mathscr{S}^{\prime}(G)$.

For differentiable functions or tempered distributions on $G$, we keep the convention that differential operators act only in the spatial variable unless otherwise indicated. More specifically, for $u: G \rightarrow \mathbb{R}^{n}$ we let $\Delta u(x, t):=\Delta_{x} u(x, t), \operatorname{div} u(x, t):=\operatorname{div}_{x} u(x, t)$, $\nabla u:=\nabla_{x} u$, etc.

The function spaces $C^{\infty}(G), C_{0}^{\infty}(G), \mathscr{S}(G)$, and $\mathscr{S}^{\prime}(G)$ were defined above as real vector spaces of real functions. Clearly, we can define them analogously as complex vector spaces of complex functions. In following, we shall employ the spaces in both roles. If confusion can arise, we shall explicitly indicate if a given function space is real or complex.

\subsubsection{Function spaces}

The Haar measure $\mathrm{d} g$ on $G$ is given by the product of the Lebesgue measure on $\mathbb{R}^{3}$ and the Lebesgue measure on $[0, \mathcal{T})$. The Haar measure is unique up-to a normalization factor, which we choose such that

$$
\forall u \in C_{0}(G): \quad \int_{G} u(g) \mathrm{d} g=\frac{1}{\mathcal{T}} \int_{0}^{\mathcal{T}} \int_{\mathbb{R}^{3}} u(x, t) \mathrm{d} x \mathrm{~d} t .
$$

We let $L^{q}(G)$ denote the usual Lebesgue space with respect to the Haar measure $\mathrm{d} g$, that is,

$$
\begin{aligned}
& L^{q}(G):=\left\{u \in L_{l o c}^{1}(G) \mid\|u\|_{q}<\infty\right\} \\
& \|u\|_{q}:=\left(\frac{1}{\mathcal{T}} \int_{0} \int_{\mathbb{R}^{3}}|u(x, t)|^{q} \mathrm{~d} x \mathrm{~d} t\right)^{1 / q}
\end{aligned}
$$


It is easy to show by a standard mollifier argument that $C_{0}(G)$ is a dense subset of $L^{q}(G)$. In fact, by choosing a smooth mollifier, one obtains that also $C_{0}^{\infty}(G)$ is a dense subset of $L^{q}(G)$; see also the proof of Lemma 3.2.1 below.

Sobolev spaces are defined for $k \in \mathbb{N}_{0}$ and $q \in[1, \infty)$ by

$$
\begin{aligned}
& W^{k, q}(G):=\left\{u \in L^{q}(G) \mid\|u\|_{k, q}<\infty\right\}, \\
& \|u\|_{k, q}:=\left(\sum_{|(\alpha, \beta)| \leq k}\left\|\partial_{t}^{\beta} \partial_{x}^{\alpha} u\right\|_{q}^{q}\right)^{1 / q} .
\end{aligned}
$$

Clearly, any $u \in L^{q}(G)$ belongs to $\mathscr{S}^{\prime}(G)$, so $\partial_{t}^{\beta} \partial_{x}^{\alpha} u$ is well-defined as a tempered distribution. In addition to the classical Sobolev spaces, we also introduce

$$
\begin{aligned}
& W^{1,2, q}(G):=\left\{u \in L^{q}(G) \mid\|u\|_{1,2, q}<\infty\right\}, \\
& \|u\|_{1,2, q}:=\left(\sum_{|\alpha| \leq 2,|\beta| \leq 1}\left\|\partial_{x}^{\alpha} u\right\|_{q}^{q}+\left\|\partial_{t}^{\beta} u\right\|_{q}^{q}\right)^{1 / q} .
\end{aligned}
$$

Standard mollifier arguments yield that $C_{0}^{\infty}(G)$ is dense in both $W^{k, q}(G)$ and $W^{1,2, q}(G)$. Identifying $G$ with $\mathbb{R}^{3} \times[0, \mathcal{T})$, we see that

$$
W^{m, q}(G) \subset W^{m, q}\left(\mathbb{R}^{3} \times[0, \mathcal{T})\right) .
$$

Consequently, classical Sobolev embedding theorems are available for the Sobolev spaces of $G$-defined functions via the inclusion above.

We further define the following subspaces of solenoidal, that is, divergence free, vector fields:

$$
\begin{aligned}
& C_{0, \sigma}^{\infty}(G):=\left\{u \in C_{0}^{\infty}(G)^{3} \mid \operatorname{div} u=0\right\}, \\
& L_{\sigma}^{q}(G):=\overline{C_{0, \sigma}^{\infty}(G)}\|\cdot\|_{q} \\
& W_{\sigma}^{1,2, q}(G):={\overline{C_{0, \sigma}^{\infty}(G)}}^{\|\cdot\|_{1,2, q}} .
\end{aligned}
$$

We have the following characterization of the spaces above:

Lemma 3.2.1. For any $q \in(1, \infty)$ and $k, l \in \mathbb{N}_{0}$ :

$$
\begin{aligned}
& L_{\sigma}^{q}(G)=\left\{u \in L^{q}(G)^{3} \mid \operatorname{div} u=0\right\}, \\
& W_{\sigma}^{1,2, q}(G)=\left\{u \in W^{1,2, q}(G)^{3} \mid \operatorname{div} u=0\right\} .
\end{aligned}
$$

The above identities are well-known if the underlying domain of the function spaces is, for example, $\mathbb{R}^{3}$. A proof can be found in [17, Chapter III.4]. To show the identities in the case where $\mathbb{R}^{3}$ is replaced with $G$, we need only to make some simple modifications to the proof in [17, Chapter III.4]. For sake of completeness, we sketch the modified proof here. 


\section{Linear theory}

Proof of Lemma 3.2.1. Let $\eta: \mathbb{R}^{4} \rightarrow \mathbb{R}$ be a function satisfying

$$
\eta \in C_{0}^{\infty}\left(\mathbb{R}^{4} ; \mathbb{R}\right), \quad \operatorname{supp} \eta \subset \mathrm{B}_{\mathcal{T} / 4}(0), \quad \eta \geq 0, \quad \int_{\mathbb{R}^{4}} \eta(y) \mathrm{d} y=1 .
$$

Let $\tilde{\eta}$ be the restriction of $\eta$ to $\mathbb{R}^{3} \times\left[-\frac{\mathcal{T}}{2}, \frac{\mathcal{T}}{2}\right)$. By identifying $\left[-\frac{\mathcal{T}}{2}, \frac{\mathcal{T}}{2}\right)$ with the torus $\mathbb{T}:=\mathbb{R} / \mathcal{T} \mathbb{Z}$, we can then consider $\tilde{\eta}$ as function $\tilde{\eta}: G \rightarrow \mathbb{R}$. For $0<\varepsilon<1$ we then define

$$
\eta_{\varepsilon} \in C_{0}^{\infty}(G), \quad \eta_{\varepsilon}(x, t):=\frac{1}{\varepsilon^{4}} \eta\left(\frac{1}{\varepsilon} x, \frac{1}{\varepsilon} t\right) .
$$

Clearly, $\eta_{\varepsilon}$ is a mollifier, that is, an approximate identity, on $G$. We also introduce a "cut-off" function $\gamma_{\varepsilon}$ with

$$
\gamma_{\varepsilon} \in C_{0}^{\infty}\left(\mathbb{R}^{3} ; \mathbb{R}\right), \quad \gamma_{\varepsilon}=1 \text { on } \mathrm{B}_{\frac{1}{2 \varepsilon}}(0), \quad \gamma_{\varepsilon}=0 \text { on } \mathbb{R}^{3} \backslash \mathrm{B}_{\frac{1}{\varepsilon}}(0), \quad\left|\nabla \gamma_{\varepsilon}(x)\right| \leq c_{1} \varepsilon .
$$

Now consider $f \in L^{q}(G)^{3}$ with $\operatorname{div} f=0$. In order to establish (3.16), we must show that $f$ can be approximated in the $L^{q}$-norm by functions from $C_{0, \sigma}^{\infty}(G)$. Put

$$
g_{\varepsilon}(x, t):=\gamma_{\varepsilon}(x) \cdot f * \eta_{\varepsilon}(x, t) .
$$

Then $g_{\varepsilon} \in C_{0}^{\infty}(G)^{3}$ and

$$
\operatorname{div} g_{\varepsilon}(x)=\nabla \gamma_{\varepsilon}(x) \cdot f * \eta_{\varepsilon}(x, t) .
$$

Observe that $g_{\varepsilon}$ approximates $f$ in the $L^{q}$-norm as $\varepsilon \rightarrow 0$, but $g_{\varepsilon}$ is not necessarily divergence free. We shall therefore add a correction term to $g_{\varepsilon}$. For this purpose we introduce a "Bogovskil-kernel" $\mathcal{N}$. Let $\omega: \mathbb{R}^{3} \rightarrow \mathbb{R}$ be some function with

$$
\omega \in C_{0}\left(\mathbb{R}^{3} ; \mathbb{R}\right), \quad \operatorname{supp} \omega \subset \mathrm{B}_{1}, \quad \int_{\mathrm{B}_{1}} \omega(y) \mathrm{d} y=1 .
$$

We then define the "Bogovskiur-kernel" $\mathcal{N}$ by

$$
\begin{aligned}
& \mathcal{N}: \mathbb{R}^{3} \times \mathbb{R}^{3} \backslash\{(x, y) \mid x=y\} \rightarrow \mathbb{R}^{3}, \\
& \mathcal{N}(x, y):=\frac{x-y}{|x-y|^{3}} \int_{|x-y|}^{\infty} \omega\left(y+\lambda \frac{x-y}{|x-y|}\right) \lambda^{2} \mathrm{~d} \lambda .
\end{aligned}
$$

The "Bogovskiu-kernel" is used to define

$$
\kappa_{\varepsilon}: G \rightarrow \mathbb{R}, \quad \kappa_{\varepsilon}(x, t):=\int_{\mathbb{R}^{3}} \nabla \gamma_{\varepsilon}(y) \cdot f * \eta_{\varepsilon}(y, t) \mathcal{N}(x, y) \mathrm{d} y .
$$


For $y \in \operatorname{supp} \nabla \gamma_{\varepsilon} \subset \mathrm{B}_{1 / 2 \varepsilon, 1 / \varepsilon}$ the condition $y+\lambda \frac{x-y}{|x-y|} \in \mathrm{B}_{1}$ implies $|\lambda|<1+\frac{1}{\varepsilon}$. Consequently

$$
\left|\nabla \gamma_{\varepsilon}(y)\right||\mathcal{N}(x, y)| \leq c_{2} \frac{\chi_{\mathrm{B}_{1 / 2 \varepsilon, 1 / \varepsilon}}(y)}{|x-y|^{2}} \int_{0}^{1+\frac{1}{\varepsilon}} \lambda^{2} \mathrm{~d} \lambda \leq c_{3} \chi_{\mathrm{B}_{1 / 2 \varepsilon, 1 / \varepsilon}}(y) \frac{1}{|x-y|^{2}},
$$

with $c_{3}=c_{3}(\varepsilon)$. Thus $y \rightarrow\left|\nabla \gamma_{\varepsilon}(y)\right||\mathcal{N}(x, y)|$ has compact support and belongs to $L^{1}\left(\mathbb{R}^{3}\right)$. Consequently, we can differentiate $\kappa_{\varepsilon}$ with respect to time and see that in fact $t \rightarrow \kappa_{\varepsilon}(x, t)$ is smooth. From the properties of $\mathcal{N}$ it is well-known that $x \rightarrow \kappa_{\varepsilon}(x, t)$ is also smooth; see for example [17, Proof of Lemma III.3.1]. It is therefore possible to conclude that $\kappa_{\varepsilon} \in C^{\infty}(G)$. By further well-known properties of the "Bogovskil-kernel" $\mathcal{N}$, see again [17, Proof of Lemma III.3.1], we have

$$
\left\{\begin{array}{l}
\operatorname{div} \kappa_{\varepsilon}(x, t)=\nabla \gamma_{\varepsilon}(x) \cdot f * \eta_{\varepsilon}(x, t), \\
\operatorname{supp} \kappa_{\varepsilon} \subset \mathrm{B}_{1 / \varepsilon}(0) \times \mathbb{R} / \mathcal{T} \mathbb{Z} \\
\left\|\nabla \kappa_{\varepsilon}(\cdot, t)\right\|_{q} \leq c_{4}\left\|\nabla \gamma_{\varepsilon}(\cdot) f * \eta_{\varepsilon}(\cdot, t)\right\|_{q}, \\
\left\|\nabla \kappa_{\varepsilon}(\cdot, t)\right\|_{1, q} \leq c_{5}\left\|\nabla \gamma_{\varepsilon}(\cdot) f * \eta_{\varepsilon}(\cdot, t)\right\|_{1, q},
\end{array}\right.
$$

with $c_{4}$ and $c_{5}$ independent on $\varepsilon$. We now estimate, using first Poincaré's inequality and then (3.18),

$$
\begin{aligned}
\left\|\kappa_{\varepsilon}\right\|_{L^{q}(G)}^{q} & =\int_{\mathbb{T}} \int_{\mathrm{B}_{1 / \varepsilon}}\left|\kappa_{\varepsilon}(x, t)\right|_{q}^{q} \mathrm{~d} x \mathrm{~d} t \\
& \leq c_{6} \int_{\mathbb{T}}\left(\frac{1}{\varepsilon}\right)^{q}\left\|\nabla \kappa_{\varepsilon}(\cdot, t)\right\|_{q}^{q} \mathrm{~d} t \\
& \leq c_{7} \int_{\mathbb{T}}\left(\frac{1}{\varepsilon}\right)^{q}\left\|\nabla \gamma_{\varepsilon}(\cdot) f * \eta_{\varepsilon}(\cdot, t)\right\|_{q}^{q} \mathrm{~d} t \\
& \leq c_{8} \int_{\mathbb{T}} \int_{\mathrm{B}_{1 / 2 \varepsilon, 1 / \varepsilon}}\left|\int_{G} f(y, s) \eta_{\varepsilon}(x-y, t-s) \mathrm{d} y \mathrm{~d} s\right|^{q} \mathrm{~d} x \mathrm{~d} t,
\end{aligned}
$$

where we in the last inequality used that $\left|\nabla \gamma_{\varepsilon}\right| \leq c_{1} \varepsilon$. Employing Hölder's inequality, we then find

$$
\begin{aligned}
\left\|\kappa_{\varepsilon}\right\|_{L^{q}(G)}^{q} & \leq c_{8} \int_{\mathbb{T}} \int_{\mathrm{B}_{1 / 2 \varepsilon, 1 / \varepsilon}} \int_{G}|f(y, s)|^{q} \eta_{\varepsilon}(x-y, t-s) \mathrm{d} y \mathrm{~d} s \mathrm{~d} x \mathrm{~d} t \\
& =c_{8} \int_{G}|f(y, s)|^{q} \int_{\mathbb{T}} \int_{\mathrm{B}_{1 / 2 \varepsilon, 1 / \varepsilon}} \eta_{\varepsilon}(x-y, t-s) \mathrm{d} x \mathrm{~d} t \mathrm{~d} y \mathrm{~d} s,
\end{aligned}
$$

which by the Lebesgue's dominated convergence theorem yields $\lim _{\varepsilon \rightarrow 0}\left\|\kappa_{\varepsilon}\right\|_{L^{q}(G)}=0$. We conclude that

$$
\left\|\left(g_{\varepsilon}-\kappa_{\varepsilon}\right)-f\right\|_{q} \leq\left\|g_{\varepsilon}-f\right\|_{q}+\left\|\kappa_{\varepsilon}\right\|_{q} \rightarrow 0 \quad \text { as } \varepsilon \rightarrow 0 .
$$




\section{Linear theory}

Since $g_{\varepsilon}-\kappa_{\varepsilon} \in C_{0, \sigma}^{\infty}(G)$, we have thereby established (3.16). The characterization (3.17) can be shown in a similar manner.

Finally, we shall define the Helmholtz projection on the Lebesgue space $L^{q}(G)^{3}$ of vector fields defined on $G$. This can be done in two ways. Either we decompose $L^{q}(G)^{3}$ in a similar manner as in Lemma 2.3.4, and define the Helmholtz projection as the projection induced by the decomposition, or we define it via a Fourier multiplier as in (2.6). We choose the latter.

Definition 3.2.2. For $f \in L^{2}(G)^{3}$ we let

$$
\mathcal{P}_{H} f:=\mathcal{F}_{G}^{-1}\left[\left(I-\frac{\xi \otimes \xi}{|\xi|^{2}}\right) \widehat{f}\right]
$$

and call $\mathcal{P}_{H}$ the Helmholtz projection.

It is not immediately clear from the definition of the Helmholtz projection via the Fourier multiplier in (3.19) that $\mathcal{P}_{H} f$ is a real function if $f$ is real. This, however, is a simple consequence of the fact that the multiplier in question is even.

Since the multiplier on the right-hand side in (3.19) is bounded, it is natural to initially define $\mathcal{P}_{H}$ on $L^{2}(G)^{3}$. If namely $f \in L^{2}(G)^{3}$, by Plancherel's theorem, also $\mathcal{P}_{H} f \in$ $L^{2}(G)^{3}$. We state in the following lemma that $\mathcal{P}_{H}$ can be extended to $L^{q}(G)^{3}$, and that it is a projection with the desired properties of a Helmholtz projection.

Lemma 3.2.3. Let $q \in(1, \infty)$. Then $\mathcal{P}_{H}$ extends uniquely to a continuous projection $\mathcal{P}_{H}: L^{q}(G)^{3} \rightarrow L^{q}(G)^{3}$. Moreover, $\mathcal{P}_{H} L^{q}(G)^{3}=L_{\sigma}^{q}(G)$.

Proof. It is easy to see that we can define on $G:=\mathbb{R}^{3} \times \mathbb{R} / \mathcal{T} \mathbb{Z}$ the partial Fourier transforms $\mathcal{F}_{\mathbb{R}^{3}}: \mathscr{S}^{\prime}(G) \rightarrow \mathscr{S}^{\prime}(G)$ and $\mathcal{F}_{\mathbb{R} / \mathcal{T} \mathbb{Z}}: \mathscr{S}^{\prime}(G) \rightarrow \mathscr{S}^{\prime}(G)$ in the canonical way, and that $\mathcal{F}_{G}=\mathcal{F}_{\mathbb{R}^{3}} \circ \mathcal{F}_{\mathbb{R} / \mathcal{T} \mathbb{Z}}$. Consequently, for $f \in L^{2}(G)^{3} \cap L^{q}(G)^{3}$

$$
\mathcal{F}_{G}^{-1}\left[\left(I-\frac{\xi \otimes \xi}{|\xi|^{2}}\right) \widehat{f}\right]=\mathcal{F}_{\mathbb{R}^{3}}^{-1}\left[\left(I-\frac{\xi \otimes \xi}{|\xi|^{2}}\right) \mathcal{F}_{\mathbb{R}^{3}}[f]\right] .
$$

In view of (2.6), it follows that $\left\|\mathcal{P}_{H} f\right\|_{q} \leq\|f\|_{q}$. Thus, $\mathcal{P}_{H}$ extends uniquely to a continuous map $\mathcal{P}_{H}: L^{q}(G)^{3} \rightarrow L^{q}(G)^{3}$. One readily verifies that $\mathcal{P}_{H}$ is a projection, that is, $\mathcal{P}_{H}=\mathcal{P}_{H}^{2}$, and that $\operatorname{div} \mathcal{P}_{H} f=0$. By Lemma 3.2.1, $\mathcal{P}_{H} L^{q}(G)^{3} \subset L_{\sigma}^{q}(G)$ follows. On the other hand, since $\operatorname{div} f=0$ implies $\xi_{j} \widehat{f}_{j}=0$, we have $\mathcal{P}_{H} f=f$ for $f \in L_{\sigma}^{q}(G)$. Hence we conclude $\mathcal{P}_{H} L^{q}(G)^{3}=L_{\sigma}^{q}(G)$.

Since $\mathcal{P}_{H}: L^{q}(G)^{3} \rightarrow L^{q}(G)^{3}$ is a continuous projection, it decomposes $L^{q}(G)$ into a direct sum

$$
L^{q}(G)=L_{\sigma}^{q}(G) \oplus \mathscr{G}^{q}(G)
$$

with

$$
\mathscr{G}^{q}(G):=\left[\operatorname{Id}-\mathcal{P}_{H}\right] L^{q}(G)^{3} .
$$




\subsubsection{Fourier transform}

As previously mentioned, $G:=\mathbb{R}^{3} \times \mathbb{R} / \mathcal{T} \mathbb{Z}$ is a locally compact abelian group. We identify each $(\xi, k) \in \mathbb{R}^{3} \times \mathbb{Z}$ with the character

$$
\chi: G \rightarrow \mathbb{C}, \quad \chi(x, t):=\mathrm{e}^{i x \cdot \xi+i k \frac{2 \pi}{\mathcal{T}} t}
$$

on $G$. It is standard to verify all are characters are of this form, and we can thus identify

$$
\widehat{G}=\mathbb{R}^{3} \times \mathbb{Z} .
$$

One readily checks that the compact-open topology on $\widehat{G}$ coincides with the product of the Euclidean topology on $\mathbb{R}^{3}$ and the discrete topology on $\mathbb{Z}$.

The Fourier transform $\mathcal{F}_{G}$ on $G$ is given by

$$
\mathcal{F}_{G}: L^{1}(G) \rightarrow C(\widehat{G}), \quad \mathcal{F}_{G}(u)(\xi, k):=\widehat{u}(\xi, k):=\frac{1}{\mathcal{T}} \int_{0}^{\mathcal{T}} \int_{\mathbb{R}^{3}} u(x, t) \mathrm{e}^{-i x \cdot \xi-i k \frac{2 \pi}{\mathcal{T}} t} \mathrm{~d} x \mathrm{~d} t .
$$

If no confusion can arise, we simply write $\mathcal{F}$ instead of $\mathcal{F}_{G}$.

We now introduce the generalized Schwartz-Bruhat space corresponding to the dual group $\widehat{G}$. More specifically, we let

$$
\forall \alpha, \beta \in \mathbb{N}_{0}^{3}, \kappa \in \mathbb{N}_{0}: \hat{\rho}_{\alpha, \beta, \kappa}(w):=\sup _{(\xi, k) \in \widehat{G}}\left|k^{\kappa}\right|\left|\xi^{\alpha}\right|\left|\partial_{\xi}^{\beta} w(\xi, k)\right|,
$$

and put

$$
\begin{aligned}
\mathscr{S}(\widehat{G}):=\{w \in C(\widehat{G}) \mid & \forall k \in \mathbb{Z}: w(\cdot, k) \in C^{\infty}\left(\mathbb{R}^{3}\right), \\
& \left.\forall \alpha, \beta \in \mathbb{N}_{0}^{3}, \kappa \in \mathbb{N}_{0}: \hat{\rho}_{\alpha, \beta, \kappa}(w)<\infty\right\} .
\end{aligned}
$$

Clearly, $\mathscr{S}(\widehat{G})$ is a vector space. We endow $\mathscr{S}(\widehat{G})$ with the semi-norm topology induced by the family of semi-norms $\left\{\hat{\rho}_{\alpha, \beta, \kappa} \mid \alpha, \beta \in \mathbb{N}_{0}^{3}, \kappa \in \mathbb{N}_{0}\right\}$. The topological dual space of $\mathscr{S}(\widehat{G})$ is denoted by $\mathscr{S}^{\prime}(\widehat{G})$. We equip $\mathscr{S}^{\prime}(\widehat{G})$ with the weak* topology. We can define $\mathscr{S}(\widehat{G})$ and $\mathscr{S}^{\prime}(\widehat{G})$ both as real and complex vector spaces, but will only use then as complex vector spaces in the following.

We observe that $\mathscr{S}(\widehat{G})$ is closed under multiplication by finite polynomials of type $p(\xi, k):=\sum_{\alpha \in \mathbb{N}_{0}^{3}, \kappa \in \mathbb{N}_{0}} a_{\alpha, \kappa} \xi^{\alpha} k^{\kappa}$. By duality, $\mathscr{S}^{\prime}(\widehat{G})$ also remains closed under multiplication by such polynomials. Similarly, both $\mathscr{S}(\widehat{G})$ and $\mathscr{S}^{\prime}(\widehat{G})$ remain closed under multiplication by functions that are smooth in the $\xi$-variable and have at most polynomial growth with respect to both $\xi$ and $k$. In particular, $\mathscr{S}(\widehat{G})$ and $\mathscr{S}^{\prime}(\widehat{G})$ remain closed under multiplication by elements $\psi \in \mathscr{S}(\widehat{G})$.

It is easy to verify that

$$
\mathcal{F}: \mathscr{S}(G) \rightarrow \mathscr{S}(\widehat{G})
$$




\section{Linear theory}

continuously. The inverse Fourier transform is formally defined by

$$
\mathcal{F}^{-1}: L^{1}(\widehat{G}) \rightarrow C(G), \quad \mathcal{F}^{-1}(w)(x, t):=w^{\vee}(x, t):=\sum_{k \in \mathbb{Z}} \int_{\mathbb{R}^{3}} w(\xi, k) \mathrm{e}^{i x \cdot \xi+i k \frac{2 \pi}{\mathcal{T}} t} \mathrm{~d} \xi .
$$

One readily checks that

$$
\mathcal{F}^{-1}: \mathscr{S}(\widehat{G}) \rightarrow \mathscr{S}(G)
$$

continuously. Provided the Lebesgue measure $\mathrm{d} \xi$ is normalized appropriately, it is standard to show that $\mathcal{F}^{-1}$ is the inverse of the mapping (3.22). Thus, the Fourier transform $\mathcal{F}: \mathscr{S}(G) \rightarrow \mathscr{S}(\widehat{G})$ is a homeomorphism.

By duality, $\mathcal{F}$ extends to a mapping $\mathscr{S}^{\prime}(G) \rightarrow \mathscr{S}^{\prime}(\widehat{G})$. More precisely,

$$
\mathcal{F}: \mathscr{S}^{\prime}(G) \rightarrow \mathscr{S}^{\prime}(\widehat{G}), \quad \forall \psi \in \mathscr{S}(\widehat{G}):\langle\mathcal{F}(u), \psi\rangle:=\langle u, \mathcal{F}(\psi)\rangle .
$$

Similarly,

$$
\mathcal{F}^{-1}: \mathscr{S}^{\prime}(\widehat{G}) \rightarrow \mathscr{S}^{\prime}(G), \quad \forall \psi \in \mathscr{S}(G):\left\langle\mathcal{F}^{-1}(u), \psi\right\rangle:=\left\langle u, \mathcal{F}^{-1}(\psi)\right\rangle .
$$

Clearly,

$$
\mathcal{F}: \mathscr{S}^{\prime}(G) \rightarrow \mathscr{S}^{\prime}(\widehat{G})
$$

homeomorphically. To avoid confusion, we shall sometimes denote the above Fourier transform and its inverse by $\mathcal{F}_{G}$ and $\mathcal{F}_{G}^{-1}$, respectively.

The Fourier transform in the setting above provides us with a calculus between differential operators on $G$ and the polynomials on $\widehat{G}$. As one easily verifies, for $u \in \mathscr{S}^{\prime}(G)$ and $\alpha \in \mathbb{N}_{0}^{3}, l \in \mathbb{N}_{0}$ we have

$$
\mathcal{F}\left(\partial_{t}^{l} \partial_{x}^{\alpha} u\right)=i^{l+|\alpha|}\left(\frac{2 \pi}{\mathcal{T}}\right)^{l} k^{l} \xi^{\alpha} \mathcal{F}(u)
$$

as an identity in $\mathscr{S}^{\prime}(\widehat{G})$.

\subsubsection{Reformulation}

Since the topology and differentiable structure on $G$ is inherited from $\mathbb{R}^{3} \times \mathbb{R}$, we obtain the following equivalent formulation of (3.1) as a system over $G$-defined vector fields:

$$
\begin{cases}\partial_{t} u-\Delta u-\lambda \partial_{1} u+\nabla \mathfrak{p}=f & \text { in } G, \\ \operatorname{div} u=0 & \text { in } G, \\ \lim _{|x| \rightarrow \infty} u(x, t)=0 & \end{cases}
$$

with unknowns

$$
u: G \rightarrow \mathbb{R}^{3}, \quad \mathfrak{p}: G \rightarrow \mathbb{R}
$$


and data

$$
f: G \rightarrow \mathbb{R}^{3}
$$

Observe that in this formulation the periodicity condition $u(x, t+\mathcal{T})=u(x, t)$ is not needed anymore. On the same token, we do not need to require time periodicity of the data either. Indeed, all functions defined on $G$ are by definition time-periodic with period $\mathcal{T}$.

We shall investigate (3.24) in an $L^{q}(G)$-setting, that is, we will study the resolvability of (3.24) for data $f \in L^{q}(G)^{3}$. In this context, the Sobolev spaces defined in Section 3.2.2 are used to describe the corresponding function spaces of the solution. Employing the Fourier transformation introduced in Section 3.2.3, we will be able to show the required estimates via a multiplier ansatz.

\subsection{Representation formula}

If we apply the Fourier transform $\mathcal{F}_{G}$ on both sides of the equations in (3.24), we obtain the equivalent system ${ }^{1}$

$$
\begin{cases}\left(i \frac{2 \pi}{\mathcal{T}} k\right) \widehat{u}+|\xi|^{2} \widehat{u}-\lambda i \xi_{1} \widehat{u}+i \widehat{\mathfrak{p}} \xi=\widehat{f} & \text { in } \widehat{G}, \\ \xi_{j} \widehat{u}_{j}=0 & \text { in } \widehat{G}, \\ \lim _{|x| \rightarrow \infty} u(x, t)=0 . & \end{cases}
$$

Dot-multiplying the first equation with $\xi$, we obtain the relation

$$
-\xi_{h} \xi_{h} \widehat{\mathfrak{p}}=i \xi_{j} \widehat{f}_{j}
$$

and thus

$$
\left(\left(i \frac{2 \pi}{\mathcal{T}} k\right)+|\xi|^{2}-\lambda i \xi_{1}\right) \widehat{u}=\left(I-\frac{\xi \otimes \xi}{|\xi|^{2}}\right) \widehat{f}
$$

Formally, we can therefore deduce

$$
u=\mathcal{F}_{G}^{-1}\left[\frac{1}{\left(i \frac{2 \pi}{\mathcal{T}} k\right)+|\xi|^{2}-\lambda i \xi_{1}}\left(I-\frac{\xi \otimes \xi}{|\xi|^{2}}\right) \widehat{f}\right] .
$$

Since the multiplier

$$
(\xi, k) \rightarrow \frac{1}{\left(i \frac{2 \pi}{\mathcal{T}} k\right)+|\xi|^{2}-\lambda i \xi_{1}}\left(I-\frac{\xi \otimes \xi}{|\xi|^{2}}\right)
$$

is well-defined as an element of $\mathscr{S}^{\prime}(\widehat{G})$, the representation formula (3.28) is valid at the outset as an identity in $\mathscr{S}^{\prime}(G)$ for all $f \in \mathscr{S}(G)$.

\footnotetext{
${ }^{1}$ We make use of the Einstein summation convention and implicitly sum over all repeated indices.
} 


\section{Linear theory}

\subsection{Transference of multipliers}

We shall utilize (3.28) to estimate $u$ in terms of the $L^{q}(G)$-norm of $f$. Since (3.28) is a representation of $u$ by means of a Fourier multiplier, we shall seek to use standard theory of Fourier multipliers to obtain the estimates. However, standard multiplier theory such as the theorems of Mihlin, Lizorkin, and Marcinkiewicz only applies to multipliers in $\mathbb{R}^{n}$. In order to nevertheless employ these powerful theorems, we will use a so-called transference principle for Fourier multipliers which enables us to "transfer" multipliers from one group setting into a different group setting. More precisely, we shall "transfer" the $G$-multiplier from (3.28) into a $\mathbb{R}^{4}$-setting, then analyze the multiplier in this setting using one of the standard multiplier theorems, and finally "transfer" the resulting estimates back to the $G$-setting. The transference principle is expressed in following theorem. The theorem is originally due to DE LEEUW [4]; the version below is due to EDWARDS and GAUDRY [5].

Theorem 3.4.1. Let $G$ and $H$ be locally compact abelian groups. Moreover, let

$$
\Phi: \widehat{G} \rightarrow \widehat{H}
$$

be a continuous homomorphism and $q \in[1, \infty]$. Assume that $m \in L^{\infty}(\widehat{H} ; \mathbb{C})$ is a continuous $L^{q}$-multiplier, that is, there is a constant $C_{5}$ such that

$$
\forall f \in L^{2}(H) \cap L^{q}(H):\left\|\mathcal{F}_{H}^{-1}[m \cdot \widehat{f}]\right\|_{q} \leq C_{5}\|f\|_{q} .
$$

Then $m \circ \Phi \in L^{\infty}(\widehat{G} ; \mathbb{C})$ is also an $L^{q}$-multiplier with

$$
\forall f \in L^{2}(G) \cap L^{q}(G):\left\|\mathcal{F}_{G}^{-1}[m \circ \Phi \cdot \widehat{f}]\right\|_{q} \leq C_{5}\|f\|_{q} .
$$

Proof. See Theorem B.2.1 and the proof hereof in [5].

Theorem 3.4.1 will play a fundamental role in the following, where we shall employ it with $G:=\mathbb{R}^{3} \times \mathbb{R} / \mathcal{T} \mathbb{Z}, H:=\mathbb{R}^{3} \times \mathbb{R}$, and $\Phi: \mathbb{R}^{3} \times \mathbb{Z} \rightarrow \mathbb{R}^{3} \times \mathbb{R}, \Phi(\xi, k):=\left(\xi, \frac{2 \pi}{\mathcal{T}} k\right)$. For the sake of completeness, a full proof of this special case of the theorem will be given below. We shall follow the proof of [28, Theorem 3.6.7]. We start with a lemma.

Lemma 3.4.2. Let $q \in(1, \infty)$. Then

$$
\mathcal{C}_{\text {per }}:=\operatorname{span}\left\{\varphi(x) \mathrm{e}^{i \frac{2 \pi}{\mathcal{T}} k t} \mid \varphi \in C_{0}^{\infty}\left(\mathbb{R}^{3}\right), k \in \mathbb{Z}\right\}
$$

is a dense subset of $L^{q}\left(\mathbb{R}^{3} \times \mathbb{R} / \mathcal{T} \mathbb{Z} ; \mathbb{C}\right)$.

Proof. By a standard argument based on mollifiers, see for example the proof of Lemma 3.2 .1 , it is easy to show that $C_{0}^{\infty}\left(\mathbb{R}^{3} \times \mathbb{R} / \mathcal{T} \mathbb{Z}\right)$ a dense subset of $L^{q}\left(\mathbb{R}^{3} \times \mathbb{R} / \mathcal{T} \mathbb{Z}\right)$. It is therefore enough to show that any $\Psi \in C_{0}^{\infty}\left(\mathbb{R}^{3} \times \mathbb{R} / \mathcal{T} \mathbb{Z}\right)$ can be approximated in the $L^{q}\left(\mathbb{R}^{3} \times \mathbb{R} / \mathcal{T} \mathbb{Z}\right)$-norm by functions from $\mathcal{C}_{\text {per }}$. For this purpose we expand $\Psi(x, t)$ in a Fourier series with respect to the $t$-variable. We then have

$$
\Psi(x, t)=\sum_{k \in \mathbb{Z}} \Psi_{k}(x) \mathrm{e}^{i \frac{2 \pi}{\mathcal{T}} k t}, \quad \Psi_{k}(x):=\int_{\mathbb{R} / \mathcal{T} \mathbb{Z}} \Psi(x, t) \mathrm{e}^{-i \frac{\mathcal{T}}{2 \pi} k t} \mathrm{~d} t .
$$


Clearly $\Psi_{k} \in C_{0}^{\infty}\left(\mathbb{R}^{3}\right)$. We now claim that $\sum_{|k| \leq N} \Psi_{k}(x) \mathrm{e}^{i \frac{2 \pi}{T} k t} \in \mathcal{C}_{\text {per }}$ converges in the $L^{q}\left(\mathbb{R}^{3} \times \mathbb{R} / \mathcal{T} \mathbb{Z}\right)$-norm to $\Psi$ as $N \rightarrow \infty$. In fact, employing twice Minkowski's integral inequality we see that

$$
\begin{aligned}
\left\|\Psi-\sum_{|k| \leq N} \Psi_{k}(x) \mathrm{e}^{i \frac{2 \pi}{\mathcal{T}} k t}\right\|_{q} & =\left(\int_{\mathbb{R}^{3}} \int_{\mathbb{R} / \mathcal{T} \mathbb{Z}}\left|\sum_{|k|>N} \Psi_{k}(x) \mathrm{e}^{i \frac{2 \pi}{\mathcal{T}} k t}\right|^{q} \mathrm{~d} t \mathrm{~d} x\right)^{\frac{1}{q}} \\
& \leq \sum_{|k|>N}\left(\int_{\mathbb{R}^{3}}\left|\Psi_{k}(x)\right|^{q} \mathrm{~d} x\right)^{\frac{1}{q}} \\
& \leq \sum_{|k|>N}\left(\int_{\mathbb{R}^{3}}\left|\int_{\mathbb{R} / \mathcal{T} \mathbb{Z}} \Psi(x, t) \mathrm{e}^{-i \frac{\mathcal{T}}{2 \pi} k t} \mathrm{~d} t\right|^{q} \mathrm{~d} x\right)^{\frac{1}{q}} \\
& \leq \sum_{|k|>N} \frac{c_{1}}{k^{2}}\left\|\partial_{t}^{2} \Psi(x, t)\right\|_{q},
\end{aligned}
$$

from which the convergence follows.

The following lemma is a modified version of [28, Lemma 3.6.8].

Lemma 3.4.3. Let $\alpha \in(0,1)$ and $q \in(1, \infty)$. For a function $m \in L^{\infty}\left(\mathbb{R}^{3} \times \mathbb{R} ; \mathbb{C}\right)$ define $M: \mathbb{R}^{3} \times \mathbb{Z} \rightarrow \mathbb{C}$ by $M(\xi, k):=m\left(\xi, \frac{2 \pi}{\mathcal{T}} k\right)$. If $m$ is continuous, then

$$
\begin{aligned}
\forall \varphi, \psi \in C_{0}^{\infty}\left(\mathbb{R}^{3}\right) \forall k, l \in \mathbb{Z}: \\
\lim _{\varepsilon \rightarrow 0} \varepsilon^{\frac{1}{2}} \int_{\mathbb{R}^{3}} \int_{\mathbb{R}} \mathcal{F}_{\mathbb{R}^{3} \times \mathbb{R}}^{-1}\left[m \mathcal{F}_{\mathbb{R}^{3} \times \mathbb{R}}\left[\varphi(x) \mathrm{e}^{i \frac{2 \pi}{\mathcal{T}} k t} \mathrm{e}^{-\alpha \varepsilon t^{2}}\right]\right] \cdot \psi(x) \mathrm{e}^{i \frac{2 \pi}{\mathcal{T}} l t} \mathrm{e}^{-(1-\alpha) \varepsilon t^{2}} \mathrm{~d} t \mathrm{~d} x \\
\quad=\int_{\mathbb{R}^{3}} \int_{\mathbb{R} / \mathcal{T} \mathbb{Z}} \mathcal{F}_{\mathbb{R}^{3} \times \mathbb{R} / \mathcal{T} \mathbb{Z}}^{-1}\left[M \mathcal{F}_{\mathbb{R}^{3} \times \mathbb{R} / \mathcal{T} \mathbb{Z}}\left[\varphi(x) \mathrm{e}^{i \frac{2 \pi}{\mathcal{T}} k t}\right]\right] \cdot \psi(x) \mathrm{e}^{i \frac{2 \pi}{\mathcal{T}} l t} \mathrm{~d} t \mathrm{~d} x .
\end{aligned}
$$

Proof. Let $\varphi, \psi \in C_{0}^{\infty}\left(\mathbb{R}^{3}\right)$ and $k, l \in \mathbb{Z}$. Employing Parseval's identity, we find that

$$
\begin{aligned}
\varepsilon^{\frac{1}{2}} \int_{\mathbb{R}^{3}} \int_{\mathbb{R}} \mathcal{F}_{\mathbb{R}^{3} \times \mathbb{R}}^{-1}\left[m \mathcal{F}_{\mathbb{R}^{3} \times \mathbb{R}}\left[\varphi(x) \mathrm{e}^{i \frac{2 \pi}{\mathcal{T}} k t} \mathrm{e}^{-\alpha \varepsilon t^{2}}\right]\right] \cdot \psi(x) \mathrm{e}^{i \frac{2 \pi}{\mathcal{T}} l t} \mathrm{e}^{-(1-\alpha) \varepsilon t^{2}} \mathrm{~d} t \mathrm{~d} x \\
=\varepsilon^{\frac{1}{2}} \int_{\mathbb{R}^{3}} \int_{\mathbb{R}} m(\xi, \eta) \mathcal{F}_{\mathbb{R}^{3} \times \mathbb{R}}\left[\varphi(x) \mathrm{e}^{i \frac{2 \pi}{\mathcal{T}} k t} \mathrm{e}^{-\alpha \varepsilon t^{2}}\right] \cdot \mathcal{F}_{\mathbb{R}^{3} \times \mathbb{R}}\left[\psi(x) \mathrm{e}^{i \frac{2 \pi}{\mathcal{T}} l t} \mathrm{e}^{-(1-\alpha) \varepsilon t^{2}}\right] \mathrm{d} \eta \mathrm{d} \xi \\
=\varepsilon^{-\frac{1}{2}}(\alpha(1-\alpha))^{-\frac{1}{2}} \int_{\mathbb{R}^{3}} \int_{\mathbb{R}} m(\xi, \eta) \widehat{\varphi}(\xi) \widehat{\psi}(\xi) \mathrm{e}^{-\frac{\left|\eta-\frac{2 \pi}{T} k\right|^{2}}{\varepsilon \alpha}} \mathrm{e}^{-\frac{\left|\eta-\frac{2 \pi}{T} l\right|^{2}}{\varepsilon(1-\alpha)}} \mathrm{d} \eta \mathrm{d} \xi .
\end{aligned}
$$




\section{Linear theory}

If $k=l$, the right-hand side above equals

$$
\int_{\mathbb{R}^{3}} \widehat{\varphi}(\xi) \widehat{\psi}(\xi) \int_{\mathbb{R}} m(\xi, \eta)(\varepsilon \alpha(1-\alpha))^{-\frac{1}{2}} \mathrm{e}^{-\frac{\left|\eta-\frac{2 \pi}{T} k\right|^{2}}{\varepsilon \alpha(1-\alpha)}} \mathrm{d} \eta \mathrm{d} \xi .
$$

We now recall the well-known fact that $\eta \rightarrow \varepsilon^{-\frac{1}{2}} \mathrm{e}^{-\frac{\eta^{2}}{\varepsilon}}$ is an approximate identity on $\mathbb{R}$. Employing Lebesgue's dominated convergence theorem and exploiting that $m$ is continuous, we thus obtain

$$
\begin{aligned}
\lim _{\varepsilon \rightarrow 0} \int_{\mathbb{R}^{3}} \widehat{\varphi}(\xi) \widehat{\psi}(\xi) \int_{\mathbb{R}} m(\xi, \eta)(\varepsilon \alpha(1-\alpha))^{-\frac{1}{2}} \mathrm{e}^{-\frac{\left|\eta-\frac{2 \pi}{\mathcal{T}} k\right|^{2}}{\varepsilon \alpha(1-\alpha)}} \mathrm{d} \eta \mathrm{d} \xi \\
\quad=\int_{\mathbb{R}^{3}} \widehat{\varphi}(\xi) \widehat{\psi}(\xi) m\left(\xi, \frac{2 \pi}{\mathcal{T}} k\right) \mathrm{d} \xi
\end{aligned}
$$

If, on the other hand, $k \neq l$, then the integral on the right-hand side in (3.31) can be estimated by

$$
\begin{gathered}
\varepsilon^{-\frac{1}{2}}(\alpha(1-\alpha))^{-\frac{1}{2}}\left|\int_{\mathbb{R}^{3}} \int_{\mathbb{R}} m(\xi, \eta) \widehat{\varphi}(\xi) \widehat{\psi}(\xi) \mathrm{e}^{-\frac{\left|\eta-\frac{2 \pi}{T} k\right|^{2}}{\varepsilon \alpha}} \mathrm{e}^{-\frac{\mid \eta-\frac{2 \pi}{T} l l^{2}}{\varepsilon(1-\alpha)}} \mathrm{d} \eta \mathrm{d} \xi\right| \\
\leq c_{1}\|m\|_{\infty} \varepsilon^{-\frac{1}{2}} \int_{\mathbb{R}^{3}}\left(\int_{\left\{\left|\eta-\frac{2 \pi}{T} l\right|>\frac{\pi}{T}\right\}}|\widehat{\varphi}(\xi)||\widehat{\psi}(\xi)| \mathrm{e}^{-\frac{1}{4 \varepsilon(1-\alpha)}} \mathrm{e}^{-\frac{\left|\eta-\frac{2 \pi}{T} k\right|^{2}}{\varepsilon \alpha}} \mathrm{d} \eta\right. \\
\left.+\int_{\left\{\left|\eta-\frac{2 \pi}{T} k\right|>\frac{\pi}{T}\right\}}|\widehat{\varphi}(\xi)||\widehat{\psi}(\xi)| \mathrm{e}^{-\frac{1}{4 \varepsilon \alpha}} \mathrm{e}^{-\frac{\left.\left|\eta-\frac{2 \pi}{\tau} l\right|\right|^{2}}{\varepsilon(1-\alpha)}} \mathrm{d} \eta\right) \mathrm{d} \xi \\
\leq c_{2}\|m\|_{\infty}\left(\mathrm{e}^{-\frac{1}{4 \varepsilon(1-\alpha)}}+\mathrm{e}^{-\frac{1}{4 \varepsilon \alpha}}\right) \int_{\mathbb{R}^{3}}|\widehat{\varphi}(\xi) \| \widehat{\psi}(\xi)| \mathrm{d} \xi \\
\rightarrow 0 \quad \text { as } \varepsilon \rightarrow 0 .
\end{gathered}
$$

Combining (3.31), (3.32), and (3.33), we see that the left-hand side in (3.30) equals

$$
\begin{cases}\int_{\mathbb{R}^{3}} \widehat{\varphi}(\xi) \widehat{\psi}(\xi) m\left(\xi, \frac{2 \pi}{\mathcal{T}} k\right) \mathrm{d} \xi & \text { if } k=l \\ 0 & \text { if } k \neq l .\end{cases}
$$

Employing again Parseval's identity, we see that the right-hand side in (3.30) equals

$$
\int_{\mathbb{R}^{3}} \sum_{h \in \mathbb{Z}} M(\xi, h) \widehat{\varphi}(\xi) \delta_{k}(h) \widehat{\psi}(\xi) \delta_{l}(h) \mathrm{d} \xi,
$$

where $\delta_{k}$ and $\delta_{l}$ denotes the Kronecker delta function on $\mathbb{Z}$ with respect to $k$ and $l$, respectively. Clearly, (3.35) and (3.34) coincide, which concludes the lemma. 
The following identity is fundamental in the transference of multipliers between $\mathbb{R}$ and $\mathbb{R} / \mathcal{T} \mathbb{Z}$.

Lemma 3.4.4. If $F \in L^{1}(\mathbb{R} / \mathcal{T} \mathbb{Z})$ then

$$
\lim _{\varepsilon \rightarrow 0} \varepsilon^{\frac{1}{2}} \int_{\mathbb{R}} F \circ \pi(t) \mathrm{e}^{-\varepsilon t^{2}} \mathrm{~d} t=\int_{\mathbb{R} / \mathcal{T} \mathbb{Z}} F(t) \mathrm{d} t .
$$

Proof. Observe that

$$
\varepsilon^{\frac{1}{2}} \int_{\mathbb{R}} F \circ \pi(t) \mathrm{e}^{-\varepsilon t^{2}} \mathrm{~d} t=\varepsilon^{\frac{1}{2}} \sum_{k \in \mathbb{Z}} \int_{0}^{\mathcal{T}} F \circ \pi(t-k \mathcal{T}) \mathrm{e}^{-\varepsilon(t-k \mathcal{T})^{2}} \mathrm{~d} t .
$$

Since

$$
\int_{0}^{\mathcal{T}}\left|\sum_{k \in \mathbb{Z}} \mathrm{e}^{-\varepsilon(t-k \mathcal{T})^{2}}\right| \mathrm{d} t=\int_{\mathbb{R}} \mathrm{e}^{-\varepsilon t^{2}} \mathrm{~d} t<\infty
$$

the function

$$
H: \mathbb{R} / \mathcal{T} \mathbb{Z} \rightarrow \mathbb{R}, \quad H(t):=\sum_{k \in \mathbb{Z}} \varepsilon^{\frac{1}{2}} \mathrm{e}^{-\varepsilon(t-k \mathcal{T})^{2}}
$$

belongs to $L^{1}(\mathbb{R} / \mathcal{T} \mathbb{Z})$. In fact $H \in C^{\infty}(\mathbb{R} / \mathcal{T} \mathbb{Z})$, and the Fourier coefficients $H_{l}$ of $H$ can be estimated, after integration by parts, by

$$
\left|H_{l}\right| \leq\left|\frac{1}{\mathcal{T}} \int_{0}^{\mathcal{T}} H(t) \mathrm{e}^{-i \frac{2 \pi}{\mathcal{T}} l t} \mathrm{~d} t\right| \leq c_{1} l^{-2} \int_{\mathbb{R}}\left(1+t^{2}\right) \mathrm{e}^{-\varepsilon t^{2}} \mathrm{~d} t \leq c_{2} l^{-2} .
$$

Hence $\left\{H_{l}\right\}_{l \in \mathbb{Z}} \in \ell^{1}(\mathbb{Z})$. We can thus utilize both Fubini's theorem and the Fourier inversion formula in (3.37) to deduce that

$$
\begin{aligned}
\varepsilon^{\frac{1}{2}} \int_{\mathbb{R}} F \circ \pi(t) \mathrm{e}^{-\varepsilon t^{2}} \mathrm{~d} t & =\int_{0}^{\mathcal{T}} F(t) H(t) \mathrm{d} t=\int_{0}^{\mathcal{T}} F(t) \sum_{l \in \mathbb{Z}} H_{l} \mathrm{e}^{i \frac{2 \pi}{\mathcal{T}} l t} \mathrm{~d} t \\
& =\int_{0}^{\mathcal{T}} F(t) \sum_{l \in \mathbb{Z}}\left(\frac{1}{\mathcal{T}} \int_{0} \sum_{k \in \mathbb{Z}} \varepsilon^{\frac{1}{2}} \mathrm{e}^{-\varepsilon(s-k \mathcal{T})^{2}} \mathrm{e}^{-i \frac{2 \pi}{\mathcal{T}} l s} \mathrm{~d} s\right) \mathrm{e}^{i \frac{2 \pi}{\mathcal{T}} l t} \mathrm{~d} t \\
& =\int_{\mathbb{R} / \mathcal{T} \mathbb{Z}} F(t) \sum_{l \in \mathbb{Z}}\left(\int_{\mathbb{R}} \varepsilon^{\frac{1}{2}} \mathrm{e}^{-\varepsilon s^{2}} \mathrm{e}^{-i \frac{2 \pi}{\mathcal{T}} l s} \mathrm{~d} s\right) \mathrm{e}^{i \frac{2 \pi}{\mathcal{T}} l t} \mathrm{~d} t \\
& =\int_{\mathbb{R} / \mathcal{T} \mathbb{Z}} F(t) \sum_{l \in \mathbb{Z}} \mathrm{e}^{-\left(\frac{2 \pi}{\mathcal{T}} l\right)^{2} / \varepsilon} \mathrm{e}^{i \frac{2 \pi}{\mathcal{T}} l t} \mathrm{~d} t \\
& =\int_{\mathbb{R} / \mathcal{T} \mathbb{Z}} F(t) \mathrm{d} t+\int_{\mathbb{R} / \mathcal{T} \mathbb{Z}} F(t) \sum_{l \neq 0} \mathrm{e}^{-\left(\frac{2 \pi}{\mathcal{T}} l\right)^{2} / \varepsilon} \mathrm{e}^{i \frac{2 \pi}{\mathcal{T}} l t} \mathrm{~d} t .
\end{aligned}
$$




\section{Linear theory}

Since

$$
\left|\int_{\mathbb{R} / \mathcal{T} \mathbb{Z}} F(t) \sum_{l \neq 0} \mathrm{e}^{-\left(\frac{2 \pi}{\mathcal{T}} l\right)^{2} / \varepsilon} \mathrm{e}^{i \frac{2 \pi}{\mathcal{T}} l t} \mathrm{~d} t\right| \leq\left(\int_{\mathbb{R} / \mathcal{T} \mathbb{Z}}|F(t)| \mathrm{d} t\right) \sum_{l \neq 0} \mathrm{e}^{-\left(\frac{2 \pi}{\mathcal{T}} l\right)^{2} / \varepsilon} \rightarrow 0 \quad \text { as } \varepsilon \rightarrow 0,
$$

the identity (3.36) follows.

We can finally establish the following special case of Theorem 3.4.1.

Theorem 3.4.5. Let $q \in(1, \infty)$. Assume that $m \in L^{\infty}\left(\mathbb{R}^{3} \times \mathbb{R} ; \mathbb{C}\right)$ is a continuous $L^{q}\left(\mathbb{R}^{3} \times \mathbb{R}\right)$-multiplier, that is, there is a constant $C_{6}$ such that

$$
\forall f \in L^{2}\left(\mathbb{R}^{3} \times \mathbb{R}\right) \cap L^{q}\left(\mathbb{R}^{3} \times \mathbb{R}\right):\left\|\mathcal{F}_{\mathbb{R}^{3} \times \mathbb{R}}^{-1}[m \cdot \widehat{f}]\right\|_{q} \leq C_{6}\|f\|_{q} .
$$

Then $M: \mathbb{R}^{3} \times \mathbb{Z} \rightarrow \mathbb{C}, M(\xi, k):=m\left(\xi, \frac{2 \pi}{\mathcal{T}} k\right)$ is an $L^{q}\left(\mathbb{R}^{3} \times \mathbb{R} / \mathcal{T} \mathbb{Z}\right)$-multiplier with

$$
\forall f \in L^{2}\left(\mathbb{R}^{3} \times \mathbb{R} / \mathcal{T} \mathbb{Z}\right) \cap L^{q}\left(\mathbb{R}^{3} \times \mathbb{R} / \mathcal{T} \mathbb{Z}\right):\left\|\mathcal{F}_{\mathbb{R}^{3} \times \mathbb{R} / \mathcal{T} \mathbb{Z}}^{-1}[M \cdot \widehat{f}]\right\|_{q} \leq C_{6}\|f\|_{q}
$$

Proof. It is enough to show

$$
\begin{aligned}
\forall f \in L^{q}\left(\mathbb{R}^{3} \times \mathbb{R} / \mathcal{T} \mathbb{Z}\right), g \in L^{q^{\prime}}\left(\mathbb{R}^{3} \times \mathbb{R} / \mathcal{T} \mathbb{Z}\right): \\
\qquad\left|\int_{\mathbb{R}^{3}} \int_{\mathbb{R} / \mathcal{T} \mathbb{Z}} \mathcal{F}^{-1}[M \cdot \widehat{f}] \cdot g \mathrm{~d} x \mathrm{~d} t\right| \leq C_{6}\|f\|_{q}\|g\|_{q^{\prime}},
\end{aligned}
$$

with $q^{\prime}:=q /(q-1)$ the Hölder conjugate of $q$. By Lemma 3.4.2 if suffices to establish (3.38) for all $f, g \in \mathcal{C}_{\text {per }}$. For such functions Lemma 3.4.3 yields

$$
\begin{aligned}
& \left|\int_{\mathbb{R}^{3}} \int_{\mathbb{R} / \mathcal{T} \mathbb{Z}} \mathcal{F}^{-1}[M \cdot \widehat{f}] \cdot g \mathrm{~d} x \mathrm{~d} t\right| \\
& \quad=\left|\lim _{\varepsilon \rightarrow 0} \varepsilon^{\frac{1}{2}} \int_{\mathbb{R}^{3}} \int_{\mathbb{R}} \mathcal{F}_{\mathbb{R}^{3} \times \mathbb{R}}^{-1}\left[m \mathcal{F}_{\mathbb{R}^{3} \times \mathbb{R}}\left[f \mathrm{e}^{-\frac{1}{q} \varepsilon t^{2}}\right]\right] \cdot g \mathrm{e}^{-\frac{1}{q^{\prime}} \varepsilon t^{2}} \mathrm{~d} t \mathrm{~d} x\right| \\
& \quad \leq C_{6} \lim _{\varepsilon \rightarrow 0} \varepsilon^{\frac{1}{2}}\left(\int_{\mathbb{R}^{3}} \int_{\mathbb{R}}|f|^{q} \mathrm{e}^{-\varepsilon t^{2}} \mathrm{~d} t \mathrm{~d} x\right)^{\frac{1}{q}}\left(\int_{\mathbb{R}^{3}} \int_{\mathbb{R}}|g|^{q^{\prime}} \mathrm{e}^{-\varepsilon t^{2}} \mathrm{~d} t \mathrm{~d} x\right)^{\frac{1}{q^{\prime}}} \\
& \quad=C_{6} \lim _{\varepsilon \rightarrow 0}\left(\int_{\mathbb{R}} \varepsilon^{\frac{1}{2}}\left(\int_{\mathbb{R}^{3}}|f|^{q} \mathrm{~d} x\right) \mathrm{e}^{-\varepsilon t^{2}} \mathrm{~d} t\right)^{\frac{1}{q}}\left(\int_{\mathbb{R}} \varepsilon^{\frac{1}{2}}\left(\int_{\mathbb{R}^{3}}|g|^{q^{\prime}} \mathrm{d} x\right) \mathrm{e}^{-\varepsilon t^{2}} \mathrm{~d} t\right)^{\frac{1}{q^{\prime}}} .
\end{aligned}
$$

We can now conclude (3.38) from Lemma 3.4.4. 


\subsection{Time averaging}

Recall the decomposition described in Section 1.5 of a time-periodic function $u$ into a steady-state and a time-periodic part. Formally, the decomposition is given in (1.12) by simply computing the time average of $u$. Here, we shall define a similar time-averaging operator in the setting of vector fields defined on the group $G:=\mathbb{R}^{3} \times \mathbb{R} / \mathcal{T} \mathbb{Z}$.

Definition 3.5.1. We let

$$
\begin{aligned}
& \mathcal{P}: C_{0}^{\infty}(G) \rightarrow C_{0}^{\infty}(G), \quad \mathcal{P} f(x):=\frac{1}{\mathcal{T}} \int_{0}^{\mathcal{T}} f(x, t) \mathrm{d} t, \\
& \mathcal{P}_{\perp}: C_{0}^{\infty}(G) \rightarrow C_{0}^{\infty}(G), \quad \mathcal{P}_{\perp}:=\mathrm{Id}-\mathcal{P} .
\end{aligned}
$$

Both $\mathcal{P}$ and $\mathcal{P}_{\perp}$ extend naturally to the space vector fields $C_{0}^{\infty}(G)^{3}$. We collect the basic properties of $\mathcal{P}$ and $\mathcal{P}_{\perp}$ in the following lemma.

Lemma 3.5.2. Let $q \in(1, \infty)$. $\mathcal{P}$ extends uniquely to a continuous projection

$$
\mathcal{P}: L^{q}(G) \rightarrow L^{q}(G), \quad \mathcal{P}: W^{1,2, q}(G) \rightarrow W^{1,2, q}(G) .
$$

Restricted to solenoidal fields, $\mathcal{P}$ extends uniquely to a continuous projection

$$
\mathcal{P}: L_{\sigma}^{q}(G) \rightarrow L_{\sigma}^{q}(G), \quad \mathcal{P}: W_{\sigma}^{1,2, q}(G) \rightarrow W_{\sigma}^{1,2, q}(G) .
$$

The same statements are true for $\mathcal{P}_{\perp}$.

Proof. Clearly, $\mathcal{P}$ is a projection, that is, $\mathcal{P}^{2}=\mathcal{P}$. Moreover, employing first Minkowski's integral inequality and then Hölder's inequality we find that

$$
\begin{aligned}
\|\mathcal{P} f\|_{L^{q}(G)} & =\left(\frac{1}{\mathcal{T}} \int_{0}^{\mathcal{T}} \int_{\mathbb{R}^{3}}\left|\frac{1}{\mathcal{T}} \int_{0}^{\mathcal{T}} f(x, s) \mathrm{d} s\right|^{q} \mathrm{~d} x \mathrm{~d} t\right)^{1 / q} \\
& =\left(\int_{\mathbb{R}^{3}}\left|\frac{1}{\mathcal{T}} \int_{0}^{\mathcal{T}} f(x, s) \mathrm{d} s\right|^{q} \mathrm{~d} x\right)^{1 / q} \\
& \leq \frac{1}{\mathcal{T}} \int_{0}^{\mathcal{T}}\left(\int_{\mathbb{R}^{3}}|f(x, s)|^{q} \mathrm{~d} x\right)^{1 / q} \mathrm{~d} s \leq\|f\|_{L^{q}(G)} .
\end{aligned}
$$

Thus, by density $\mathcal{P}$ extends uniquely to a continuous projection $\mathcal{P}: L^{q}(G) \rightarrow L^{q}(G)$. Estimating derivatives of $\mathcal{P} f$ in same manner, we find that $\mathcal{P}$ is also bounded with respect to the $W^{1,2, q}(G)$-norm. Consequently, $\mathcal{P}$ extends uniquely to a continuous projection $\mathcal{P}: W^{1,2, q}(G) \rightarrow W^{1,2, q}(G)$. Finally, since $\operatorname{div} \mathcal{P} f=\mathcal{P} \operatorname{div} f$ for any $f \in C_{0}^{\infty}(G)^{3}$, we have $\mathcal{P}: C_{0, \sigma}^{\infty}(G) \rightarrow C_{0, \sigma}^{\infty}(G)$. Employing the density properties from Lemma 3.2.1, we deduce (3.40). Since (3.39) and (3.40) hold for $\mathcal{P}$, it follows trivially that the same is true for $\mathcal{P}_{\perp}$. 


\section{Linear theory}

As described in Section 1.5, we shall use $\mathcal{P}$ and $\mathcal{P}_{\perp}$ to decompose $L^{q}(G)$ and $W^{1,2, q}(G)$ into direct sums of functions that are time-independent, i.e., steady states, and functions that have vanishing time-average.

Lemma 3.5.3. Let $q \in(1, \infty)$. The projection $\mathcal{P}$ induces the decompositions

$$
\begin{aligned}
& L^{q}(G)=L^{q}\left(\mathbb{R}^{3}\right) \oplus L_{\perp}^{q}(G), \\
& L_{\sigma}^{q}(G)=L_{\sigma}^{q}\left(\mathbb{R}^{3}\right) \oplus L_{\sigma, \perp}^{q}(G), \\
& W_{\sigma}^{1,2, q}(G)=W_{\sigma}^{2, q}\left(\mathbb{R}^{3}\right) \oplus W_{\sigma, \perp}^{1,2, q}(G)
\end{aligned}
$$

with

$$
\begin{aligned}
& L_{\perp}^{q}(G):=\mathcal{P}_{\perp} L^{q}(G), \\
& L_{\sigma, \perp}^{q}(G):=\mathcal{P}_{\perp} L_{\sigma}^{q}(G), \\
& W_{\sigma, \perp}^{1,2, q}(G):=\mathcal{P}_{\perp} W_{\sigma}^{1,2, q}(G) .
\end{aligned}
$$

Proof. Since, by Lemma 3.5.2, $\mathcal{P}: L^{q}(G) \rightarrow L^{q}(G)$ is a continuous projection, it follows that

$$
L^{q}(G)=\mathcal{P} L^{q}(G) \oplus \mathcal{P}_{\perp} L^{q}(G) .
$$

Consequently, to show (3.41) we need only to verify that $\mathcal{P} L^{q}(G)=L^{q}\left(\mathbb{R}^{3}\right)$. This, however, is an easy consequence of the fact that $\mathcal{P} f$ is independent on $t$ and thus $\|\mathcal{P} f\|_{L^{q}\left(\mathbb{R}^{3}\right)}=\|\mathcal{P} f\|_{L^{q}(G)}$. The decompositions (3.42) and (3.43) follow analogously.

It will be convenient to extend the projections $\mathcal{P}$ and $\mathcal{P}_{\perp}$ to the space $L_{l o c}^{1}(G)$.

Lemma 3.5.4. $\mathcal{P}$ and $\mathcal{P}_{\perp}$ extend uniquely to projections

$$
\mathcal{P}: L_{l o c}^{1}(G) \rightarrow L_{l o c}^{1}(G), \quad \mathcal{P}_{\perp}: L_{l o c}^{1}(G) \rightarrow L_{l o c}^{1}(G) .
$$

Proof. The projection $\mathcal{P}: C_{0}^{\infty}(G) \rightarrow C_{0}^{\infty}(G)$ extends naturally to elements $u \in L_{l o c}^{1}(G)$ by defining $\mathcal{P} u$ in $L^{1}\left(\mathrm{~B}_{R} \times \mathbb{R} / \mathcal{T} \mathbb{Z}\right)$ for any $R>0$. Then $\mathcal{P} u$ and thus also $\mathcal{P}_{\perp} u$ are well-defined as elements in $L_{l o c}^{1}(G)$. Clearly, the extensions (3.47) are projections.

Lemma 3.5.5. Let $f, g \in L_{l o c}^{1}(G)$. Then

$$
\frac{1}{\mathcal{T}} \int_{0}^{\mathcal{T}} \mathcal{P} f(x, t) \cdot \mathcal{P}_{\perp} g(x, t) \mathrm{d} t=0 \quad \text { for a.e. } x \in \mathbb{R}^{3} .
$$

Proof. Since $\mathcal{P} f$ is independent on $t$, we have

$$
\frac{1}{\mathcal{T}} \int_{0}^{\mathcal{T}} \mathcal{P} f(x, t) \cdot \mathcal{P}_{\perp} g(x, t) \mathrm{d} t=\mathcal{P} f(x) \cdot \frac{1}{\mathcal{T}} \int_{0}^{\mathcal{T}}[\mathrm{Id}-\mathcal{P}] g(x, t) \mathrm{d} t=0
$$

for almost every $x \in \mathbb{R}^{3}$. 
Next, we compute the symbols of $\mathcal{P}$ and $\mathcal{P}_{\perp}$ with respect to the Fourier transform on $G$. These symbols will play a very important role later on, so even though the computation is trivial we state the result in a lemma.

Lemma 3.5.6. For $f \in \mathscr{S}(G)$

$$
\begin{aligned}
& \mathcal{P} f=\mathcal{F}_{G}^{-1}\left[\kappa_{0} \cdot \widehat{f}\right], \\
& \mathcal{P}_{\perp} f=\mathcal{F}_{G}^{-1}\left[\left(1-\kappa_{0}\right) \cdot \widehat{f}\right]
\end{aligned}
$$

with

$$
\kappa_{0}: \widehat{G} \rightarrow \mathbb{C}, \quad \kappa_{0}(\xi, k):= \begin{cases}1 & \text { if } k=0, \\ 0 & \text { if } k \neq 0 .\end{cases}
$$

Proof. We simply observe that

$$
\begin{aligned}
\mathcal{F}[\mathcal{P} f](\xi, k) & =\frac{1}{\mathcal{T}} \int_{0}^{\mathcal{T}} \int_{\mathbb{R}^{3}} \frac{1}{\mathcal{T}} \int_{0}^{\mathcal{T}} f(x, s) \mathrm{d} s e^{-i x \cdot \xi-i \frac{2 \pi}{\mathcal{T}} k t} \mathrm{~d} x \mathrm{~d} t \\
& =\kappa_{0}(\xi, k) \int_{\mathbb{R}^{3}} \frac{1}{\mathcal{T}} \int_{0}^{\mathcal{T}} f(x, s) \mathrm{d} s e^{-i x \cdot \xi} \mathrm{d} x \\
& =\kappa_{0}(\xi, k) \widehat{f}(\xi, 0)=\kappa_{0}(\xi, k) \widehat{f}(\xi, k) .
\end{aligned}
$$

As one may recall, the compact-open topology of $\widehat{G}=\mathbb{R}^{3} \times \mathbb{Z}$ simply reduces to the product of the Euclidean topology of $\mathbb{R}^{3}$ and the discrete topology of $\mathbb{Z}$. Thus $\kappa_{0}: \widehat{G} \rightarrow \mathbb{C}$ is in fact a continuous and bounded Fourier multiplier. Moreover, $\mathscr{S}^{\prime}(\widehat{G})$ is clearly closed under multiplication by $\kappa_{0}$. Thus, both (3.49) and (3.50) can be extended to the case $f \in \mathscr{S}^{\prime}(G)$.

Definition 3.5.7. For $f \in \mathscr{S}^{\prime}(G)$ we define

$$
\mathcal{P} f:=\mathcal{F}_{G}^{-1}\left[\kappa_{0} \cdot \widehat{f}\right], \quad \mathcal{P}_{\perp} f:=\mathcal{F}_{G}^{-1}\left[\left(1-\kappa_{0}\right) \cdot \widehat{f}\right] .
$$

As another trivial consequence of Lemma 3.5.6, we see that:

Corollary 3.5.8. $\mathcal{P}$ commutes with the Helmholtz projection $\mathcal{P}_{H}$ from Lemma 3.2.3.

\subsection{Maximal regularity}

We are now in a position to identify the function spaces that establish maximal regularity for the linearized time-periodic Navier-Stokes system (3.1). We recall the formulation

$$
\begin{cases}\partial_{t} u-\Delta u-\lambda \partial_{1} u+\nabla \mathfrak{p}=f & \text { in } G, \\ \operatorname{div} u=0 & \text { in } G\end{cases}
$$




\section{Linear theory}

of (3.1) in a setting of vector fields defined on $G$. Here we shall study the resolvability of (3.51) for arbitrary $f \in L^{q}(G)^{3}$.

For simplicity, the condition

$$
\lim _{|x| \rightarrow \infty} u(x, t)=0
$$

has been omitted in (3.51). We will investigate the system in a setting with $u$ belonging to a subspace of $L^{q}(G)$ and thus automatically vanish at spatial infinity in the sense that it is $L^{q}$-summable over $\mathbb{R}^{3}$.

In the first step, we utilize the Helmholtz decomposition to eliminate the pressure term in (3.51). More specifically, by applying $\mathcal{P}_{H}$ to both sides in (3.51) we reduce the investigation of (3.51) to the investigation of

$$
\begin{cases}\partial_{t} u-\Delta u-\lambda \partial_{1} u=\mathcal{P}_{H} f & \text { in } G, \\ \operatorname{div} u=0 & \text { in } G .\end{cases}
$$

The pressure term $\mathfrak{p}$ can be recovered as $\left[\mathrm{Id}-\mathcal{P}_{H}\right] f$.

One of the key ideas used in the following is to utilize the decomposition (3.41) to split (3.53) into two separate systems. More precisely, we use the projection $\mathcal{P}$ to express $\mathcal{P}_{H} f$ as

$$
\mathcal{P}_{H} f=\mathcal{P} \mathcal{P}_{H} f+\mathcal{P}_{\perp} \mathcal{P}_{H} f \in L_{\sigma}^{q}\left(\mathbb{R}^{3}\right) \oplus L_{\sigma, \perp}^{q}(G),
$$

and seek a solution $u$ to (3.53) as a sum

$$
u=v+w
$$

of a solution $v$ to

$$
\begin{cases}-\Delta v-\lambda \partial_{1} v=\mathcal{P} \mathcal{P}_{H} f & \text { in } \mathbb{R}^{3} \\ \operatorname{div} v=0 & \text { in } \mathbb{R}^{3}\end{cases}
$$

and a solution $w$ to

$$
\begin{cases}\partial_{t} w-\Delta w-\lambda \partial_{1} w=\mathcal{P}_{\perp} \mathcal{P}_{H} f & \text { in } G, \\ \operatorname{div} w=0 & \text { in } G .\end{cases}
$$

Recall that $\mathcal{P} \mathcal{P}_{H} f$ is $t$-independent, whence (3.55) reduces to a steady-state problem. We recognize $(3.55)$ as the steady-state Oseen $(\lambda \neq 0)$ or steady-state Stokes $(\lambda=0)$ system in the whole space.

We will study (3.53), (3.55), and (3.56) in a setting of linear operators. We therefore put

$$
\mathrm{A}_{T P}: \mathscr{S}^{\prime}(G)^{3} \rightarrow \mathscr{S}^{\prime}(G)^{3}, \quad \mathrm{~A}_{T P} u:=\partial_{t} u-\Delta u-\lambda \partial_{1} u .
$$


As will be clear shortly, in the study of (3.55) it will be necessary to treat the Oseen case $(\lambda \neq 0)$ and Stokes case $(\lambda=0)$ separately. We therefore define for $\lambda \neq 0$

$$
A_{\text {Oseen }}: \mathscr{S}^{\prime}\left(\mathbb{R}^{3}\right)^{3} \rightarrow \mathscr{S}^{\prime}\left(\mathbb{R}^{3}\right)^{3}, \quad A_{\text {Oseen }} v:=-\Delta v-\lambda \partial_{1} v,
$$

and let

$$
A_{\text {Stokes }}: \mathscr{S}^{\prime}\left(\mathbb{R}^{3}\right)^{3} \rightarrow \mathscr{S}^{\prime}\left(\mathbb{R}^{3}\right)^{3}, \quad A_{\text {Stokes }} v:=-\Delta v .
$$

Restricted to a setting of solenoidal vector fields, $A_{\text {Oseen }}$ is known as the Oseen operator, and $A_{\text {Stokes }}$ as the Stokes operator. ${ }^{2}$

In the next step, we shall identify subspaces $\mathrm{X}_{\sigma, \text { Oseen }}^{q}\left(\mathbb{R}^{3}\right)$ and $\mathrm{X}_{\sigma, \text { Stokes }}^{q}\left(\mathbb{R}^{3}\right)$ of $L_{\sigma}^{q}\left(\mathbb{R}^{3}\right)$, and $\mathrm{X}_{\sigma, \perp}^{q}(G)$ of $L_{\sigma}^{q}(G)$ such that

$$
\begin{aligned}
& A_{\text {Oseen }}: \mathrm{X}_{\sigma, \text { Oseen }}^{q}\left(\mathbb{R}^{3}\right) \rightarrow L_{\sigma}^{q}\left(\mathbb{R}^{3}\right), \\
& A_{\text {Stokes }}: \mathrm{X}_{\sigma, \text { Stokes }}^{q}\left(\mathbb{R}^{3}\right) \rightarrow L_{\sigma}^{q}\left(\mathbb{R}^{3}\right), \\
& \mathrm{A}_{T P}: \mathrm{X}_{\sigma, \perp}^{q}(G) \rightarrow L_{\sigma, \perp}^{q}(G)
\end{aligned}
$$

are homeomorphisms. The identification of these function space is the cornerstone in the maximal regularity result for the time-periodic linearized Navier-Stokes system.

The properties of the Oseen and Stokes operators are well-known. For the Oseen operator we have:

Theorem 3.6.1. For $q \in(1,2)$ and $\lambda>0$ define

$$
\begin{aligned}
& \mathrm{X}_{\sigma, \text { Oseen }}^{q}\left(\mathbb{R}^{3}\right):=\left\{v \in L_{l o c}^{1}\left(\mathbb{R}^{3}\right)^{3} \mid \operatorname{div} v=0,\|v\|_{q, \lambda, \text { Oseen }}<\infty\right\}, \\
& \|v\|_{q, \lambda, \text { Oseen }}:=\lambda^{\frac{1}{2}}\|v\|_{\frac{2 q}{2-q}}+\lambda^{\frac{1}{4}}\|\nabla v\|_{\frac{4 q}{4-q}}+\lambda\left\|\partial_{1} v\right\|_{q}+\left\|\nabla^{2} v\right\|_{q} .
\end{aligned}
$$

Then $\mathrm{X}_{\sigma, \text { Oseen }}^{q}\left(\mathbb{R}^{3}\right)$ is a Banach space and

$$
A_{\text {Oseen }}: \mathrm{X}_{\sigma, \text { Oseen }}^{q}\left(\mathbb{R}^{3}\right) \rightarrow L_{\sigma}^{q}\left(\mathbb{R}^{3}\right)
$$

a homeomorphism. Moreover

$$
\left\|A_{\text {Oseen }}^{-1}\right\| \leq C_{7}
$$

with $C_{7}$ independent on $\lambda$.

Proof. See [17, Theorem VII.4.1].

In addition, we recall the following well-known property:

Lemma 3.6.2. Let $v \in L^{q}\left(\mathbb{R}^{3}\right)$ for some $q \in[1, \infty)$. If

$$
-\Delta v-\lambda \partial_{1} v=0 \quad \text { in } \mathbb{R}^{3},
$$

then $v=0$.

\footnotetext{
${ }^{2}$ Usually, the restriction of the Stokes and Oseen operator to a subspace of solenoidal vector fields is achieved by defining $A_{\text {Stokes }}:=-\mathcal{P}_{H} \Delta$ and $A_{\text {Oseen }}:=\mathcal{P}_{H}\left(-\Delta-\lambda \partial_{1}\right)$.
} 


\section{Linear theory}

Proof. Applying the Fourier transform $\mathcal{F}_{\mathbb{R}^{3}}$ on both sides in (3.58), we see that

$$
\left(|\xi|^{2}-\lambda i \xi_{1}\right) \widehat{v}=0
$$

It follows that $\operatorname{supp} \widehat{v} \subset\{0\}$, whence $v$ is a polynomial. Since $v \in L^{q}\left(\mathbb{R}^{3}\right)^{3}$, we must have $v=0$.

The Stokes operator enjoys the following mapping properties:

Theorem 3.6.3. For $q \in(1,3 / 2)$ define

$$
\begin{aligned}
& \mathrm{X}_{\sigma, \text { Stokes }}^{q}\left(\mathbb{R}^{3}\right):=\left\{v \in L_{l o c}^{1}\left(\mathbb{R}^{3}\right)^{3} \mid \operatorname{div} v=0,\|v\|_{q, \text { Stokes }}<\infty\right\}, \\
& \|v\|_{q, \text { Stokes }}:=\|v\|_{\frac{3 q}{3-2 q}}+\|\nabla v\|_{\frac{3 q}{3-q}}+\left\|\nabla^{2} v\right\|_{q} .
\end{aligned}
$$

Then $\mathrm{X}_{\sigma, \text { Stokes }}^{q}\left(\mathbb{R}^{3}\right)$ is a Banach space and

$$
A_{\text {Stokes }}: \mathrm{X}_{\sigma, \text { Stokes }}^{q}\left(\mathbb{R}^{3}\right) \rightarrow L_{\sigma}^{q}\left(\mathbb{R}^{3}\right)
$$

a homeomorphism.

Proof. See [17, Theorem IV.2.1].

The main challenge is now to characterize the space $\mathrm{X}_{\sigma, \perp}^{q}(G)$. We start with the following lemma.

Lemma 3.6.4. If $u \in \mathscr{S}^{\prime}(G)$ with $\mathcal{P} u=0$ satisfies

$$
\partial_{t} u-\Delta u-\lambda \partial_{1} u=0 \quad \text { in } G
$$

then $u=0$.

Proof. Applying the Fourier transform $\mathcal{F}_{G}$ on both sides in (3.59), we deduce

$$
\left(i \frac{2 \pi}{\mathcal{T}} k+|\xi|^{2}-\lambda i \xi_{1}\right) \widehat{u}=0
$$

Since

$$
|\xi|^{2}+i\left(\frac{2 \pi}{\mathcal{T}} k-\lambda \xi_{1}\right)=0 \quad \Longleftrightarrow \quad(\xi, k)=(0,0)
$$

we conclude that supp $\widehat{u} \subset\{(0,0)\}$. However, since $\mathcal{P} u=0$ we have $\kappa_{0} \widehat{u}=0$, whence $(\xi, 0) \notin \operatorname{supp} \widehat{u}$ for all $\xi \in \mathbb{R}^{3}$. Consequently, supp $\widehat{u}=\emptyset$. It follows that $\widehat{u}=0$ and thus $u=0$.

In the following theorem, we finally characterize the space $\mathrm{X}_{\sigma, \perp}^{q}(G)$. 
Theorem 3.6.5. Let $q \in(1, \infty)$. Then

$$
\mathrm{A}_{T P}: W_{\sigma, \perp}^{1,2, q}(G) \rightarrow L_{\sigma, \perp}^{q}(G)
$$

homeomorphically. Moreover

$$
\left\|A_{\mathrm{TP}}^{-1}\right\| \leq C_{8} P(\lambda, \mathcal{T}),
$$

where $C_{8}=C_{8}(q)$ and $P(\lambda, \mathcal{T})$ is a polynomial in $\lambda$ and $\mathcal{T}$.

Proof. By construction, $\mathrm{A}_{T P}$ maps $W_{\sigma}^{1,2, q}(G)$ into $L_{\sigma}^{q}(G)$. Clearly

$$
\mathrm{A}_{T P}: W_{\sigma}^{1,2, q}(G) \rightarrow L_{\sigma}^{q}(G)
$$

is bounded. Moreover, as one may easily verify, the diagram

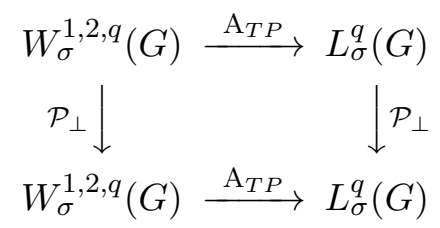

commutes. Thus also

$$
\mathrm{A}_{T P}: W_{\sigma, \perp}^{1,2, q}(G) \rightarrow L_{\sigma, \perp}^{q}(G)
$$

is a bounded map.

We shall now show that the mapping (3.61) is onto. To this end, consider first some $f \in \mathcal{P}_{\perp} C_{0, \sigma}^{\infty}(G)$. Clearly, $f \in \mathscr{S}(G)^{3}$. In view of (3.28), we put

$$
u:=\mathcal{F}_{G}^{-1}\left[\frac{1}{i \frac{2 \pi}{\mathcal{T}} k+|\xi|^{2}-\lambda i \xi_{1}} \widehat{f}\right] .
$$

At the outset, $u$ is well-defined as an element of $\mathscr{S}^{\prime}(G)^{3}$. It is easy to verify that

$$
\mathrm{A}_{T P} u=f .
$$

The challenge is now to show that $u \in W_{\sigma, \perp}^{1,2, q}(G)$ and establish the estimate

$$
\|u\|_{1,2, q} \leq c\|f\|_{q}
$$

for some constant $c$. Since $f=\mathcal{P}_{\perp} f$, we recall (3.50) and deduce

$$
u=\mathcal{F}_{G}^{-1}\left[\frac{\left(1-\kappa_{0}(\xi, k)\right)}{i \frac{2 \pi}{\mathcal{T}} k+|\xi|^{2}-\lambda i \xi_{1}} \widehat{f}\right] .
$$

For convenience, we put

$$
M: \widehat{G} \rightarrow \mathbb{C}, \quad M(\xi, k):=\frac{1-\kappa_{0}(\xi, k)}{|\xi|^{2}+i\left(\frac{2 \pi}{\mathcal{T}} k-\lambda \xi_{1}\right)}
$$




\section{Linear theory}

and write

$$
u=\mathcal{F}_{G}^{-1}[M(\xi, k) \cdot \widehat{f}] .
$$

We shall use the transference principle for multipliers, that is, Theorem 3.4.1, to establish (3.62). For this purpose, let $\chi$ be a "cut-off" function with

$$
\chi \in C_{0}^{\infty}(\mathbb{R} ; \mathbb{R}), \quad \chi(\eta)=1 \text { for }|\eta| \leq \frac{1}{2}, \quad \chi(\eta)=0 \text { for }|\eta| \geq 1 .
$$

We then define

$$
m: \mathbb{R}^{3} \times \mathbb{R} \rightarrow \mathbb{C}, \quad m(\xi, \eta):=\frac{1-\chi\left(\frac{\mathcal{T}}{2 \pi} \eta\right)}{|\xi|^{2}+i\left(\eta-\lambda \xi_{1}\right)} .
$$

We can consider $\mathbb{R}^{3} \times \mathbb{R}$ as a group $H$ with addition as group operation. Endowed with the Euclidean topological, $H$ becomes a locally compact abelian group. It is well-known that the dual group $\widehat{H}$ can also be identified with $\mathbb{R}^{3} \times \mathbb{R}$ equipped with the Euclidean topology. We can thus consider $m$ as mapping

$$
m: \widehat{H} \rightarrow \mathbb{C} .
$$

In order to employ Theorem 3.4.1, we let

$$
\Phi: \widehat{G} \rightarrow \widehat{H}, \quad \Phi(\xi, k)=\left(\xi, \frac{2 \pi}{\mathcal{T}} k\right) .
$$

Clearly, $\Phi$ is a continuous homomorphism. Moreover,

$$
M=m \circ \Phi .
$$

Consequently, if we can show that $m$ is a continuous $L^{q}\left(\mathbb{R}^{3} \times \mathbb{R}\right)$-multiplier we may conclude from Theorem 3.4.1, or Theorem 3.4.5, that $M$ is a $L^{q}(G)$-multiplier. To see that $m$ is a continuous $L^{q}$-multiplier, we recall that

$$
|\xi|^{2}+i\left(\eta-\lambda \xi_{1}\right)=0 \quad \Longleftrightarrow \quad(\xi, \eta)=(0,0) .
$$

Thus, the only zero of the denumerator in $(3.66)$ is $(0,0)$. Since the numerator $(\xi, \eta) \rightarrow$ $1-\chi\left(\frac{\mathcal{T}}{2 \pi} \eta\right)$ in (3.66) vanishes in a neighborhood of $(0,0)$, however, we see that $m$ is continuous; in fact $m$ is smooth. We shall now apply Marcinkiewicz's multiplier theorem to show that $m$ is an $L^{q}\left(\mathbb{R}^{3} \times \mathbb{R}\right)$-multiplier. Note that Marcinkiewicz's multiplier theorem can be employed at this point since $m$ is a Fourier multiplier in an $\mathbb{R}^{n}$ setting ( $\mathbb{R}^{4}$ to be precise). We must show, recall Theorem 2.4.1, that

$$
\sup _{\varepsilon \in\{0,1\}^{4}} \sup _{(\xi, \eta) \in \mathbb{R}^{3} \times \mathbb{R}}\left|\xi_{1}^{\varepsilon_{1}} \xi_{2}^{\varepsilon_{2}} \xi_{3}^{\varepsilon_{3}} \eta^{\varepsilon_{4}} \partial_{1}^{\varepsilon_{1}} \partial_{2}^{\varepsilon_{2}} \partial_{3}^{\varepsilon_{3}} \partial_{\eta}^{\varepsilon_{4}} m(\xi, \eta)\right| \leq c_{1} .
$$

Given the structure of $m$, this is in fact quite easy to verify. Since $m$ is smooth, we only need to show that all functions of type

$$
(\xi, \eta) \rightarrow \xi_{1}^{\varepsilon_{1}} \xi_{2}^{\varepsilon_{2}} \xi_{3}^{\varepsilon_{3}} \eta^{\varepsilon_{4}} \partial_{1}^{\varepsilon_{1}} \partial_{2}^{\varepsilon_{2}} \partial_{3}^{\varepsilon_{3}} \partial_{\eta}^{\varepsilon_{4}} m(\xi, \eta) .
$$


stay bounded as $|(\xi, \eta)| \rightarrow \infty$. As these functions are rational functions away from $(0,0)$, this is easy to verify. Consequently, we conclude (3.70). If we analyze the bound on the functions more carefully, we find that $c_{1}$ can be chosen such that

$$
c_{1}=P_{1}(\lambda, \mathcal{T}),
$$

with $P_{1}(\lambda, \mathcal{T})$ a polynomial in $\lambda$ and $\mathcal{T}$. By Theorem 2.4.1, $m$ is an $L^{q}\left(\mathbb{R}^{4}\right)$-multiplier. In turn, due to (3.68) it follows from Theorem 3.4.1, or Theorem 3.4.5, that $M$ is an $L^{q}(G)$-multiplier. Recalling (3.65), we thus obtain

$$
\|u\|_{q} \leq c_{2} P_{1}(\lambda, \mathcal{T})\|f\|_{q},
$$

with $c_{2}=c_{2}(q)$. Differentiating $u$ with respect to time and space, we obtain from (3.65) the representation formulas

$$
\partial_{t} u=\mathcal{F}_{G}^{-1}\left[\left(i \frac{2 \pi}{\mathcal{T}} k\right) M(\xi, k) \cdot \widehat{f}\right]
$$

and

$$
\partial_{x}^{\alpha} u=\mathcal{F}_{G}^{-1}\left[(i \xi)^{\alpha} M(\xi, k) \cdot \widehat{f}\right],
$$

respectively. We can now repeat the argument above with $\left(i \frac{2 \pi}{\mathcal{T}} k\right) M(\xi, k)$ in the role of the multiplier $M$, and $\left(i \frac{2 \pi}{\mathcal{T}} \eta\right) m(\xi, \eta)$ in the role of $m$, to conclude

$$
\left\|\partial_{t} u\right\|_{q} \leq c_{3} P_{2}(\lambda, \mathcal{T})\|f\|_{q},
$$

with $c_{3}=c_{3}(q)$. Similarly, for $|\alpha| \leq 2$ we repeat the argument above with $(i \xi)^{\alpha} M(\xi, k)$ in the role of $M$, and $(i \xi)^{\alpha} m(\xi, \eta)$ in the role of $m$, and obtain

$$
\left\|\partial_{x}^{\alpha} u\right\|_{q} \leq c_{4} P_{3}(\lambda, \mathcal{T})\|f\|_{q} .
$$

Collecting (3.72), (3.73), and (3.74), we thus conclude

$$
\|u\|_{1,2, q} \leq c_{5} P_{4}(\lambda, \mathcal{T})\|f\|_{q},
$$

with $c_{5}=c_{5}(q)$. Recalling (2.6), (3.50), and the fact that $\mathcal{P}_{H} f=f$ and $\mathcal{P}_{\perp} f=f$, we see directly from (3.63) that also $\mathcal{P}_{H} u=u$ and $\mathcal{P}_{\perp} u=u$. Thus $u \in W_{\sigma \perp}^{1,2, q}(G)$. Consequently, we have constructed for arbitrary $f \in \mathcal{P}_{\perp} C_{0, \sigma}^{\infty}(G)$ a vector field $u \in$ $W^{1,2, q}(G)$ such that $\mathrm{A}_{T P} u=f$ and for which (3.75) holds. Since $C_{0, \sigma}^{\infty}(G)$ is a dense subset of $L_{\sigma}^{q}(G)$, recall Lemma 3.2.1, it follows that $\mathcal{P}_{\perp} C_{0, \sigma}^{\infty}(G)$ is dense in $L_{\sigma, \perp}^{q}(G)$. Thus, by a standard density argument, we can find for any $f \in L_{\sigma, \perp}^{q}(G)$ a vector field $u \in W_{\sigma, \perp}^{1,2, q}(G)$ that satisfies $\mathrm{A}_{T P} u=f$ and satisfies (3.75). In particular, we have verified that the map (3.61) is onto.

Finally, we must verify that the map in (3.61) is injective. This, however, follows directly from Lemma 3.6.4.

Since the map in (3.61) is bounded and bijective, it is a homeomorphism by the open mapping theorem. The bound (3.60) follows from (3.75). 


\section{Linear theory}

Remark 3.6.6. The key reason the argument above works is that the numerator $1-\chi$ in the fraction that defines the multiplier $m$ in (3.66) vanishes in a neighborhood of the only zero of the denumerator

$$
(\xi, \eta) \rightarrow|\xi|^{2}+i\left(\eta-\lambda \xi_{1}\right)
$$

As a consequence, $m$ is bounded and smooth. If we did not have $1-\chi$ in the numerator of $m$, that is, if the multiplier under investigation was

$$
\widetilde{m}: \mathbb{R}^{3} \times \mathbb{R} \rightarrow \mathbb{C}, \quad \widetilde{m}(\xi, \eta):=\frac{1}{|\xi|^{2}+i\left(\eta-\lambda \xi_{1}\right)},
$$

the multiplier would have a singularity at $(\xi, \eta)=(0,0)$. It is easy the see that $\widetilde{m}$ is not an $L^{q}$-multiplier. The appearance of $1-\chi$ in the numerator of $m$ is therefore crucial to the proof of Theorem 3.6.5. The reason we can put $1-\chi$ in the numerator of $m$ is due to the restriction of $\mathrm{A}_{T P}$ to the subspace $W_{\sigma, \perp}^{1,2, q}(G)=\mathcal{P}_{\perp} W_{\sigma}^{1,2, q}(G)$. More precisely, it is a consequence of $1-\kappa_{0}$ being the Fourier multiplier of $\mathcal{P}_{\perp}$. This observation illustrates the importance of the decomposition of the function spaces induced by the projections $\mathcal{P}$ and $\mathcal{P}_{\perp}$.

The combination of Theorem 3.6.1 and Theorem 3.6.5 establish maximal regularity of the time-periodic linearized Navier-Stokes in the case $\lambda \neq 0$. Similarly, the combination of Theorem 3.6.3 and Theorem 3.6.5 establish maximal regularity in the case $\lambda=0$. We manifest this in the following theorem:

Theorem 3.6.7. If $q \in(1,2)$ and $\lambda>0$, then

$$
\begin{aligned}
& A_{\lambda, \sigma}: \mathrm{X}_{\sigma, \text { Oseen }}^{q}\left(\mathbb{R}^{3}\right) \times W_{\sigma, \perp}^{1,2, q}(G) \rightarrow L_{\sigma}^{q}(G), \\
& A_{\lambda, \sigma}(v, w):=\partial_{t} w-\Delta(v+w)-\lambda \partial_{1}(v+w)
\end{aligned}
$$

is a homeomorphism and

$$
\left\|A_{\lambda, \sigma}^{-1}\right\| \leq C_{9} P(\lambda, \mathcal{T})
$$

where $C_{9}=C_{9}(q)$ and $P(\lambda, \mathcal{T})$ is a polynomial in $\lambda$ and $\mathcal{T}$.

If $q \in\left(1, \frac{3}{2}\right)$, then

$$
\begin{aligned}
& A_{\sigma}: \mathrm{X}_{\sigma, \text { Stokes }}^{q}\left(\mathbb{R}^{3}\right) \times W_{\sigma, \perp}^{1,2, q}(G) \rightarrow L_{\sigma}^{q}(G), \\
& A_{\sigma}(v, w):=\partial_{t} w-\Delta(v+w)
\end{aligned}
$$

is a homeomorphism and

$$
\left\|A_{\sigma}^{-1}\right\| \leq C_{10} P(\lambda, \mathcal{T})
$$

where $C_{10}=C_{10}(q)$ and $P(\lambda, \mathcal{T})$ is a polynomial in $\lambda$ and $\mathcal{T}$. 
Proof. Consider first $q \in(1,2)$ and $\lambda>0$. Clearly, $A_{\lambda, \sigma}$ is a bounded linear mapping from $\mathrm{X}_{\sigma, \text { Oseen }}^{q}\left(\mathbb{R}^{3}\right) \times W_{\sigma, \perp}^{1,2, q}(G)$ into $L_{\sigma}^{q}(G)$. To see that $A_{\lambda, \sigma}$ is injective, consider an element $(v, w) \in \operatorname{ker} A_{\lambda, \sigma}$. Then

$$
\partial_{t} w-\Delta(v+w)-\lambda \partial_{1}(v+w)=0
$$

Applying the projection $\mathcal{P}$ to this identity yields $A_{\text {Oseen }} v=0$. Since, by Theorem 3.6.1, $A_{\text {Oseen }}: \mathrm{X}_{\sigma, \text { Oseen }}^{q}\left(\mathbb{R}^{3}\right) \rightarrow L_{\sigma}^{q}\left(\mathbb{R}^{3}\right)$ is a homeomorphism, we must have $v=0$. It then follows that $\mathrm{A}_{T P} w=0$, which, since by Theorem 3.6.5 also $\mathrm{A}_{T P}: W_{\sigma, \perp}^{1,2, q}(G) \rightarrow L_{\sigma, \perp}^{q}(G)$ is a homeomorphism, implies $w=0$. We conclude that $A_{\lambda, \sigma}$ is injective. To see that $A_{\lambda, \sigma}$ is onto, consider an element $f \in L_{\sigma}^{q}(G)$. We can decompose

$$
f=\mathcal{P} f+\mathcal{P}_{\perp} f \in L_{\sigma}^{q}\left(\mathbb{R}^{3}\right) \oplus L_{\sigma, \perp}^{q}(G)
$$

according to (3.42). Letting

$$
(v, w):=\left(A_{\text {Oseen }}^{-1}(\mathcal{P} f), A_{\mathrm{TP}}^{-1}\left(\mathcal{P}_{\perp} f\right)\right),
$$

we see that $A_{\lambda, \sigma}(v, w)=f$. Consequently, $A_{\lambda, \sigma}$ is onto. Moreover, since

$$
\begin{aligned}
\|v\|_{\mathrm{X}_{\sigma, \text { Oseen }}^{q}\left(\mathbb{R}^{3}\right)}+\|w\|_{W_{\sigma, \perp}^{1,2, q}(G)} & \leq\left\|A_{\text {Oseen }}^{-1}\right\|\|\mathcal{P} f\|_{q}+\left\|A_{\mathrm{TP}}^{-1}\right\|\left\|\mathcal{P}_{\perp} f\right\|_{q} \\
& \leq\left(\left\|A_{\text {Oseen }}^{-1}\right\|+\left\|A_{\mathrm{TP}}^{-1}\right\|\right)\|f\|_{q},
\end{aligned}
$$

we deduce from (3.57) and (3.60) that $A_{\lambda, \sigma}^{-1}$ is bounded and satisfies (3.77). This concludes the first part of the theorem. Employing Theorem 3.6.3 instead of Theorem 3.6.1, the second part can be shown by the same arguments.

Theorem 3.6.7 yields maximal regularity for the system (3.53) in a setting of solenoidal vector fields. To establish maximal regularity for the original system (3.51), we must include the pressure term $\mathfrak{p}$ in the function spaces. For this purpose, we need the following lemma:

Lemma 3.6.8. Let $q \in(1,3)$. Put

$$
\begin{aligned}
& \mathrm{X}_{\mathrm{P}}^{q}(G):=\left\{\mathfrak{p} \in L_{l o c}^{1}(G) \mid\|\mathfrak{p}\|_{\mathrm{X}_{\mathrm{P}}^{q}(G)}<\infty\right\}, \\
& \|\mathfrak{p}\|_{\mathrm{X}_{\mathrm{P}}^{q}(G)}:=\left(\frac{1}{\mathcal{T}} \int_{0}^{\mathcal{T}}\|\mathfrak{p}(\cdot, t)\|_{\frac{3 q}{3-q}}^{q} \mathrm{~d} t+\|\nabla \mathfrak{p}\|_{q}^{q}\right)^{1 / q} .
\end{aligned}
$$

Then

$$
\operatorname{grad}: \mathrm{X}_{\mathrm{P}}^{q}(G) \rightarrow \mathscr{G}^{q}(G), \quad \operatorname{grad} \mathfrak{p}:=\nabla \mathfrak{p}
$$

is a homeomorphism. 


\section{Linear theory}

Proof. Clearly, grad is a bounded linear map. Consider $\mathfrak{p} \in$ ker grad. Then $\nabla \mathfrak{p}=0$ in $G$ and thus $\mathfrak{p}(x, t)=c(t)$. Since $\|\mathfrak{p}\|_{\mathrm{X}_{\mathrm{P}}^{q}(G)}<\infty$, we must have $\mathfrak{p}=0$. Consequently, grad is injective. To show that grad is onto, we consider the mapping

$$
\mathcal{I}: \mathscr{S}(G)^{3} \rightarrow \mathscr{S}^{\prime}(G), \quad \mathcal{I}(f):=\mathcal{F}_{\mathbb{R}^{3}}^{-1}\left[\frac{\xi_{j}}{|\xi|^{2}} \cdot \mathcal{F}_{\mathbb{R}^{3}}\left[f_{j}\right]\right],
$$

where $\mathcal{F}_{\mathbb{R}^{3}}: \mathscr{S}(G) \rightarrow \mathscr{S}(G)$ denotes the partial Fourier transform. Observe that

$$
\nabla \mathcal{I}(f)=\left[\mathrm{Id}-\mathcal{P}_{H}\right] f .
$$

By well-known properties of the Riesz potential (see for example [29, Theorem 6.1.3]), we find that

$$
\frac{1}{\mathcal{T}} \int_{0}^{\mathcal{T}}\|\mathcal{I}(f)(\cdot, t)\|_{\frac{3 q}{3-q}}^{q} \mathrm{~d} t \leq c_{1} \frac{1}{\mathcal{T}} \int_{0}^{\mathcal{T}}\|f(\cdot, t)\|_{q}^{q} \mathrm{~d} t=c_{1}\|f\|_{q}^{q} .
$$

In combination with (3.82), this estimate yields

$$
\|\mathcal{I}(f)\|_{\mathrm{X}_{\mathrm{P}}^{q}(G)} \leq c_{2}\|f\|_{q} .
$$

By a density argument, we can extend $\mathcal{I}$ uniquely to a bounded map

$$
\mathcal{I}: L^{q}(G)^{3} \rightarrow \mathrm{X}_{\mathrm{P}}^{q}(G)
$$

that satisfies (3.82) for all $f \in L^{q}(G)^{3}$. We can now show that grad is onto. If namely $f \in \mathscr{G}^{q}(G)$, then $\nabla \mathcal{I}(f)=f$. We conclude by the open mapping theorem that grad is a homeomorphism. In fact, the inverse is given by $\mathcal{I}$.

Combining Theorem 3.6.7 and Lemma 3.6.8, we obtain:

Theorem 3.6.9. If $q \in(1,2)$ and $\lambda>0$, then

$$
\begin{aligned}
& A_{\lambda}: \mathrm{X}_{\sigma, \text { Oseen }}^{q}\left(\mathbb{R}^{3}\right) \times W_{\sigma, \perp}^{1,2, q}(G) \times \mathrm{X}_{\mathrm{P}}^{q}(G) \rightarrow L^{q}(G)^{3}, \\
& A_{\lambda}(v, w, \mathfrak{p}):=\partial_{t} w-\Delta(v+w)-\lambda \partial_{1}(v+w)+\nabla \mathfrak{p}
\end{aligned}
$$

is a homeomorphism and

$$
\left\|A_{\lambda}^{-1}\right\| \leq C_{11} P(\lambda, \mathcal{T})
$$

where $C_{11}=C_{11}(q)$ and $P(\lambda, \mathcal{T})$ is a polynomial in $\lambda$ and $\mathcal{T}$.

If $q \in\left(1, \frac{3}{2}\right)$, then

$$
\begin{aligned}
& A: \mathrm{X}_{\sigma, \text { Stokes }}^{q}\left(\mathbb{R}^{3}\right) \times W_{\sigma, \perp}^{1,2, q}(G) \times \mathrm{X}_{\mathrm{P}}^{q}(G) \rightarrow L^{q}(G)^{3}, \\
& A(v, w, \mathfrak{p}):=\partial_{t} w-\Delta(v+w)+\nabla \mathfrak{p}
\end{aligned}
$$

is a homeomorphism and

$$
\left\|A^{-1}\right\| \leq C_{12} P(\lambda, \mathcal{T})
$$

where $C_{12}=C_{12}(q)$ and $P(\lambda, \mathcal{T})$ is a polynomial in $\lambda$ and $\mathcal{T}$.

Proof. We simply recall from Lemma 2.3.4 the decomposition $L^{q}(G)=L_{\sigma}^{q}(G) \oplus \mathscr{G}^{q}(G)$ and combine Theorem 3.6.7 and Lemma 3.6.8. 


\subsection{Flow past a rotating body}

We end the chapter with an application of the maximal regularity results in the previous section to the analysis of a linearized flow past a rotating obstacle. In particular, we shall give simple proofs of certain $L^{q}$-estimates originally established by FARWIG, HishidA, and MÜlleR in [9] and by FARWig in [6].

Consider a rigid body in a Navier-Stokes liquid that fills the whole three-dimensional space outside the body. Assume the body's center of mass is moving with constant velocity $\lambda \mathrm{e}_{1} \in \mathbb{R}^{3}$, and that the body is rotating with constant angular velocity $\omega \mathrm{e}_{1} \in \mathbb{R}^{3}$. Here, $\lambda \in \mathbb{R}$ and $\omega \in \mathbb{R}$ are constants. We assume $\omega \neq 0$, that is, the angular velocity is non-zero. If we denote the domain exterior to the body by $\Omega$, the equations of motion for the liquid written in a frame attached to the body's center of mass are

$$
\begin{cases}\partial_{t} v-\Delta v+\nabla p+v \cdot \nabla v-\lambda \partial_{1} v-\omega\left(\mathrm{e}_{1} \wedge x \cdot \nabla v-\mathrm{e}_{1} \wedge v\right)=f & \text { in } \Omega \times(0, \infty), \\ \operatorname{div} v=0 & \text { in } \Omega \times(0, \infty), \\ v=\lambda \mathrm{e}_{1}+\left(\omega \mathrm{e}_{1}\right) \wedge x & \text { on } \partial \Omega \times(0, \infty), \\ \lim _{|x| \rightarrow \infty} v(x, t)=0, & \end{cases}
$$

where $v: \Omega \times(0, \infty) \rightarrow \mathbb{R}^{3}$ and $p: \Omega \times(0, \infty) \rightarrow \mathbb{R}$ denote the Eulerian velocity and pressure of the liquid, respectively, and $f: \Omega \times(0, \infty) \rightarrow \mathbb{R}^{3}$ is an external force. The reader is referred to [20] and [21] for the derivation of (3.87) and further details on the physical background.

In the following, we are interested in the steady-state solutions to (3.87), that is, time-independent solutions $(v, p)$ corresponding to a time-independent external force $f$. A fundamental component in the analysis of these solutions is an investigation of the corresponding linearized, steady-state, whole-space problem

$$
\begin{cases}-\Delta v+\nabla p-\lambda \partial_{1} v-\omega\left(\mathrm{e}_{1} \wedge x \cdot \nabla v-\mathrm{e}_{1} \wedge v\right)=f & \text { in } \mathbb{R}^{3}, \\ \operatorname{div} v=0 & \text { in } \mathbb{R}^{3} .\end{cases}
$$

The above system is the classical steady-state Oseen $(\lambda>0)$ or Stokes $(\lambda=0)$ problem in the whole-space with the additional term $\omega\left(\mathrm{e}_{1} \wedge x \cdot \nabla v-\mathrm{e}_{1} \wedge v\right)$ in the field equation. The additional term stems from the rotating frame of reference and represents the main challenge of the problem. Due to the unbounded coefficient $\mathrm{e}_{1} \wedge x$, the term cannot in general be treated as a perturbation to the Oseen or Stokes operator.

Maximal regularity in an $L^{q}$-setting of the system (3.88) was established for the first time in the Stokes case $(\lambda=0)$ by FArwig, Hishida, and Müller in [9], and in the Oseen case $(\lambda \neq 0)$ by FARWIG in [6]. More specifically, in [9, Theorem 1.1] the following result was obtained:

Theorem 3.7.1. Let $q \in(1, \infty), \lambda=0$, and $\omega>0$. For any $f \in L^{q}\left(\mathbb{R}^{3}\right)^{3}$ there exists a solution $(v, p) \in D^{2, q}\left(\mathbb{R}^{3}\right)^{3} \times D^{1, q}\left(\mathbb{R}^{3}\right)$ to (3.88) that satisfies

$$
\left\|\nabla^{2} v\right\|_{q}+\|\nabla p\|_{q}+\left\|\omega\left(\mathrm{e}_{1} \wedge x \cdot \nabla v-\mathrm{e}_{1} \wedge v\right)\right\|_{q} \leq C_{13}\|f\|_{q} .
$$


If $(\tilde{v}, \tilde{p}) \in D^{2, r}\left(\mathbb{R}^{3}\right)^{3} \times D^{1, r}\left(\mathbb{R}^{3}\right), r \in(1, \infty)$, is another solution to (3.88), then

$$
\tilde{v}=v+\alpha \mathrm{e}_{1}+\beta \mathrm{e}_{1} \wedge x+\sigma\left(-2 x_{1}, x_{2}, x_{3}\right), \quad \tilde{p}=p+\gamma
$$

for some $\alpha, \beta, \sigma, \gamma \in \mathbb{R}$.

In [6, Theorem 1.1 and Corollary 1.2] similar results were obtained the Oseen case:

Theorem 3.7.2. Let $q \in(1, \infty), \lambda>0$, and $\omega>0$. For any $f \in L^{q}\left(\mathbb{R}^{3}\right)^{3}$ there exists a solution $(v, p) \in D^{2, q}\left(\mathbb{R}^{3}\right)^{3} \times D^{1, q}\left(\mathbb{R}^{3}\right)$ to (3.88) that satisfies

$$
\left\|\nabla^{2} v\right\|_{q}+\|\nabla p\|_{q} \leq C_{14}\|f\|_{q} .
$$

Moreover,

$$
\left\|\lambda \partial_{1} v\right\|_{q}+\left\|\omega\left(\mathrm{e}_{1} \wedge x \cdot \nabla v-\mathrm{e}_{1} \wedge v\right)\right\|_{q} \leq C_{15}\|f\|_{q} .
$$

If $1<q<4$, then

$$
\|\nabla v\|_{\frac{4 q}{4-q}} \leq C_{16}\|f\|_{q}
$$

If $1<q<2$, then

$$
\|v\|_{\frac{2 q}{2-q}} \leq C_{17}\|f\|_{q}
$$

If $(\tilde{v}, \tilde{p}) \in D^{2, r}\left(\mathbb{R}^{3}\right)^{3} \times D^{1, r}\left(\mathbb{R}^{3}\right), 1<r<\infty$, is another solution to (3.88), then

$$
\tilde{v}=v+\alpha \mathrm{e}_{1}+\beta \mathrm{e}_{1} \wedge x, \quad \tilde{p}=p+\gamma
$$

for some $\alpha, \beta, \gamma \in \mathbb{R}$.

The proofs of Theorem 3.7.1 and Theorem 3.7.2 presented in [9] and [6], respectively, are very technical and rely on a non-trivial application of the Littlewood-Paley decomposition. Here, simple proofs based on the maximal regularity results for the time-periodic problem obtained in the previous section will be presented.

Before proceeding with the proofs, we briefly examine the connection between system (3.88) and the time-periodic problem (3.24) treated in the previous section. For this purpose, we introduce the rotation matrix

$$
Q(t):=\left(\begin{array}{ccc}
1 & 0 & 0 \\
0 & \cos \omega t & -\sin \omega t \\
0 & \sin \omega t & \cos \omega t
\end{array}\right) \in S O(3), \quad t \in \mathbb{R} .
$$

Clearly, $Q(t)$ is $\frac{2 \pi}{\omega}$-periodic. We now choose

$$
\mathcal{T}:=\frac{2 \pi}{\omega},
$$


and let $G$ denote the group $G:=\mathbb{R}^{3} \times \mathbb{R} / \mathcal{T} \mathbb{Z}$ as in (3.9). We introduce the operator

$$
\mathrm{K}: \mathscr{S}(G)^{3} \rightarrow \mathscr{S}(G)^{3}, \quad \mathrm{~K} u(x, t):=Q(t) u\left(Q(t)^{\top} x, t\right) .
$$

Clearly, $\mathrm{K}$ is a homeomorphism with inverse

$$
\mathrm{K}^{-1}: \mathscr{S}(G)^{3} \rightarrow \mathscr{S}(G)^{3}, \quad \mathrm{~K}^{-1} u(x, t)=Q(t)^{\top} u(Q(t) x, t) .
$$

Both $\mathrm{K}$ and its inverse $\mathrm{K}^{-1}$ extend by duality to homeomorphisms

$$
\mathrm{K}: \mathscr{S}^{\prime}(G)^{3} \rightarrow \mathscr{S}^{\prime}(G)^{3}, \quad \mathrm{~K}^{-1}: \mathscr{S}^{\prime}(G)^{3} \rightarrow \mathscr{S}^{\prime}(G)^{3} .
$$

The fundamental observation concerning the connection between system (3.88) and (3.24) are the identities

$$
\begin{aligned}
\partial_{t} \mathrm{~K} u & =\mathrm{K} \partial_{t} u-\mathrm{K}\left[\omega\left(\mathrm{e}_{1} \wedge x \cdot \nabla u-\mathrm{e}_{1} \wedge u\right)\right], \\
\Delta \mathrm{K} u & =\mathrm{K} \Delta u, \\
\partial_{1} \mathrm{~K} u & =\mathrm{K} \partial_{1} u,
\end{aligned}
$$

all of which one may verify by a direct computation. It follows from these identities that

$$
\mathrm{K}^{-1} \mathrm{~A}_{T P} \mathrm{~K} u=\mathrm{A}_{T P} u-\omega\left(\mathrm{e}_{1} \wedge x \cdot \nabla u-\mathrm{e}_{1} \wedge u\right),
$$

whence

$$
\mathcal{P} \mathrm{K}^{-1} \mathrm{~A}_{T P} \mathrm{~K} u=-\Delta \mathcal{P} u-\lambda \partial_{1} \mathcal{P} u-\omega\left(\mathrm{e}_{1} \wedge x \cdot \nabla \mathcal{P} u-\mathrm{e}_{1} \wedge \mathcal{P} u\right) .
$$

Restricted to time-independent vector fields, the differential operator in the field equation of (3.88) thus coincides with the conjugate by $\mathrm{K}$ of $\mathrm{A}_{T P}$, that is, coincides with the conjugate by $\mathrm{K}$ of the differential operator appearing in (3.24). Based on this relation, we can utilize the maximal regularity result for $\mathrm{A}_{T P}$ established in Theorem 3.6.5 to give simple proofs of Theorem 3.7.1 and Theorem 3.7.2. We first collect some useful identities in a lemma.

Lemma 3.7.3. The following identities are true as equations for operators on $\mathscr{S}^{\prime}(G)^{3}$ :

$$
\begin{aligned}
& \mathcal{P K} \mathcal{P}=\mathrm{K} \mathcal{P} \mathrm{K} \mathcal{P}=\mathrm{K}^{-1} \mathcal{P} \mathrm{K} \mathcal{P} \\
& \mathcal{P} \mathrm{K}^{-1} \mathcal{P}_{\perp} \mathrm{K} \mathcal{P}=(\mathrm{Id}-\mathcal{P} \mathrm{K}) \mathcal{P} \\
& \mathcal{P} \mathrm{K}^{-1} \mathrm{~A}_{T P} \mathcal{P} \mathrm{K} \mathcal{P}=\left(-\Delta-\lambda \partial_{1}\right) \mathcal{P} \mathrm{K} \mathcal{P}=\mathcal{P K} \mathcal{P}\left(-\Delta-\lambda \partial_{1}\right) \\
& (\mathcal{P K} \mathcal{P})^{2}=\mathcal{P} \mathrm{K} \mathcal{P} \\
& \Delta \mathcal{P K}=\mathcal{P} \mathrm{K} \Delta \\
& \mathcal{P} \mathrm{K}^{-1} \mathrm{~A}_{T P} \mathrm{~K}=\mathrm{K}^{-1} \mathrm{~A}_{T P} \mathrm{~K} \mathcal{P} .
\end{aligned}
$$

Moreover,

$$
\forall u \in \mathscr{S}^{\prime}(G)^{3}: \operatorname{div} u=0 \Rightarrow \operatorname{div} \mathrm{K} u=\operatorname{div} \mathcal{P} \mathrm{K} u=0 .
$$




\section{Linear theory}

Proof. All identities can be verified by a direct computation.

Proof of Theorem 3.7.1. Let $f \in L^{q}\left(\mathbb{R}^{3}\right)^{3}$. In view of (3.101), the goal is first to find a vector field $v \in D^{2, q}\left(\mathbb{R}^{3}\right)^{3}$ satisfying

$$
\mathcal{P} \mathrm{K}^{-1} \mathrm{~A}_{T P} \mathrm{~K} v=\mathcal{P}_{H} f, \quad \operatorname{div} v=0 .
$$

In view of (3.108), we clearly have $\mathcal{P} K \mathcal{P}_{H} f \in L_{\sigma}^{q}\left(\mathbb{R}^{3}\right)$. It is therefore well-known, see for example [17, Theorem IV.2.1], that there is a solution $u_{0} \in D^{2, q}\left(\mathbb{R}^{3}\right)^{3}$ to the whole-space Stokes problem

$$
-\Delta u_{0}=\mathcal{P K P}_{H} f, \quad \operatorname{div} u_{0}=0 .
$$

Since $\lambda=0, \mathcal{P} \mathcal{P}_{H} f=\mathcal{P}_{H} f$, and $\mathcal{P} u_{0}=u_{0}$, applying $\mathcal{P K} \mathcal{P}$ to both equations in (3.110) and utilizing (3.102),(3.104)-(3.105) as well as (3.108) we find that

$$
\mathcal{P} \mathrm{K}^{-1} \mathrm{~A}_{T P} \mathrm{~K} \mathcal{P} \mathrm{K} u_{0}=\mathcal{P} \mathrm{K} \mathcal{P}_{H} f, \quad \operatorname{div} \mathcal{P} K u_{0}=0 .
$$

Using (3.106) we also find that

$$
\left\|\Delta \mathcal{P} \mathrm{K} u_{0}\right\|_{q}=\left\|\mathcal{P} \mathrm{K} \Delta u_{0}\right\|_{q}=\left\|\mathcal{P} \mathrm{K} \mathcal{P}_{H} f\right\|_{q} \leq\left\|\mathcal{P}_{H} f\right\|_{q} .
$$

In the next step we observe that $\mathcal{P}_{\perp} \mathrm{K}_{\mathcal{P}_{H}} f \in L_{\sigma, \perp}^{q}(G)$ and put

$$
u_{1}:=\mathcal{P} \mathrm{K}^{-1} A_{\mathrm{TP}}^{-1} \mathcal{P}_{\perp} \mathrm{K} \mathcal{P}_{H} f,
$$

which is well-defined as an element in $W^{2, q}\left(\mathbb{R}^{3}\right)^{3}$ with div $u_{1}=0$ by Theorem 3.6.5. Utilizing (3.103) and (3.107), we can express $u_{1}$ as

$$
u_{1}=\mathrm{K}^{-1} A_{\mathrm{TP}}^{-1} \mathrm{~K}(\mathrm{Id}-\mathcal{P} \mathrm{K}) \mathcal{P}_{H} f .
$$

It follows that

$$
\mathcal{P} \mathrm{K}^{-1} \mathrm{~A}_{T P} \mathrm{~K} u_{1}=(\mathrm{Id}-\mathcal{P} \mathrm{K}) \mathcal{P}_{H} f, \quad \operatorname{div} u_{1}=0 .
$$

Recalling (3.106), we see that

$$
\begin{aligned}
\left\|\Delta u_{1}\right\|_{L^{q}\left(\mathbb{R}^{3}\right)} & =\left\|\mathcal{P} \mathrm{K}^{-1} \Delta A_{\mathrm{TP}}^{-1} \mathcal{P}_{\perp} \mathrm{K} \mathcal{P}_{H} f\right\|_{L^{q}\left(\mathbb{R}^{3}\right)} \\
& \leq\left\|\Delta A_{\mathrm{TP}}^{-1} \mathcal{P}_{\perp} \mathrm{K} \mathcal{P}_{H} f\right\|_{L^{q}(G)} \\
& \leq\left\|A_{\mathrm{TP}}^{-1} \mathcal{P}_{\perp} \mathrm{K} \mathcal{P}_{H} f\right\|_{W^{1,2, q}(G)} .
\end{aligned}
$$

From Theorem 3.6.5 we thus obtain the estimate

$$
\left\|\Delta u_{1}\right\|_{L^{q}\left(\mathbb{R}^{3}\right)} \leq c_{1}\left\|\mathcal{P}_{\perp} \mathrm{K} \mathcal{P}_{H} f\right\|_{L^{q}(G)} \leq c_{1}\left\|\mathcal{P}_{H} f\right\|_{L^{q}\left(\mathbb{R}^{3}\right)} .
$$

We now put

$$
v:=\mathcal{P K} u_{0}+u_{1} .
$$


Combining (3.111) and (3.114), we conclude that $v \in D^{2, q}\left(\mathbb{R}^{3}\right)^{3}$ is a solution to (3.109). By Lemma 2.3.5 there is $p \in D^{1, q}\left(\mathbb{R}^{3}\right)$ with $\nabla p=\left(\operatorname{Id}-\mathcal{P}_{H}\right) f$. We see from (3.101) that $(v, p)$ solves (3.88). Moreover, from (3.112) and (3.116) we obtain $\|\Delta v\|_{q} \leq c_{2}\|f\|_{q}$, which implies $\left\|\nabla^{2} v\right\|_{q} \leq c_{3}\|f\|_{q}$. Clearly $\|\nabla p\|_{q} \leq\|f\|_{q}$. Since

$$
\omega\left(\mathrm{e}_{1} \wedge x \cdot \nabla v-\mathrm{e}_{1} \wedge v\right)=f+\Delta v-\nabla p
$$

we can thus conclude (3.89).

It remains to show the uniqueness property. Assume $(\tilde{v}, \tilde{p}) \in D^{2, r}\left(\mathbb{R}^{3}\right)^{3} \times D^{1, r}\left(\mathbb{R}^{3}\right)$ is another solution to (3.88). Put $(V, P)=(v-\tilde{v}, p-\tilde{p})$. Then $(V, P)$ satisfies (3.88) with a homogeneous right-hand side. Applying div to both sides of the first equation in (3.88), we thus see that $\Delta P=0$. Since $P \in D^{1, q}\left(\mathbb{R}^{3}\right)+D^{1, r}\left(\mathbb{R}^{3}\right)$, it follows that $P=\gamma$ for some constant $\gamma \in \mathbb{R}$. Next, we consider $V$ as an element of $\mathscr{S}^{\prime}(G)^{3}$ and conclude from (3.98)-(3.100) that $\mathrm{A}_{T P} \mathrm{~K} V=0$. Applying $\mathcal{P}$ and $\mathcal{P}_{\perp}$ to this equation yields $\Delta \mathcal{P} K V=0$ and $\mathrm{A}_{T P} \mathcal{P}_{\perp} \mathrm{K} V=0$, respectively. By Lemma 3.59, the latter identity implies $\mathcal{P}_{\perp} \mathrm{K} V=0$. Consequently $\mathrm{K} V=\mathcal{P} \mathrm{K} V$, whence the former identity implies $V=\mathrm{K}^{-1}(A x+b)$ for some constants $A \in \mathbb{R}^{3 \times 3}$ and $b \in \mathbb{R}^{3}$. Combined with the fact that $\operatorname{div} V=0$, we conclude that

$$
V(x)=Q(t)^{\top}(A Q(t) x+b) \wedge \operatorname{Tr} A=0 .
$$

Choosing first $t=0$ in (3.117) immediately yields $V=A x+b$. Choosing then $x=0$ in (3.117) implies that $t \rightarrow Q(t)^{\top} b$ is independent on $t$. Consequently, $b=\alpha \mathrm{e}_{1}$ for some $\alpha \in \mathbb{R}$. Finally, inserting combinations of the values $t=0, t=\frac{\mathcal{T}}{4}, t=\frac{2 \mathcal{T}}{4}, t=\frac{3 \mathcal{T}}{4}$ and $x=\mathrm{e}_{1}, x=\mathrm{e}_{2}, x=\mathrm{e}_{3}$ one obtains $A=\beta \mathrm{e}_{1} \wedge x+\sigma\left(-2 x_{1}, x_{2}, x_{3}\right)$ for some $\beta, \sigma \in \mathbb{R}$. We thus conclude (3.90).

Proof of Theorem 3.7.2. The proof is similar to the proof of Theorem 3.7.1. More specifically, we construct the solution $v$ as a sum $v:=\mathcal{P K} u_{0}+u_{1}$ with $u_{0}$ a solution to the Oseen problem

$$
-\Delta u_{0}-\lambda \partial_{1} u_{0}=\mathcal{P} \mathrm{K} \mathcal{P}_{H} f, \quad \operatorname{div} u_{0}=0
$$

and

$$
u_{1}:=\mathcal{P} \mathrm{K}^{-1} A_{\mathrm{TP}}^{-1} \mathcal{P}_{\perp} \mathrm{K} \mathcal{P}_{H} f .
$$

Moreover, we choose $p \in D^{1, q}\left(\mathbb{R}^{3}\right)$ with $\nabla p=\left(\operatorname{Id}-\mathcal{P}_{H}\right) f$. Clearly $\|\nabla p\|_{q} \leq\|f\|_{q}$. It is well-known, see for example [17, Theorem VII.4.1], that the solution $u_{0}$ to the Oseen problem satisfies the estimate

$$
\left\|\nabla^{2} u_{0}\right\|_{q} \leq c_{1}\left\|\mathcal{P} \mathrm{KP}_{H} f\right\|_{q} \leq c_{1}\|f\|_{q} .
$$

In addition, $u_{0}$ can be chosen such that it also satisfies (3.93) and (3.94). It is easy to verify that the same estimates are also true for $\mathcal{P K} u_{0}$. As in (3.115), we utilize Theorem 3.6.5 to estimate

$$
\left\|u_{1}\right\|_{W^{2, q}\left(\mathbb{R}^{3}\right)} \leq c_{2}\|f\|_{q} .
$$




\section{Linear theory}

By Sobolev's embedding theorem it follows that $u_{1}$ also satisfies (3.93) and (3.94). We conclude that the solution $(v, p)$ satisfies (3.91), (3.93) and (3.94). It remains to show (3.92). We recall (3.98) and (3.102) to deduce

$$
0=\partial_{t} \mathcal{P} \mathrm{K} u_{0}=\partial_{t} \mathrm{~K} \mathcal{P} \mathrm{K} u_{0}=\omega\left(\mathrm{e}_{1} \wedge x \cdot \nabla \mathcal{P} \mathrm{K} u_{0}-\mathrm{e}_{1} \wedge \mathcal{P} \mathrm{K} u_{0}\right) .
$$

We also see that

$$
\partial_{t} \mathrm{~K} u_{1}=-\mathrm{K}\left[\omega\left(\mathrm{e}_{1} \wedge x \cdot \nabla u_{1}-\mathrm{e}_{1} \wedge u_{1}\right)\right] .
$$

From (3.113) we recall

$$
\mathrm{K} u_{1}=A_{\mathrm{TP}}^{-1} \mathrm{~K}(\mathrm{Id}-\mathcal{P} \mathrm{K}) \mathcal{P}_{H} f
$$

whence we can employ Theorem 3.6.5 to estimate

$$
\begin{aligned}
\left\|\omega\left(\mathrm{e}_{1} \wedge x \cdot \nabla u_{1}-\mathrm{e}_{1} \wedge u_{1}\right)\right\|_{L^{q}\left(\mathbb{R}^{3}\right)} & =\left\|\partial_{t} \mathrm{~K} u_{1}\right\|_{L^{q}(G)} \\
& \leq\left\|A_{\mathrm{TP}}^{-1} \mathrm{~K}(\mathrm{Id}-\mathcal{P} \mathrm{K}) \mathcal{P}_{H} f\right\|_{W^{1,2, q}(G)} \\
& \leq c_{3}\left\|\mathrm{~K}(\mathrm{Id}-\mathcal{P} \mathrm{K}) \mathcal{P}_{H} f\right\|_{L^{q}(G)} \\
& \leq c_{3}\|f\|_{L^{q}\left(\mathbb{R}^{3}\right)} .
\end{aligned}
$$

Combining (3.119) and (3.120) we conclude

$$
\left\|\omega\left(\mathrm{e}_{1} \wedge x \cdot \nabla v-\mathrm{e}_{1} \wedge v\right)\right\|_{L^{q}\left(\mathbb{R}^{3}\right)} \leq c_{3}\|f\|_{L^{q}\left(\mathbb{R}^{3}\right)} .
$$

Since

$$
\lambda \partial_{1} v=-\Delta v+\nabla p-\omega\left(\mathrm{e}_{1} \wedge x \cdot \nabla v-\mathrm{e}_{1} \wedge v\right)-f,
$$

we can finally conclude (3.92).

To show the uniqueness statement, we proceed as in the proof of Theorem 3.7.1 and put $(V, P)=(v-\tilde{v}, p-\tilde{p})$. As in the proof of Theorem 3.7.1, we immediately deduce $P=\gamma$ for some constant $\gamma \in \mathbb{R}$. Also as in the proof of Theorem 3.7.1, we deduce $\mathrm{A}_{T P} \mathrm{~K} V=0$, from which $\left[\Delta+\lambda \partial_{1}\right] \mathcal{P} \mathrm{K} V=0$ and $\mathrm{A}_{T P} \mathcal{P}_{\perp} \mathrm{K} V=0$ follow by applying $\mathcal{P}$ and $\mathcal{P}_{\perp}$, respectively. From $\left[\Delta+\lambda \partial_{1}\right] \mathcal{P} K V=0$ we infer that $\mathcal{P} K V=A x+b$ with $A \mathrm{e}_{1}=0$ for some constants $A \in \mathbb{R}^{3 \times 3}$ and $b \in \mathbb{R}^{3}$. By Lemma 3.6.4 it follows from $\mathrm{A}_{T P} \mathcal{P}_{\perp} \mathrm{K} V=0$ that $\mathcal{P}_{\perp} \mathrm{K} V=0$, which then implies $\mathrm{K} V=\mathcal{P} \mathrm{K} V$. Recalling that also $\operatorname{div} V=0$, we thus have

$$
V(x)=Q(t)^{\top}(A Q(t) x+b) \quad \wedge \quad A \mathrm{e}_{1}=0 \quad \wedge \quad \operatorname{Tr} A=0 .
$$

Inserting in (3.121) combinations of the values $t=0, t=\frac{\mathcal{T}}{4}, t=\frac{2 \mathcal{T}}{4}, t=\frac{3 \mathcal{T}}{4}$ and $x=0$, $x=\mathrm{e}_{1}, x=\mathrm{e}_{2}, x=\mathrm{e}_{3}$, one obtains $b=\alpha \mathrm{e}_{1}$ and $A=\beta \mathrm{e}_{1} \wedge x$ for some $\alpha, \beta \in \mathbb{R}$. We thus conclude (3.95). 
Remark 3.7.4. Recently, simple proofs of Theorem 3.7.1 and Theorem 3.7.2 based on a different approach than above have been established by GALDI and KYED in [24]. In [31] Hishida established $L^{q}$-estimates of weak solutions to (3.88) in the Stokes case $(\lambda=0)$ in terms of data $f$ in the homogeneous Sobolev space $D_{0}^{-1, q}\left(\mathbb{R}^{3}\right)^{3}$ of negative order. Similar estimates for weak solutions in the Oseen case $(\lambda \neq 0)$ were established in [37] and [38] by KraČmar, NeČAsová, and Penel. The results in [31] and [37], [38] were obtained using the same very technical approach that was used in [9] and [6] based on the Littlewood-Paley decomposition. Simple proofs of these estimates were recently made available by GALDI and KYED in [22].

Remark 3.7.5. In Theorem 3.7.1 and Theorem 3.7.2 we have stayed silent on how the various constants in the estimates depend on the parameters $\lambda$ and $\omega$. In fact, the constant $C_{13}$ in Theorem 3.7.1 and $C_{14}$ Theorem 3.7.2 are independent on $\lambda$ and $\omega$. This was already shown in [9], [6] and [24]. In the proof presented above, the independence is not clear. To establish it, one must analyze how the constant $c_{1}$ in the estimate (3.116) depends on $\lambda$ and $\omega$. This constant stems from Theorem 3.6.5 and ultimately from the application of Marcinkiewicz's multiplier theorem, or some other multiplier theorem, to the multiplier

$$
m: \mathbb{R}^{3} \times \mathbb{R} \rightarrow \mathbb{C}, \quad m(\xi, \eta):=\frac{|\xi|^{2} \cdot\left(1-\chi\left(\frac{\mathcal{T}}{2 \pi} \eta\right)\right)}{|\xi|^{2}+i\left(\eta-\lambda \xi_{1}\right)}
$$

In the Stokes case $(\lambda=0)$ it is easy to verify from Marcinkiewicz's multiplier theorem that the constant is independent on $\lambda$ and $\omega$. In the Oseen case $(\lambda \neq 0)$ this is not obvious, and a more delicate investigation of the multiplier is required. The constants $C_{15}, C_{16}$, and $C_{17}$ in Theorem 3.7.2 do depend on $\lambda$ and $\omega$. To determine the dependence more accurately, in addition to the constant $c_{1}$ in (3.116) also the constant $c_{3}$ in (3.120) and the corresponding multiplier originating from Theorem 3.6.5 must be analyzed. It seems difficult, however, to obtain with this approach the same sharp estimates that were obtain by simple means in [24]. 



\section{Strong solutions}

In this chapter, existence of strong solutions to the fully non-linear time-periodic Navier-Stokes system is established for sufficiently small data. More precisely, we shall show existence of a solution to

$$
\begin{cases}\partial_{t} u-\Delta u-\lambda \partial_{1} u+\nabla \mathfrak{p}+u \cdot \nabla u=f & \text { in } \mathbb{R}^{3} \times \mathbb{R}, \\ \operatorname{div} u=0 & \text { in } \mathbb{R}^{3} \times \mathbb{R}, \\ \lim _{|x| \rightarrow \infty} u(x, t)=0, & \\ u(\cdot, t+\mathcal{T})=u(\cdot, t) & \end{cases}
$$

for time-periodic data

$$
f(\cdot, t+\mathcal{T})=f(\cdot, t) .
$$

The solution will be strong in the sense that the solution itself and all its derivatives of the order appearing in (4.1), that is, order one in time and two in space for $u$ and one in space for $\mathfrak{p}$, are summable in certain $L^{q}$-spaces. Moreover, if $f$ is smooth also the solution is smooth.

We shall focus on the case $\lambda \neq 0$, as the case $\lambda=0$ has already been treated by other authors; see Section 1.2. We will employ the function spaces and maximal regularity results from the linear theory presented in Chapter 3 to establish existence of a solution to (4.1) by means of the contraction mapping principle.

\subsection{Existence}

As in Section 3.2, we reformulate (4.1) in a setting of $G$-defined vector fields. More specifically, we let $G$ denote the group $G:=\mathbb{R}^{3} \times \mathbb{R} / \mathcal{T} \mathbb{Z}$ and investigate for data $f \in$ $L^{q}(G)^{3}$ resolvability of the system

$$
\begin{cases}\partial_{t} u-\Delta u-\lambda \partial_{1} u+\nabla \mathfrak{p}+u \cdot \nabla u=f & \text { in } G, \\ \operatorname{div} u=0 & \text { in } G\end{cases}
$$

in the function spaces corresponding to the maximal regularity of the linearized problem established in Theorem 3.6.9. The main challenge will be to introduce the nonlinear term $u \cdot \nabla u$ into the setting of these function spaces.

In (4.3) we have omitted the condition $\lim _{|x| \rightarrow \infty} u(x, t)=0$. This condition will be incorporated into the functional analytic setting. Observe also that the periodicity 


\section{Strong solutions}

condition $u(\cdot, t+\mathcal{T})=u(\cdot, t)$ is rendered redundant in a setting of $G$-defined vector fields.

In order to estimate the nonlinear term $u \cdot \nabla u$ in the functional analytic setting dictated by the linear theory of Chapter 3, we need the following function spaces:

Definition 4.1.1. For $q \in(1,2)$ and $r \in(1, \infty)$ we put

$$
\begin{aligned}
& \mathrm{X}_{\sigma, \text { Oseen }}^{q, r}\left(\mathbb{R}^{3}\right):=\mathrm{X}_{\sigma, \text { Oseen }}^{q}\left(\mathbb{R}^{3}\right) \cap D^{2, r}\left(\mathbb{R}^{3}\right)^{3}, \\
& \|\cdot\|_{\mathrm{X}_{\sigma, \text { Oseen }}^{q, r}}\left(\mathbb{R}^{3}\right):=\|\cdot\|_{\mathrm{X}_{\sigma, \text { Oseen }}^{q}}\left(\mathbb{R}^{3}\right)+|\cdot|_{2, r} .
\end{aligned}
$$

Note that $\left(\mathrm{X}_{\sigma, \text { Oseen }}^{q, r}\left(\mathbb{R}^{3}\right),\|\cdot\|_{\mathrm{X}_{\sigma, \text { Oseen }}^{q, r}\left(\mathbb{R}^{3}\right)}\right)$ is a Banach space. Moreover, we let

$$
\begin{aligned}
& W_{\sigma, \perp}^{1,2, q, r}(G):=W_{\sigma, \perp}^{1,2, q}(G) \cap W_{\sigma, \perp}^{1,2, r}(G), \\
& \|\cdot\|_{1,2, q, r}:=\|\cdot\|_{1,2, q}+\|\cdot\|_{1,2, r} .
\end{aligned}
$$

Observe that also $\left(W_{\sigma, \perp}^{1,2, q, r}(G),\|\cdot\|_{1,2, q, r}\right)$ is a Banach space. We further put

$$
\begin{aligned}
& \mathrm{X}_{\mathrm{P}}^{q, r}(G):=\left\{\mathfrak{p} \in L_{l o c}^{1}(G) \mid\|\mathfrak{p}\|_{\mathrm{X}_{\mathrm{P}}^{q, r}(G)}<\infty\right\} \\
& \|\mathfrak{p}\|_{\mathrm{X}_{\mathrm{P}}^{q, r}(G)}:=\|\mathfrak{p}\|_{\mathrm{X}_{\mathrm{P}}^{q}(G)}+\|\nabla \mathfrak{p}\|_{r}
\end{aligned}
$$

Finally, we define

$$
\begin{aligned}
& L^{q, r}(G):=L^{q}(G) \cap L^{r}(G), \\
& \|\cdot\|_{q, r}:=\|\cdot\|_{q}+\|\cdot\|_{r},
\end{aligned}
$$

and put

$$
\begin{aligned}
L_{\sigma}^{q, r}(G) & :=L_{\sigma}^{q}(G) \cap L_{\sigma}^{r}(G), \\
\mathscr{G}^{q, r}(G) & :=\left[\mathrm{Id}-\mathcal{P}_{H}\right] L^{q, r}(G)^{3} .
\end{aligned}
$$

We shall need the following embedding properties:

Lemma 4.1.2. Let $q \in\left(1, \frac{4}{3}\right]$ and $r \in(4, \infty)$. Then every $v \in \mathrm{X}_{\sigma, \text { Oseen }}^{q, r}\left(\mathbb{R}^{3}\right)$ satisfies

$$
\begin{aligned}
& \|\nabla v\|_{\infty} \leq C_{18}\|v\|_{\mathrm{X}_{\sigma \text { Oseen }}^{q, r}\left(\mathbb{R}^{3}\right)}, \\
& \|\nabla v\|_{r} \leq C_{19}\|v\|_{\mathrm{X}_{\sigma, \text { Oseen }}^{q, r}\left(\mathbb{R}^{3}\right)}, \\
& \|v\|_{\infty} \leq C_{20}\|v\|_{\mathrm{X}_{\sigma, \text { Oseen }}^{q, r}\left(\mathbb{R}^{3}\right)} \\
& \|\nabla v\|_{2} \leq C_{21}\|v\|_{\mathrm{X}_{\sigma, \text { Oseen }}^{q, r}}\left(\mathbb{R}^{3}\right)
\end{aligned}
$$

Moreover, every $w \in W_{\sigma, \perp}^{1,2, q, r}(G)$ satisfies

$$
\|w\|_{\infty} \leq C_{22}\|w\|_{1,2, q, r} .
$$


Proof. Recall (2.3) and observe that

$$
\|\nabla v\|_{\infty} \leq C_{3}\left(|v|_{2, r}+\|\nabla v\|_{\frac{4 q}{4-q}}\right) \leq C_{3}\|v\|_{\mathrm{X}_{\sigma, \text { Oseen }}^{q, r}\left(\mathbb{R}^{3}\right)},
$$

which implies (4.9). It follows that $\nabla v \in L^{\frac{4 q}{4-q}}\left(\mathbb{R}^{3}\right) \cap L^{\infty}\left(\mathbb{R}^{3}\right)$ and consequently, since $\frac{4 q}{4-q}<r<\infty$, by interpolation that

$$
\|\nabla v\|_{r} \leq c_{1}\left(\|\nabla v\|_{\infty}+\|\nabla v\|_{\frac{4 q}{4-q}}\right) \leq c_{1}\|v\|_{\mathrm{X}_{\sigma, \text { Oseen }}^{q, r}\left(\mathbb{R}^{3}\right)} .
$$

This shows (4.10). With (4.10) at our disposal, we again employ (2.3) and find that

$$
\|v\|_{\infty} \leq C_{3}\left(\|\nabla v\|_{r}+\|v\|_{\frac{2 q}{2-q}}\right) \leq c_{2}\|v\|_{\mathrm{X}_{\sigma, \text { Oseen }}^{q, r}\left(\mathbb{R}^{3}\right)} .
$$

Thus (4.11) follows. To show (4.12), observe, since $q \leq \frac{4}{3}$ and thus $\frac{4 q}{4-q} \leq 2$, that

$$
\|\nabla v\|_{2} \leq c_{3}\left(\|\nabla v\|_{\frac{4 q}{4-q}}+\|\nabla v\|_{\infty}\right) \leq c_{4}\|v\|_{\mathrm{X}_{\sigma, \text { Oseen }}^{q, r}\left(\mathbb{R}^{3}\right)}
$$

Finally, the Sobolev embedding $W^{1, r}(G) \hookrightarrow L^{\infty}(G)$ for $r>4$ implies (4.13).

In the context of the function spaces in Definition 4.1.1, we can state the following modified version of Theorem 3.6.7:

Theorem 4.1.3. If $q \in(1,2), r \in(4, \infty)$, and $\lambda>0$, then

$$
\begin{aligned}
& A_{\lambda, \sigma}: \mathrm{X}_{\sigma, \text { Oseen }}^{q, r}\left(\mathbb{R}^{3}\right) \times W_{\sigma, \perp}^{1,2, q, r}(G) \rightarrow L_{\sigma}^{q, r}(G), \\
& A_{\lambda, \sigma}(v, w):=\partial_{t} w-\Delta(v+w)-\lambda \partial_{1}(v+w)
\end{aligned}
$$

is a homeomorphism, and

$$
\left\|A_{\lambda, \sigma}^{-1}\right\| \leq C_{23} P(\lambda, \mathcal{T})
$$

where $P(\lambda, \mathcal{T})$ is a polynomial in $\lambda$ and $\mathcal{T}$, and $C_{23}=C_{23}(q)$.

Proof. We only need to verify that $A_{\text {Oseen }}$ maps $\mathrm{X}_{\sigma, \text { Oseen }}^{q, r}\left(\mathbb{R}^{3}\right)$ homeomorphically onto $L_{\sigma}^{q, r}\left(\mathbb{R}^{3}\right)$. The rest of the proof follows exactly the proof of Theorem 3.6.7. Recalling (4.10), we have $\left\|\partial_{1} v\right\|_{r} \leq c_{1}\|v\|_{\mathrm{X}_{\sigma, \text { Oseen }}^{q, r}\left(\mathbb{R}^{3}\right)}$. It is therefore easy to verify that $A_{\text {Oseen }}$ is a continuous map from $\mathrm{X}_{\sigma, \text { Oseen }}^{q, r}\left(\mathbb{R}^{3}\right)$ into $L_{\sigma}^{q, r}\left(\mathbb{R}^{3}\right)$. It is well-known, see for example [17, Theorem VII.4.1], that the map is onto. By Lemma 3.6.2, the map is injective. Hence, $A_{\text {Oseen }}$ maps $\mathrm{X}_{\sigma, \text { Oseen }}^{q, r}\left(\mathbb{R}^{3}\right)$ homeomorphically onto $L_{\sigma}^{q, r}\left(\mathbb{R}^{3}\right)$ by the open mapping theorem.

We further state the following simple modification of Lemma 3.6.8.

Lemma 4.1.4. Let $q \in(1,3)$ and $r \in(1, \infty)$. Then

$$
\operatorname{grad}: \mathrm{X}_{\mathrm{P}}^{q, r}(G) \rightarrow \mathscr{G}^{q, r}(G), \quad \operatorname{grad} \mathfrak{p}:=\nabla \mathfrak{p}
$$

is a homeomorphism. 


\section{Strong solutions}

Proof. The proof follows the proof of Lemma 3.6.8.

Based on Theorem 4.1.3, we can now establish existence of a strong solution to (4.3) by employing the contraction mapping principle.

Theorem 4.1.5. Let $q \in\left(1, \frac{4}{3}\right], r \in(4, \infty)$, and $\lambda>0$. There is a constant $\varepsilon_{0}>0$ such that for all $f \in L^{q}(G)^{3} \cap L^{r}(G)^{3}$ with

$$
\|f\|_{q}+\|f\|_{r} \leq \varepsilon_{0}
$$

there is a solution $(u, \mathfrak{p})$ to (4.3) with $u=v+w$ and

$$
(v, w, \mathfrak{p}) \in \mathrm{X}_{\sigma, \text { Oseen }}^{q, r}\left(\mathbb{R}^{3}\right) \times W_{\sigma, \perp}^{1,2, q, r}(G) \times \mathrm{X}_{\mathrm{P}}^{q, r}(G) .
$$

Proof. We can use the Helmholtz projection, see Definition 3.2.2 and Lemma 3.2.3, to eliminate the pressure term $\nabla \mathfrak{p}$ in (4.3). More precisely, we shall first study

$$
\begin{cases}\partial_{t} u-\Delta u-\lambda \partial_{1} u+\mathcal{P}_{H}[u \cdot \nabla u]=\mathcal{P}_{H} f & \text { in } G, \\ \operatorname{div} u=0 & \text { in } G .\end{cases}
$$

After solving (4.19), a pressure term $\mathfrak{p}$ can be constructed such that $(u, \mathfrak{p})$ solves (4.3).

We first show that any pair of vector fields $(v, w) \in \mathrm{X}_{\sigma, \text { Oseen }}^{q, r}\left(\mathbb{R}^{3}\right) \times W_{\sigma, \perp}^{1,2, q, r}(G)$ satisfies $(v+w) \cdot \nabla(v+w) \in L^{q, r}(G)^{3}$. Recalling (4.11) and (4.10), we find that

$$
\|v \cdot \nabla v\|_{r} \leq\|v\|_{\infty}\|\nabla v\|_{r} \leq c_{1}\|v\|_{\mathrm{X}_{\sigma, \text { Oseen }}^{q, r}\left(\mathbb{R}^{3}\right)}^{2}
$$

Moreover, employing Hölder's inequality and recalling (4.12) we deduce

$$
\|v \cdot \nabla v\|_{q} \leq\|v\|_{\frac{2 q}{2-q}}\|\nabla v\|_{2} \leq c_{2}\|v\|_{\mathrm{X}_{\sigma, \text { Oseen }}^{q, r}\left(\mathbb{R}^{3}\right)}^{2} .
$$

We also observe that

$$
\|v \cdot \nabla w\|_{r} \leq\|v\|_{\infty}\|\nabla w\|_{r} \leq c_{3}\|v\|_{\mathrm{X}_{\sigma, \text { Oseen }}^{q, r}\left(\mathbb{R}^{3}\right)}\|w\|_{1,2, q, r}
$$

and

$$
\|v \cdot \nabla w\|_{q} \leq\|v\|_{\infty}\|\nabla w\|_{q} \leq c_{4}\|v\|_{\mathrm{X}_{\sigma, \text { Oseen }}^{q, r}\left(\mathbb{R}^{3}\right)}\|w\|_{1,2, q, r}
$$

Similarly, we can estimate

$$
\|w \cdot \nabla v\|_{r} \leq\|w\|_{r}\|\nabla v\|_{\infty} \leq c_{5}\|w\|_{1,2, q, r}\|v\|_{\mathrm{X}_{\sigma, \text { Oseen }}^{q, r}\left(\mathbb{R}^{3}\right)}
$$

and

$$
\|w \cdot \nabla v\|_{q} \leq\|w\|_{q}\|\nabla v\|_{\infty} \leq c_{6}\|w\|_{1,2, q, r}\|v\|_{\mathrm{X}_{\sigma, \text { Oseen }}^{q, r}\left(\mathbb{R}^{3}\right)}
$$


By (4.13) it follows that also

$$
\|w \cdot \nabla w\|_{r} \leq\|w\|_{\infty}\|\nabla w\|_{r} \leq c_{7}\|w\|_{1,2, q, r}^{2}
$$

and

$$
\|w \cdot \nabla v\|_{q} \leq\|w\|_{\infty}\|\nabla v\|_{q} \leq c_{8}\|w\|_{1,2, q, r}^{2} .
$$

Combining (4.20)-(4.27), we conclude

$$
\|(v+w) \cdot \nabla(v+w)\|_{q, r} \leq c_{9}\|(v, w)\|_{\mathrm{X}_{\sigma, \text { Oseen }}^{q, r}\left(\mathbb{R}^{3}\right) \times W_{\sigma, \perp}^{1,2, q, r}(G)}^{2} .
$$

We can now define the map

$$
\begin{aligned}
& \mathcal{L}: \mathrm{X}_{\sigma, \text { Oseen }}^{q, r}\left(\mathbb{R}^{3}\right) \times W_{\sigma, \perp}^{1,2, q, r}(G) \rightarrow \mathrm{X}_{\sigma, \text { Oseen }}^{q, r}\left(\mathbb{R}^{3}\right) \times W_{\sigma, \perp}^{1,2, q, r}(G), \\
& \mathcal{L}(v, w):=A_{\lambda, \sigma}^{-1}\left(\mathcal{P}_{H} f-\mathcal{P}_{H}[(v+w) \cdot \nabla(v+w)]\right) .
\end{aligned}
$$

Clearly, a fixed point $(v, w)$ of $\mathcal{L}$ induces a solution $u:=v+w$ to (4.19). We shall employ Banach's fixed point theorem to show existence of such a fixed point. To this end, we recall (4.15) and estimate

$$
\begin{aligned}
\|\mathcal{L}(v, w)\| & \leq C_{23} P(\lambda, \mathcal{T})\left(\left\|\mathcal{P}_{H} f\right\|_{q, r}+\left\|\mathcal{P}_{H}[(v+w) \cdot \nabla(v+w)]\right\|_{q, r}\right) \\
& \leq c_{10} P(\lambda, \mathcal{T})\left(\varepsilon_{0}+\|(v, w)\|_{\mathrm{X}_{\sigma, \text { Oseen }}^{q, r}}^{2}\left(\mathbb{R}^{3}\right) \times W_{\sigma, \perp}^{1,2, q, r}(G)\right.
\end{aligned} .
$$

Consequently, $\mathcal{L}$ is a self-mapping on the ball $\overline{B_{\rho}} \subset \mathrm{X}_{\sigma, \text { Oseen }}^{q, r}\left(\mathbb{R}^{3}\right) \times W_{\sigma, \perp}^{1,2, q, r}(G)$ provided $\rho$ and $\varepsilon_{0}$ satisfy

$$
c_{10} P(\lambda, \mathcal{T})\left(\varepsilon_{0}+\rho^{2}\right) \leq \rho .
$$

The above inequality is satisfied if we, for example, choose

$$
\rho:=\frac{1}{4 c_{10} P(\lambda, \mathcal{T})}, \quad \varepsilon_{0}:=\frac{1}{16 c_{10}^{2} P(\lambda, \mathcal{T})^{2}} .
$$

With this choice of parameters, we further have for $\left(v_{1}, w_{1}\right),\left(v_{2}, w_{2}\right) \in \overline{B_{\rho}}$ :

$$
\begin{aligned}
\left\|\mathcal{L}\left(v_{1}, w_{1}\right)-\mathcal{L}\left(v_{2}, w_{2}\right)\right\| & \leq c_{10} P(\lambda, \mathcal{T})\left\|\left(v_{1}, w_{1}\right)-\left(v_{2}, w_{2}\right)\right\|^{2} \\
& \leq c_{10} P(\lambda, \mathcal{T}) 2 \rho\left\|\left(v_{1}, w_{1}\right)-\left(v_{2}, w_{2}\right)\right\| \\
& \leq \frac{1}{2}\left\|\left(v_{1}, w_{1}\right)-\left(v_{2}, w_{2}\right)\right\| .
\end{aligned}
$$

Thus, $\mathcal{L}$ becomes a contractive self-mapping. By Banach's fixed point theorem, $\mathcal{L}$ then has a unique fixed point in $\overline{B_{\rho}}$.

Finally, we construct the pressure. By (4.28), $u \cdot \nabla u \in L^{q, r}(G)^{3}$. Recalling Lemma 4.1.4, the function

$$
\mathfrak{p}:=\operatorname{grad}^{-1}\left(\left[\operatorname{Id}-\mathcal{P}_{H}\right](f-u \cdot \nabla u)\right)
$$

belongs to $\mathrm{X}_{\mathrm{P}}^{q, r}(G)$. Clearly, $(u, \mathfrak{p})$ is a solution to (4.3). 


\subsection{Regularity}

We shall now show for a strong solution that additional regularity of the data translates into a similar degree of additional regularity for the solution. More specifically, we have

Theorem 4.2.1. Let $\lambda>0, q \in\left(1, \frac{4}{3}\right], r \in(8, \infty), m \in \mathbb{N}_{0}$, and

$$
f \in W^{m, q}(G)^{3} \cap W^{m, r}(G)^{3} .
$$

If $(u, \mathfrak{p})$ is a solution to (4.3) with $u=v+w$ and

$$
(v, w, \mathfrak{p}) \in \mathrm{X}_{\sigma, \text { Oseen }}^{q, r}\left(\mathbb{R}^{3}\right) \times W_{\sigma, \perp}^{1,2, q, r}(G) \times \mathrm{X}_{\mathrm{P}}^{q, r}(G),
$$

then

$$
\begin{aligned}
& \forall(\alpha, \beta, \kappa) \in \mathbb{N}_{0}^{3} \times \mathbb{N}_{0}^{3} \times \mathbb{N}_{0},|\alpha| \leq m,|\beta|+|\kappa| \leq m: \\
&\left(\partial_{x}^{\alpha} v, \partial_{x}^{\beta} \partial_{t}^{\kappa} w, \partial_{x}^{\beta} \partial_{t}^{\kappa} \mathfrak{p}\right) \in \mathrm{X}_{\sigma, \text { Oseen }}^{q, r}\left(\mathbb{R}^{3}\right) \times W_{\sigma, \perp}^{1,2, q, r}(G) \times \mathrm{X}_{\mathrm{P}}^{q, r}(G) .
\end{aligned}
$$

Proof. Observe that

$$
\begin{cases}\partial_{t} w-\Delta w-\lambda \partial_{1} w=\mathcal{P}_{H} \mathcal{P}_{\perp} f-\mathcal{P}_{H}\left[\mathcal{P}_{\perp}[w \cdot \nabla w]+w \cdot \nabla v+v \cdot \nabla w\right] & \text { in } G \\ \operatorname{div} w=0 & \text { in } G .\end{cases}
$$

We shall first "take half a derivative in time" on both sides of (4.34). We therefore introduce the pseudo-differential operator

$$
\partial_{t}^{\frac{1}{2}}: \mathscr{S}(G) \rightarrow \mathscr{S}(G), \quad \partial_{t}^{\frac{1}{2}} \psi:=\mathcal{F}_{G}^{-1}\left[\left(i \frac{2 \pi}{\mathcal{T}} k\right)^{\frac{1}{2}} \widehat{\psi}\right]
$$

which, by duality, extends to an operator

$$
\partial_{t}^{\frac{1}{2}}: \mathscr{S}^{\prime}(G) \rightarrow \mathscr{S}^{\prime}(G)
$$

Note that $w \cdot \nabla w=\operatorname{div} w \otimes w$. We thus find that

$$
\begin{aligned}
\partial_{t}^{\frac{1}{2}}\left[\mathcal{P}_{\perp}[w \cdot \nabla w]\right]_{j} & =\mathcal{F}_{G}^{-1}\left[\frac{\left(1-\kappa_{0}(\xi, k)\right)\left(i \frac{2 \pi}{\mathcal{T}} k\right)^{\frac{1}{2}}\left(i \xi_{l}\right)}{|\xi|^{2}+i \frac{2 \pi}{\mathcal{T}} k}\left(|\xi|^{2}+i \frac{2 \pi}{\mathcal{T}} k\right) \widehat{w_{j} w_{l}}\right] \\
& =\mathcal{F}_{G}^{-1}\left[M_{l}(\xi, k) \mathcal{F}_{G}\left[\left(\partial_{t}-\Delta\right)\left[w_{j} w_{l}\right]\right]\right]
\end{aligned}
$$

with

$$
M_{l}: \widehat{G} \rightarrow \mathbb{C}, \quad M_{l}(\xi, k):=\frac{\left(1-\kappa_{0}(\xi, k)\right)\left(i \frac{2 \pi}{\mathcal{T}} k\right)^{\frac{1}{2}}\left(i \xi_{l}\right)}{|\xi|^{2}+i \frac{2 \pi}{\mathcal{T}} k} .
$$

Observe that the only zero of the polynomial denumerator of $M_{l}$ is $(\xi, k)=(0,0)$. When $k=0$, however, the numerator vanishes due to the term $\left(1-\kappa_{0}(\xi, k)\right)$. Consequently, we 
see that $M_{l} \in C^{\infty}(\widehat{G})$ and that $M_{l}$ is bounded. Using the same argument based on the transference principle of multipliers that was employed in the proof of Theorem 3.6.7, it follows that $M_{l}$ is an $L^{p}(G)$-multiplier for all $p \in(1, \infty)$. More specifically, let $\chi$ be a "cut-off" function with

$$
\chi \in C_{0}^{\infty}(\mathbb{R} ; \mathbb{R}), \quad \chi(\eta)=1 \text { for }|\eta| \leq \frac{1}{2}, \quad \chi(\eta)=0 \text { for }|\eta| \geq 1,
$$

and define

$$
m_{l}: \mathbb{R}^{3} \times \mathbb{R} \rightarrow \mathbb{C}, \quad m(\xi, \eta):=\frac{\left(1-\chi\left(\frac{\mathcal{T}}{2 \pi} \eta\right)\right)(i \eta)^{\frac{1}{2}} \xi_{l}}{|\xi|^{2}+i \eta}
$$

Since $m_{l}$ vanishes in a neighborhood of $\{\eta=0\}$, we see that $m_{l}$ is smooth. Moreover, as one readily verifies, $m_{l}$ satisfies condition (2.7) in Marcinkiewicz's multiplier theorem. Consequently, $m_{l}$ is an $L^{p}\left(\mathbb{R}^{3} \times \mathbb{R}\right)$-multiplier. Introducing the continuous homomorphism $\Phi: \widehat{G} \rightarrow \mathbb{R}^{3} \times \mathbb{R}$ as in (3.67), and observing that $M_{l}=m_{l} \circ \Phi$, we obtain from Theorem 3.4.1 that $M_{l}$ is an $L^{p}(G)$-multiplier. It follows from (4.35) that

$$
\forall p \in(1, \infty): \quad\left\|\partial_{t}^{\frac{1}{2}}\left[\mathcal{P}_{\perp}[w \cdot \nabla w]\right]\right\|_{p} \leq c_{1}\left\|\left(\partial_{t}-\Delta\right)[w \otimes w]\right\|_{p}
$$

Due to $w \in W_{\sigma, \perp}^{1,2, q, r}(G)$ and the fact that, by $(4.13), w \in L^{\infty}(G)$, we have

$$
\partial_{t} w_{j} w_{l} \in L^{q}(G) \cap L^{r}(G), \quad \Delta w_{j} w_{l} \in L^{q}(G) \cap L^{r}(G) .
$$

Moreover, since $\frac{r}{2}>q$ we observe that

$$
\nabla w_{j} \cdot \nabla w_{l} \in L^{q}(G) \cap L^{\frac{r}{2}}(G) .
$$

Computing

$$
\left(\partial_{t}-\Delta\right)\left[w_{j} w_{l}\right]=\partial_{t} w_{j} w_{l}+w_{j} \partial_{t} w_{l}-\left(\Delta w_{j} w_{l}+w_{j} \Delta w_{l}+2 \nabla w_{j} \cdot \nabla w_{l}\right),
$$

we conclude by (4.36) that

$$
\partial_{t}^{\frac{1}{2}}\left[\mathcal{P}_{\perp}[w \cdot \nabla w]\right] \in L^{q}(G) \cap L^{\frac{r}{2}}(G) .
$$

We now recall (4.9), (4.11), and (4.13) to deduce

$$
\begin{aligned}
& \partial_{t} w_{j} v_{l} \in L^{q}(G) \cap L^{r}(G), \\
& \Delta w_{j} v_{l} \in L^{q}(G) \cap L^{r}(G), \\
& \Delta v_{j} w_{l} \in L^{q}(G) \cap L^{r}(G), \\
& \nabla w_{j} \cdot \nabla v_{l} \in L^{q}(G) \cap L^{r}(G) .
\end{aligned}
$$

By the same argument as above, we obtain

$$
\left\|\partial_{t}^{\frac{1}{2}}[w \cdot \nabla v+v \cdot \nabla w]\right\|_{q} \in L^{q}(G) \cap L^{r}(G) \subset L^{q}(G) \cap L^{\frac{r}{2}}(G) .
$$




\section{Strong solutions}

We now apply $\partial_{t}^{\frac{1}{2}}$ to both sides in (4.34). Clearly, all differential operators commute with $\partial_{t}^{\frac{1}{2}}$. Recalling Definition 3.2.2 of the Helmholtz projection in terms of a Fourier multiplier, we also see that $\partial_{t}^{\frac{1}{2}}$ commutes with $\mathcal{P}_{H}$. Similarly, $\partial_{t}^{\frac{1}{2}}$ commutes with $\mathcal{P}_{\perp}$. Consequently, after applying $\partial_{t}^{\frac{1}{2}}$ to both sides in (4.34), we obtain

$$
\partial_{t}\left[\partial_{t}^{\frac{1}{2}} w\right]-\Delta\left[\partial_{t}^{\frac{1}{2}} w\right]-\lambda \partial_{1}\left[\partial_{t}^{\frac{1}{2}} w\right] \in L_{\sigma, \perp}^{q}(G) \cap L_{\sigma, \perp}^{\frac{r}{2}}(G) .
$$

Combining now Theorem 3.6.5 and Lemma 3.6.4, we conclude

$$
\partial_{t}^{\frac{1}{2}} w \in W_{\sigma, \perp}^{1,2, q}(G) \cap W_{\sigma, \perp}^{1,2, \frac{r}{2}}(G) .
$$

Since

$$
\partial_{t} \partial_{j} w=\mathcal{F}_{G}^{-1}\left[\frac{\left(1-\kappa_{0}(\xi, k)\right)\left(i \frac{2 \pi}{\mathcal{T}} k\right)^{\frac{1}{2}}\left(i \xi_{j}\right)}{|\xi|^{2}+i \frac{2 \pi}{\mathcal{T}} k} \mathcal{F}_{G}\left[\left(\partial_{t}-\Delta\right) \partial_{t}^{\frac{1}{2}} w_{j}\right]\right],
$$

we deduce, by analyzing the multiplier

$$
(\xi, k) \rightarrow \frac{\left(1-\kappa_{0}(\xi, k)\right)\left(i \frac{2 \pi}{\mathcal{T}} k\right)^{\frac{1}{2}}\left(i \xi_{j}\right)}{|\xi|^{2}+i \frac{2 \pi}{\mathcal{T}} k}
$$

in same way as we analyzed $M_{l}$, that

$$
\forall p \in(1, \infty): \quad\left\|\partial_{t} \partial_{j} w\right\|_{p} \leq c_{2}\left\|\left(\partial_{t}-\Delta\right) \partial_{t}^{\frac{1}{2}} w_{j}\right\|_{p}
$$

In view of (4.39), we thus have

$$
\partial_{t} \partial_{j} w \in L^{q}(G) \cap L^{\frac{r}{2}}(G) .
$$

Combined with the fact that $w \in W^{1,2, q, r}(G)$, it follows that $\nabla w \in W^{1, \frac{r}{2}}(G)$. Since $\frac{r}{2}>4$, classical Sobolev embedding yields $W^{1, \frac{r}{2}}(G) \hookrightarrow L^{\infty}(G)$. Thus

$$
\nabla w \in L^{\infty}(G) .
$$

With this information, we return to (4.37) and conclude that in fact

$$
\nabla w_{h} \cdot \nabla w_{m} \in L^{q}(G) \cap L^{r}(G) .
$$

We therefore obtain improved regularity in (4.38), namely

$$
\partial_{t}^{\frac{1}{2}}\left[\mathcal{P}_{\perp}[w \cdot \nabla w]\right] \in L^{q}(G) \cap L^{r}(G) .
$$

Repeating the argument leading up to (4.40), we then deduce

$$
\partial_{t} \partial_{j} w \in L^{q}(G) \cap L^{r}(G) .
$$


We shall now take a full derivative in time on both sides in (4.34). Concerning the terms that will then appear on the right-hand side, we observe, recalling (4.41), (4.13), (4.9) and (4.11), and (4.44) that

$$
\begin{aligned}
& \partial_{t} w \cdot \nabla w \in L^{q}(G) \cap L^{r}(G), \\
& w \cdot \nabla \partial_{t} w \in L^{q}(G) \cap L^{r}(G), \\
& \partial_{t} w \cdot \nabla v \in L^{q}(G) \cap L^{r}(G), \\
& v \cdot \nabla \partial_{t} w \in L^{q}(G) \cap L^{r}(G) .
\end{aligned}
$$

Consequently, we have

$$
\partial_{t}\left[\partial_{t} w\right]-\Delta\left[\partial_{t} w\right]-\lambda \partial_{1}\left[\partial_{t} w\right] \in L_{\sigma, \perp}^{q}(G) \cap L_{\sigma, \perp}^{r}(G) .
$$

Combining again Theorem 3.6.5 and Lemma 3.6.4, we conclude the improved regularity

$$
\partial_{t} w \in W_{\sigma, \perp}^{1,2, q}(G) \cap W_{\sigma, \perp}^{1,2, r}(G)
$$

of the time derivative of $w$. We can establish the same improved regularity of spatial derivatives of $w$. For this purpose we simply observe that

$$
\begin{aligned}
& \partial_{j} w \cdot \nabla w \in L^{q}(G) \cap L^{r}(G), \\
& w \cdot \nabla \partial_{j} w \in L^{q}(G) \cap L^{r}(G), \\
& \partial_{j} w \cdot \nabla v \in L^{q}(G) \cap L^{r}(G), \\
& w \cdot \nabla \partial_{j} v \in L^{q}(G) \cap L^{r}(G), \\
& \partial_{j} v \cdot \nabla w \in L^{q}(G) \cap L^{r}(G), \\
& v \cdot \nabla \partial_{j} w \in L^{q}(G) \cap L^{r}(G),
\end{aligned}
$$

which implies, by applying $\partial_{j}$ on both sides in (4.41), that

$$
\partial_{t}\left[\partial_{j} w\right]-\Delta\left[\partial_{j} w\right]-\lambda \partial_{1}\left[\partial_{j} w\right] \in L_{\sigma, \perp}^{q}(G) \cap L_{\sigma, \perp}^{r}(G) .
$$

Employing yet again Theorem 3.6.5 and Lemma 3.6.4, we obtain

$$
\nabla w \in W_{\sigma, \perp}^{1,2, q}(G) \cap W_{\sigma, \perp}^{1,2, r}(G) .
$$

We now turn our attention to $v$. Note that

$$
\begin{cases}-\Delta v-\lambda \partial_{1} v=\mathcal{P}_{H} \mathcal{P} f-\mathcal{P}_{H}[\mathcal{P}[w \cdot \nabla w]+v \cdot \nabla v] & \text { in } \mathbb{R}^{3} \\ \operatorname{div} v=0 & \text { in } \mathbb{R}^{3}\end{cases}
$$

Recalling (4.41), (4.13), (4.9) and (4.11), one readily verifies

$$
\begin{aligned}
& \mathcal{P}\left[\partial_{j} w \cdot \nabla w\right] \in L^{q}\left(\mathbb{R}^{3}\right) \cap L^{r}\left(\mathbb{R}^{3}\right), \\
& \mathcal{P}\left[w \cdot \nabla \partial_{j} w\right] \in L^{q}\left(\mathbb{R}^{3}\right) \cap L^{r}\left(\mathbb{R}^{3}\right), \\
& \partial_{j} v \cdot \nabla v \in L^{q}\left(\mathbb{R}^{3}\right) \cap L^{r}\left(\mathbb{R}^{3}\right), \\
& v \cdot \nabla \partial_{j} v \in L^{q}\left(\mathbb{R}^{3}\right) \cap L^{r}\left(\mathbb{R}^{3}\right) .
\end{aligned}
$$




\section{Strong solutions}

Thus, applying $\partial_{j}$ on both sides in (4.47) we obtain

$$
-\Delta\left[\partial_{j} v\right]-\lambda \partial_{1}\left[\partial_{j} v\right] \in L_{\sigma}^{q}\left(\mathbb{R}^{3}\right) \cap L_{\sigma}^{r}\left(\mathbb{R}^{3}\right) .
$$

By Theorem 3.6.1 and Lemma 3.6.2, we conclude that

$$
\nabla v \in \mathrm{X}_{\sigma, \text { Oseen }}^{q, r}\left(\mathbb{R}^{3}\right) .
$$

Summarizing (4.45), (4.46), and (4.48), we have established similar regularity for the first order derivatives of $w$ and $v$ as we had originally for $w$ and $v$. More precisely, we have

$$
\forall|\alpha| \leq 1,|\beta|+|\kappa| \leq 1: \quad\left(\partial_{x}^{\alpha} v, \partial_{x}^{\beta} \partial_{t}^{\kappa} w\right) \in \mathrm{X}_{\sigma, \text { Oseen }}^{q, r}\left(\mathbb{R}^{3}\right) \times W_{\sigma, \perp}^{1,2, q, r}(G)
$$

Iterating the argument above with $\left(\partial_{x}^{\alpha} v, \partial_{x}^{\beta} \partial_{t}^{\kappa} w\right)$ in the role of $(v, w)$, we obtain the same regularity for all higher order derivatives as well, that is,

$$
\forall|\alpha| \leq m,|\beta|+|\kappa| \leq m: \quad\left(\partial_{x}^{\alpha} v, \partial_{x}^{\beta} \partial_{t}^{\kappa} w\right) \in \mathrm{X}_{\sigma, \text { Oseen }}^{q, r}\left(\mathbb{R}^{3}\right) \times W_{\sigma, \perp}^{1,2, q, r}(G) .
$$

Concerning the pressure term $\mathfrak{p}$, we clearly have

$$
\nabla \mathfrak{p}=\left(\mathrm{Id}-\mathcal{P}_{H}\right)[f-u \cdot \nabla u] .
$$

From (4.49) one easily deduces

$$
\forall|\beta|+|\kappa| \leq m: \quad \partial_{x}^{\beta} \partial_{t}^{\kappa}[u \cdot \nabla u] \in L^{q, r}(G) .
$$

Taking derivatives in (4.50) and recalling Lemma 4.1.4, we thus obtain

$$
\forall|\beta|+|\kappa| \leq m: \quad \partial_{x}^{\beta} \partial_{t}^{\kappa} \mathfrak{p} \in \mathrm{X}_{\mathrm{P}}^{q, r}(G),
$$

which concludes the theorem.

Provided the data $f$ is smooth, one can use Theorem 4.2.1 to show that a strong solution to (4.3) is also smooth.

Corollary 4.2.2. Let $\lambda>0, q \in\left(1, \frac{4}{3}\right], r \in(8, \infty)$, and

$$
f \in L^{r, q}(G)^{3} \cap C^{\infty}(G) .
$$

If $(u, \mathfrak{p})$ is a solution to (4.3) with $u=v+w$ and

$$
(v, w, \mathfrak{p}) \in \mathrm{X}_{\sigma, \text { Oseen }}^{q, r}\left(\mathbb{R}^{3}\right) \times W_{\sigma, \perp}^{1,2, q, r}(G) \times \mathrm{X}_{\mathrm{P}}^{q, r}(G),
$$

then $u \in C^{\infty}(G)^{3}$ and $\mathfrak{p} \in C^{\infty}(G)$.

Proof. The corollary follows from Theorem 4.2 .1 by a standard localization argument combined with classical Sobolev embedding. 
Improved regularity in the sense of integrability can be established for a solution to (4.3) in the same way we showed additional differentiability in Theorem 4.2.1. We state one such result in the following lemma.

Lemma 4.2.3. Let $\lambda>0, q_{0} \in\left(1, \frac{4}{3}\right), r \in(4, \infty)$, and

$$
\forall q \in\left(1, q_{0}\right]: \quad f \in L^{q, r}(G)^{3} .
$$

If $(u, \mathfrak{p})$ is a solution to (4.3) with $u=v+w$ and

$$
(v, w, \mathfrak{p}) \in \mathrm{X}_{\sigma, \text { Oseen }}^{q_{0}, r}\left(\mathbb{R}^{3}\right) \times W_{\sigma, \perp}^{1,2, q_{0}, r}(G) \times \mathrm{X}_{\mathrm{P}}^{q_{0}, r}(G),
$$

then

$$
\forall q \in\left(1, q_{0}\right]: \quad(v, w, \mathfrak{p}) \in \mathrm{X}_{\sigma, \text { Oseen }}^{q, r}\left(\mathbb{R}^{3}\right) \times W_{\sigma, \perp}^{1,2, q, r}(G) \times \mathrm{X}_{\mathrm{P}}^{q, r}(G) .
$$

Proof. As in the proof of Theorem 4.2.1, we consider $w$ and $v$ separately. More precisely, we utilize that $w$ solves

$$
\begin{cases}\partial_{t} w-\Delta w-\lambda \partial_{1} w=\mathcal{P}_{H} \mathcal{P}_{\perp} f-\mathcal{P}_{H}\left[\mathcal{P}_{\perp}[w \cdot \nabla w]+w \cdot \nabla v+v \cdot \nabla w\right] & \text { in } G \\ \operatorname{div} w=0 & \text { in } G\end{cases}
$$

and that $v$ solves

$$
\begin{cases}-\Delta v-\lambda \partial_{1} v=\mathcal{P}_{H} \mathcal{P} f-\mathcal{P}_{H}[\mathcal{P}[w \cdot \nabla w]+v \cdot \nabla v] & \text { in } \mathbb{R}^{3} \\ \operatorname{div} v=0 & \text { in } \mathbb{R}^{3}\end{cases}
$$

Recalling Lemma 4.1.2, one can easily verify that

$$
w \cdot \nabla w \in L^{1}(G) \cap L^{q_{0}}(G), \quad w \cdot \nabla v \in L^{1}(G) \cap L^{q_{0}}(G), \quad v \cdot \nabla w \in L^{1}(G) \cap L^{q_{0}}(G) .
$$

Thus, from Theorem 3.6.5 and Lemma 3.6.4 it follows that

$$
\forall q \in\left(1, q_{0}\right]: \quad w \in W_{\sigma, \perp}^{1,2, q, r}(G) .
$$

Based on (4.57), we shall show improved regularity of $v$ in the same way. The critical term in this context is $v \cdot \nabla v$. Observe for $v \in \mathrm{X}_{\sigma, \text { Oseen }}^{q, r}\left(\mathbb{R}^{3}\right)$ that

$$
s=\frac{4 q}{8-3 q} \quad \Longrightarrow \quad v \cdot \nabla v \in L^{s}(G)
$$

Employing Theorem 3.6.1 and Lemma 3.6.2 in a bootstrapping argument along the sequence

$$
q_{n+1}:=\frac{4 q_{n}}{8-3 q_{n}}, n \in \mathbb{N}_{0}
$$




\section{Strong solutions}

yields the desired property of $v$. Note that since $q_{0} \in\left(1, \frac{4}{3}\right)$ the sequence is strictly decreasing towards zero. It follows that

$$
\forall q \in\left(1, q_{0}\right]: \quad v \in \mathrm{X}_{\sigma, \text { Oseen }}^{q, r}\left(\mathbb{R}^{3}\right) .
$$

The statement concerning the pressure follows from Lemma 4.1.4 and the fact that

$$
\nabla \mathfrak{p}=\left(\mathrm{Id}-\mathcal{P}_{H}\right)[f-w \cdot \nabla w-w \cdot \nabla v-v \cdot \nabla w-v \cdot \nabla v]
$$

\subsection{Energy equation}

The fluid flow corresponding to the strong solution from Theorem 4.1.5 satisfies a certain balance of energy. The energy being dissipated in the fluid due to viscosity over a period of time equals the energy added to the fluid from the external force. This property is expressed in the energy equation in the following theorem.

Theorem 4.3.1. Let $q \in\left(1, \frac{4}{3}\right], r \in(4, \infty), \lambda>0$, and $f \in L^{q}(G)^{3} \cap L^{r}(G)^{3}$. A solution $(u, \mathfrak{p})$ to (4.3) with $u=v+w$ and

$$
(v, w, \mathfrak{p}) \in \mathrm{X}_{\sigma, \text { Oseen }}^{q, r}\left(\mathbb{R}^{3}\right) \times W_{\sigma, \perp}^{1,2, q, r}(G) \times \mathrm{X}_{\mathrm{P}}^{q, r}(G)
$$

satisfies

$$
\frac{1}{\mathcal{T}} \int_{0}^{\mathcal{T}} \int_{\mathbb{R}^{3}}|\nabla u|^{2} \mathrm{~d} x \mathrm{~d} t=\frac{1}{\mathcal{T}} \int_{0}^{\mathcal{T}} \int_{\mathbb{R}^{3}} f \cdot u \mathrm{~d} x \mathrm{~d} t .
$$

Proof. The proof relies on the summability properties of the solution being sufficient to multiply (4.3) with $u$ itself and subsequently integrate over space and time. Due to the dissimilar summability properties of $v$ and $w$, it is more convenient to carry out this process for $v$ and $w$ separately. More specifically, we first multiply

$$
\partial_{t} w-\Delta w-\lambda \partial_{1} w=\mathcal{P}_{H} \mathcal{P}_{\perp} f-\mathcal{P}_{H}\left[\mathcal{P}_{\perp}[w \cdot \nabla w]+w \cdot \nabla v+v \cdot \nabla w\right]
$$

with $w$ and integrate over $G$. We can easily verify that the product of $w$ with each term in (4.60) is integrable over $G$. For example, we observe that

$$
\frac{1}{\mathcal{T}} \int_{0}^{\mathcal{T}} \int_{\mathbb{R}^{3}}\left|\partial_{t} w \cdot w\right| \mathrm{d} x \mathrm{~d} t \leq\left\|\partial_{t} w\right\|_{4}\|w\|_{\frac{4}{3}} \leq\|w\|_{1,2, q, r}^{2} .
$$


Similarly, one can verify for all the other terms in (4.60) that the product with $w$ can be integrated over $G$. We thus conclude that

$$
\begin{aligned}
& \frac{1}{\mathcal{T}} \int_{0}^{\mathcal{T}} \int_{\mathbb{R}^{3}} \partial_{t} w \cdot w-\Delta w \cdot w-\lambda \partial_{1} w \cdot w \mathrm{~d} x \mathrm{~d} t \\
& \quad=\frac{1}{\mathcal{T}} \int_{0} \int_{\mathbb{R}^{3}} \mathcal{P}_{\perp} f \cdot w-\mathcal{P}_{\perp}[w \cdot \nabla w] \cdot w-(w \cdot \nabla v) \cdot w-(v \cdot \nabla w) \cdot w \mathrm{~d} x \mathrm{~d} t,
\end{aligned}
$$

where the Helmholtz projection $\mathcal{P}_{H}$ can be omitted since $w$ is solenoidal. Since $w=\mathcal{P}_{\perp} w$, we can, recalling (3.48), also omit the projection $\mathcal{P}_{\perp}$ in the first two terms on the righthand side. Moreover, the summability properties of $w$ are sufficient to integrate by parts in each term above. Consequently, we obtain

$$
\frac{1}{\mathcal{T}} \int_{0}^{\mathcal{T}} \int_{\mathbb{R}^{3}} \nabla w: \nabla w \mathrm{~d} x \mathrm{~d} t=\frac{1}{\mathcal{T}} \int_{0}^{\mathcal{T}} \int_{\mathbb{R}^{3}} f \cdot w-(w \cdot \nabla v) \cdot w \mathrm{~d} x \mathrm{~d} t .
$$

We now repeat the procedure with $v$ in the role of $w$, that is, we multiply

$$
-\Delta v-\lambda \partial_{1} v=\mathcal{P}_{H} \mathcal{P} f-\mathcal{P}_{H}[\mathcal{P}[w \cdot \nabla w]+v \cdot \nabla v]
$$

with $v$ and integrate over $\mathbb{R}^{3}$. Again it should be verified that the product of the terms in (4.63) with $v$ is integrable over $\mathbb{R}^{3}$. This, however, is standard to show. For example, in view of (4.11) and the fact that $\frac{2 q}{2-q} \leq \frac{q}{q-1}$ it follows that

$$
\left|\int_{\mathbb{R}^{3}} \Delta v \cdot v \mathrm{~d} x\right| \leq\|\Delta v\|_{q}\|v\|_{\frac{q}{q-1}} \leq\|v\|_{\mathrm{X}_{\sigma, \text { Oseen }}^{q, r}}^{2}\left(\mathbb{R}^{3}\right) .
$$

Similarly, one can verify for all the other terms in (4.63) that the product with $v$ can be integrated over $\mathbb{R}^{3}$. We thus conclude that

$$
\int_{\mathbb{R}^{3}}-\Delta v \cdot v-\lambda \partial_{1} v \cdot v \mathrm{~d} x=\int_{\mathbb{R}^{3}} f \cdot v-(w \cdot \nabla w) \cdot v-(v \cdot \nabla v) \cdot v \mathrm{~d} x .
$$

One may also verify that the summability properties of $v$ are sufficient to integrate by parts in (4.64). We thereby obtain

$$
\int_{\mathbb{R}^{3}} \nabla v: \nabla v \mathrm{~d} x=\int_{\mathbb{R}^{3}} f \cdot v+(w \cdot \nabla v) \cdot w \mathrm{~d} x .
$$

Adding together (4.62) and (4.65) we deduce

$$
\frac{1}{\mathcal{T}} \int_{0}^{\mathcal{T}} \int_{\mathbb{R}^{3}}|\nabla w|^{2}+|\nabla v|^{2} \mathrm{~d} x \mathrm{~d} t=\frac{1}{\mathcal{T}} \int_{0}^{\mathcal{T}} \int_{\mathbb{R}^{3}} f \cdot(v+w) \mathrm{d} x \mathrm{~d} t .
$$


Since

$$
\frac{1}{\mathcal{T}} \int_{0}^{\mathcal{T}} \int_{\mathbb{R}^{3}} \nabla v: \nabla w \mathrm{~d} x \mathrm{~d} t=0
$$

we finally conclude (4.59).

\subsection{Uniqueness}

We shall now show that the strong solution to (4.3) from Theorem 4.1.5 is unique in a very large class of vector fields. The class coincides with a natural function space for weak solutions. In fact, in Chapter 6 the existence of at least one weak solution in this class will be shown without imposing any restrictions on the data.

In order to characterize the class in which uniqueness can be established, we introduce to notion of a physically reasonable weak solution.

Definition 4.4.1. Let $q \in\left(1, \frac{5}{4}\right], r \in(4, \infty)$, and $\lambda>0$. For $f \in L^{q}(G)^{3} \cap L^{r}(G)^{3}$ we say that $\mathcal{U} \in L_{l o c}^{1}(G)^{3}$ is a physically reasonable weak solution to (4.3) if

1. $\mathcal{U} \in L^{2}\left(\mathbb{T} ; D_{0, \sigma}^{1,2}\left(\mathbb{R}^{3}\right)\right)$,

2. $\mathcal{P}_{\perp} \mathcal{U} \in L^{\infty}\left(\mathbb{T} ; L^{2}\left(\mathbb{R}^{3}\right)^{3}\right)$,

3. $\mathcal{U}$ is a generalized solution to (4.3), that is, for all $\Phi \in C_{0, \sigma}^{\infty}(G)$

$$
\frac{1}{\mathcal{T}} \int_{0}^{\mathcal{T}} \int_{\mathbb{R}^{3}}-\mathcal{U} \cdot \partial_{t} \Phi+\nabla \mathcal{U}: \nabla \Phi-\lambda \partial_{1} \mathcal{U} \cdot \Phi+(\mathcal{U} \cdot \nabla \mathcal{U}) \cdot \Phi \mathrm{d} x \mathrm{~d} t=\frac{1}{\mathcal{T}} \int_{0}^{\mathcal{T}} \int_{\mathbb{R}^{3}} f \cdot \Phi \mathrm{d} x \mathrm{~d} t .
$$

4. $\mathcal{U}$ satisfies the energy inequality

$$
\frac{1}{\mathcal{T}} \int_{0}^{\mathcal{T}} \int_{\mathbb{R}^{3}}|\nabla \mathcal{U}|^{2} \mathrm{~d} x \mathrm{~d} t \leq \frac{1}{\mathcal{T}} \int_{0}^{\mathcal{T}} \int_{\mathbb{R}^{3}} f \cdot \mathcal{U} \mathrm{d} x \mathrm{~d} t
$$

Remark 4.4.2. The integral on the right-hand side of (4.68) is well-defined as consequence of Lemma 4.4.4 below.

Remark 4.4.3. The characterization of a solution satisfying 1-4 as a physically reasonable weak solution is justified by the properties concerning the energy of the corresponding fluid flow that can be derived from $\mathcal{P}_{\perp} \mathcal{U} \in L^{\infty}\left(\mathbb{T} ; L^{2}\left(\mathbb{R}^{3}\right)^{3}\right)$ and (4.68). More precisely, the former property implies that the kinetic energy of the time-dependent part of the flow is bounded, while the latter states that the energy dissipated due to the viscosity of the fluid is less than the input of energy from the external forces. It is not clear at the outset if a generalized solution satisfies these physically reasonable properties. However, in Chapter 6 the existence of at least one generalized solution that satisfies these properties will be shown. 
Employing the linear theory from Chapter 3, we can establish additional regularity for weak solutions.

Lemma 4.4.4. Let $\mathcal{U} \in L^{2}\left(\mathbb{T} ; D_{0, \sigma}^{1,2}\left(\mathbb{R}^{3}\right)\right)$ with $\mathcal{P}_{\perp} \mathcal{U} \in L^{\infty}\left(\mathbb{T} ; L^{2}\left(\mathbb{R}^{3}\right)^{3}\right)$ be a generalized solution to (4.3) with $\lambda>0$, that is, it satisfies (4.67). If for some $q \in\left(1, \frac{5}{4}\right]$

$$
f \in L^{q}(G)^{3} \cap L^{\frac{3}{2}}(G)^{3}
$$

then

$$
\mathcal{P}_{\perp} \mathcal{U} \in W_{\sigma, \perp}^{1,2, q}(G)
$$

If for some $\tilde{q} \in\left(1, \frac{3}{2}\right]$

$$
f \in L^{\tilde{q}}(G)^{3} \cap L^{\frac{3}{2}}(G)^{3}
$$

then

$$
\mathcal{P U} \in \mathrm{X}_{\sigma, \text { Oseen }}^{\tilde{q}}\left(\mathbb{R}^{3}\right) .
$$

Proof. We first assume (4.69) for some $q \in\left(1, \frac{5}{4}\right]$. Put $\mathcal{V}:=\mathcal{P U}$ and $\mathcal{W}:=\mathcal{P}_{\perp} \mathcal{U}$. By assumption $\mathcal{W} \in L^{2}(G)^{3}$ and $\nabla \mathcal{W} \in L^{2}(G)^{3}$, whence

$$
\mathcal{W} \cdot \nabla \mathcal{W} \in L^{1}(G)^{3} .
$$

Hölder's inequality together with Lemma 2.3.1 invoked with $q=2$ and $r=\frac{10}{3}$ yields

$$
\begin{aligned}
\frac{1}{\mathcal{T}} \int_{0}^{\mathcal{T}} \int_{\mathbb{R}^{3}}|\mathcal{W} \cdot \nabla \mathcal{W}|^{\frac{5}{4}} \mathrm{~d} x \mathrm{~d} t & \leq \frac{1}{\mathcal{T}} \int_{0}^{\mathcal{T}}\|\nabla \mathcal{W}(\cdot, t)\|_{2}^{\frac{5}{4}}\|\mathcal{W}(\cdot, t)\|_{\frac{10}{3}}^{\frac{5}{4}} \mathrm{~d} t \\
& \leq c_{1} \frac{1}{\mathcal{T}} \int_{0}^{\mathcal{T}}\|\nabla \mathcal{W}(\cdot, t)\|_{2}^{\frac{5}{4}}\left(\|\nabla \mathcal{W}(\cdot, t)\|_{2}^{\frac{3}{5}}\|\mathcal{W}(\cdot, t)\|_{2}^{\frac{2}{5}}\right)^{\frac{5}{4}} \mathrm{~d} t \\
& \leq c_{2}\left(\underset{t \in \mathbb{T}}{\operatorname{ess} \sup }\|\mathcal{W}(\cdot, t)\|_{2}\right)^{\frac{1}{2}} \cdot \frac{1}{\mathcal{T}} \int_{0}^{\mathcal{T}}\|\nabla \mathcal{W}(\cdot, t)\|_{2}^{2} \mathrm{~d} x<\infty
\end{aligned}
$$

whence

$$
\mathcal{W} \cdot \nabla \mathcal{W} \in L^{\frac{5}{4}}(G)^{3} .
$$




\section{Strong solutions}

We further deduce, by employing first Minkowski's integral inequality then Hölders inequality and finally (2.2), that

$$
\begin{aligned}
\left(\int_{\mathbb{R}^{3}}\left|\frac{1}{\mathcal{T}} \int_{0}^{\mathcal{T}} \mathcal{W} \cdot \nabla \mathcal{W} \mathrm{d} t\right|^{\frac{3}{2}} \mathrm{~d} x\right)^{\frac{3}{2}} & \leq \frac{1}{\mathcal{T}} \int_{0}^{\mathcal{T}}\left(\int_{\mathbb{R}^{3}}|\mathcal{W} \cdot \nabla \mathcal{W}|^{\frac{3}{2}} \mathrm{~d} x\right)^{\frac{3}{2}} \mathrm{~d} t \\
& \leq \frac{1}{\mathcal{T}} \int_{0}^{\mathcal{T}}\left(\int_{\mathbb{R}^{3}}|\mathcal{W}|^{6} \mathrm{~d} x\right)^{\frac{1}{6}}\left(\int_{\mathbb{R}^{3}}|\nabla \mathcal{W}|^{2} \mathrm{~d} x\right)^{\frac{1}{2}} \mathrm{~d} t \\
& \leq \frac{1}{\mathcal{T}} \int_{0}^{\mathcal{T}}|\nabla \mathcal{W}|^{2} \mathrm{~d} x \mathrm{~d} t<\infty
\end{aligned}
$$

Consequently, we have

$$
\mathcal{P}[\mathcal{W} \cdot \nabla \mathcal{W}] \in L^{\frac{3}{2}}(G)^{3} .
$$

Recalling Lemma 3.5.5, it is easy to verify from the weak formulation (4.67) that

$$
\int_{\mathbb{R}^{3}}-\mathcal{V} \cdot \partial_{t} \Phi+\nabla \mathcal{V}: \nabla \Phi-\lambda \partial_{1} \mathcal{V} \cdot \Phi+(\mathcal{V} \cdot \nabla \mathcal{V}+\mathcal{P}[\mathcal{W} \cdot \nabla \mathcal{W}]) \cdot \Phi \mathrm{d} x=\int_{\mathbb{R}^{3}} \mathcal{P} f \cdot \Phi \mathrm{d} x
$$

for all $\Phi \in C_{0, \sigma}^{\infty}\left(\mathbb{R}^{3}\right)$. This means that $\mathcal{V} \in D_{0, \sigma}^{1,2}\left(\mathbb{R}^{3}\right)$ is a generalized solution to the steady-state problem

$$
\begin{cases}-\Delta \mathcal{V}-\lambda \partial_{1} \mathcal{V}+\mathcal{P}_{H}[\mathcal{V} \cdot \nabla \mathcal{V}]=\mathcal{P}_{H} \mathcal{P} f-\mathcal{P}_{H} \mathcal{P}[\mathcal{W} \cdot \nabla \mathcal{W}] & \text { in } \mathbb{R}^{3} \\ \operatorname{div} \mathcal{V}=0 & \text { in } \mathbb{R}^{3}\end{cases}
$$

From (4.73), (4.76), and assumption (4.69), we deduce the summability property

$$
\mathcal{P} f-\mathcal{P}[\mathcal{W} \cdot \nabla \mathcal{W}] \in L^{q}\left(\mathbb{R}^{3}\right)^{3} \cap L^{\frac{3}{2}}\left(\mathbb{R}^{3}\right)^{3}
$$

for the right-hand side in (4.78). Know results for the steady-state Navier-Stokes problem (4.78) then imply

$$
\mathcal{V} \in \mathrm{X}_{\sigma, \text { Oseen }}^{q}\left(\mathbb{R}^{3}\right) \cap \mathrm{X}_{\sigma, \text { Oseen }}^{\frac{3}{2}}\left(\mathbb{R}^{3}\right) .
$$

More specifically, we can employ $[21 \text {, Lemma X.6.1 }]^{1}$ which, although formulated for a three-dimensional exterior domain, also holds for solutions to the whole space problem (4.78). By the additional regularity for $\mathcal{V}$ implied by (4.79), it follows that $\nabla \mathcal{V} \in L^{2}\left(\mathbb{R}^{3}\right)^{3}$.

\footnotetext{
${ }^{1}$ Lemma X.6.1 is new in the latest edition of the monograph [21].
} 
Since by assumption $\mathcal{W} \in L^{2}(G)^{3}$, we thus have $\mathcal{W} \cdot \nabla \mathcal{V} \in L^{1}(G)^{3}$. In addition, we can deduce as in (4.74) that $\mathcal{W} \cdot \nabla \mathcal{V} \in L^{\frac{5}{4}}(G)^{3}$. Consequently, by interpolation

$$
\mathcal{W} \cdot \nabla \mathcal{V} \in L^{q}(G)^{3} .
$$

From (4.79) we further obtain $\mathcal{V} \in L^{\frac{2 q}{2-q}}\left(\mathbb{R}^{3}\right)^{3}$, which combined with $\nabla \mathcal{W} \in L^{2}(G)^{3 \times 3}$ yields

$$
\mathcal{V} \cdot \nabla \mathcal{W} \in L^{q}(G)^{3} .
$$

We have now derived enough summability properties for the terms appearing in (4.3) to finalize the proof. Recalling again Lemma 3.5.5, it is easy to verify from the weak formulation (4.67) that

$$
\begin{aligned}
& \frac{1}{\mathcal{T}} \int_{0}^{\mathcal{T}} \int_{\mathbb{R}^{3}}-\mathcal{W} \cdot \partial_{t} \Phi+\nabla \mathcal{W}: \nabla \Phi-\lambda \partial_{1} \mathcal{W} \cdot \Phi \\
& \quad+\left(\mathcal{P}_{\perp}[\mathcal{W} \cdot \nabla \mathcal{W}]+\mathcal{W} \cdot \nabla \mathcal{V}+\mathcal{V} \cdot \nabla \mathcal{W}\right) \cdot \Phi \mathrm{d} x \mathrm{~d} t=\frac{1}{\mathcal{T}} \int_{0} \int_{\mathbb{R}^{3}} \mathcal{P}_{\perp} f \cdot \Phi \mathrm{d} x \mathrm{~d} t
\end{aligned}
$$

for all $\Phi \in C_{0, \sigma}^{\infty}(G)$. The summability of $\mathcal{W}$ and $\nabla \mathcal{W}$ together the summability properties obtained for $\mathcal{W} \cdot \nabla \mathcal{W}, \mathcal{W} \cdot \nabla \mathcal{V}$, and $\mathcal{V} \cdot \nabla \mathcal{W}$ above enables us to extend (4.82) to all $\Phi \in \mathscr{S}(G)$. Thus the system

$$
\begin{cases}\partial_{t} \mathcal{W}-\Delta \mathcal{W}-\lambda \partial_{1} \mathcal{W}=\mathcal{P}_{H} \mathcal{P}_{\perp} f-\mathcal{P}_{H}\left[\mathcal{P}_{\perp}[\mathcal{W} \cdot \nabla \mathcal{W}]+\mathcal{W} \cdot \nabla \mathcal{V}+\mathcal{V} \cdot \nabla \mathcal{W}\right] & \text { in } G \\ \operatorname{div} \mathcal{W}=0 & \text { in } G\end{cases}
$$

is satisfied as an identity in $\mathscr{S}^{\prime}(G)$. From (4.73), (4.75), (4.80), (4.81), and the assumptions on $f$, we conclude that

$$
\mathcal{P}_{H} \mathcal{P}_{\perp} f-\mathcal{P}_{H}\left[\mathcal{P}_{\perp}[\mathcal{W} \cdot \nabla \mathcal{W}]+\mathcal{W} \cdot \nabla \mathcal{V}+\mathcal{V} \cdot \nabla \mathcal{W}\right] \in L^{q}(G)^{3}
$$

Consequently, Theorem 3.6.5 combined with Lemma 3.6.4 implies (4.70).

Finally, assume (4.71) for some $\tilde{q} \in\left(1, \frac{3}{2}\right]$. In view of (4.73) and (4.76), we deduce

$$
\mathcal{P} f-\mathcal{P}[\mathcal{W} \cdot \nabla \mathcal{W}] \in L^{\tilde{q}}\left(\mathbb{R}^{3}\right)^{3} \cap L^{\frac{3}{2}}\left(\mathbb{R}^{3}\right)^{3} .
$$

Recalling that $\mathcal{V}$ solves (4.78), utilizing once more [21, Lemma X.6.1] we conclude (4.72).

We are now in a position to show that the strong solution to (4.3) from Theorem 4.1.5 is unique in the class of physically reasonable weak solutions. More specifically, with a minor restriction on the class of the data the following extension of Theorem 4.1.5 that includes the aforementioned uniqueness of the solution can be established. 


\section{Strong solutions}

Theorem 4.4.5. Let $q \in\left(1, \frac{6}{5}\right], r \in(4, \infty), \lambda>0$, and $f \in L^{q}(G)^{3} \cap L^{r}(G)^{3}$. There is a constant $\varepsilon_{1}>0$ such that if

$$
\|f\|_{q}+\|f\|_{r} \leq \varepsilon_{1}
$$

there is a solution $(u, \mathfrak{p})$ to (4.3) with $u=v+w$ and

$$
(v, w, \mathfrak{p}) \in \mathrm{X}_{\sigma, \text { Oseen }}^{q, r}\left(\mathbb{R}^{3}\right) \times W_{\sigma, \perp}^{1,2, q, r}(G) \times \mathrm{X}_{\mathrm{P}}^{q, r}(G) .
$$

Moreover, $u$ is unique in the class of physically reasonable weak solutions characterized by Definition 4.4.1.

Proof. Choosing $\varepsilon_{1} \leq \varepsilon_{0}$ we obtain a solution $(u, \mathfrak{p}), u=v+w$ in the class (4.84) from Theorem 4.1.5. From the proof of Theorem 4.1.5, in particular (4.30), we recall that $u \in \mathrm{B}_{\rho} \subset \mathrm{X}_{\sigma, \text { Oseen }}^{q, r}\left(\mathbb{R}^{3}\right) \times W_{\sigma, \perp}^{1,2, q, r}(G)$ with $\rho:=\varepsilon_{1}^{\frac{1}{2}}$, which means that

$$
\|(v, w)\|_{\mathrm{X}_{\sigma, \text { Oseen }}^{q, r}\left(\mathbb{R}^{3}\right) \times W_{\sigma, \perp}^{1,2, q, r}(G)} \leq \varepsilon^{\frac{1}{2}} .
$$

Now consider a physically reasonable weak solution $\mathcal{U}$ corresponding to the same data $f$, that is, a weak solution to (4.3) that satisfies $1-4$ of Definition 4.4.1. Put $\mathcal{V}:=\mathcal{P U}$ and $\mathcal{W}:=\mathcal{P}_{\perp} \mathcal{U}$. We shall verify that the regularity of $\mathcal{V}$ and $\mathcal{W}$ ensured by Lemma 4.4.4 enables us to use $u=v+w$ as a test function in the weak formulation for $\mathcal{U}=\mathcal{V}+\mathcal{W}$. Observe for example that (4.72) implies $\mathcal{V} \in L^{\frac{2 q}{2-q}}\left(\mathbb{R}^{3}\right)^{3}$, from which it follows, since the Hölder conjugate $\left(\frac{2 q}{2-q}\right)^{\prime}=\frac{2 q}{3 q-2}$ belongs to the interval $(q, r)$, that

$$
\mathcal{V} \cdot \partial_{t} w \in L^{1}(G)
$$

Moreover, since by assumption $\mathcal{W} \in L^{2}(G)^{3}$, we also have

$$
\mathcal{W} \cdot \partial_{t} w \in L^{1}(G) .
$$

In a similar manner, one may verify that

$$
\nabla \mathcal{V}: \nabla v, \nabla \mathcal{V}: \nabla w, \nabla \mathcal{W}: \nabla v, \nabla \mathcal{W}: \nabla w \in L^{1}(G)^{3} .
$$

From (4.72) and the initial regularity of $\mathcal{V}$, we obtain $\partial_{1} \mathcal{V} \in L^{q}\left(\mathbb{R}^{3}\right)^{3} \cap L^{2}\left(\mathbb{R}^{3}\right)^{3}$. Thus, since $v \in L^{\frac{2 q}{2-q}}\left(\mathbb{R}^{3}\right)^{3}$ and the Hölder conjugate $\left(\frac{2 q}{2-q}\right)^{\prime}=\frac{2 q}{3 q-2}$ belongs to the interval $(q, 2)$, we deduce

$$
\partial_{1} \mathcal{V} \cdot v \in L^{1}\left(\mathbb{R}^{3}\right)^{3}
$$

In view of (4.70), the same argument yields

$$
\partial_{1} \mathcal{W} \cdot v \in L^{1}\left(\mathbb{R}^{3}\right)^{3} .
$$

It is easy to see that

$$
\partial_{1} \mathcal{V} \cdot w, \partial_{1} \mathcal{W} \cdot w \in L^{1}(G)^{3}
$$


By Lemma 4.1.2, we have $v \in L^{\frac{2 q}{2-q}}\left(\mathbb{R}^{3}\right)^{3} \cap L^{\infty}\left(\mathbb{R}^{3}\right)^{3}$. Moreover, recalling (2.2), we find that $\mathcal{V} \in L^{\frac{2 q}{2-q}}\left(\mathbb{R}^{3}\right)^{3} \cap L^{6}\left(\mathbb{R}^{3}\right)^{3}$. We thus see that $v, w, \mathcal{V} \in L^{4}(G)^{3}$, from which one can deduce that

$$
(\mathcal{V} \cdot \nabla \mathcal{V}) \cdot v,(\mathcal{V} \cdot \nabla \mathcal{V}) \cdot w,(\mathcal{V} \cdot \nabla \mathcal{W}) \cdot v,(\mathcal{V} \cdot \nabla \mathcal{W}) \cdot w \in L^{1}(G)^{3}
$$

Lemma 4.1.2 also yields $w \in L^{\infty}(G)^{3}$, whence

$$
(\mathcal{W} \cdot \nabla \mathcal{V}) \cdot v,(\mathcal{W} \cdot \nabla \mathcal{V}) \cdot w,(\mathcal{W} \cdot \nabla \mathcal{W}) \cdot v,(\mathcal{W} \cdot \nabla \mathcal{W}) \cdot w \in L^{1}(G)^{3} .
$$

Finally, recalling that $\left(\frac{2 q}{2-q}\right)^{\prime}=\frac{2 q}{3 q-2} \in(q, 2)$, the summability of $f$ implies

$$
f \cdot v, f \cdot w \in L^{1}(G)^{3} .
$$

From the summability properties (4.86)-(4.94), we conclude, by a standard approximation argument, that $u=v+w$ can be used as a test function in the weak formulation for $\mathcal{U}=\mathcal{V}+\mathcal{W}$ and thus obtain

$$
\frac{1}{\mathcal{T}} \int_{0}^{\mathcal{T}} \int_{\mathbb{R}^{3}}-\mathcal{W} \cdot \partial_{t} w+\nabla \mathcal{U}: \nabla u-\lambda \partial_{1} \mathcal{U} \cdot u+(\mathcal{U} \cdot \nabla \mathcal{U}) \cdot u \mathrm{~d} x \mathrm{~d} t=\frac{1}{\mathcal{T}} \int_{0}^{\mathcal{T}} \int_{\mathbb{R}^{3}} f \cdot u \mathrm{~d} x \mathrm{~d} t
$$

We now consider the equation

$$
\partial_{t} u-\Delta u-\lambda \partial_{1} u+\nabla \mathfrak{p}+u \cdot \nabla u=f \quad \text { in } G
$$

satisfied by the strong solution. We shall multiply (4.96) with $\mathcal{U}$ and integrate over $G$. With the aid of Lemma 4.4.4 and Lemma 4.1.2, one can verify as above that the resulting integral is well-defined. We thus obtain

$$
\frac{1}{\mathcal{T}} \int_{0}^{\mathcal{T}} \int_{\mathbb{R}^{3}} \partial_{t} w \cdot \mathcal{W}-\Delta u \cdot \mathcal{U}-\lambda \partial_{1} u \cdot \mathcal{U}+\nabla \mathfrak{p} \cdot \mathcal{U}+(u \cdot \nabla u) \cdot \mathcal{U} \mathrm{d} x \mathrm{~d} t=\frac{1}{\mathcal{T}} \int_{0}^{\mathcal{T}} \int_{\mathbb{R}^{3}} f \cdot \mathcal{U} \mathrm{d} x \mathrm{~d} t
$$

Recalling (4.88)-(4.93), we see that the following integration by parts is valid

$$
\frac{1}{\mathcal{T}} \int_{0}^{\mathcal{T}} \int_{\mathbb{R}^{3}} \partial_{t} w \cdot \mathcal{W}+\nabla u: \nabla \mathcal{U}+\lambda u \cdot \partial_{1} \mathcal{U}-(u \cdot \nabla \mathcal{U}) \cdot u \mathrm{~d} x \mathrm{~d} t=\frac{1}{\mathcal{T}} \int_{0}^{\mathcal{T}} \int_{\mathbb{R}^{3}} f \cdot \mathcal{U} \mathrm{d} x \mathrm{~d} t .
$$

Adding together (4.95) and (4.97), we deduce

$$
\begin{aligned}
2 \frac{1}{\mathcal{T}} \int_{0}^{\mathcal{T}} \int_{\mathbb{R}^{3}} \nabla U: \nabla u \mathrm{~d} x \mathrm{~d} t= & \frac{1}{\mathcal{T}} \int_{0}^{\mathcal{T}} \int_{\mathbb{R}^{3}} f \cdot \mathcal{U} \mathrm{d} x \mathrm{~d} t+\frac{1}{\mathcal{T}} \int_{0}^{\mathcal{T}} \int_{\mathbb{R}^{3}} f \cdot u \mathrm{~d} x \mathrm{~d} t \\
& +\frac{1}{\mathcal{T}} \int_{0} \int_{\mathbb{R}^{3}}((u-\mathcal{U}) \cdot \nabla \mathcal{U}) \cdot u \mathrm{~d} x \mathrm{~d} t .
\end{aligned}
$$




\section{Strong solutions}

Since

$$
\frac{1}{\mathcal{T}} \int_{0}^{\mathcal{T}} \int_{\mathbb{R}^{3}}|\nabla \mathcal{U}-\nabla u|^{2} \mathrm{~d} x \mathrm{~d} t=\frac{1}{\mathcal{T}} \int_{0}^{\mathcal{T}} \int_{\mathbb{R}^{3}}|\nabla \mathcal{U}|^{2}+|\nabla u|^{2} \mathrm{~d} x \mathrm{~d} t-2 \frac{1}{\mathcal{T}} \int_{0}^{\mathcal{T}} \int_{\mathbb{R}^{3}} \nabla U: \nabla u \mathrm{~d} x \mathrm{~d} t
$$

we can utilize (4.98) in combination with the energy equality (4.59) satisfied by $u$ and the energy inequality (4.68) satisfied by $\mathcal{U}$ to deduce

$$
\frac{1}{\mathcal{T}} \int_{0}^{\mathcal{T}} \int_{\mathbb{R}^{3}}|\nabla \mathcal{U}-\nabla u|^{2} \mathrm{~d} x \mathrm{~d} t \leq \frac{1}{\mathcal{T}} \int_{0}^{\mathcal{T}} \int_{\mathbb{R}^{3}}((\mathcal{U}-u) \cdot \nabla \mathcal{U}) \cdot u \mathrm{~d} x \mathrm{~d} t .
$$

Recalling (4.12), we see that $\nabla u \in L^{2}(G)^{3}$. We already observed that $u, \mathcal{V} \in L^{4}(G)^{3}$. Thus, an integration by parts yields

$$
\frac{1}{\mathcal{T}} \int_{0}^{\mathcal{T}} \int_{\mathbb{R}^{3}}(\mathcal{V} \cdot \nabla u) \cdot u \mathrm{~d} x \mathrm{~d} t=0 .
$$

Since $\mathcal{W} \in L^{2}(G)^{3}$ and $u \in L^{\infty}(G)$, it further follows that

$$
\frac{1}{\mathcal{T}} \int_{0}^{\mathcal{T}} \int_{\mathbb{R}^{3}}(\mathcal{W} \cdot \nabla u) \cdot u \mathrm{~d} x \mathrm{~d} t=0
$$

Adding together (4.100) and (4.101) we obtain

$$
\frac{1}{\mathcal{T}} \int_{0}^{\mathcal{T}} \int_{\mathbb{R}^{3}}(\mathcal{U} \cdot \nabla u) \cdot u \mathrm{~d} x \mathrm{~d} t=0
$$

Consequently, we can rewrite (4.99) as

$$
\frac{1}{\mathcal{T}} \int_{0}^{\mathcal{T}} \int_{\mathbb{R}^{3}}|\nabla \mathcal{U}-\nabla u|^{2} \mathrm{~d} x \mathrm{~d} t \leq \frac{1}{\mathcal{T}} \int_{0}^{\mathcal{T}} \int_{\mathbb{R}^{3}}((\mathcal{U}-u) \cdot \nabla(\mathcal{U}-u)) \cdot u \mathrm{~d} x \mathrm{~d} t .
$$

Recalling (2.2), we estimate

$$
\begin{aligned}
& \left|\frac{1}{\mathcal{T}} \int_{0}^{\mathcal{T}} \int_{\mathbb{R}^{3}}((\mathcal{U}-u) \cdot \nabla(\mathcal{U}-u)) \cdot u \mathrm{~d} x \mathrm{~d} t\right| \\
& \quad \leq \frac{1}{\mathcal{T}} \int_{0}^{\mathcal{T}}\|\mathcal{U}(\cdot, t)-u(\cdot, t)\|_{6}\|\nabla \mathcal{U}(\cdot, t)-\nabla u(\cdot, t)\|_{2}\|u(\cdot, t)\|_{3} \mathrm{~d} t \\
& \quad \leq \underset{t \in \mathbb{T}}{\operatorname{ess} \sup }\|u(\cdot, t)\|_{3} \frac{1}{\mathcal{T}} \int_{0}^{\mathcal{T}}\|\nabla \mathcal{U}(\cdot, t)-\nabla u(\cdot, t)\|_{2}^{2} \mathrm{~d} t .
\end{aligned}
$$


Now we finally need the assumption $q \leq \frac{6}{5}$, which implies that $\frac{2 q}{2-q} \leq 3$. Consequently, the fact that $\|v\|_{\frac{2 q}{2-q}} \leq\|v\|_{\mathrm{X}_{\sigma, \text { Oseen }}^{q, r}\left(\mathbb{R}^{3}\right)}$ and $\|v\|_{\infty} \leq C_{20}\|v\|_{\mathrm{X}_{\sigma, \text { Oseen }}^{q, r}}\left(\mathbb{R}^{3}\right)$, the latter inequality due to Lemma 4.1.2, implies

$$
\|v\|_{\left.L^{3}\left(\mathbb{R}^{3}\right)\right)} \leq c_{1}\|v\|_{\mathrm{X}_{\sigma, \text { Oseen }}^{q, r}\left(\mathbb{R}^{3}\right)} .
$$

Since $w \in W_{\sigma, \perp}^{1,2, r, q}(G) \hookrightarrow W^{1,3}(G)^{3} \hookrightarrow W^{1,3}\left(\mathbb{T} ; L^{3}\left(\mathbb{R}^{3}\right)^{3}\right)$, standard Sobolev embedding yields $w \in L^{\infty}\left(\mathbb{T} ; L^{3}\left(\mathbb{R}^{3}\right)^{3}\right)$ with

$$
\|w\|_{L^{\infty}\left(\mathbb{T} ; L^{3}\left(\mathbb{R}^{3}\right)\right)} \leq c_{2}\|w\|_{W_{\sigma, \perp}^{1,2, q, r}(G)} .
$$

Combining (4.105) and (4.106), we obtain

$$
\|u\|_{L^{\infty}\left(\mathbb{T} ; L^{3}\left(\mathbb{R}^{3}\right)\right)} \leq c_{3}\|(v, w)\|_{\mathrm{X}_{\sigma, \text { Oseen }}^{q, r}\left(\mathbb{R}^{3}\right) \times W_{\sigma, \perp}^{1,2, q, r}(G)} .
$$

This estimate together with (4.103), (4.104), and (4.85) finally yields

$$
\frac{1}{\mathcal{T}} \int_{0}^{\mathcal{T}} \int_{\mathbb{R}^{3}}|\nabla \mathcal{U}-\nabla u|^{2} \mathrm{~d} x \mathrm{~d} t \leq c_{3} \varepsilon_{1}^{\frac{1}{2}} \frac{1}{\mathcal{T}} \int_{0}^{\mathcal{T}} \int_{\mathbb{R}^{3}}|\nabla \mathcal{U}-\nabla u|^{2} \mathrm{~d} x \mathrm{~d} t .
$$

We conclude that $\mathcal{U}=u$ if $\varepsilon_{1}<c_{3}{ }^{-\frac{1}{2}}$.

Remark 4.4.6. The proof of Theorem 4.4.5 follows an idea introduced by GALDI in [16]. The same method was also used in [53] to show a uniqueness result for the time-periodic Navier-Stokes problem in the case $\lambda=0$. 



\section{Asymptotic structure}

In this chapter, an asymptotic expansion as $|x| \rightarrow \infty$ is carried out for a solution to the time-periodic Navier-Stokes problem

$$
\begin{cases}\partial_{t} u-\Delta u-\lambda \partial_{1} u+\nabla \mathfrak{p}+u \cdot \nabla u=f & \text { in } \mathbb{R}^{3} \times \mathbb{R} \\ \operatorname{div} u=0 & \text { in } \mathbb{R}^{3} \times \mathbb{R} \\ \lim _{|x| \rightarrow \infty} u(x, t)=0, & \\ u(\cdot, t+\mathcal{T})=u(\cdot, t) & \end{cases}
$$

for time-periodic data

$$
f(\cdot, t+\mathcal{T})=f(\cdot, t)
$$

More precisely, for a strong solution as the one found in Chapter 4 an asymptotic expansion

$$
u(x, t)=\Gamma(x) \cdot \alpha+\mathscr{R}(x, t)
$$

is established. Here, $\Gamma$ is an explicitly known tensor field, $\alpha \in \mathbb{R}^{3}$ a constant that depends on the data $f$, and $\mathscr{R}(x, t)$ a remainder term that decays faster than $\Gamma$ as $|x| \rightarrow \infty$. We shall focus on the case $\lambda \neq 0$ as the case $\lambda=0$ has already been dealt with by other authors; see Section 1.2.

In Section 5.1 we establish an asymptotic expansion of a solution to (5.1). We shall then, in Section 5.2, discuss an important property concerning the kinetic energy of the fluid flow corresponding to $u$. As we shall see, properties of this energy can be derived from the asymptotic expansion. Finally, in Section 5.3, we apply the results from Section 5.1 to derive an asymptotic expansion of flow past a rotating body.

\subsection{Asymptotic expansion}

Once more, we reformulate the system (4.1) in a setting of $G$-defined vector fields, that is, we consider the group $G:=\mathbb{R}^{3} \times \mathbb{R} / \mathcal{T} \mathbb{Z}$ and study the system

$$
\begin{cases}\partial_{t} u-\Delta u-\lambda \partial_{1} u+\nabla \mathfrak{p}+u \cdot \nabla u=f & \text { in } G, \\ \operatorname{div} u=0 & \text { in } G .\end{cases}
$$

We start by showing the following mild, yet very significant, decay property of $\nabla u$ in the case $f$ has compact support. The lemma below, including the proof, is a modified version of [18, Lemma IX.8.2]. 
Lemma 5.1.1. Let $f \in C_{0}(G)^{3}$ and $(u, \mathfrak{p})$ be a solution to (5.4) with

$$
\begin{aligned}
& u \in C^{2}(G)^{3}, \quad \mathfrak{p} \in C^{1}(G), \quad \nabla u \in L^{2}(G)^{3 \times 3} \cap L^{\frac{3}{2}}(G)^{3 \times 3}, \\
& \forall s \in(2,3]: u \in L^{s}(G)^{3}, \quad \exists s^{\prime} \in[3 / 2,2): \mathfrak{p} \in L^{s^{\prime}}(G) .
\end{aligned}
$$

Then

$$
\forall \varepsilon>0: \quad \frac{1}{\mathcal{T}} \int_{0}^{\mathcal{T}} \int_{\mathrm{B}^{R}}|\nabla u|^{2} \mathrm{~d} x \mathrm{~d} t \leq C_{24} R^{-1+\varepsilon}
$$

Proof. The proof follows the proof of [18, Lemma IX.8.2]. We put

$$
\mathcal{G}(R):=\frac{1}{\mathcal{T}} \int_{0}^{\mathcal{T}} \int_{\mathrm{B}^{R}}|\nabla u|^{2} \mathrm{~d} x \mathrm{~d} t=\frac{1}{\mathcal{T}} \int_{0}^{\mathcal{T}} \int_{R}^{\infty} \int_{\partial \mathrm{B}_{r}}|\nabla u|^{2} \mathrm{~d} S \mathrm{~d} r \mathrm{~d} t .
$$

By assumption $\nabla u \in C(G)$, whence $\mathcal{G} \in C^{1}(0, \infty)$. Multiplying both sides in (5.4) by $u$ and subsequently integrating over $\mathrm{B}_{R^{*}, R} \times[0, \mathcal{T}]$, we obtain

$$
\frac{1}{\mathcal{T}} \int_{0}^{\mathcal{T}} \int_{\mathrm{B}_{R^{*}, R}}|\nabla u|^{2} \mathrm{~d} x \mathrm{~d} t=\frac{1}{\mathcal{T}} \int_{0}^{\mathcal{T}} \int_{\partial \mathrm{B}_{R^{*}, R}} \frac{\lambda}{2}|u|^{2} n_{1}-\frac{1}{2}|u|^{2} u \cdot n+u \cdot(\nabla u \cdot n)-\mathfrak{p} u \cdot n \mathrm{~d} S \mathrm{~d} t .
$$

From the assumptions in (5.5) it follows that the function

$$
r \rightarrow \frac{1}{\mathcal{T}} \int_{0}^{\mathcal{T}} \int_{\partial \mathrm{B}_{r}}|u|^{3}+\left.\left|-\frac{1}{2}\right| u\right|^{2} u \cdot n+u \cdot(\nabla u \cdot n)-\mathfrak{p} u \cdot n \mid \mathrm{d} S \mathrm{~d} t
$$

is summable, that is, it belongs to $L^{1}((1, \infty))$. Consequently, there is a sequence $\left\{R_{k}\right\}_{k=1}^{\infty} \subset(0, \infty)$ with $\lim _{k \rightarrow \infty} R_{k}=\infty$ and the property

$$
\lim _{k \rightarrow \infty} R_{k} \frac{1}{\mathcal{T}} \int_{0}^{\mathcal{T}} \int_{\partial \mathrm{B}_{R_{k}}}|u|^{3}+\left.\left|-\frac{1}{2}\right| u\right|^{2} u \cdot n+u \cdot(\nabla u \cdot n)-\mathfrak{p} u \cdot n \mid \mathrm{d} S \mathrm{~d} t=0 .
$$

Employing Hölder's inequality, we observe that

$$
\frac{1}{\mathcal{T}} \int_{0}^{\mathcal{T}} \int_{\partial \mathrm{B}_{R}}|u|^{2} \mathrm{~d} S \mathrm{~d} t \leq c_{1} \frac{1}{\mathcal{T}} \int_{0}^{\mathcal{T}}\left(\int_{\partial \mathrm{B}_{R}}|u|^{3} \mathrm{~d} S\right)^{\frac{2}{3}} R^{\frac{2}{3}} \mathrm{~d} t \leq c_{1}\left(R \frac{1}{\mathcal{T}} \int_{0}^{\mathcal{T}} \int_{\partial \mathrm{B}_{R}}|u|^{3} \mathrm{~d} S \mathrm{~d} t\right)^{\frac{2}{3}} .
$$


It follows that

$$
\lim _{k \rightarrow \infty} \frac{1}{\mathcal{T}} \int_{0}^{\mathcal{T}} \int_{\partial \mathrm{B}_{R_{k}}} \frac{\lambda}{2}|u|^{2} n_{1}-\frac{1}{2}|u|^{2} u \cdot n+u \cdot(\nabla u \cdot n)-\mathfrak{p} u \cdot n \mathrm{~d} S \mathrm{~d} t=0 .
$$

Put

$$
F(R):=\frac{1}{\mathcal{T}} \int_{0}^{\mathcal{T}} \int_{\partial \mathrm{B}_{R}} \frac{\lambda}{2}|u|^{2} n_{1}-\frac{1}{2}|u|^{2} u \cdot n+u \cdot(\nabla u \cdot n)-\mathfrak{p} u \cdot n \mathrm{~d} S \mathrm{~d} t .
$$

Choosing $R^{*}=R_{k}$ in (5.7) and letting $k \rightarrow \infty$, we see that

$$
\mathcal{G}(R)=F(R) .
$$

By a similar estimate as in $(5.9)$, we find for $q \in(1, \infty)$

$$
\frac{1}{\mathcal{T}} \int_{0}^{\mathcal{T}} \int_{\partial \mathrm{B}_{R}}|u|^{2} \mathrm{~d} S \mathrm{~d} t \leq c_{2} R^{\frac{2(q-1)}{q}}\left(\frac{1}{\mathcal{T}} \int_{0}^{\mathcal{T}} \int_{\partial \mathrm{B}_{R}}|u|^{2 q} \mathrm{~d} S \mathrm{~d} t\right)^{\frac{1}{q}} .
$$

Employing Young's inequality, we then deduce

$$
R^{-\varepsilon} \frac{1}{\mathcal{T}} \int_{0}^{\mathcal{T}} \int_{\partial \mathrm{B}_{R}}|u|^{2} \mathrm{~d} S \mathrm{~d} t \leq c_{3}\left(R^{2-\varepsilon \frac{q}{q-1}}+\frac{1}{\mathcal{T}} \int_{0}^{\mathcal{T}} \int_{\partial \mathrm{B}_{R}}|u|^{2 q} \mathrm{~d} S \mathrm{~d} t\right) .
$$

Now fix $q \in\left(1, \frac{3}{3-\varepsilon}\right)$. Then $2-\varepsilon \frac{q}{q-1}<-1$. Moreover, by assumption (5.5), $u \in L^{2 q}(G)^{3}$. Thus

$$
R \rightarrow R^{-\varepsilon} \frac{1}{\mathcal{T}} \int_{0}^{\mathcal{T}} \int_{\partial \mathrm{B}_{R}}|u|^{2} \mathrm{~d} S \mathrm{~d} t \in L^{1}((1, \infty)) .
$$

This information together with the summability of the function in (5.8) implies that $R \rightarrow R^{-\varepsilon} F(R) \in L^{1}((1, \infty))$. Recalling (5.10), we deduce

$$
R \rightarrow R^{-\varepsilon} \mathcal{G}(R) \in L^{1}((1, \infty)) .
$$

Combining (5.12) with the fact that

$$
\mathcal{G}^{\prime}(R)=-\frac{1}{\mathcal{T}} \int_{0}^{\mathcal{T}} \int_{\partial \mathrm{B}_{R}}|\nabla u|^{2} \mathrm{~d} S \mathrm{~d} t \leq 0,
$$

we finally conclude, by $[18$, Lemma IX.8.1], that

$$
\mathcal{G}(R) R^{1-\varepsilon} \leq c_{4},
$$

which yields (5.6) 


\section{Asymptotic structure}

We observe in the following corollary that the decay property established for $u$ in Lemma 5.1.1 also holds for $\mathcal{P} u$ and $\mathcal{P}_{\perp} u$ separately.

Corollary 5.1.2. Under the same assumptions as in Lemma 5.1.1, it holds that

$$
\forall \varepsilon>0: \quad \frac{1}{\mathcal{T}} \int_{0}^{\mathcal{T}} \int_{\mathrm{B}^{R}}\left|\nabla \mathcal{P}_{\perp} u\right|^{2} \mathrm{~d} x \mathrm{~d} t+\int_{\mathrm{B}^{R}}|\nabla \mathcal{P} u|^{2} \mathrm{~d} x \leq C_{25} R^{-1+\varepsilon} .
$$

Proof. We simply observe that

$$
\begin{aligned}
& \frac{1}{\mathcal{T}} \int_{0}^{\mathcal{T}} \int_{\mathrm{B}^{R}}|\nabla u|^{2} \mathrm{~d} x \mathrm{~d} t \\
& \quad=\frac{1}{\mathcal{T}} \int_{0} \int_{\mathrm{B}^{R}}|\nabla \mathcal{P} \perp u|^{2} \mathrm{~d} x \mathrm{~d} t+\int_{\mathrm{B}^{R}}|\nabla \mathcal{P} u|^{2} \mathrm{~d} x+2 \frac{1}{\mathcal{T}} \int_{0}^{\mathcal{T}} \int_{\mathrm{B}^{R}} \nabla \mathcal{P} u: \nabla \mathcal{P} \perp u \mathrm{~d} x \mathrm{~d} t
\end{aligned}
$$

and

$$
\frac{1}{\mathcal{T}} \int_{0}^{\mathcal{T}} \int_{\mathrm{B}^{R}} \nabla \mathcal{P} u: \nabla \mathcal{P}_{\perp} u \mathrm{~d} x \mathrm{~d} t=\int_{\mathrm{B}^{R}} \nabla \mathcal{P} u:\left(\frac{1}{\mathcal{T}} \int_{0}^{\mathcal{T}} \nabla \mathcal{P}_{\perp} u \mathrm{~d} t\right) \mathrm{d} x=0 .
$$

We will utilize the representation formula introduced in Section 3.3 to establish the desired asymptotic expansion of a solution $u$ to (5.4). More specifically, we shall express $u$ in terms of a fundamental solution to the corresponding linearized problem, that is, a solution $(\Gamma, \Pi) \in \mathscr{S}^{\prime}(G)^{3 \times 3} \times \mathscr{S}^{\prime}(G)^{3}$ to

$$
\begin{cases}\partial_{t} \Gamma_{j l}-\Delta \Gamma_{j l}-\lambda \partial_{1} \Gamma_{j l}+\partial_{j} \Pi_{l}=\delta_{j l} \delta & \text { in } G, \\ \partial_{j} \Gamma_{j l}=0 & \text { in } G .\end{cases}
$$

Here, $\delta_{l j}$ denotes the Kronecker delta, and $\delta$ the delta distribution on $G$. In (3.28) we have already encountered an expression, given in terms of a Fourier multiplier, of the fundamental solution $\Gamma_{j l}$.

In the following lemmas we shall derive a point-wise estimate of $\Gamma_{j l}$. We will use the time-averaging projection introduced in Section 3.5 to decompose $\Gamma=\mathcal{P} \Gamma+\mathcal{P}_{\perp} \Gamma$ and estimate $\mathcal{P} \Gamma$ and $\mathcal{P}_{\perp} \Gamma$ separately. For this purpose, we need the following lemma.

Lemma 5.1.3. Let $\lambda>0, k \in \mathbb{Z}$, and

$$
\Gamma_{\mathrm{SOR}}^{\lambda, k}: \mathbb{R}^{3} \backslash\{0\} \rightarrow \mathbb{C}, \quad \Gamma_{\mathrm{SOR}}^{\lambda, k}(x):=\frac{1}{4 \pi|x|} \mathrm{e}^{-\left(i \frac{2 \pi}{\mathcal{T}} k+\left(\frac{\lambda}{2}\right)^{2}\right)^{\frac{1}{2}}|x|-\frac{\lambda}{2} x_{1}} .
$$


Then

$$
\left|\Gamma_{\mathrm{SOR}}^{\lambda, k}(x)\right| \leq \frac{1}{4 \pi|x|} \mathrm{e}^{-C_{26}|k|^{\frac{1}{2}}|x|}
$$

with $C_{26}=C_{26}(\lambda, \mathcal{T})$. Moreover, for $q \in[1, \infty)$

$$
\left(\sum_{k \in \mathbb{Z} \backslash\{0\}}\left|\Gamma_{\mathrm{SOR}}^{\lambda, k}(x)\right|^{q}\right)^{\frac{1}{q}} \leq C_{27}|x|^{-\left(1+\frac{2}{q}\right)} \mathrm{e}^{-C_{28}|x|}
$$

with $C_{27}=C_{27}(q, \lambda, \mathcal{T})$ and $C_{28}=C_{28}(q, \lambda, \mathcal{T})>0$.

Proof. We first observe that

$$
\begin{aligned}
\operatorname{Re}\left[\left(i \frac{2 \pi}{\mathcal{T}}\right.\right. & \left.\left.k+(\lambda / 2)^{2}\right)^{\frac{1}{2}}\right]=\left|i \frac{2 \pi}{\mathcal{T}} k+(\lambda / 2)^{2}\right|^{\frac{1}{2}} \cos \left(\frac{1}{2} \arctan \left(\frac{\frac{2 \pi}{\mathcal{T}} k}{(\lambda / 2)^{2}}\right)\right) \\
= & \left(\left(\frac{2 \pi}{\mathcal{T}}\right)^{2} k^{2}+(\lambda / 2)^{4}\right)^{\frac{1}{4}} \frac{1}{\sqrt{2}}\left(1+\cos \left(\arctan \left(\frac{\frac{2 \pi}{\mathcal{T}} k}{(\lambda / 2)^{2}}\right)\right)\right)^{\frac{1}{2}} \\
= & \left(\left(\frac{2 \pi}{\mathcal{T}}\right)^{2} k^{2}+(\lambda / 2)^{4}\right)^{\frac{1}{4}} \frac{1}{\sqrt{2}}\left(1+\left(1+\frac{\left(\frac{2 \pi}{\mathcal{T}}\right)^{2} k^{2}}{(\lambda / 2)^{4}}\right)^{-\frac{1}{2}}\right)^{\frac{1}{2}} \\
= & \frac{1}{\sqrt{2}}(\lambda / 2)\left(\left(1+\frac{\left(\frac{2 \pi}{\mathcal{T}}\right)^{2} k^{2}}{(\lambda / 2)^{4}}\right)^{\frac{1}{2}}+1\right)^{\frac{1}{2}} .
\end{aligned}
$$

It follows that

$$
\forall k \in \mathbb{Z} \backslash\{0\}: \operatorname{Re}\left[\left(i \frac{2 \pi}{\mathcal{T}} k+(\lambda / 2)^{2}\right)^{\frac{1}{2}}-(\lambda / 2)\right]>0
$$

and

$$
\lim _{|k| \rightarrow \infty} \frac{\operatorname{Re}\left[\left(i \frac{2 \pi}{\mathcal{T}} k+(\lambda / 2)^{2}\right)^{\frac{1}{2}}-(\lambda / 2)\right]}{|k|^{\frac{1}{2}}}=\sqrt{\frac{\frac{2 \pi}{\mathcal{T}}}{2}} .
$$

Consequently, there is a constant $c_{1}=c_{1}(\lambda, \mathcal{T})>0$ such that

$$
\forall k \in \mathbb{Z} \backslash\{0\}: \operatorname{Re}\left[\left(i \frac{2 \pi}{\mathcal{T}} k+(\lambda / 2)^{2}\right)^{\frac{1}{2}}-(\lambda / 2)\right] \geq c_{1}|k|^{\frac{1}{2}},
$$

from which we conclude that

$$
\begin{aligned}
\operatorname{Re}[ & \left.-\left(i \frac{2 \pi}{\mathcal{T}} k+(\lambda / 2)^{2}\right)^{\frac{1}{2}}|x|-(\lambda / 2) x_{1}\right] \\
& \leq-\operatorname{Re}\left[\left(i \frac{2 \pi}{\mathcal{T}} k+(\lambda / 2)^{2}\right)^{\frac{1}{2}}-(\lambda / 2)\right]|x| \leq-c_{1}|k|^{\frac{1}{2}}|x| .
\end{aligned}
$$


5 Asymptotic structure

We can now estimate

$$
\begin{aligned}
\left|\Gamma_{\text {SOR }}^{\lambda, k}(x)\right| & \leq \frac{1}{4 \pi|x|} \mathrm{e}^{\operatorname{Re}\left[-\left(i \frac{2 \pi}{\mathcal{T}} k+(\lambda / 2)^{2}\right)^{\frac{1}{2}}|x|-(\lambda / 2) x_{1}\right]} \\
& \leq \frac{1}{4 \pi|x|} \mathrm{e}^{-c_{1}|k|^{\frac{1}{2}}|x|}
\end{aligned}
$$

and conclude (5.16). Estimate (5.18) further implies

$$
\begin{aligned}
\sum_{k \in \mathbb{Z} \backslash\{0\}}\left|\Gamma_{\mathrm{SOR}}^{\lambda, k}(x)\right|^{q} & \leq c_{2}|x|^{-q} \sum_{k \in \mathbb{Z} \backslash\{0\}} \mathrm{e}^{-q c_{1}|k|^{\frac{1}{2}}|x|} \\
& \leq c_{3}|x|^{-q} \sum_{j=1}^{\infty} \sum_{k=j^{2}}^{(j+1)^{2}-1} \mathrm{e}^{-q c_{1} j|x|} \\
& \leq c_{4}|x|^{-q} \sum_{j=1}^{\infty} j \mathrm{e}^{-q c_{1} j|x|} \\
& =c_{4}|x|^{-q} \frac{\mathrm{e}^{-q c_{1}|x|}}{\left(1-\mathrm{e}^{-q c_{1}|x|}\right)^{2}} \\
& \leq c_{5}|x|^{-q}|x|^{-2} \mathrm{e}^{-\frac{1}{2} q c_{1}|x|}
\end{aligned}
$$

with $c_{5}=c_{5}(q, \lambda, \mathcal{T})$. We have thus shown (5.17).

We can now establish a pointwise estimate of $\mathcal{P}_{\perp} \Gamma$.

Lemma 5.1.4. Let $\lambda>0$ and define

$$
\Gamma^{\perp} \in \mathscr{S}^{\prime}(G)^{3 \times 3}, \quad \Gamma^{\perp}:=\mathcal{F}_{G}^{-1}\left[\frac{1-\kappa_{0}(\xi, k)}{|\xi|^{2}+i\left(\frac{2 \pi}{\mathcal{T}} k-\lambda \xi_{1}\right)}\left(I-\frac{\xi \otimes \xi}{|\xi|^{2}}\right)\right] .
$$

Then

$$
\Psi: \mathscr{S}(G)^{3} \rightarrow \mathscr{S}^{\prime}(G)^{3}, \quad \Psi_{j}(f):=\Gamma_{j l}^{\perp} * f_{l}
$$

extends uniquely for any $q \in(1, \infty)$ to a bounded linear operator

$$
\Psi: L^{q}(G)^{3} \rightarrow L^{q}(G)^{3} .
$$

Moreover,

$$
\forall|x| \geq 2: \quad\left(\frac{1}{\mathcal{T}} \int_{0}^{\mathcal{T}}\left|\Gamma_{j l}^{\perp}(x, t)\right|^{2} \mathrm{~d} t\right)^{\frac{1}{2}} \leq \frac{C_{29}}{|x|^{3}},
$$

with $C_{29}=C_{29}(\lambda, \mathcal{T})$, and

$$
\forall r \in[1,3 / 2): \quad \Gamma_{j l}^{\perp} \in L^{r}(G) .
$$


Proof. Recalling the proof of Theorem 3.6.5, in particular definition (3.64) of the multiplier $M: \widehat{G} \rightarrow \mathbb{C}$, and definition (3.19) of the Helmholtz decomposition, we recognize

$$
\Psi(f)=\mathcal{F}_{G}^{-1}\left[M \widehat{\mathcal{P}_{H} f}\right] .
$$

In the proof of Theorem 3.6.5 we already showed that $M$ is an $L^{q}(G)$ multiplier. In view of Lemma 3.2.3, we thus conclude (5.21).

Now consider $k \in \mathbb{Z} \backslash\{0\}$. Put

$$
\Gamma_{j l}^{k}:=\mathcal{F}_{\mathbb{R}^{3}}^{-1}\left[\frac{\xi_{j} \xi_{l}}{|\xi|^{2}} \cdot \frac{1}{|\xi|^{2}+i\left(\frac{2 \pi}{\mathcal{T}} k-\lambda \xi_{1}\right)}\right] \in \mathscr{S}^{\prime}\left(\mathbb{R}^{3}\right) .
$$

A direct computation yields

$$
\Gamma_{j l}^{k}=\partial_{j} \partial_{l} \mathcal{F}_{\mathbb{R}^{3}}^{-1}\left[\mathcal{F}_{\mathbb{R}^{3}}\left(\Gamma_{\mathrm{L}}\right) \cdot \mathcal{F}_{\mathbb{R}^{3}}\left(\Gamma_{\mathrm{SOR}}^{\lambda, k}\right)\right]
$$

with

$$
\Gamma_{\mathrm{L}}: \mathbb{R}^{3} \backslash\{0\} \rightarrow \mathbb{C}, \quad \Gamma_{\mathrm{L}}(x):=\frac{1}{4 \pi|x|}
$$

and $\Gamma_{\mathrm{SOR}}^{\lambda, k}$ given by (5.15). In view of (5.16), we see that both $\Gamma_{\mathrm{L}}$ and $\Gamma_{\mathrm{SOR}}^{\lambda, k}$ are "regular" enough to express the right-hand side of (5.24) in terms of a classical convolution integral. More precisely, we have

$$
\Gamma_{j l}^{k}(x)=\partial_{j} \partial_{l} \int_{\mathbb{R}^{3}} \Gamma_{\mathrm{L}}(x-y) \Gamma_{\mathrm{SOR}}^{\lambda, k}(y) \mathrm{d} y .
$$

We introduce at this point a cut-off function $\chi \in C_{0}^{\infty}(\mathbb{R} ; \mathbb{R})$ with

$$
\chi(x)=\left\{\begin{array}{ll}
0 & \text { if } 0 \leq|x| \leq \frac{1}{2} \\
1 & \text { if } 1 \leq|x| \leq 3
\end{array} \text { or } 4 \leq|x|,\right.
$$

and define for $R>0$

$$
\chi_{R} \in C_{0}^{\infty}\left(\mathbb{R}^{3} ; \mathbb{R}\right), \quad \chi_{R}(x):=\chi(|x| / R) .
$$

Now consider an $x \in \mathbb{R}^{3}$ with $|x|>2$. Put $R:=\frac{|x|}{2}$. Note that this implies $x \in \mathrm{B}_{3 R, R}$. We use $\chi_{R}$ to split the convolution integral in (5.25) as follows:

$$
\begin{aligned}
\Gamma_{j l}^{k}(x)= & \partial_{j} \partial_{l} \int_{\mathbb{R}^{3}} \Gamma_{\mathrm{L}}(x-y) \Gamma_{\mathrm{SOR}}^{\lambda, k}(y) \chi_{R}(y) \mathrm{d} y+\partial_{j} \partial_{l} \int_{\mathbb{R}^{3}} \Gamma_{\mathrm{L}}(x-y) \Gamma_{\mathrm{SOR}}^{\lambda, k}(y)\left(1-\chi_{R}(y)\right) \mathrm{d} y \\
= & \partial_{j} \partial_{l} \int_{\mathrm{B}_{4 R, R / 2}} \Gamma_{\mathrm{L}}(x-y) \Gamma_{\mathrm{SOR}}^{\lambda, k}(y) \chi_{R}(y) \mathrm{d} y \\
& +\partial_{j} \partial_{l} \int_{\mathrm{B}^{3 R}} \Gamma_{\mathrm{L}}(x-y) \Gamma_{\mathrm{SOR}}^{\lambda, k}(y)\left(1-\chi_{R}(y)\right) \mathrm{d} y \\
& +\partial_{j} \partial_{l} \int_{\mathrm{B}_{R}} \Gamma_{\mathrm{L}}(x-y) \Gamma_{\mathrm{SOR}}^{\lambda, k}(y)\left(1-\chi_{R}(y)\right) \mathrm{d} y=: I_{1}(x)+I_{2}(x)+I_{3}(x) .
\end{aligned}
$$




\section{Asymptotic structure}

We can estimate

$$
\left|I_{1}(x)\right| \leq \int_{\mathrm{B}_{4 R, R / 2}} \frac{1}{4 \pi|x-y|^{2}}\left|\partial_{j}\left[\Gamma_{\mathrm{SOR}}^{\lambda, k}(y) \chi_{R}(y)\right]\right| \mathrm{d} y .
$$

After computing $\partial_{j}\left[\Gamma_{\mathrm{SOR}}^{\lambda, k}(y)\right]$, we can estimate, in view of (5.16),

$$
\left|\partial_{j}\left[\Gamma_{\mathrm{SOR}}^{\lambda, k}(y)\right]\right| \leq c_{1}\left(\frac{1}{|y|^{2}}+\frac{|k|^{\frac{1}{2}}}{|y|}\right) \mathrm{e}^{-C_{26}|k|^{\frac{1}{2}}|y|} .
$$

Hence, for $y \in \mathrm{B}_{4 R, R / 2}$ and arbitrary $h \in \mathbb{N}_{0}$

$$
\left|\partial_{j}\left[\Gamma_{\mathrm{SOR}}^{\lambda, k}(y)\right]\right| \leq c_{2} \frac{1}{|k|^{(h-1) / 2} R^{h+2}},
$$

with $c_{2}=c_{2}(h, \lambda, \mathcal{T})$. Similarly, we see for $y \in \mathrm{B}_{4 R, R / 2}$ that

$$
\left|\Gamma_{\mathrm{SOR}}^{\lambda, k}(y)\right| \leq c_{3} \frac{1}{|k|^{h / 2} R^{h+1}},
$$

with $c_{3}=c_{3}(h, \lambda, \mathcal{T})$. It now follows from (5.26) that

$$
\left|I_{1}(x)\right| \leq c_{4} \frac{1}{|k|^{(h-1) / 2} R^{h}} .
$$

Choosing $h=3$, we thus obtain

$$
\left|I_{1}(x)\right| \leq c_{5} \frac{1}{|k| R^{3}},
$$

with $c_{5}=c_{5}(\lambda, \mathcal{T})$. Similarly, we estimate

$$
\begin{aligned}
\left|I_{2}(x)\right| & \leq c_{6} \int_{\mathrm{B}^{3 R}} \frac{1}{|x-y|^{3}}\left|\Gamma_{\mathrm{SOR}}^{\lambda, k}(y)\right| \mathrm{d} y \\
& \leq c_{7} \int_{\mathrm{B}^{3 R}} \frac{1}{|x-y|^{3}} \frac{1}{|y|} \mathrm{e}^{-C_{26}|k|^{\frac{1}{2}}|y|} \mathrm{d} y \\
& \leq c_{8} \int_{\mathrm{B}^{3 R}} \frac{1}{R^{3}} \frac{1}{|y|} \frac{1}{|k|^{\frac{3}{2}}|y|^{3}} \mathrm{~d} y \\
& \leq c_{9} \frac{1}{|k| R^{3}}
\end{aligned}
$$


with $c_{9}=c_{9}(\lambda, \mathcal{T})$, and

$$
\begin{aligned}
\left|I_{3}(x)\right| & \leq c_{10} \int_{\mathrm{B}_{R}} \frac{1}{|x-y|^{3}}\left|\Gamma_{\mathrm{SOR}}^{\lambda, k}(y)\right| \mathrm{d} y \\
& \leq c_{11} \int_{\mathrm{B}_{R}} \frac{1}{R^{3}} \frac{1}{|y|} \mathrm{e}^{-C_{26}|k|^{\frac{1}{2}}|y|} \mathrm{d} y \\
& =c_{12} \frac{1}{R^{3}} \int_{0}^{R} r \mathrm{e}^{-C_{26}|k|^{\frac{1}{2}} r} \mathrm{~d} r \\
& \leq c_{12} \frac{1}{|k| R^{3}} \int_{0}^{\infty} s \mathrm{e}^{-C_{26} s} \mathrm{~d} s \\
& =c_{13} \frac{1}{|k| R^{3}},
\end{aligned}
$$

with $c_{13}=c_{13}(\lambda, \mathcal{T})$. Combining (5.27), (5.28), and (5.29), we deduce

$$
\left|\Gamma_{j l}^{k}(x)\right| \leq c_{14} \frac{1}{|k||x|^{3}},
$$

with $c_{14}=c_{14}(\lambda, \mathcal{T})$. By Plancherel's identity, it follows that ${ }^{1}$

$$
\begin{aligned}
\left(\frac{1}{\mathcal{T}} \int_{0}^{\mathcal{T}}\left|\Gamma_{j l}^{\perp}(x, t)\right|^{2} \mathrm{~d} t\right)^{\frac{1}{2}} & =\left(\frac{1}{\mathcal{T}} \int_{0}^{\mathcal{T}}\left|\mathcal{F}_{\mathbb{R} / \mathcal{T} \mathbb{Z}}^{-1}\left[\left(1-\kappa_{0}(k)\right)\left(\delta_{j l} \Gamma_{h h}^{k}(x)-\Gamma_{j l}^{k}(x)\right)\right]\right|^{2} \mathrm{~d} t\right)^{\frac{1}{2}} \\
& =\left\|\left(1-\kappa_{0}(k)\right)\left(\delta_{j l} \Gamma_{h h}^{k}(x)-\Gamma_{j l}^{k}(x)\right)\right\|_{\ell^{2}(\mathbb{Z})} \\
& \leq c_{15}\left(\sum_{k \in \mathbb{Z} \backslash\{0\}} \frac{1}{\left(|k||x|^{3}\right)^{2}}\right)^{\frac{1}{2}} \\
& \leq c_{16} \frac{1}{|x|^{3}}
\end{aligned}
$$

which yields (5.22).

It remains to show (5.23). For this purpose, we recall that the Riesz transform

$$
\mathfrak{R}_{j}: \mathscr{S}\left(\mathbb{R}^{3}\right) \rightarrow \mathscr{S}^{\prime}\left(\mathbb{R}^{3}\right), \quad \mathfrak{R}_{j}(f):=\mathcal{F}_{\mathbb{R}^{3}}^{-1}\left[\frac{\xi_{j}}{|\xi|} \widehat{f}\right]
$$

extends for all $q \in(1, \infty)$ to a bounded linear operator

$$
\mathfrak{R}_{j}: L^{q}\left(\mathbb{R}^{3}\right) \rightarrow L^{q}\left(\mathbb{R}^{3}\right) ;
$$

\footnotetext{
${ }^{1}$ Recall that we make use of Einstein's summation convention and implicitly sum over all repeated indices.
} 


\section{Asymptotic structure}

see for example [28, Corollary 4.2.8]. Observe that

$$
\Gamma_{j l}^{\perp}=\left[\delta_{j l}\left(\mathfrak{R}_{h} \circ \mathfrak{R}_{h}\right)-\mathfrak{R}_{j} \circ \mathfrak{R}_{l}\right]\left(\mathcal{F}_{\mathbb{R} / \mathcal{T} \mathbb{Z}}^{-1}\left[\left(1-\kappa_{0}(k)\right) \Gamma_{\mathrm{SOR}}^{\lambda, k}\right]\right) .
$$

It follows that

$$
\frac{1}{\mathcal{T}} \int_{0}^{\mathcal{T}} \int_{\mathbb{R}^{3}}\left|\Gamma_{j l}^{\perp}\right|^{r} \mathrm{~d} x \mathrm{~d} t \leq c_{17} \frac{1}{\mathcal{T}} \int_{0}^{\mathcal{T}} \int_{\mathbb{R}^{3}}\left|\mathcal{F}_{\mathbb{R} / \mathcal{T} \mathbb{Z}}^{-1}\left[\left(1-\kappa_{0}(k)\right) \Gamma_{\mathrm{SOR}}^{\lambda, k}\right]\right|^{r} \mathrm{~d} x \mathrm{~d} t .
$$

If $r \in(1,2)$ we can employ Hölder's inequality to obtain

$$
\frac{1}{\mathcal{T}} \int_{0}^{\mathcal{T}} \int_{\mathbb{R}^{3}}\left|\Gamma_{j l}^{\perp}\right|^{r} \mathrm{~d} x \mathrm{~d} t \leq c_{18} \int_{\mathbb{R}^{3}}\left(\frac{1}{\mathcal{T}} \int_{0}^{\mathcal{T}}\left|\mathcal{F}_{\mathbb{R} / \mathcal{T} \mathbb{Z}}^{-1}\left[\left(1-\kappa_{0}(k)\right) \Gamma_{\text {SOR }}^{\lambda, k}\right]\right|^{2} \mathrm{~d} t\right)^{\frac{r}{2}} \mathrm{~d} x .
$$

By Plancherel's theorem, we then find that

$$
\frac{1}{\mathcal{T}} \int_{0}^{\mathcal{T}} \int_{\mathbb{R}^{3}}\left|\Gamma_{j l}^{\perp}\right|^{r} \mathrm{~d} x \mathrm{~d} t \leq c_{19} \int_{\mathbb{R}^{3}}\left(\sum_{k \in \mathbb{Z} \backslash\{0\}}\left|\Gamma_{\mathrm{SOR}}^{\lambda, k}\right|^{2}\right)^{\frac{r}{2}} \mathrm{~d} x .
$$

Thus, (5.17) implies

$$
\frac{1}{\mathcal{T}} \int_{0}^{\mathcal{T}} \int_{\mathbb{R}^{3}}\left|\Gamma_{j l}^{\perp}\right|^{r} \mathrm{~d} x \mathrm{~d} t \leq c_{20} \int_{\mathbb{R}^{3}}\left(|x|^{-2} \mathrm{e}^{-C_{28}|x|}\right)^{r} \mathrm{~d} x
$$

from which (5.23) follows.

We are now in a position to establish the desired asymptotic expansion of a solution to (5.4). The asymptotic profile in the expansion will be given in terms of the classical Oseen fundamental solution

$$
\begin{aligned}
& \Gamma_{\mathrm{O}}^{\lambda}: \mathbb{R}^{3} \backslash\{0\} \rightarrow \mathbb{R}^{3 \times 3}, \quad\left[\Gamma_{\mathrm{O}}^{\lambda}(x)\right]_{i j}:=\left(\delta_{i j} \Delta-\partial_{i} \partial_{j}\right) \Phi^{\lambda}(x), \\
& \Phi^{\lambda}(x):=\frac{1}{4 \pi \lambda} \int_{0}^{\lambda\left(|x|+x_{1}\right) / 2} \frac{1-\mathrm{e}^{-\tau}}{\tau} \mathrm{d} \tau .
\end{aligned}
$$

See also [17, Chapter VII.3] for a closed-form expression of $\Gamma_{\mathrm{O}}^{\lambda}$. The Oseen fundamental solution solves, in terms of distributions,

$$
\begin{cases}-\Delta \Gamma_{\mathrm{O} i j}^{\lambda}+\partial_{i} \partial_{j} \Gamma_{\mathrm{L}}-\lambda \partial_{1} \Gamma_{\mathrm{O} i j}^{\lambda}=\delta_{i j} \delta & \text { in } \mathbb{R}^{3} \\ \partial_{i} \Gamma_{\mathrm{O} i j}^{\lambda}=0 & \text { in } \mathbb{R}^{3}\end{cases}
$$

We recall the following well-known properties of $\Gamma_{\mathrm{O}}^{\lambda}$ : 
Lemma 5.1.5. Let $\lambda>0$. The Oseen fundamental solution $\Gamma_{\mathrm{O}}^{\lambda}$ satisfies

$$
\begin{array}{ll}
\forall|x|>0: & \left|\Gamma_{\mathrm{O}}^{\lambda}(x)\right| \leq C_{30}|x|^{-1}, \\
\forall|x|>1: & \left|\nabla \Gamma_{\mathrm{O}}^{\lambda}(x)\right| \leq C_{31}|x|^{-\frac{3}{2}}, \\
\forall r>0: & \int_{\partial \mathrm{B}_{r}}\left|\nabla \Gamma_{\mathrm{O}}^{\lambda}(x)\right| \mathrm{d} S \leq C_{32} r^{-\frac{1}{2}} .
\end{array}
$$

Moreover, $\Gamma_{\mathrm{O}}^{\lambda}$ enjoys the summability properties

$$
\begin{array}{ll}
\forall q \in(2, \infty): & \Gamma_{\mathrm{O}}^{\lambda} \in L^{q}\left(\mathbb{R}^{3} \backslash \mathrm{B}_{r}\right)^{3 \times 3} \text { for any } r>0, \\
\forall q \in[1,3): & \Gamma_{\mathrm{O}}^{\lambda} \in L_{\text {loc }}^{q}\left(\mathbb{R}^{3}\right)^{3 \times 3}, \\
\forall q \in(4 / 3, \infty): & \nabla \Gamma_{\mathrm{O}}^{\lambda} \in L^{q}\left(\mathbb{R}^{3} \backslash \mathrm{B}_{r}\right)^{3 \times 3 \times 3} \text { for any } r>0, \\
\forall q \in[1,3 / 2): & \nabla \Gamma_{\mathrm{O}}^{\lambda} \in L_{\text {loc }}^{q}\left(\mathbb{R}^{3}\right)^{3 \times 3 \times 3} .
\end{array}
$$

Proof. We refer to [17, Chapter VII: (3.24), (3.28), (3.32), (3.33)] for (5.32), (5.35), (5.33), and (5.37). Estimate (5.34) follows from [17, Exercise VII.3.1]. Finally, (5.36) and (5.38) are direct consequences of (5.32) and (5.34), respectively.

We can now state and prove the main theorem of the chapter.

Theorem 5.1.6. Let $\lambda>0, f \in C_{0}^{\infty}(G)^{3}$, and $(u, \mathfrak{p})$ be a solution to (5.4) with $u=v+w$ satisfying for some $r \in(8, \infty)$ :

$$
(v, w, \mathfrak{p}) \in \mathrm{X}_{\sigma, \text { Oseen }}^{\frac{6}{5}, r}\left(\mathbb{R}^{3}\right) \times W_{\sigma, \perp}^{1,2, \frac{6}{5}, r}(G) \times \mathrm{X}_{\mathrm{P}}^{\frac{6}{5}, r}(G) .
$$

Then

$$
u(x, t)=\Gamma_{\mathrm{O}}^{\lambda}(x) \cdot\left(\frac{1}{\mathcal{T}} \int_{0}^{\mathcal{T}} \int_{\mathbb{R}^{3}} f \mathrm{~d} x \mathrm{~d} t\right)+\mathscr{R}(x, t)
$$

with

$$
\forall \varepsilon>0: \quad|\mathscr{R}(x, t)| \leq C_{33}|x|^{-\frac{4}{3}+\varepsilon} \quad \text { for }|x| \geq 1,
$$

where $C_{33}=C_{33}(\varepsilon)$.

Proof. By Corollary 4.2.2, $u$ is a smooth vector field. Since $\Gamma_{\mathrm{O}}^{\lambda}$ is smooth away from the origin, it is enough to show the estimate in (5.41) for large $|x|$.

In view of the fact that $f \in C_{0}^{\infty}(G)^{3}$, it follows from the regularity obtained in Lemma 4.1.2, Theorem 4.2.1, and Lemma 4.2.3, together with the aid of classical Sobolev embedding theorems, that the solution $(u, \mathfrak{p})$ satisfies condition (5.5) in Lemma 5.1.1. Observe in particular that $v \in \mathrm{X}_{\sigma, \text { Oseen }}^{\frac{6}{5}, r}\left(\mathbb{R}^{3}\right)$ implies $v \in L^{3}\left(\mathbb{R}^{3}\right)^{3}$. Consequently, we have the estimate from Lemma 5.1.1 and thus also Corollary 5.1.2 at our disposal. 


\section{Asymptotic structure}

We will now utilize that $w$ satisfies

$$
\begin{cases}\partial_{t} w-\Delta w-\lambda \partial_{1} w=\mathcal{P}_{H} \mathcal{P}_{\perp} f-\mathcal{P}_{H}\left[\mathcal{P}_{\perp}[w \cdot \nabla w]+w \cdot \nabla v+v \cdot \nabla w\right] & \text { in } G \\ \operatorname{div} w=0 & \text { in } G .\end{cases}
$$

From Lemma 5.1.4 we known that convolution with the fundamental solution $\Gamma^{\perp}$ extends to a bounded operator on $L^{q}(G)^{3}$ for all $q \in(1, \infty)$. As in the proof of Theorem 4.1.5, one can verify that the right-hand side in $(5.42)$ belongs to $L^{\frac{6}{5}, r}(G)^{3}$. Thus, convolution of $\Gamma^{\perp}$ with the right-hand side in (5.42) is well-defined in a classical sense. Recalling the uniqueness statement of Lemma 3.6.4, we therefore obtain

$$
w=\Gamma^{\perp} *(f-w \cdot \nabla w-w \cdot \nabla v-v \cdot \nabla w)
$$

with componentwise convolution with respect to the variables in $G$. Put

$$
\begin{aligned}
& I_{1}(x, t):=\Gamma^{\perp} *[w \cdot \nabla w](x, t), \\
& I_{2}(x, t):=\Gamma^{\perp} *[w \cdot \nabla v](x, t), \\
& I_{3}(x, t):=\Gamma^{\perp} *[v \cdot \nabla w](x, t), \\
& I_{4}(x, t):=\Gamma^{\perp} * f(x, t) .
\end{aligned}
$$

We shall give a pointwise estimate of $I_{1}, I_{2}, I_{3}$, and $I_{4}$. Let $|x|=2 R$. We first split

$$
\begin{aligned}
I_{1}(x, t)= & \frac{1}{\mathcal{T}} \int_{0}^{\mathcal{T}} \int_{\mathrm{B}_{R}} \Gamma^{\perp}(x-y, t-s)[w \cdot \nabla w](y, s) \mathrm{d} y \mathrm{~d} s \\
& +\frac{1}{\mathcal{T}} \int_{0}^{\mathcal{T}} \int_{\mathrm{B}^{R}} \Gamma^{\perp}(x-y, t-s)[w \cdot \nabla w](y, s) \mathrm{d} y \mathrm{~d} s \\
= & : I_{11}+I_{12} .
\end{aligned}
$$

Employing Hölder's inequality and recalling (5.22), we deduce

$$
\begin{aligned}
\left|I_{11}\right| & \leq \int_{\mathrm{B}_{R}}\left(\frac{1}{\mathcal{T}} \int_{0}^{\mathcal{T}}\left|\Gamma^{\perp}(x-y, s)\right|^{2} \mathrm{~d} s\right)^{\frac{1}{2}}\left(\frac{1}{\mathcal{T}} \int_{0}^{\mathcal{T}}|w \cdot \nabla w|^{2} \mathrm{~d} s\right)^{\frac{1}{2}} \mathrm{~d} y \\
& \leq c_{1} \frac{1}{R^{3}}\left(\int_{\mathrm{B}_{R}} 1 \mathrm{~d} y\right)^{\frac{1}{2}}\left(\frac{1}{\mathcal{T}} \int_{0} \int_{\mathrm{B}_{R}}|w \cdot \nabla w|^{2} \mathrm{~d} y \mathrm{~d} s\right)^{\frac{1}{2}} \\
& \leq c_{2} R^{-\frac{3}{2}}
\end{aligned}
$$


where we in the last inequality use that $w \cdot \nabla w \in L^{2}(G)^{3}$. Consider $\alpha \in\left[1, \frac{3}{2}\right)$ and $\beta \in(1, \infty)$, and let $\alpha^{\prime}$ and $\beta^{\prime}$ denote the corresponding Hölder conjugates. We find, recalling again $(5.23)$, that

$$
\begin{aligned}
\left|I_{12}\right| & \leq\left(\frac{1}{\mathcal{T}} \int_{0}^{\mathcal{T}} \int_{\mathrm{B}^{R}}\left|\Gamma^{\perp}(x-y, s)\right|^{\alpha} \mathrm{d} y \mathrm{~d} s\right)^{\frac{1}{\alpha}}\left(\frac{1}{\mathcal{T}} \int_{0}^{\mathcal{T}} \int_{\mathrm{B}^{R}}|w|^{\alpha^{\prime}}|\nabla w|^{\alpha^{\prime}} \mathrm{d} y \mathrm{~d} s\right)^{\frac{1}{\alpha^{\prime}}} \\
& \leq c_{3}\left(\frac{1}{\mathcal{T}} \int_{0}^{\mathcal{T}} \int_{\mathrm{B}^{R}}|\nabla w|^{\frac{2}{\beta}}|\nabla w|^{\alpha^{\prime}-\frac{2}{\beta}} \mathrm{d} y \mathrm{~d} s\right)^{\frac{1}{\alpha^{\prime}}}\|w\|_{\infty, \mathrm{B}^{R}} \\
& \leq c_{4}\left(\frac{1}{\mathcal{T}} \int_{0}^{\mathcal{T}} \int_{\mathrm{B}^{R}}|\nabla w|^{2} \mathrm{~d} y \mathrm{~d} s\right)^{\frac{1}{\beta \alpha^{\prime}}}\left(\frac{1}{\mathcal{T}} \int_{0}^{\mathcal{T}} \int_{\mathrm{B}^{R}}|\nabla w|^{\beta^{\prime}\left(\alpha^{\prime}-\frac{2}{\beta}\right)} \mathrm{d} y \mathrm{~d} s\right)^{\frac{1}{\beta^{\prime} \alpha^{\prime}}}\|w\|_{\infty, \mathrm{B}^{R} .}
\end{aligned}
$$

Since

$$
\frac{1}{\beta \alpha^{\prime}} \rightarrow \frac{1}{3} \quad \text { and } \quad \beta^{\prime}\left(\alpha^{\prime}-\frac{2}{\beta}\right) \rightarrow \infty \quad \text { as }(\alpha, \beta) \rightarrow\left(\frac{3}{2}, 1\right),
$$

it follows, using Corollary 5.1.2 and recalling the summability of $\nabla w$ ensured by Theorem 4.2.1, that

$$
\forall \varepsilon>0: \quad\left|I_{12}\right| \leq c_{5} R^{-\frac{1}{3}+\varepsilon}\|w\|_{\infty, \mathrm{B}^{R}}
$$

We thus conclude

$$
\forall \varepsilon>0: \quad\left|I_{1}\right| \leq c_{6}\left(R^{-\frac{3}{2}}+R^{-\frac{1}{3}+\varepsilon}\|w\|_{\infty, \mathrm{B}^{R}}\right) .
$$

Similarly, we estimate

$$
\forall \varepsilon>0: \quad\left|I_{2}\right| \leq c_{7}\left(R^{-\frac{3}{2}}+R^{-\frac{1}{3}+\varepsilon}\|w\|_{\infty, \mathrm{B}^{R}}\right),
$$

and

$$
\forall \varepsilon>0: \quad\left|I_{3}\right| \leq c_{8}\left(R^{-\frac{3}{2}}+R^{-\frac{1}{3}+\varepsilon}\|v\|_{\infty, \mathrm{B}^{R}}\right) .
$$

Due to (5.22) and the fact that $\operatorname{supp} f$ is compact, we deduce

$$
\left|I_{4}\right| \leq c_{9} \int_{\operatorname{supp} f}\left(\frac{1}{\mathcal{T}} \int_{0}^{\mathcal{T}}\left|\Gamma^{\perp}(x-y, s)\right|^{2} \mathrm{~d} s\right)^{\frac{1}{2}} \mathrm{~d} y \leq c_{10} R^{-3}
$$

for $R$ sufficiently large. It follows from (5.43) and (5.45)-(5.48) that

$$
\forall \varepsilon>0: \quad|w(x, t)| \leq c_{11}\left(R^{-3}+R^{-\frac{3}{2}}+R^{-\frac{1}{3}+\varepsilon}\left(\|v\|_{\infty, \mathrm{B}^{R}}+\|w\|_{\infty, \mathrm{B}^{R}}\right)\right) .
$$




\section{Asymptotic structure}

We now turn our attention to $v$. Observe that $v$ satisfies

$$
\begin{cases}-\Delta v-\lambda \partial_{1} v=\mathcal{P}_{H} \mathcal{P} f-\mathcal{P}_{H}[\mathcal{P}[w \cdot \nabla w]+v \cdot \nabla v] & \text { in } \mathbb{R}^{3} \\ \operatorname{div} v=0 & \text { in } \mathbb{R}^{3}\end{cases}
$$

To establish a pointwise estimate of $v$, we follow essentially the proof of [18, Theorem IX.8.1]. By the summability properties (5.35) and (5.36) of the Oseen fundamental solution $\Gamma_{\mathrm{O}}^{\lambda}$, and the summability properties obtained from the assumptions on $v$ and $w$, it follows that convolution of $\Gamma_{\mathrm{O}}^{\lambda}$ with the right-hand side in (5.50) is well-defined in a classical sense. Recalling Lemma 3.6.2, we thus deduce that

$$
v=\Gamma_{\mathrm{O}}^{\lambda} *(\mathcal{P} f-\mathcal{P}[w \cdot \nabla w]-v \cdot \nabla v) .
$$

We again put $|x|=2 R$. Recalling (5.32) and that $f$ has compact support, we see for $R$ sufficiently large that

$$
\left|\Gamma_{\mathrm{O}}^{\lambda} * \mathcal{P} f(x)\right| \leq c_{12} \int_{\operatorname{supp} \mathcal{P} f} \frac{1}{|x-y|} \mathrm{d} y \leq c_{13} R^{-1} .
$$

Moreover, we can estimate

$$
\begin{aligned}
\left|\Gamma_{\mathrm{O}}^{\lambda} * \mathcal{P}[w \cdot \nabla w](x)\right| & =\left|\int_{\mathbb{R}^{3}} \Gamma_{\mathrm{O}}^{\lambda}(x-y) \frac{1}{\mathcal{T}} \int_{0}^{\mathcal{T}} w(y, t) \cdot \nabla w(y, t) \mathrm{d} t \mathrm{~d} y\right| \\
& \leq \frac{1}{\mathcal{T}} \int_{0}^{\mathcal{T}} \int_{\mathrm{B}_{R}} \frac{1}{R}|w \cdot \nabla w| \mathrm{d} y \mathrm{~d} t+\frac{1}{\mathcal{T}} \int_{0}^{\mathcal{T}} \int_{\mathrm{B}^{R}} \frac{|w(y, t)|}{|x-y|}|\nabla w(y, t)| \mathrm{d} y \mathrm{~d} t \\
& \leq c_{14} R^{-1}+\frac{1}{\mathcal{T}} \int_{0}^{\mathcal{T}}\left(\int_{\mathrm{B}^{R}} \frac{|w(y, t)|^{2}}{|x-y|^{2}} \mathrm{~d} y\right)^{\frac{1}{2}}\left(\int_{\mathrm{B}^{R}}|\nabla w(y, t)|^{2} \mathrm{~d} y\right)^{\frac{1}{2}} \mathrm{~d} t,
\end{aligned}
$$

where we use in the last estimate that $w \cdot \nabla w \in L^{1}(G)$. We can use the Hardy-type inequality from Lemma 2.3.3 to increase the remaining integral on the right-hand side above by

$$
\frac{1}{\mathcal{T}} \int_{0}^{\mathcal{T}}\left(\int_{\mathrm{B}^{R}} \frac{|w(y, t)|^{2}}{|x-y|^{2}} \mathrm{~d} y\right)^{\frac{1}{2}}\left(\int_{\mathrm{B}^{R}}|\nabla w(y, t)|^{2} \mathrm{~d} y\right)^{\frac{1}{2}} \mathrm{~d} t \leq c_{15} \frac{1}{\mathcal{T}} \int_{0}^{\mathcal{T}} \int_{\mathrm{B}^{R}}|\nabla w(y, t)|^{2} \mathrm{~d} y \mathrm{~d} t,
$$

whence by Corollary 5.1.2 we obtain

$$
\forall \varepsilon>0: \quad\left|\Gamma_{\mathrm{O}}^{\lambda} * \mathcal{P}[w \cdot \nabla w](x)\right| \leq c_{16} R^{-1+\varepsilon} .
$$

In a similar manner we show

$$
\forall \varepsilon>0: \quad\left|\Gamma_{\mathrm{O}}^{\lambda} * \mathcal{P}[v \cdot \nabla v](x)\right| \leq c_{17} R^{-1+\varepsilon} .
$$


We conclude that

$$
\forall \varepsilon>0: \quad|v(x)| \leq c_{18} R^{-1+\varepsilon} .
$$

Inserting (5.52) into (5.49), we then find

$$
\forall \varepsilon>0: \quad|w(x, t)| \leq c_{19}\left(R^{-\frac{1}{3}+\varepsilon}\|w\|_{\infty, \mathrm{B}^{R}}+R^{-\frac{4}{3}+\varepsilon}\right) .
$$

In particular

$$
\forall \varepsilon>0: \quad|w(x, t)| \leq c_{20} R^{-\frac{1}{3}+\varepsilon} .
$$

Inserting now (5.54) into (5.53), we deduce

$$
\forall \varepsilon>0: \quad|w(x, t)| \leq c_{21}\left(R^{-\frac{2}{3}+\varepsilon}\|w\|_{\infty, \mathrm{B}^{R}}+R^{-\frac{4}{3}+\varepsilon}\right) .
$$

Iterating this argument, we finally obtain

$$
\forall \varepsilon>0: \quad|w(x, t)| \leq c_{22}|x|^{-\frac{4}{3}+\varepsilon} .
$$

We now return to the representation formula (5.51) of $v$. Recalling the summability properties (5.37) and (5.38) of $\nabla \Gamma_{\mathrm{O}}^{\lambda}$, we estimate

$$
\begin{aligned}
\left|\Gamma_{\mathrm{O}}^{\lambda} * \mathcal{P}[w \cdot \nabla w](x)\right| & =\left|\frac{1}{\mathcal{T}} \int_{0}^{\mathcal{T}} \int_{\mathbb{R}^{3}} \Gamma_{\mathrm{O}}^{\lambda}(x-y) \operatorname{div}[w \otimes w](y, t) \mathrm{d} y \mathrm{~d} t\right| \\
& \leq c_{23} \frac{1}{\mathcal{T}} \int_{0} \int_{\mathbb{R}^{3}}\left|\nabla \Gamma_{\mathrm{O}}^{\lambda}(x-y)\right||w(y, t)|^{2} \mathrm{~d} y \mathrm{~d} t .
\end{aligned}
$$

We then use (5.33) to estimate

$$
\begin{aligned}
\frac{1}{\mathcal{T}} \int_{0}^{\mathcal{T}} \int_{\mathrm{B}_{R}}\left|\nabla \Gamma_{\mathrm{O}}^{\lambda}(x-y)\right||w(y, t)|^{2} \mathrm{~d} y \mathrm{~d} t & \leq c_{24} R^{-\frac{3}{2}} \frac{1}{\mathcal{T}} \int_{0}^{\mathcal{T}} \int_{\mathrm{B}_{R}}|w(y, t)|^{2} \mathrm{~d} y \mathrm{~d} t \\
& \leq c_{25} R^{-\frac{3}{2}}
\end{aligned}
$$

where we in the last inequality recall that $w \in L^{2}(G)$. Moreover, in view of (5.34) and 
(5.56), we see that

$$
\begin{aligned}
\frac{1}{\mathcal{T}} \int_{0}^{\mathcal{T}} \int_{\mathrm{B}_{3 R, R}}\left|\nabla \Gamma_{\mathrm{O}}^{\lambda}(x-y)\right||w(y, t)|^{2} \mathrm{~d} y \mathrm{~d} t & \leq c_{26} R^{-\frac{8}{3}+\varepsilon} \int_{\mathrm{B}_{3 R, R}}\left|\nabla \Gamma_{\mathrm{O}}^{\lambda}(x-y)\right| \mathrm{d} y \\
& \leq c_{26} R^{-\frac{8}{3}+\varepsilon} \int_{\mathrm{B}_{6 R}}\left|\nabla \Gamma_{\mathrm{O}}^{\lambda}(y)\right| \mathrm{d} y \\
& \leq c_{27} R^{-\frac{8}{3}+\varepsilon} \int_{0}^{6 R} \int_{\partial \mathrm{B}_{r}}\left|\nabla \Gamma_{\mathrm{O}}^{\lambda}(y)\right| \mathrm{d} S \mathrm{~d} r \\
& \leq c_{28} R^{-\frac{8}{3}+\varepsilon} \int_{0}^{6 R} r^{-\frac{1}{2}} \mathrm{~d} r \\
& \leq c_{29} R^{-\frac{8}{3}+\frac{1}{2}+\varepsilon}
\end{aligned}
$$

for all $\varepsilon>0$. Finally, employing again (5.33) and the fact that $w \in L^{2}(G)$, we estimate

$$
\frac{1}{\mathcal{T}} \int_{0}^{\mathcal{T}} \int_{\mathrm{B}^{3 R}}\left|\nabla \Gamma_{\mathrm{O}}^{\lambda}(x-y)\right||w(y, t)|^{2} \mathrm{~d} y \mathrm{~d} t \leq c_{30} R^{-\frac{3}{2}} .
$$

We thus conclude that

$$
\left|\Gamma_{\mathrm{O}}^{\lambda} * \mathcal{P}[w \cdot \nabla w](x)\right| \leq c_{31}|x|^{-\frac{3}{2}} .
$$

The other terms in the representation formula (5.51) for $v$ also appear in the analogous representation formula for a solution to the classical, steady-state Navier-Stokes system. We can therefore estimate them using well-known methods. More specifically, in view of (5.52) we can use the arguments from the proof of [18, Theorem IX.8.1] to obtain

$$
\forall \varepsilon>0: \quad\left|\Gamma_{\mathrm{O}}^{\lambda} *[v \cdot \nabla v](x)\right| \leq c_{32}|x|^{-\frac{3}{2}+\varepsilon}
$$

and

$$
\left|\Gamma_{\mathrm{O}}^{\lambda} * \mathcal{P} f(x)-\Gamma_{\mathrm{O}}^{\lambda}(x) \cdot\left(\int_{\mathbb{R}^{3}} \mathcal{P} f\right)\right| \leq c_{33}|x|^{-\frac{3}{2}} .
$$

We therefore finally deduce, recalling (5.51) and (5.56), that

$$
\begin{aligned}
u(x, t) & =v(x)+w(x, t) \\
& =\Gamma_{\mathrm{O}}^{\lambda}(x) \cdot\left(\int_{\mathbb{R}^{3}} \mathcal{P} f\right)+\mathscr{R}(x, t)
\end{aligned}
$$

with $\mathscr{R}(x, t)$ satisfying (5.41). 
Remark 5.1.7. The conditions on the solution in Theorem 5.1.6 are clearly satisfied by the solution established in Theorem 4.1.5. We have thus obtained both existence and an asymptotic expansion of a strong solution to the time-periodic Navier-Stokes problem in the case $\lambda \neq 0$. Observe that, in contrast to Theorem 4.1.5, no smallness condition is needed on the data in Theorem 5.1.6. Further observe, by going through the proof of Theorem 5.1.6, that the assumptions in Theorem 5.1.6 could be weakened. The condition (5.39) has been chosen only to keep a connection with the class of strong solutions from Theorem 4.1.5.

Remark 5.1.8. It is well-known, see for example [17, Chapter VII.3], that the Oseen fundamental solution $\Gamma_{\mathrm{O}}^{\lambda}$ has order of decay $|x|^{-1+\sigma}$ in a parabolic so-called wake region in the direction $\mathrm{e}_{1}$, where $\sigma$ depends on the angle of the specific parabolic region under consideration. More precisely, $\sigma$ is zero if the angle is zero, and increases as the angle increases. From the asymptotic expansion established in (5.40) one can therefore conclude the existence of such a wake region also for a strong solution to the time-periodic Navier-Stokes system in the case $\lambda \neq 0$. Note that the size of the manifested wake region is determined by the decay rate obtained for the remainder term $\mathscr{R}(x, t)$. The rate $|x|^{-4 / 3+\varepsilon}$ shown in Theorem 5.1.6 is probably not optimal. In the steady-state case, which as we recall is a special case of the time-periodic problem, the uniform decay rate $|x|^{-3 / 2+\varepsilon}$ can be shown; see for example [18, Theorem IX.8.1]. Going through the proof of Theorem 5.1.6, we see that the decay rate of $\mathscr{R}(x, t)$ is determined by estimate $(5.22)$ of the fundamental solution $\Gamma^{\perp}$. We observe that an improvement in estimate (5.22) immediately implies a similar improvement in the decay of $\mathscr{R}(x, t)$, but leave it as an open question if this is actually possible to obtain.

\subsection{Energy}

An asymptotic expansion of a solution to (5.1) can be used to study the physical properties of the flow. One such property is the kinetic energy. For a steady-state flow it is known in the case $\lambda \neq 0$ that the kinetic energy is infinite unless the (time-independent) data $f$ satisfies

$$
\int_{\mathbb{R}^{3}} f(x) \mathrm{d} x=0 .
$$

In this section, we shall generalize this result to the time-periodic Navier-Stokes problem. More precisely, we shall show in the case $\lambda \neq 0$ that the kinetic energy of a strong solution to (5.1) is infinite unless

$$
\frac{1}{\mathcal{T}} \int_{0}^{\mathcal{T}} \int_{\mathbb{R}^{3}} f(x, t) \mathrm{d} x \mathrm{~d} t=0
$$

is satisfied for the (time-periodic) data $f$. 


\section{Asymptotic structure}

Although the asymptotic expansion (5.40) established in Theorem 5.1.6 indicates the aforementioned property of the kinetic energy, it is not possible to derive the property directly from (5.40). This is due to the pointwise estimate of the remainder term $\mathscr{R}(x, t)$, which does not reveal any information on the $L^{2}\left(\mathbb{R}^{3}\right)$-norm, that is, the kinetic energy of $\mathscr{R}(x, t)$. Below, we shall therefore investigate more carefully the summability of $\mathscr{R}(x, t)$. We start with a lemma.

Lemma 5.2.1. If

$$
\forall q \in(1, \infty): \quad H \in W^{1, q}\left(\mathbb{R}^{3}\right)^{3 \times 3}
$$

then

$$
\forall q \in(4 / 3, \infty): \quad \Gamma_{\mathrm{O}}^{\lambda} * \operatorname{div} H \in L^{q}\left(\mathbb{R}^{3}\right)^{3} .
$$

Proof. By (5.35), (5.36), and Young's inequality, the convolution $\Gamma_{\mathrm{O}}^{\lambda} * \operatorname{div} H$ is welldefined. Taking into account (5.37) and (5.38), we further see that

$$
\Gamma_{\mathrm{O}}^{\lambda} * \operatorname{div} H=\nabla \Gamma_{\mathrm{O}}^{\lambda} * H \in L^{q}\left(\mathbb{R}^{3}\right)^{3}
$$

for all $q \in(4 / 3, \infty)$.

We shall now determine the summability of the term $\mathscr{R}(x, t)$ in $(5.40)$. The result is important in its own right, so we state it as a theorem.

Theorem 5.2.2. Let $\lambda>0, f \in C_{0}^{\infty}(G)^{3}$, and $(u, \mathfrak{p})$ be a solution to (5.4) with $u=v+w$ satisfying for some $q \in\left(1, \frac{4}{3}\right)$ and $r \in(4, \infty)$ :

$$
(v, w, \mathfrak{p}) \in \mathrm{X}_{\sigma, \text { Oseen }}^{q, r}\left(\mathbb{R}^{3}\right) \times W_{\sigma, \perp}^{1,2, q, r}(G) \times \mathrm{X}_{\mathrm{P}}^{q, r}(G) .
$$

Then

$$
u(x, t)=\Gamma_{\mathrm{O}}^{\lambda}(x) \cdot\left(\frac{1}{\mathcal{T}} \int_{0}^{\mathcal{T}} \int_{\mathbb{R}^{3}} f \mathrm{~d} x \mathrm{~d} t\right)+\mathscr{R}(x, t)
$$

with

$$
\begin{array}{ll}
\forall s \in(4 / 3, \infty): & \mathscr{R} \in L^{\infty}\left((0, \mathcal{T}) ; L^{s}\left(\mathbb{R}^{3} \backslash \mathrm{B}_{1}\right)^{3}\right), \\
\forall s \in(4 / 3,3): & \mathscr{R} \in L^{\infty}\left((0, \mathcal{T}) ; L^{s}\left(\mathbb{R}^{3}\right)^{3}\right) .
\end{array}
$$

Proof. Observe first, since $w$ belongs to $W^{1,2, q}(G)$, that classical Sobolev embedding implies $w \in L^{\infty}\left((0, \mathcal{T}) ; L^{q}\left(\mathbb{R}^{3}\right)^{3}\right)$. From Lemma 4.1 .2 we already know that $w \in$ $L^{\infty}\left((0, \mathcal{T}) ; L^{\infty}\left(\mathbb{R}^{3}\right)^{3}\right)$. It follows that

$$
\forall s \in(q, \infty): \quad w \in L^{\infty}\left((0, \mathcal{T}) ; L^{s}\left(\mathbb{R}^{3}\right)^{3}\right) .
$$


Consequently, (5.60) can be established by expanding $v$ appropriately. As in the proof of Theorem 5.1.6, we find that

$$
v=\Gamma_{\mathrm{O}}^{\lambda} *(\mathcal{P} f-\mathcal{P}[w \cdot \nabla w]-v \cdot \nabla v) .
$$

Recall that $\mathcal{P}[w \cdot \nabla w]=\operatorname{div} \mathcal{P}[w \otimes w]$ and $v \cdot \nabla v=\operatorname{div} v \otimes v$. From the summability of $w$ and $v$ ensured by assumption (5.59), it therefore follows that both $\mathcal{P}[w \cdot \nabla w]$ and $v \cdot \nabla v$ have the form $\operatorname{div} H$ with $H$ satisfying (5.57). Consequently

$$
\forall s \in\left(\frac{4}{3}, \infty\right): \quad \Gamma_{\mathrm{O}}^{\lambda} *(\mathcal{P}[w \cdot \nabla w]+v \cdot \nabla v) \in L^{s}\left(\mathbb{R}^{3}\right)^{3} .
$$

Finally, it is well known, see for example [17, Chapter VII.3, p.373], that

$$
\Gamma_{\mathrm{O}}^{\lambda} * \mathcal{P} f(x)=\Gamma_{\mathrm{O}}^{\lambda}(x) \cdot\left(\int_{\mathbb{R}^{3}} \mathcal{P} f(y) \mathrm{d} y\right)+O\left(\nabla \Gamma_{\mathrm{O}}^{\lambda}(x)\right) \quad \text { as }|x| \rightarrow \infty,
$$

from which we infer, by the summability property $(5.37)$ of $\nabla \Gamma_{\mathrm{O}}^{\lambda}$, that

$$
\forall s \in\left(\frac{4}{3}, \infty\right): \Gamma_{\mathrm{O}}^{\lambda} * \mathcal{P} f-\Gamma_{\mathrm{O}}^{\lambda} \cdot\left(\int_{\mathbb{R}^{3}} \mathcal{P} f(y) \mathrm{d} y\right) \in L^{s}\left(\mathbb{R}^{3} \backslash \mathrm{B}_{1}\right)^{3} .
$$

Hence, the identity

$$
\begin{aligned}
u=v+w=\Gamma_{\mathrm{O}}^{\lambda} \cdot\left(\int_{\mathbb{R}^{3}} \mathcal{P} f(y) \mathrm{d} y\right) & +\Gamma_{\mathrm{O}}^{\lambda} * \mathcal{P} f-\Gamma_{\mathrm{O}}^{\lambda} \cdot\left(\int_{\mathbb{R}^{3}} \mathcal{P} f(y) \mathrm{d} y\right) \\
& -\Gamma_{\mathrm{O}}^{\lambda} *(\mathcal{P}[w \cdot \nabla w]+v \cdot \nabla v)+w
\end{aligned}
$$

together with (5.63), (5.64), (5.65), and the fact that

$$
\int_{\mathbb{R}^{3}} \mathcal{P} f(y) \mathrm{d} y=\frac{1}{\mathcal{T}} \int_{0}^{\mathcal{T}} \int_{\mathbb{R}^{3}} f \mathrm{~d} x \mathrm{~d} t
$$

enables us to conclude

$$
u(x, t)=\Gamma_{\mathrm{O}}^{\lambda}(x) \cdot\left(\frac{1}{\mathcal{T}} \int_{0}^{\mathcal{T}} \int_{\mathbb{R}^{3}} f \mathrm{~d} x \mathrm{~d} t\right)+\mathscr{R}(x, t)
$$

with $\mathscr{R}$ satisfying (5.61). Due to the local summability (5.36) of $\Gamma_{\mathrm{O}}^{\lambda}$ and the summability (4.11) and (5.63) of $v$ and $w$, respectively, we further deduce (5.62).

Remark 5.2.3. The asymptotic expansion (5.60) can be obtained under assumptions on the solution that are weaker than (5.59). The stronger condition (5.59) is chosen here only because the solutions established in Theorem 4.1.5 clearly belong to this class. 


\section{Asymptotic structure}

In view of (6.9) in the following chapter, it is even likely that the weak solution from Theorem 6.3.1 satisfies an expansion of type

$$
u(x, t)=\Gamma_{\mathrm{O}}^{\lambda}(x) \cdot\left(\frac{1}{\mathcal{T}} \int_{0}^{\mathcal{T}} \int_{\mathbb{R}^{3}} f \mathrm{~d} x \mathrm{~d} t\right)+\mathscr{R}(x, t)
$$

with $\mathscr{R} \in L^{\infty}\left((0, \mathcal{T}) ; L^{2}\left(\mathbb{R}^{3}\right)^{3}\right)$. This conjecture will be the source of investigation in a forthcoming paper. We leave it at this point as an open question.

Finally, we can conclude the following property of the kinetic energy of the fluid flow corresponding to the strong solution found in Theorem 4.1.5.

Theorem 5.2.4. Let $q \in\left(1, \frac{4}{3}\right), r \in(4, \infty), f \in C_{0}^{\infty}(G)^{3}$, and $(u, \mathfrak{p})$ be the solution from Theorem 4.1.5. The kinetic energy of $u$ is finite, that is,

$$
\|u\|_{L^{\infty}\left((0, \mathcal{T}) ; L^{2}\left(\mathbb{R}^{3}\right)\right)}<\infty,
$$

if and only if

$$
\frac{1}{\mathcal{T}} \int_{0}^{\mathcal{T}} \int_{\mathbb{R}^{3}} f(x, t) \mathrm{d} x \mathrm{~d} t=0 .
$$

Proof. Recall, see for example [17, Chapter VII.3, (3.30)], that no entries of the tensor $\Gamma_{\mathrm{O}}^{\lambda}$ belong to $L^{2}\left(\mathbb{R}^{3}\right)$. More precisely, $\left\|\left[\Gamma_{\mathrm{O}}^{\lambda}\right]_{j k}\right\|_{2}=\infty$ for all $j, k=1,2,3$. The theorem therefore follows directly from (5.60) and (5.62).

\subsection{Flow past a rotating body}

We can apply Theorem 5.1.6 to establish an asymptotic expansion at spatial infinity of a Navier-Stokes flow past a rotating body that is steady in a frame attached to the body. The equations of motion for a Navier-Stokes liquid flowing past a rotating body, written in a frame attached to the body, are given by (3.87). Thus, a flow that is steady, i.e. time independent, with respect to this frame is governed by the system

$$
\begin{cases}-\Delta v+\nabla p+v \cdot \nabla v-\lambda \partial_{1} v-\omega\left(\mathrm{e}_{1} \wedge x \cdot \nabla v-\mathrm{e}_{1} \wedge v\right)=0 & \text { in } \Omega, \\ \operatorname{div} v=0 & \text { in } \Omega, \\ v=\lambda \mathrm{e}_{1}+\left(\omega \mathrm{e}_{1}\right) \wedge x & \text { on } \partial \Omega, \\ \lim _{|x| \rightarrow \infty} v(x)=0, & \end{cases}
$$

where $v: \Omega \rightarrow \mathbb{R}^{3}$ and $p: \Omega \rightarrow \mathbb{R}$ denote the Eulerian velocity and pressure of the liquid, respectively. Recall that $\Omega \subset \mathbb{R}^{3}$ is an exterior domain with $\mathbb{R}^{3} \backslash \bar{\Omega}$ being the domain occupied by the body. Moreover, $\omega \mathrm{e}_{1} \in \mathbb{R}^{3}$ is the angular and $\lambda \mathrm{e}_{1} \in \mathbb{R}^{3}$ the translational velocity of the body, which we take to be directed along the same axis. This assumption 
can be made without loss of generality, provided the angular and translational velocity are not orthogonal. In the general case, one can apply a simple transformation to obtain (5.67); see for example [26]. For simplicity, we have assumed that no external forces act on the liquid. In this case, the only driving force of the flow is the motion of the body.

In the case both angular and translational velocity of the body are nonzero, that is, the body both translates and rotates, we can employ Theorem 5.1.6 to identify the asymptotic profile as $|x| \rightarrow \infty$ of the velocity field $v(x)$ in terms of the Oseen fundamental solution $\Gamma_{\mathrm{O}}^{\lambda}$ introduced in (5.30).

Theorem 5.3.1. Let $\Omega \subset \mathbb{R}^{3}$ be a $C^{2}$-smooth exterior domain, and $\lambda, \omega>0$. A solution

$$
(v, p) \in D^{1,2}(\Omega)^{3} \cap L^{6}(\Omega)^{3} \cap W_{l o c}^{2,2}(\bar{\Omega})^{3} \times W_{l o c}^{1,2}(\bar{\Omega})
$$

to (5.67) satisfies the asymptotic expansion

$$
v(x)=\Gamma_{\mathrm{O}}^{\lambda}(x) \cdot\left(\mathcal{F} \cdot \mathrm{e}_{1}\right) \mathrm{e}_{1}+\mathscr{R}(x),
$$

with

$$
\forall \varepsilon>0:|\mathscr{R}(x)| \leq C_{34}|x|^{-\frac{4}{3}+\varepsilon} \quad \text { for }|x| \geq 1
$$

and

$$
\mathcal{F}:=\int_{\partial \Omega} \mathrm{T}(v, p) \cdot n \mathrm{~d} S .
$$

Here, $\mathrm{T}(v, p):=\nabla v+\nabla v^{\top}-p I$ denotes the Cauchy stress tensor of the liquid.

Proof. Asymptotic properties of a solution $(v, p)$ to $(5.67)$ in the class $(5.68)$ have been studied extensively in [23]. In [23, Theorem 4.4] it is shown for sufficiently large $\rho>0$ that

$$
\forall q \in(1,2): v \in \mathrm{X}_{\sigma, \text { Oseen }}^{q}\left(\mathrm{~B}^{\rho}\right) .
$$

Moreover, in [23, Theorem 5.1 and Theorem 5.2] pointwise decay estimates of $v$ and $\nabla v$ are established. From these estimates it follows that

$$
v \in L^{\infty}\left(\mathrm{B}^{\rho}\right)^{3} \quad \text { and } \quad \nabla v \in L^{\infty}\left(\mathrm{B}^{\rho}\right)^{3 \times 3} .
$$

In addition, putting the lower order terms $-\lambda \partial_{1} v-\omega\left(\mathrm{e}_{1} \wedge x \cdot \nabla v-\mathrm{e}_{1} \wedge v\right)$ in the first equation in (5.67) on the right-hand side, we obtain by a boot-strapping argument utilizing standard regularity theory for the classical Navier-Stokes system, see for example [17, Theorem VIII.5.1], that

$$
(v, p) \in C^{\infty}\left(\mathrm{B}^{\rho}\right)^{3} \times C^{\infty}\left(\mathrm{B}^{\rho}\right) .
$$




\section{Asymptotic structure}

We shall transform $(v, p)$ into a solution to a whole-space problem. For this purpose, choose $\rho>0$ so large that (5.72)-(5.74) are satisfied and $\mathbb{R}^{3} \backslash \Omega \subset \mathrm{B}_{\rho}$. Let $\chi_{\rho} \in C^{\infty}\left(\mathbb{R}^{3}\right)$ be a "cut-off" function with $\chi_{\rho}=0$ in $\mathrm{B}_{\rho}$ and $\chi_{\rho}=1$ in $\mathbb{R}^{3} \backslash \mathrm{B}_{2 \rho}$. We then define

$$
\begin{aligned}
& w: \mathbb{R}^{3} \rightarrow \mathbb{R}^{3}, \quad w(x):=\chi_{\rho}(x) v(x)-\mathfrak{B}\left[\nabla \chi_{\rho} \cdot v\right](x), \\
& \pi: \mathbb{R}^{3} \rightarrow \mathbb{R}, \quad \pi(x):=\chi_{\rho}(x) p(x),
\end{aligned}
$$

where $\mathfrak{B}$ denotes the so-called "Bogovskil operator", that is, an operator

$$
\mathfrak{B}: C_{0}^{\infty}\left(\mathrm{B}_{2 \rho}\right) \rightarrow C_{0}^{\infty}\left(\mathrm{B}_{2 \rho}\right)^{3}
$$

with the property that $\operatorname{div} \mathfrak{B}(f)=f$ whenever $\int_{\mathrm{B}_{2 \rho}} f(x) \mathrm{d} x=0$. We refer to $[17$, Theorem III.3.2] for details on this operator. Observe that

$$
\begin{aligned}
\int_{\mathrm{B}_{2 \rho}} \nabla \chi_{\rho} \cdot v \mathrm{~d} x & =\int_{\mathrm{B}_{2 \rho}} \operatorname{div}\left(\chi_{\rho} v\right) \mathrm{d} x \\
& =\int_{\partial \mathrm{B}_{2 \rho}} v \cdot n \mathrm{~d} x \\
& =\int_{\partial \Omega} v \cdot n \mathrm{~d} S \\
& =\int_{\partial \Omega}\left(\lambda \mathrm{e}_{1}+\left(\omega \mathrm{e}_{1}\right) \wedge x\right) \cdot n \mathrm{~d} S=\int_{\mathbb{R}^{3} \backslash \bar{\Omega}} \operatorname{div}\left(\lambda \mathrm{e}_{1}+\left(\omega \mathrm{e}_{1}\right) \wedge x\right) \mathrm{d} x=0 .
\end{aligned}
$$

It follows that $(w, \pi)$ is a smooth solution to the whole-space problem

$$
\begin{cases}-\Delta w+\nabla \pi-\lambda \partial_{1} w-\omega\left(\mathrm{e}_{1} \wedge x \cdot \nabla w-\mathrm{e}_{1} \wedge w\right)=h-w \cdot \nabla w & \text { in } \mathbb{R}^{3}, \\ \operatorname{div} w=0 & \text { in } \mathbb{R}^{3},\end{cases}
$$

where $h$ is a function with

$$
h \in C_{0}^{\infty}\left(\mathbb{R}^{3}\right)^{3}, \quad \operatorname{supp} h \subset \mathrm{B}_{2 \rho} .
$$

Due to (5.72)-(5.74), we clearly have

$$
\forall q \in(1,2): w \in \mathrm{X}_{\sigma, \text { Oseen }}^{q}\left(\mathbb{R}^{3}\right), \quad w \in L^{\infty}\left(\mathbb{R}^{3}\right)^{3}, \quad \text { and } \quad \nabla w \in L^{\infty}\left(\mathbb{R}^{3}\right)^{3 \times 3} .
$$

From these properties it is easy to verify for any $r \in(8, \infty)$ that

$$
w \cdot \nabla w \in L^{\frac{6}{5}}\left(\mathbb{R}^{3}\right)^{3} \cap L^{r}\left(\mathbb{R}^{3}\right)^{3} .
$$

In the next step, we transform $(w, \pi)$ into a solution to a time-periodic problem. For this purpose, we recall the definition of the $\frac{2 \pi}{\omega}$-periodic rotation matrix $Q(t)$ in (3.96), put $\mathcal{T}:=\frac{2 \pi}{\omega}$, and define

$$
\begin{aligned}
& u: G \rightarrow \mathbb{R}^{3}, \quad u(x, t):=Q(t) w\left(Q(t)^{\top} x\right), \\
& \mathfrak{p}: G \rightarrow \mathbb{R}, \quad \mathfrak{p}(x, t):=\pi\left(Q(t)^{\top} x\right), \\
& H: G \rightarrow \mathbb{R}^{3}, \quad H(x, t):=Q(t) h\left(Q(t)^{\top} x\right),
\end{aligned}
$$


where we as usual put $G:=\mathbb{R} / \mathcal{T} \mathbb{Z}$. Then $(u, \mathfrak{p})$ is a smooth solution to

$$
\begin{cases}\partial_{t} u-\Delta u-\lambda \partial_{1} u+\nabla \mathfrak{p}=H-u \cdot \nabla u & \text { in } G, \\ \operatorname{div} u=0 & \text { in } G .\end{cases}
$$

Moreover, (5.78) and (5.79) yield

$$
H+u \cdot \nabla u \in L^{\frac{6}{5}}(G)^{3} \cap L^{r}(G)^{3}
$$

for any $r \in(8, \infty)$. Utilizing Theorem 4.1 .3 in combination with the uniqueness properties from Lemma 3.6.2 and Lemma 3.6.4, we obtain

$$
\left(\mathcal{P} u, \mathcal{P}_{\perp} u\right)=A_{\lambda, \sigma}^{-1} \mathcal{P}_{H}(H+u \cdot \nabla u) \in \mathrm{X}_{\sigma, \text { Oseen }}^{\frac{6}{5}, r}\left(\mathbb{R}^{3}\right) \times W_{\sigma, \perp}^{1,2, \frac{6}{5}, r}(G) .
$$

In view of Lemma 4.1.4, we let $\tilde{\mathfrak{p}}=\operatorname{grad}^{-1}\left[\mathrm{Id}-\mathcal{P}_{H}\right](H+u \cdot \nabla u) \in \mathrm{X}_{\mathrm{P}}^{\frac{6}{5}}, r(G)$ and observe that $(u, \tilde{\mathfrak{p}})$ also solves $(5.80)$, although $\tilde{\mathfrak{p}}$ may differ from $\mathfrak{p}$. Finally, we are now in a position where we can apply Theorem 5.1.6 to $(u, \tilde{\mathfrak{p}})$ and obtain

$$
u(x, t)=\Gamma_{\mathrm{O}}^{\lambda}(x) \cdot\left(\frac{1}{\mathcal{T}} \int_{0}^{\mathcal{T}} \int_{\mathbb{R}^{3}} H(y, t) \mathrm{d} y \mathrm{~d} t\right)+\mathscr{R}(x, t),
$$

with $\mathscr{R}(x, t)$ satisfying (5.41). In order to compute the integral in (5.81), we isolate $h$ in (5.77) and integrate the resulting identity. Recalling (5.78), we thus deduce

$$
\begin{array}{rl}
\int_{\mathbb{R}^{3}} & h \mathrm{~d} x \\
= & \int_{\mathrm{B}_{2 \rho}} \operatorname{div}\left[-\mathrm{T}(w, \pi)-\lambda w \otimes \mathrm{e}_{1}-\omega w \otimes\left(\mathrm{e}_{1} \wedge x\right)+\omega\left(\mathrm{e}_{1} \wedge x\right) \otimes w+w \otimes w\right] \mathrm{d} x \\
= & \int_{\partial \mathrm{B}_{2 \rho}}\left[-\mathrm{T}(w, \pi)-\lambda w \otimes \mathrm{e}_{1}-\omega w \otimes\left(\mathrm{e}_{1} \wedge x\right)+\omega\left(\mathrm{e}_{1} \wedge x\right) \otimes w+w \otimes w\right] \cdot n \mathrm{~d} S \\
= & \int_{\partial \mathrm{B}_{2 \rho}}\left[-\mathrm{T}(v, p)-\lambda v \otimes \mathrm{e}_{1}-\omega v \otimes\left(\mathrm{e}_{1} \wedge x\right)+\omega\left(\mathrm{e}_{1} \wedge x\right) \otimes v+v \otimes v\right] \cdot n \mathrm{~d} S \\
= & -\int_{\partial \Omega}\left[-\mathrm{T}(v, p)-\lambda v \otimes \mathrm{e}_{1}-\omega v \otimes\left(\mathrm{e}_{1} \wedge x\right)+\omega\left(\mathrm{e}_{1} \wedge x\right) \otimes v+v \otimes v\right] \cdot n \mathrm{~d} S \\
& +\int_{\Omega \cap \mathrm{B}_{2 \rho}} \operatorname{div}\left[-\mathrm{T}(v, p)-\lambda v \otimes \mathrm{e}_{1}-\omega v \otimes\left(\mathrm{e}_{1} \wedge x\right)+\omega\left(\mathrm{e}_{1} \wedge x\right) \otimes v+v \otimes v\right] \mathrm{d} x \\
= & -\int_{\partial \Omega}\left[-\mathrm{T}(v, p)-\lambda v \otimes \mathrm{e}_{1}-\omega v \otimes\left(\mathrm{e}_{1} \wedge x\right)+\omega\left(\mathrm{e}_{1} \wedge x\right) \otimes v+v \otimes v\right] \cdot n \mathrm{~d} S,
\end{array}
$$




\section{Asymptotic structure}

where the last equality is due to the fact that $(v, p)$ solves (5.67). Inserting the boundary values from (5.67) for $v$ on $\partial \Omega$, we verify by elementary calculations similar to (5.76) that all but the first term in the last integral above vanish. Thus

$$
\int_{\mathbb{R}^{3}} h \mathrm{~d} x=\int_{\partial \Omega} \mathrm{T}(v, p) \cdot n \mathrm{~d} S=\mathcal{F} .
$$

We can now deduce

$$
\begin{aligned}
\frac{1}{\mathcal{T}} \int_{0}^{\mathcal{T}} \int_{\mathbb{R}^{3}} H(y, t) \mathrm{d} y \mathrm{~d} t & =\frac{1}{\mathcal{T}} \int_{0}^{\mathcal{T}} \int_{\mathbb{R}^{3}} Q(t) h\left(Q(t)^{\top} y\right) \mathrm{d} y \mathrm{~d} t \\
& =\frac{1}{\mathcal{T}} \int_{0}^{\mathcal{T}} Q(t)\left(\int_{\mathbb{R}^{3}} h(y) \mathrm{d} y\right) \mathrm{d} t \\
& =\frac{1}{\mathcal{T}} \int_{0}^{\mathcal{T}} Q(t) \mathcal{F} \mathrm{d} t=\left(\mathcal{F} \cdot \mathrm{e}_{1}\right) \mathrm{e}_{1} .
\end{aligned}
$$

We then return to (5.81) and find that

$$
w(x)=Q^{\top}(t) \Gamma_{\mathrm{O}}^{\lambda}(Q(t) x) \cdot\left(\mathcal{F} \cdot \mathrm{e}_{1}\right) \mathrm{e}_{1}+Q^{\top}(t) \mathscr{R}(Q(t) x, t) .
$$

The first column of the Oseen Fundamental solution, i.e., the vector field $\Gamma_{\mathrm{O}}^{\lambda} \cdot \mathrm{e}_{1}$, is invariant with respect to rotation around the axis $\mathrm{e}_{1}$; a property one may verify directly or simply from the fact that the system (5.31) is invariant with respect to such a rotation. Consequently,

$$
w(x)=\Gamma_{\mathrm{O}}^{\lambda}(x) \cdot\left(\mathcal{F} \cdot \mathrm{e}_{1}\right) \mathrm{e}_{1}+Q^{\top}(t) \mathscr{R}(Q(t) x, t) .
$$

Incidentally, the term $Q^{\top}(t) \mathscr{R}(Q(t) x, t)$ is $t$-independent. As a consequence of $\mathscr{R}$ satisfying (5.41), we find for any $\varepsilon>0$ and $|x| \geq 1$ that

$$
\left|Q^{\top}(t) \mathscr{R}(Q(t) x, t)\right|=|\mathscr{R}(Q(t) x, t)| \leq C_{33}|Q(t) x|^{-\frac{4}{3}+\varepsilon}=C_{33}|x|^{-\frac{4}{3}+\varepsilon} .
$$

Since $v(x)=w(x)$ for $|x| \geq 2 \rho$, the asymptotic expansion (5.69) therefore follows from (5.82).

Remark 5.3.2. Note that $\mathcal{F}$ in (5.71) equals the total force exerted by the liquid on the body. Since we in (5.67) consider the no-slip boundary condition, there is no contribution to the total force from momentum flux via the liquid-structure boundary.

Remark 5.3.3. The class (5.68) is the natural class of weak solutions for (5.67). In fact, existence of such a weak solution can be shown without any restrictions on the "size" of the data; see for example [21, Chapter XI]. ${ }^{2}$

\footnotetext{
${ }^{2}$ Chapter XI is new in the latest edition of the monograph [21].
} 
Remark 5.3.4. The asymptotic expansion (5.69) has already been shown in [39]. In [39], however, the decay of the remainder term $\mathscr{R}(x)$ as $|x| \rightarrow \infty$ is only established in terms of summability. More precisely, (5.69) is established in [39, Theorem 1.1] with

$$
\forall q \in(4 / 3, \infty): \mathscr{R} \in L^{q}(\Omega)^{3} .
$$

Since $\Gamma_{\mathrm{O}}^{\lambda} \cdot \mathrm{e}_{1} \notin L^{q}(\Omega)$ for any $q \in[4 / 3,2]$, it follows from (5.83) that $\mathscr{R}$ in a sense has a faster decay as $|x| \rightarrow \infty$ than the leading term in (5.69). This manifestation of $\mathscr{R}$ 's decay property does not reveal any information on the pointwise behavior of $\mathscr{R}$ as $|x| \rightarrow \infty$, though. In this respect, Theorem 5.3.1 constitutes an improvement. Observe that (5.83) actually follows by the same proof as above if we in (5.81) instead of Theorem 5.1.6 employ Theorem 5.2.2.

Remark 5.3.5. An asymptotic expansion of a solution to (5.67) has been established in the case $\lambda=0, \omega>0$ by FARWIG and HishidA in [8]. In this case, the leading term is not the Oseen fundamental solution, but the so-called Landau solution that was already mentioned in Section 1.6. In [8], the expansion is shown with a remainder term decaying faster than the leading term only in the sense of summability. In [7], a pointwise estimate of the remainder term was established by FARWIG, GALDI, and KYED. 



\section{Weak solutions}

In this chapter, an investigation of weak solutions to the time-periodic Navier-Stokes system

$$
\begin{cases}\partial_{t} u-\Delta u-\lambda \partial_{1} u+\nabla \mathfrak{p}+u \cdot \nabla u=f & \text { in } \mathbb{R}^{3} \times \mathbb{R}, \\ \operatorname{div} u=0 & \text { in } \mathbb{R}^{3} \times \mathbb{R}, \\ \lim _{|x| \rightarrow \infty} u(x, t)=0, & \\ u(\cdot, t+\mathcal{T})=u(\cdot, t) & \end{cases}
$$

is carried out. For a time-periodic right-hand side $f$ the existence of a weak time-periodic solution $u$ is shown. Moreover, by decomposing $u$ into a steady-state and time-periodic part, $u=u_{s}+u_{p}$, additional regularity properties will be established by studying $u_{s}$ and $u_{p}$ separately. In particular, we shall show that $u_{p} \in L^{\infty}\left(\mathbb{R} ; L^{2}\left(\mathbb{R}^{3}\right)^{3}\right)$, which is a fundamental new result. We emphasize that the result presented in this chapter requires no restriction on either the "size" or the structure of the data $f$.

The chapter is organized as follows: We first introduce in Section 6.1 a weak formulation of (6.1) on the torus group, including a definition of a weak solution. In Section 6.2 we briefly define the projection operator used to decompose functions into a steady and time-periodic part. In Section 6.3 we then show existence of at least one weak solution. To conclude that this solution is time periodic in a classical pointwise fashion, we establish in Section 6.4 continuity of the solution in an appropriate topology.

\subsection{Weak formulation on the torus group}

As mentioned in the introduction, we will use a functional analytic setting based solely on time-periodic functions. To accomplish this, we employ function spaces in which the time interval is replaced with the torus group $\mathbb{T}:=\mathbb{R} / \mathcal{T} \mathbb{Z}$, where $\mathcal{T}$ denotes the period of the data.

In a weak formulation, it turns out convenient to formulate the time-periodic problem in terms of the torus group. If the right-hand side $f: \mathbb{R} \rightarrow D_{0}^{-1,2}\left(\mathbb{R}^{3}\right)^{3}$ is $\mathcal{T}$ periodic, the appropriate function space for the data in a classical weak formulation will be $L^{2}\left(0, \mathcal{T} ; D_{0}^{-1,2}\left(\mathbb{R}^{3}\right)^{3}\right)$. The corresponding function space for the weak solution $u$ is then $L^{2}\left(0, \mathcal{T} ; D_{0}^{1,2}\left(\mathbb{R}^{3}\right)^{3}\right)$. However, since the elements of $L^{2}\left(0, \mathcal{T} ; D_{0}^{1,2}\left(\mathbb{R}^{3}\right)^{3}\right)$ do not have a pointwise definition, it does not make sense to require $u(0)=u(\mathcal{T})$. In other words, care must be taken in the weak formulation to obtain a setting in which the time-periodicity of the solution can be appropriately expressed. One way to achieve this is to work with the torus group $\mathbb{T}:=\mathbb{R} / \mathcal{T} \mathbb{Z}$ instead of the time interval $[0, \mathcal{T}]$. Assume 


\section{Weak solutions}

namely we can find a weak solution $u \in L^{2}\left(\mathbb{T} ; D_{0}^{1,2}\left(\mathbb{R}^{3}\right)^{3}\right)$, then proper $\mathcal{T}$-periodicity of $u$ can simply be established by showing additional time regularity of $u$. More specifically, one needs $u \in C(\mathbb{T} ; X)$ for some topological space $X$ to conclude $u(0)=u(\mathcal{T})$.

\subsubsection{Function spaces}

Throughout the chapter we let $\mathbb{T}$ denote the torus group $\mathbb{R} / \mathcal{T} \mathbb{Z}$ endowed with the canonical quotient topology. In addition, $\mathbb{T}$ is equipped with the normalized Haarmeasure, which is equivalent to the normalized Lebesgue measure on $[0, \mathcal{T})$. More precisely,

$$
\forall \varphi \in C_{0}(\mathbb{T}): \quad \int_{\mathbb{T}} \varphi \mathrm{d} t:=\frac{1}{\mathcal{T}} \int_{0}^{\mathcal{T}} \varphi(t) \mathrm{d} t .
$$

For simplicity, we use $\mathrm{d} t$ to denote both the normalized Haar-measure on $G$ and the Lebesgue measure on $[0, \mathcal{T})$.

Since $\mathbb{T}$ is a measure space, the Bochner spaces $L^{2}\left(\mathbb{T} ; D_{0, \sigma}^{1,2}\left(\mathbb{R}^{3}\right)\right)$ and $L^{2}\left(\mathbb{T} ; D_{0}^{-1,2}\left(\mathbb{R}^{3}\right)^{3}\right)$ are well-defined; see Chapter 2.

We shall also need Bochner-Sobolev spaces of fractional order. We employ the Fourier transform to introduce these space. Recall that for any complex Hilbert space $H$ the Fourier transform $\mathcal{F}$ extends to an isomorphism

$$
\mathcal{F}: L^{2}(G ; H) \rightarrow L^{2}(\widehat{G} ; H),
$$

which is isometric with respect to the inner products in $L^{2}(G ; H)$ and $L^{2}(\widehat{G} ; H)$. We can now define the Bochner-Sobolev space with respect to the torus group $\mathbb{T}$. Recall that $\mathbb{T}$ is a locally compact abelian group, and that $\widehat{\mathbb{T}}$ can be identified with $\mathbb{Z}$. We can therefore define for $\alpha \in(0, \infty)$ the space

$$
W^{\alpha, 2}(\mathbb{T} ; H):=\left\{\left.u \in L^{2}(\mathbb{T} ; H)|| k\right|^{\alpha} \widehat{u}(k) \in L^{2}(\mathbb{Z} ; H)\right\}
$$

and norm

$$
\|u\|_{W^{\alpha, 2}(\mathbb{T} ; H)}:=\left(\sum_{k \in \mathbb{Z}}\left(1+|k|^{\alpha}\right)^{2}\|\widehat{u}(k)\|_{H}^{2}\right)^{1 / 2} .
$$

It is easy to see that $W^{\alpha, 2}(\mathbb{T} ; H)$ equipped with this norm is a Hilbert space.

If $H$ is a real Hilbert space, we define $W^{\alpha, 2}(0, T ; H)$ and $W^{\alpha, 2}(\mathbb{T} ; H)$ by using first the complexification of $H$, and subsequently restrict the elements to their real parts.

The weak formulation on the torus group requires smooth, solenoidal, test functions of compact support on the domain $\mathbb{R}^{3} \times \mathbb{T}$. As in Section 3.2.1, we use the quotient map (3.10) to define

$$
C^{\infty}\left(\mathbb{R}^{3} \times \mathbb{T}\right):=\left\{u: \mathbb{R}^{3} \times \mathbb{T} \rightarrow \mathbb{R} \mid \exists U \in C^{\infty}\left(\mathbb{R}^{3} \times \mathbb{R}\right): U=u \circ \pi\right\} .
$$

Introducing on $\mathbb{R}^{3} \times \mathbb{T}$ the canonical product topology, we can then define

$$
C_{0, \sigma}^{\infty}\left(\mathbb{R}^{3} \times \mathbb{T}\right):=\left\{u \in C^{\infty}\left(\mathbb{R}^{3} \times \mathbb{T}\right) \mid \operatorname{div} u=0, \operatorname{supp} u \text { is compact }\right\},
$$

which will serve as the space of test functions in the weak formulation. 


\subsubsection{Weak formulation}

We define a weak time-periodic solution to (6.1) as follows:

Definition 6.1.1. Let $f \in L^{2}\left(\mathbb{T} ; D_{0}^{-1,2}\left(\mathbb{R}^{3}\right)^{3}\right)$. A vector field $u \in L^{2}\left(\mathbb{T} ; D_{0, \sigma}^{1,2}\left(\mathbb{R}^{3}\right)\right)$ is called a weak time-periodic solution to (6.1) if

$$
\begin{aligned}
\forall \Phi \in & C_{0, \sigma}^{\infty}\left(\mathbb{R}^{3} \times \mathbb{T}\right): \\
& \int_{\mathbb{T}} \int_{\mathbb{R}^{3}}-u \cdot \partial_{t} \Phi+\nabla u: \nabla \Phi-\lambda \partial_{1} u \cdot \Phi+(u \cdot \nabla u) \cdot \Phi \mathrm{d} x \mathrm{~d} t=\int_{\mathbb{T}}\langle f, \Phi\rangle \mathrm{d} t .
\end{aligned}
$$

Remark 6.1.2. Recalling (2.2), we observe for $u$ and $\Phi$ as in Definition 6.1.1 that

$$
\int_{\mathbb{T}} \int_{\mathbb{R}^{3}}|(u \cdot \nabla u) \cdot \Phi| \mathrm{d} x \mathrm{~d} t \leq \int_{\mathbb{T}}\|u(t)\|_{6}\|\nabla u(t)\|_{2}\|\Phi(t)\|_{3} \mathrm{~d} t \leq \sup _{t \in \mathbb{T}}\|\Phi(t)\|_{3} \int_{\mathbb{T}}\|\nabla u(t)\|_{2}^{2} \mathrm{~d} t .
$$

Thus, the integral in (6.4) is well-defined.

Remark 6.1.3. In Section 6.5 we shall show existence of a pressure term $\mathfrak{p}$ that turns a weak solution in the sense of Definition 6.1.1 into a standard solution in the sense of distributions. Thus, (6.4) is an appropriate weak formulation. Definition 6.1.1 is equivalent to the definition of a weak time-periodic solution introduced in [25].

Remark 6.1.4. The function space $L^{2}\left(\mathbb{T} ; D_{0}^{-1,2}\left(\mathbb{R}^{3}\right)^{3}\right)$ used to describe the data $f$ in Definition 6.1.1 is chosen mainly because we shall later show existence of a weak solution for data in this class. The definition of a weak solution is valid also for data in other function spaces. The only requirement on the data is essentially that the right-hand side in (6.4) remains meaningful.

\subsection{Time-averaging projection}

We will show existence of a weak solution $u$ to (6.1) in the sense of Definition 6.1.1. In order to obtain better regularity than merely $u \in L^{2}\left(\mathbb{T} ; D_{0, \sigma}^{1,2}\left(\mathbb{R}^{3}\right)\right)$, we need to decompose $u$ into a steady-state part $u_{s}$ and a time-periodic part $u_{p}$, and analyze each part separately. For this purpose, we need the time-averaging projection $\mathcal{P}$ introduced in Section 3.5. We shall briefly reintroduce the projection in the setting of the Bochner spaces used in the weak setting.

For a Banach space $X$ we define the projection $\mathcal{P}$ on $L^{q}(\mathbb{T} ; X), q \in[1, \infty)$, by

$$
\mathcal{P}: L^{q}(\mathbb{T} ; X) \rightarrow L^{q}(\mathbb{T} ; X), \quad \mathcal{P} g:=\int_{\mathbb{T}} g(s) \mathrm{d} s,
$$

where the integral above is understood as a Bochner integral in $X$. As in the proof of Lemma 3.5.2, one can verify that $\mathcal{P}$ is continuous. Clearly, $\mathcal{P}$ is idempotent, and thus a continuous projection. Put

$$
\mathcal{P}_{\perp}: L^{q}(\mathbb{T} ; X) \rightarrow L^{q}(\mathbb{T} ; X), \quad \mathcal{P}_{\perp} g:=\mathrm{Id}-\mathcal{P} g .
$$




\section{Weak solutions}

Of course, $\mathcal{P}_{\perp}$ is also a continuous projection.

Observe that $\mathcal{P} g$ time-independent. More precisely, $\mathcal{P} L^{q}(\mathbb{T} ; X)=X$. Consequently, $\mathcal{P}$ induces that decomposition

$$
L^{q}(\mathbb{T} ; X)=X \oplus L_{\perp}^{q}(\mathbb{T} ; X),
$$

where $L_{\perp}^{q}(\mathbb{T} ; X)=\mathcal{P}_{\perp} L^{q}(\mathbb{T} ; X)$. For $u \in L^{q}(\mathbb{T} ; X)$ we will refer to $\mathcal{P} u$ as the steadystate part, and $\mathcal{P}_{\perp} u$ as the time-periodic part of $u$.

\subsection{Existence}

We shall now show existence of a weak time-periodic solution $u$ to (6.1). The proof uses a Galerkin approximation based on an appropriately chosen basis.

In addition to existence, it will be shown that $\mathcal{P}_{\perp} u \in L^{\infty}\left(\mathbb{T} ; L^{2}\left(\mathbb{R}^{3}\right)^{3}\right)$. The implications of this result are discussed in Remark 6.3.5. In contrast to the initial-value problem, it is in general not possible to show $u \in L^{\infty}\left(\mathbb{T} ; L^{2}\left(\mathbb{R}^{3}\right)^{3}\right)$ for a solution to the time-periodic problem. This issue is discussed further in Remark 6.3.3. The absence of a prescribed initial-value makes the task of showing $\mathcal{P}_{\perp} u \in L^{\infty}\left(\mathbb{T} ; L^{2}\left(\mathbb{R}^{3}\right)^{3}\right)$ in the time-periodic case more delicate than one would perhaps expect from the the relatively simple argument used to establish $u \in L^{\infty}\left(\mathbb{T} ; L^{2}\left(\mathbb{R}^{3}\right)^{3}\right)$ for the initial-value problem. We briefly discuss this matter in Remark 6.3.4.

We can now state and prove the main theorem on existence of weak solutions to (6.1).

Theorem 6.3.1. Let $f \in L^{2}\left(\mathbb{T} ; D_{0}^{-1,2}\left(\mathbb{R}^{3}\right)^{3}\right)$ and $\lambda \geq 0 .{ }^{1}$ There exists a weak timeperiodic solution $u \in L^{2}\left(\mathbb{T} ; D_{0, \sigma}^{1,2}\left(\mathbb{R}^{3}\right)\right)$ to (6.1) that satisfies

$$
\begin{aligned}
& \mathcal{P} u \in D_{0, \sigma}^{1,2}\left(\mathbb{R}^{3}\right), \\
& \mathcal{P}_{\perp} u \in L^{2}\left(\mathbb{T} ; D_{0, \sigma}^{1,2}\left(\mathbb{R}^{3}\right)\right) \cap L^{\infty}\left(\mathbb{T} ; L_{\sigma}^{2}\left(\mathbb{R}^{3}\right)\right) .
\end{aligned}
$$

Moreover,

$$
\forall \alpha \in\left[0, \frac{1}{3}\right): \quad \mathcal{P}_{\perp} u \in W^{\alpha, 2}\left(\mathbb{T} ; L_{\sigma}^{2}\left(\mathbb{R}^{3}\right)\right) .
$$

Proof. We let $\left\{\psi_{j}\right\}_{j \in \mathbb{N}} \subset C_{0, \sigma}^{\infty}\left(\mathbb{R}^{3}\right)^{3}$ be an orthonormal basis for the Hilbert space $W_{0, \sigma}^{1,2}\left(\mathbb{R}^{3}\right)$. Since $W_{0, \sigma}^{1,2}\left(\mathbb{R}^{3}\right)$ is separable and $C_{0, \sigma}^{\infty}\left(\mathbb{R}^{3}\right)$ dense herein, it is possible to find such a basis. For $j, k \in \mathbb{N}$ put

$$
\begin{aligned}
& \Psi_{j k}^{\#}: \mathbb{R}^{3} \times \mathbb{T} \rightarrow \mathbb{R}, \quad \Psi_{j k}^{\#}(x, t):=\frac{1}{\sqrt{2}} \psi_{j}(x) \cos \left(\frac{2 \pi}{\mathcal{T}} k t\right), \\
& \Psi_{j k}^{*}: \mathbb{R}^{3} \times \mathbb{T} \rightarrow \mathbb{R}, \quad \Psi_{j k}^{\#}(x, t):=\frac{1}{\sqrt{2}} \psi_{j}(x) \sin \left(\frac{2 \pi}{\mathcal{T}} k t\right), \\
& \Psi_{j}^{0}: \mathbb{R}^{3} \times \mathbb{T} \rightarrow \mathbb{R}, \quad \Psi_{j}^{0} k(x, t):=\psi_{j}(x) .
\end{aligned}
$$

\footnotetext{
${ }^{1}$ For convenience we consider only non-negative $\lambda$. The theorem holds for any $\lambda \in \mathbb{R}$.
} 
Clearly, $\left\{\Psi_{j k}^{\#}, \Psi_{j k}^{*}, \Psi_{j}^{0}\right\}_{k, j \in \mathbb{N}}$ is an orthonormal basis for $L^{2}\left(\mathbb{T} ; W_{0, \sigma}^{1,2}\left(\mathbb{R}^{3}\right)\right)$. For $n \geq m$ we let

$$
X_{n}^{m}:=\operatorname{span}\left\{\Psi_{j k}^{\#}, \Psi_{j k}^{*}, \Psi_{j}^{0} \mid j \leq m, k \leq n\right\}
$$

denote a finite linear span of these basis vectors. More precisely, we consider $X_{n}^{m}$ as a subspace of $L^{2}\left(\mathbb{T} ; W_{0, \sigma}^{1,2}\left(\mathbb{R}^{3}\right)\right)$.

We say that $U \in X_{n}^{m}$ is an approximate solution in $X_{n}^{m}$ to (6.1) if

$$
\begin{aligned}
& \forall \Phi \in X_{n}^{m}: \\
& \qquad \int_{\mathbb{T}} \int_{\mathbb{R}^{3}}-U \cdot \partial_{t} \Phi+\nabla U: \nabla \Phi-\lambda \partial_{1} U \cdot \Phi+(U \cdot \nabla U) \cdot \Phi \mathrm{d} x \mathrm{~d} t=\int_{\mathbb{T}}\langle f, \Phi\rangle \mathrm{d} t .
\end{aligned}
$$

We start by showing existence of such an approximate solution. Interestingly, we can use the same method that is employed in [18, Chapter IX.4] to treat the steady-state case. Let

$$
\forall \xi=\left(\xi^{\#}, \xi^{*}, \xi^{0}\right) \in \mathbb{R}^{m \times n} \times \mathbb{R}^{m \times n} \times \mathbb{R}^{m}: \quad U_{\xi}:=\sum_{j \leq m, k \leq n} \xi_{j k}^{\#} \Psi_{j k}^{\#}+\xi_{j k}^{*} \Psi_{j k}^{*}+\xi_{j}^{0} \Psi_{j}^{0} .
$$

Define the mapping

$$
P: \mathbb{R}^{m \times n} \times \mathbb{R}^{m \times n} \times \mathbb{R}^{m} \rightarrow \mathbb{R}^{m \times n} \times \mathbb{R}^{m \times n} \times \mathbb{R}^{m}, \quad P(\xi)=\left(P^{\#}(\xi), P^{*}(\xi), P^{0}(\xi)\right)
$$

by

$$
\begin{aligned}
& P_{j k}^{\#}(\xi):=\int_{\mathbb{T}} \int_{\mathbb{R}^{3}}\left(\partial_{t} U_{\xi}-\Delta U_{\xi}-\lambda \partial_{1} U_{\xi}+U_{\xi} \cdot \nabla U_{\xi}\right) \cdot \Psi_{j k}^{\#} \mathrm{~d} x \mathrm{~d} t-\int_{\mathbb{T}}\left\langle f, \Psi_{j k}^{\#}\right\rangle \mathrm{d} t, \\
& P_{j k}^{*}(\xi):=\int_{\mathbb{T}} \int_{\mathbb{R}^{3}}\left(\partial_{t} U_{\xi}-\Delta U_{\xi}-\lambda \partial_{1} U_{\xi}+U_{\xi} \cdot \nabla U_{\xi}\right) \cdot \Psi_{j k}^{*} \mathrm{~d} x \mathrm{~d} t-\int_{\mathbb{T}}\left\langle f, \Psi_{j k}^{*}\right\rangle \mathrm{d} t, \\
& P_{j}^{0}(\xi):=\int_{\mathbb{T}} \int_{\mathbb{R}^{3}}\left(\partial_{t} U_{\xi}-\Delta U_{\xi}-\lambda \partial_{1} U_{\xi}+U_{\xi} \cdot \nabla U_{\xi}\right) \cdot \Psi_{j}^{0} \mathrm{~d} x \mathrm{~d} t-\int_{\mathbb{T}}\left\langle f, \Psi_{j}^{0}\right\rangle \mathrm{d} t .
\end{aligned}
$$

Clearly, $P$ is continuous. Moreover,

$$
\begin{aligned}
P(\xi) \cdot \xi & =\int_{\mathbb{T}} \int_{\mathbb{R}^{3}} \partial_{t} U_{\xi} \cdot U_{\xi}-\Delta U_{\xi} \cdot U_{\xi}-\lambda \partial_{1} U_{\xi} \cdot U_{\xi}+\left(U_{\xi} \cdot \nabla U_{\xi}\right) \cdot U_{\xi} \mathrm{d} x \mathrm{~d} t-\int_{\mathbb{T}}\left\langle f, U_{\xi}\right\rangle \mathrm{d} t \\
& =\int_{\mathbb{T}} \int_{\mathbb{R}^{3}}\left|\nabla U_{\xi}\right|^{2} \mathrm{~d} x \mathrm{~d} t-\int_{\mathbb{T}}\left\langle f, U_{\xi}\right\rangle \mathrm{d} t \\
& \geq\left\|U_{\xi}\right\|_{L^{2}\left(\mathbb{T} ; D_{0, \sigma}^{1,2}\left(\mathbb{R}^{3}\right)\right)}^{2}-\|f\|_{L^{2}\left(\mathbb{T} ; D_{0}^{-1,2}\left(\mathbb{R}^{3}\right)\right)}\left\|U_{\xi}\right\|_{L^{2}\left(\mathbb{T} ; D_{0, \sigma}^{1,2}\left(\mathbb{R}^{3}\right)\right)} .
\end{aligned}
$$




\section{Weak solutions}

Since the family of vectors $\left\{\Psi_{j k}^{\#}, \Psi_{j k}^{*}, \Psi_{j}^{0} \mid j \leq m, k \leq n\right\}$ is linearly independent in the space $L^{2}\left(\mathbb{T} ; W_{0, \sigma}^{1,2}\left(\mathbb{R}^{3}\right)\right)$, it is also linearly independent as a family of vectors in the space $L^{2}\left(\mathbb{T} ; D_{0, \sigma}^{1,2}\left(\mathbb{R}^{3}\right)\right)$. Consequently, there are constants $A, B>0$ such that

$$
\forall \xi \in \mathbb{R}^{m \times n} \times \mathbb{R}^{m \times n} \times \mathbb{R}^{m}: A|\xi| \leq\left\|U_{\xi}\right\|_{L^{2}\left(\mathbb{T} ; D_{0, \sigma}^{1,2}\left(\mathbb{R}^{3}\right)\right)} \leq B|\xi| .
$$

It follows that

$$
P(\xi) \cdot \xi \geq A^{2}|\xi|^{2}-B\|f\|_{L^{2}\left(\mathbb{T} ; D_{0}^{-1,2}\left(\mathbb{R}^{3}\right)\right)}|\xi|,
$$

whence $P(\xi) \cdot \xi>0$ for all $\xi \in \mathbb{R}^{m \times n} \times \mathbb{R}^{m \times n} \times \mathbb{R}^{m}$ with $|\xi|=2 \frac{B}{A^{2}}\|f\|$. Thus, $P$ satisfies the condition in [18, Lemma VIII.3.1] from which the existence of $\xi_{0} \in \mathbb{R}^{m \times n} \times \mathbb{R}^{m \times n} \times \mathbb{R}^{m}$ with the property

$$
P\left(\xi_{0}\right)=0
$$

then follows. We conclude that $U_{\xi_{0}}$ is an approximate solution in $X_{n}^{m}$. We put $U_{n}^{m}:=$ $U_{\xi_{0}}$. By a similar computation as in (6.12), we find

$$
0=P\left(\xi_{0}\right) \cdot \xi_{0}=\int_{\mathbb{T}} \int_{\mathbb{R}^{3}}\left|\nabla U_{n}^{m}\right|^{2} \mathrm{~d} x \mathrm{~d} t-\int_{\mathbb{T}}\left\langle f, U_{n}^{m}\right\rangle \mathrm{d} t,
$$

and thus conclude

$$
\left\|U_{n}^{m}\right\|_{L^{2}\left(\mathbb{T} ; D_{0, \sigma}^{1,2}\left(\mathbb{R}^{3}\right)\right)} \leq\|f\|_{L^{2}\left(\mathbb{T} ; D_{0}^{-1,2}\left(\mathbb{R}^{3}\right)\right)} .
$$

Summarizing, we have found for all $m, n \in \mathbb{N}$ an approximate solution $U_{n}^{m} \in X_{n}^{m}$ to (6.1) that satisfies (6.14).

In the next step, we seek to establish a similar bound on the norm of $\mathcal{P}_{\perp} U_{n}^{m}$ in the space $W^{\alpha, 2}\left(\mathbb{T} ; L_{\sigma}^{2}\left(\mathbb{R}^{3}\right)\right)$. For this purpose, it will be convenient to express $U_{n}^{m}(x, \cdot)$ in terms of the basis $\left\{\mathrm{e}^{i \frac{2 \pi}{T} k t} \mid k \in \mathbb{Z}\right\}$ of the complex Hilbert space $L^{2}(\mathbb{T} ; \mathbb{C})$. More precisely, we put

$$
\forall k \in \mathbb{Z}: \quad u_{k}(x):=\int_{\mathbb{T}} U_{n}^{m}(x, t) \mathrm{e}^{-i \frac{2 \pi}{\mathcal{T}} k t} \mathrm{~d} t \in C_{0, \sigma}^{\infty}\left(\mathbb{R}^{3}\right) .
$$

Clearly, $u_{k}=0$ for all $|k|>n$. Since $\left\{u_{k}(x) \mid k \in \mathbb{Z}\right\}$, are the Fourier coefficients of $U_{n}^{m}(x, \cdot)$, we have

$$
U_{n}^{m}(x, t)=\sum_{k \in \mathbb{Z}} u_{k}(x) \mathrm{e}^{i \frac{2 \pi}{\mathcal{T}} k t}=\sum_{|k| \leq n} u_{k}(x) \mathrm{e}^{i \frac{2 \pi}{\mathcal{T}} k t} .
$$

Observe that

$$
\begin{aligned}
& \mathcal{P} U_{n}^{m}(x, t)=u_{0}(x), \\
& \mathcal{P}_{\perp} U_{n}^{m}(x, t)=\sum_{k \in \mathbb{Z} \backslash\{0\}} u_{k}(x) \mathrm{e}^{i \frac{2 \pi}{\mathcal{T}} k t}=\sum_{|k| \leq n, k \neq 0} u_{k}(x) \mathrm{e}^{i \frac{2 \pi}{\mathcal{T}} k t} .
\end{aligned}
$$


For any $l \in \mathbb{Z} \backslash\{0\}$ and $\gamma \in \mathbb{R}, \gamma>0$ we will now $\operatorname{use}^{2}(-i) \operatorname{sgn}(l)|l|^{-\gamma} \overline{u_{l}} \mathrm{e}^{-i \frac{2 \pi}{T} l t}$ as a test function in the weak formulation (6.11). We therefore compute

$$
\begin{aligned}
\int_{\mathbb{T}} \int_{\mathbb{R}^{3}} \partial_{t} U_{n}^{m} & \cdot(-i) \operatorname{sgn}(l)|l|^{-\gamma} \overline{u_{l}} \mathrm{e}^{-i \frac{2 \pi}{\mathcal{T}} l t} \mathrm{~d} x \mathrm{~d} t \\
& =\int_{\mathbb{T}} \int_{\mathbb{R}^{3}}\left(\sum_{k \in \mathbb{Z}} i \frac{2 \pi}{\mathcal{T}} k u_{k}(x) \mathrm{e}^{i \frac{2 \pi}{\mathcal{T}} k t}\right) \cdot(-i) \operatorname{sgn}(l)|l|^{-\gamma} \overline{u_{l}} \mathrm{e}^{-i \frac{2 \pi}{\mathcal{T}} l t} \mathrm{~d} x \mathrm{~d} t \\
& =\int_{\mathbb{R}^{3}} \frac{2 \pi}{\mathcal{T}} l \operatorname{sgn}(l)|l|^{-\gamma} u_{l} \overline{u_{l}} \mathrm{~d} x \\
& =\frac{2 \pi}{\mathcal{T}}|l|^{1-\gamma} \int_{\mathbb{R}^{3}}\left|u_{l}\right|^{2} \mathrm{~d} x .
\end{aligned}
$$

Similarly, we compute

$$
\int_{\mathbb{T}} \int_{\mathbb{R}^{3}} \nabla U_{n}^{m}: \nabla\left[(-i) \operatorname{sgn}(l)|l|^{-\gamma} \overline{u_{l}} \mathrm{e}^{-i \frac{2 \pi}{T} l t}\right] \mathrm{d} x \mathrm{~d} t=(-i) \operatorname{sgn}(l)|l|^{-\gamma} \int_{\mathbb{R}^{3}}\left|\nabla u_{l}\right|^{2} \mathrm{~d} x,
$$

and

$$
\int_{\mathbb{T}} \int_{\mathbb{R}^{3}} \lambda \partial_{1} U_{n}^{m} \cdot(-i) \operatorname{sgn}(l)|l|^{-\gamma} \overline{u_{l}} \mathrm{e}^{-i \frac{2 \pi}{\mathcal{T}} l t} \mathrm{~d} x \mathrm{~d} t=(-i) \operatorname{sgn}(l)|l|^{-\gamma} \lambda \int_{\mathbb{R}^{3}} \partial_{1} u_{l} \overline{u_{l}} \mathrm{~d} x .
$$

Since $U_{n}^{m}$ is a real function, it follows that $\overline{u_{k}}=u_{-k}$. Consequently

$$
\begin{aligned}
& \int_{\mathbb{T}} \int_{\mathbb{R}^{3}}\left(U_{n}^{m} \cdot \nabla U_{n}^{m}\right) \cdot(-i) \operatorname{sgn}(l)|l|^{-\gamma} \overline{u_{l}} \mathrm{e}^{-i \frac{2 \pi}{\mathcal{T}} l t} \mathrm{~d} x \mathrm{~d} t \\
& =\int_{\mathbb{T}} \int_{\mathbb{R}^{3}}\left(\left(\sum_{|k| \leq n} u_{k}(x) \mathrm{e}^{i \frac{2 \pi}{\mathcal{T}} k t}\right) \cdot \nabla\left(\sum_{|h| \leq n} u_{h}(x) \mathrm{e}^{i \frac{2 \pi}{\mathcal{T}} h t}\right)\right) \cdot(-i) \operatorname{sgn}(l)|l|^{-\gamma} \overline{u_{l}} \mathrm{e}^{-i \frac{2 \pi}{\mathcal{T}} l t} \mathrm{~d} x \mathrm{~d} t \\
& =\int_{\mathbb{T}} \int_{\mathbb{R}^{3}} \sum_{|k|,|h| \leq n}\left(u_{k} \cdot \nabla u_{h}\right) \cdot\left((-i) \operatorname{sgn}(l)|l|^{-\gamma} u_{-l} \mathrm{e}^{i \frac{2 \pi}{\mathcal{T}}(k+h-l) t}\right) \mathrm{d} x \mathrm{~d} t \\
& =(-i) \operatorname{sgn}(l)|l|^{-\gamma} \int_{\mathbb{R}^{3}} \sum_{|k| \leq n}\left(u_{k} \cdot \nabla u_{l-k}\right) \cdot u_{-l} \mathrm{~d} x .
\end{aligned}
$$

For $|l| \leq n$ it is easy to see that the function $(-i) \operatorname{sgn}(l)|l|^{-\gamma} \overline{u_{l}} \mathrm{e}^{-i \frac{2 \pi}{\mathcal{T}} l t}$ belongs to the complexification $X_{n}^{m} \oplus i X_{n}^{m}$ of $X_{n}^{m}$. Consequently, we can use it as a test function in

\footnotetext{
$\overline{{ }^{2}} \operatorname{By} \operatorname{sgn}(l)$ we denote the sign of $l$, and by $\bar{u}$ the complex conjugate of $u$.
} 


\section{Weak solutions}

the weak formulation (6.11). From (6.11) and (6.18)-(6.21) it therefore follows that

$$
\begin{aligned}
\frac{2 \pi}{\mathcal{T}}|l|^{1-\gamma} \int_{\mathbb{R}^{3}}\left|u_{l}\right|^{2} \mathrm{~d} x= & -(-i) \operatorname{sgn}(l)|l|^{-\gamma} \int_{\mathbb{R}^{3}}\left|\nabla u_{l}\right|^{2} \mathrm{~d} x \\
& +(-i) \operatorname{sgn}(l)|l|^{-\gamma} \lambda \int_{\mathbb{R}^{3}} \partial_{1} u_{l} \overline{u_{l}} \mathrm{~d} x \\
& -(-i) \operatorname{sgn}(l)|l|^{-\gamma} \int_{\mathbb{R}^{3}} \sum_{|k| \leq n}\left(u_{k} \cdot \nabla u_{l-k}\right) \cdot u_{-l} \mathrm{~d} x \\
& +(-i) \operatorname{sgn}(l)|l|^{-\gamma} \int_{\mathbb{T}}\left\langle f, \overline{u_{l}}\right\rangle \mathrm{e}^{-i \frac{2 \pi}{\mathcal{T}} l t} \mathrm{~d} t .
\end{aligned}
$$

We shall estimate the terms on the right-hand side above. To estimate the second term, we employ first Hölder's and then Young's inequality to obtain

$$
\begin{aligned}
\left.|| l\right|^{-\gamma} \lambda \int_{\mathbb{R}^{3}} \partial_{1} u_{l} \overline{u_{l}} \mathrm{~d} x \mid & \leq|l|^{-\gamma} \lambda\left(\int_{\mathbb{R}^{3}}\left|\nabla u_{l}\right|^{2} \mathrm{~d} x\right)^{\frac{1}{2}}\left(\int_{\mathbb{R}^{3}}\left|u_{l}\right|^{2} \mathrm{~d} x\right)^{\frac{1}{2}} \\
& \leq|l|^{-\gamma}\left(\frac{1}{2} \frac{2 \pi}{\mathcal{T}} \int_{\mathbb{R}^{3}}\left|u_{l}\right|^{2} \mathrm{~d} x+c_{1} \int_{\mathbb{R}^{3}}\left|\nabla u_{l}\right|^{2} \mathrm{~d} x\right) \\
& \leq|l|^{1-\gamma} \frac{1}{2} \frac{2 \pi}{\mathcal{T}} \int_{\mathbb{R}^{3}}\left|u_{l}\right|^{2} \mathrm{~d} x+c_{1} \int_{\mathbb{R}^{3}}\left|\nabla u_{l}\right|^{2} \mathrm{~d} x
\end{aligned}
$$

where $c_{1}=c_{1}(\lambda, \mathcal{T})$. Recalling (2.2), we estimate, employing Hölder's inequality, the third term on the right-hand side in (6.22) by

$$
\begin{aligned}
& \left.|| l\right|^{-\gamma} \int_{\mathbb{R}^{3}} \sum_{|k| \leq n}\left(u_{k} \cdot \nabla u_{l-k}\right) \cdot u_{-l} \mathrm{~d} x \mid \\
& \quad \leq|l|^{-\gamma} \sum_{|k| \leq n}\left(\int_{\mathbb{R}^{3}}\left|u_{k}\right|^{6} \mathrm{~d} x\right)^{\frac{1}{6}}\left(\int_{\mathbb{R}^{3}}\left|\nabla u_{l-k}\right|^{2} \mathrm{~d} x\right)^{\frac{1}{2}}\left(\int_{\mathbb{R}^{3}}\left|u_{l}\right|^{3} \mathrm{~d} x\right)^{\frac{1}{3}} \\
& \quad \leq c_{2}|l|^{-\gamma} \sum_{|k| \leq n}\left(\int_{\mathbb{R}^{3}}\left|\nabla u_{k}\right|^{2} \mathrm{~d} x\right)^{\frac{1}{2}}\left(\int_{\mathbb{R}^{3}}\left|\nabla u_{l-k}\right|^{2} \mathrm{~d} x\right)^{\frac{1}{2}}\left(\int_{\mathbb{R}^{3}}\left|u_{l}\right|^{3} \mathrm{~d} x\right)^{\frac{1}{3}} \\
& \quad \leq c_{3}|l|^{-\gamma}\left(\int_{\mathbb{R}^{3}}\left|u_{l}\right|^{3} \mathrm{~d} x\right)^{\frac{1}{3}}\left(\sum_{|k| \leq n} \int_{\mathbb{R}^{3}}\left|\nabla u_{k}\right|^{2} \mathrm{~d} x\right)^{\frac{1}{2}}\left(\sum_{|k| \leq n} \int_{\mathbb{R}^{3}}\left|\nabla u_{l-k}\right|^{2} \mathrm{~d} x\right)^{\frac{1}{2}} .
\end{aligned}
$$

Employing Lemma 2.3.1 to estimate the first integral on the right-hand side above, we 
further obtain

$$
\begin{aligned}
& \left.|| l\right|^{-\gamma} \int_{\mathbb{R}^{3}} \sum_{|k| \leq n}\left(u_{l-k} \cdot \nabla u_{k}\right) \cdot u_{-l} \mathrm{~d} x \mid \\
& \quad \leq c_{4}|l|^{-\gamma}\left(\int_{\mathbb{R}^{3}}\left|u_{l}\right|^{2} \mathrm{~d} x\right)^{\frac{1}{4}}\left(\int_{\mathbb{R}^{3}}\left|\nabla u_{l}\right|^{2} \mathrm{~d} x\right)^{\frac{1}{4}}\left(\sum_{k \in \mathbb{Z}} \int_{\mathbb{R}^{3}}\left|\nabla u_{k}\right|^{2} \mathrm{~d} x\right),
\end{aligned}
$$

whence, by Plancherel's equality,

$$
\begin{aligned}
& \left.|| l\right|^{-\gamma} \int_{\mathbb{R}^{3}} \sum_{|k| \leq n}\left(u_{k} \cdot \nabla u_{l-k}\right) \cdot u_{-l} \mathrm{~d} x \mid \\
& \quad \leq c_{5}|l|^{-\gamma}\left(\int_{\mathbb{R}^{3}}\left|u_{l}\right|^{2} \mathrm{~d} x\right)^{\frac{1}{4}}\left(\int_{\mathbb{R}^{3}}\left|\nabla u_{l}\right|^{2} \mathrm{~d} x\right)^{\frac{1}{4}}\left(\int_{\mathbb{T}} \int_{\mathbb{R}^{3}}\left|\nabla U_{n}^{m}\right|^{2} \mathrm{~d} x \mathrm{~d} t\right) .
\end{aligned}
$$

We can estimate the last term on the right-hand side in (6.22) by

$$
\left.|| l\right|^{-\gamma} \int_{\mathbb{T}}\left\langle f, \overline{u_{l}}\right\rangle \mathrm{e}^{-i \frac{2 \pi}{\mathcal{T}} l t} \mathrm{~d} t \mid \leq\left\|f_{l}\right\|_{D_{0}^{-1,2}\left(\mathbb{R}^{3}\right)}^{2}+\left\|\nabla u_{l}\right\|_{2}^{2}
$$

where $f_{l}$ denotes the $l$ 'th Fourier coefficient of $f$. Collecting (6.22)-(6.25) and recalling (6.14), we can now deduce

$$
\begin{aligned}
& |l|^{1-\gamma} \int_{\mathbb{R}^{3}}\left|u_{l}\right|^{2} \mathrm{~d} x \\
& \quad \leq c_{6}\left(\int_{\mathbb{R}^{3}}\left|\nabla u_{l}\right|^{2} \mathrm{~d} x+|l|^{-\gamma}\left(\int_{\mathbb{R}^{3}}\left|u_{l}\right|^{2} \mathrm{~d} x\right)^{\frac{1}{4}}\left(\int_{\mathbb{R}^{3}}\left|\nabla u_{l}\right|^{2} \mathrm{~d} x\right)^{\frac{1}{4}}+\left\|f_{l}\right\|_{D_{0}^{-1,2}\left(\mathbb{R}^{3}\right)}^{2}\right),
\end{aligned}
$$

where $c_{6}=c_{6}(\|f\|, \mathcal{T}, \lambda)$. Finally, we employ Young's inequality to estimate

$$
\begin{aligned}
|l|^{-\gamma}\left(\int_{\mathbb{R}^{3}}\left|u_{l}\right|^{2} \mathrm{~d} x\right)^{\frac{1}{4}} & \left(\int_{\mathbb{R}^{3}}\left|\nabla u_{l}\right|^{2} \mathrm{~d} x\right)^{\frac{1}{4}} \\
& =|l|^{-\gamma-\frac{1}{4}(1-\gamma)}\left(\int_{\mathbb{R}^{3}}|l|^{1-\gamma}\left|u_{l}\right|^{2} \mathrm{~d} x\right)^{\frac{1}{4}}\left(\int_{\mathbb{R}^{3}}\left|\nabla u_{l}\right|^{2} \mathrm{~d} x\right)^{\frac{1}{4}} \\
& \leq c_{7}|l|^{-2\left(\frac{3}{4} \gamma+\frac{1}{4}\right)}+\frac{1}{2 c_{6}} \int_{\mathbb{R}^{3}}|l|^{1-\gamma}\left|u_{l}\right|^{2} \mathrm{~d} x+c_{8} \int_{\mathbb{R}^{3}}\left|\nabla u_{l}\right|^{2} \mathrm{~d} x
\end{aligned}
$$

and conclude

$$
|l|^{1-\gamma} \int_{\mathbb{R}^{3}}\left|u_{l}\right|^{2} \mathrm{~d} x \leq c_{9}\left(\int_{\mathbb{R}^{3}}\left|\nabla u_{l}\right|^{2} \mathrm{~d} x+|l|^{-2\left(\frac{3}{4} \gamma+\frac{1}{4}\right)}+\left\|f_{l}\right\|_{D_{0}^{-1,2}\left(\mathbb{R}^{3}\right)}^{2}\right) .
$$




\section{Weak solutions}

We have derived the above inequality for $l \in \mathbb{Z} \backslash\{0\}$ with $|l| \leq n$. However, since $u_{l}=0$ for $|l|>n$, the above inequality holds for all $l \in \mathbb{Z} \backslash\{0\}$. We will now sum both sides over $l$. For $\gamma>\frac{1}{3}$ we have

$$
-2\left(\frac{3}{4} \gamma+\frac{1}{4}\right)<-1
$$

whence, using again Plancherel's equality, it follows that

$$
\begin{aligned}
\sum_{l \in \mathbb{Z} \backslash\{0\}}|l|^{1-\gamma}\left\|u_{l}\right\|_{L^{2}\left(\mathbb{R}^{3}\right)}^{2} \mathrm{~d} x & \leq c_{10}\left(\sum_{l \in \mathbb{Z}_{\mathbb{R}^{3}}}\left|\nabla u_{l}\right|^{2} \mathrm{~d} x+1+\|f\|_{L^{2}\left(\mathbb{T} ; D_{0}^{-1,2}\left(\mathbb{R}^{3}\right)\right)}^{2}\right) \\
& =c_{10}\left(\int_{\mathbb{T}} \int_{\mathbb{R}^{3}}\left|\nabla U_{n}^{m}\right|^{2} \mathrm{~d} x \mathrm{~d} t+1+\|f\|_{L^{2}\left(\mathbb{T} ; D_{0}^{-1,2}\left(\mathbb{R}^{3}\right)\right)}^{2}\right),
\end{aligned}
$$

where $c_{10}=c_{10}(\|f\|, \gamma, \mathcal{T}, \lambda)$. Recalling (6.14), we deduce

$$
\forall \gamma \in\left(\frac{1}{3}, \infty\right): \quad \sum_{l \in \mathbb{Z} \backslash\{0\}}|l|^{1-\gamma}\left\|u_{l}\right\|_{L^{2}\left(\mathbb{R}^{3}\right)}^{2} \mathrm{~d} x \leq c_{10},
$$

with $c_{10}$ independent on $m$ and $n$. Recalling (6.17), we conclude for all $\alpha \in\left[0, \frac{1}{3}\right)$ that $\mathcal{P}_{\perp} U_{n}^{m} \in W^{\alpha, 2}\left(\mathbb{T} ; L_{\sigma}^{2}\left(\mathbb{R}^{3}\right)\right)$ with

$$
\forall \alpha \in\left[0, \frac{1}{3}\right): \quad\left\|\mathcal{P}_{\perp} U_{n}^{m}\right\|_{W^{\alpha, 2}\left(\mathbb{T} ; L_{\sigma}^{2}\left(\mathbb{R}^{3}\right)\right)} \leq c_{11},
$$

where $c_{11}=c_{11}(\alpha,\|f\|, \mathcal{T}, \lambda)$ is independent on $m$ and $n$.

In the next step of the proof, we wish to establish an estimate of the approximate solution in the $L^{\infty}\left(\mathbb{T} ; L^{2}\left(\mathbb{R}^{3}\right)^{3}\right)$-norm. For this purpose, we choose again an appropriate test function and utilize that $U_{n}^{m}$ satisfies (6.11). We shall also need to pass to the limit $n \rightarrow \infty$. We therefore denote now the Fourier coefficients of $U_{n}^{m}(x, \cdot)$ by $\left\{u_{k}^{n}\right\}_{k \in \mathbb{Z}}$. Thus, we have

$$
U_{n}^{m}(x, t)=\sum_{k \in \mathbb{Z}} u_{k}^{n}(x) \mathrm{e}^{i \frac{2 \pi}{\mathcal{T}} k t}=\sum_{|k| \leq n} u_{k}^{n}(x) \mathrm{e}^{i \frac{2 \pi}{\mathcal{T}} k t}
$$

We choose for $j, l \in \mathbb{Z}$ with $|j|,|l| \leq n$ the function $u_{j}^{n} \mathrm{e}^{-i \frac{2 \pi}{T} l t} \in X_{n}^{m} \oplus i X_{n}^{m}$ as test function in (6.11) and obtain

$$
\begin{aligned}
& \int_{\mathbb{T}} \int_{\mathbb{R}^{3}}-U_{n}^{m} \cdot\left(-i \frac{2 \pi}{\mathcal{T}} l u_{j}^{n} \mathrm{e}^{-i \frac{2 \pi}{\mathcal{T}} l t}\right)+\nabla U_{n}^{m}: \nabla u_{j}^{n} \mathrm{e}^{-i \frac{2 \pi}{\mathcal{T}} l t} \\
& \quad-\lambda \partial_{1} U_{n}^{m} \cdot u_{j}^{n} \mathrm{e}^{-i \frac{2 \pi}{\mathcal{T}} l t}+\left(U_{n}^{m} \cdot \nabla U_{n}^{m}\right) \cdot u_{j}^{n} \mathrm{e}^{-i \frac{2 \pi}{\mathcal{T}} l t} \mathrm{~d} x \mathrm{~d} t=\int_{\mathbb{T}}\left\langle f, u_{j}^{n}\right\rangle \mathrm{e}^{-i \frac{2 \pi}{\mathcal{T}} l t} \mathrm{~d} t .
\end{aligned}
$$


Inserting the Fourier series (6.29) for $U_{n}^{m}$ in the above identity, we find

$$
\begin{aligned}
\int_{\mathbb{R}^{3}} i \frac{2 \pi}{\mathcal{T}} l u_{l}^{n} u_{j}^{n}+\nabla u_{l}^{n}: \nabla u_{j}^{n}-\lambda \partial_{1} u_{l}^{n} \cdot u_{j}^{n}+\sum_{|k| \leq n}\left(u_{l-k}^{n} \cdot \nabla u_{k}^{n}\right) \cdot u_{j}^{n} \mathrm{~d} x & \\
& =\int_{\mathbb{T}}\left\langle f, u_{j}^{n}\right\rangle \mathrm{e}^{-i \frac{2 \pi}{\mathcal{T}} l s} \mathrm{~d} s .
\end{aligned}
$$

Multiplying both sides above with $\mathrm{e}^{i \frac{2 \pi}{T}(l+j) t}$ and summing over $j$ and $l$, we further deduce

$$
\begin{aligned}
\sum_{|j|,|l| \leq n} \int_{\substack{j \neq 0 \\
\mathbb{R}^{3}}} i \frac{2 \pi}{\mathcal{T}} l u_{l}^{n} u_{j}^{n} \mathrm{e}^{i \frac{2 \pi}{\mathcal{T}}(l+j) t} \mathrm{~d} x= & \sum_{\substack{|j|,|l| \leq n \\
j \neq 0}} \int_{\mathbb{R}^{3}}-\nabla u_{l}^{n}: \nabla u_{j}^{n} \mathrm{e}^{i \frac{2 \pi}{\mathcal{T}}(l+j) t} \mathrm{~d} x \\
& +\sum_{\substack{|j|,|l| \leq n \\
j \neq 0}} \int_{\mathbb{R}^{3}} \lambda \partial_{1} u_{l}^{n} \cdot u_{j}^{n} \mathrm{e}^{i \frac{2 \pi}{\mathcal{T}}(l+j) t} \mathrm{~d} x \\
& -\sum_{\substack{|k|,|j|,|l| \leq n \\
j \neq 0}} \int_{\mathbb{R}^{3}}\left(u_{l-k}^{n} \cdot \nabla u_{k}^{n}\right) \cdot u_{j}^{n} \mathrm{e}^{i \frac{2 \pi}{\mathcal{T}}(l+j) t} \mathrm{~d} x \\
& +\sum_{\substack{|j|,|l| \leq n \\
j \neq 0}} \int_{\mathbb{T}}\left\langle f, u_{j}^{n}\right\rangle \mathrm{e}^{-i \frac{2 \pi}{\mathcal{T}} l s} \mathrm{~d} s \mathrm{e}^{i \frac{2 \pi}{\mathcal{T}}(l+j) t} .
\end{aligned}
$$

In the sums in (6.30) we recognize familiar quantities. For example, we compute, recalling that $\mathcal{P}_{\perp} U_{n}^{m}$ is a real function,

$$
\begin{aligned}
\partial_{t} \int_{\mathbb{R}^{3}}\left|\mathcal{P}_{\perp} U_{n}^{m}(x, t)\right|^{2} \mathrm{~d} x= & \partial_{t} \int_{\mathbb{R}^{3}} \sum_{\substack{|l|,|j| \leq n \\
l, j \neq 0}} u_{l}^{n} u_{j}^{n} \mathrm{e}^{i \frac{2 \pi}{\mathcal{T}}(l+j) t} \mathrm{~d} x \\
= & \sum_{\substack{|l|,|j| \leq n \\
l, j \neq 0}} \int_{\mathbb{R}^{3}} i \frac{2 \pi}{\mathcal{T}}(l+j) u_{l}^{n} u_{j}^{n} \mathrm{e}^{i \frac{2 \pi}{\mathcal{T}}(l+j) t} \mathrm{~d} x \\
= & \sum_{\substack{|l|,|j| \leq n \\
j \neq 0}} \int_{\mathbb{R}^{3}} i \frac{2 \pi}{\mathcal{T}} l u_{l}^{n} u_{j}^{n} \mathrm{e}^{i \frac{2 \pi}{\mathcal{T}}(l+j) t} \mathrm{~d} x \\
& +\sum_{\substack{|l|,|j| \leq n \\
l \neq 0}} \int_{\mathbb{R}^{3}} i \frac{2 \pi}{\mathcal{T}} j u_{l}^{n} u_{j}^{n} \mathrm{e}^{i \frac{2 \pi}{\mathcal{T}}(l+j) t} \mathrm{~d} x \\
= & 2 \sum_{\substack{|l|,|j| \leq n \\
j \neq 0}} \int_{\mathbb{R}^{3}} i \frac{2 \pi}{\mathcal{T}} l u_{l}^{n} u_{j}^{n} \mathrm{e}^{i \frac{2 \pi}{\mathcal{T}}(l+j) t} \mathrm{~d} x .
\end{aligned}
$$

We have hereby identified the left-hand side in (6.30) with $\frac{1}{2} \partial_{t} \int_{\mathbb{R}^{3}}\left|\mathcal{P}_{\perp} U^{m}(x, t)\right|^{2} \mathrm{~d} x$. Similar identifications can be made for the terms on the right-hand side as well. We immediately see that

$$
\sum_{\substack{|l|,|j| \leq n \\ j \neq 0}} \int_{\mathbb{R}^{3}} \nabla u_{l}^{n}: \nabla u_{j}^{n} \mathrm{e}^{-i \frac{2 \pi}{\mathcal{T}}(l+j) t} \mathrm{~d} x=\int_{\mathbb{R}^{3}} \nabla U_{n}^{m}: \nabla \mathcal{P}_{\perp} U_{n}^{m} \mathrm{~d} x
$$


and

$$
\sum_{\substack{|l|,|j| \leq n \\ j \neq 0}} \int_{\mathbb{R}^{3}} \lambda \partial_{1} u_{l}^{n} \cdot u_{j}^{n} \mathrm{e}^{-i \frac{2 \pi}{\mathcal{T}}(l+j) t} \mathrm{~d} x=\int_{\mathbb{R}^{3}} \lambda \partial_{1} U_{n}^{m} \cdot \mathcal{P}_{\perp} U_{n}^{m} \mathrm{~d} x .
$$

We also see that

$$
\sum_{\substack{|l|,|j| \leq n \\ j \neq 0}} \int_{\mathbb{T}}\left\langle f, u_{j}^{n}\right\rangle \mathrm{e}^{-i \frac{2 \pi}{\mathcal{T}} l s} \mathrm{~d} s \mathrm{e}^{i \frac{2 \pi}{\mathcal{T}}(l+j) t}=\sum_{|l| \leq n}\left\langle f_{l}, \mathcal{P}_{\perp} U_{n}^{m}\right\rangle \mathrm{e}^{i \frac{2 \pi}{\mathcal{T}} l t},
$$

where $f_{l}$ denotes the $l$ 'th Fourier coefficient of $f$. Combining (6.30)-(6.34), we conclude that

$$
\begin{aligned}
\frac{1}{2} \partial_{t} \int_{\mathbb{R}^{3}}\left|\mathcal{P}_{\perp} U_{n}^{m}(x, t)\right|^{2} \mathrm{~d} x= & -\int_{\mathbb{R}^{3}} \nabla U_{n}^{m}: \nabla \mathcal{P}_{\perp} U_{n}^{m} \mathrm{~d} x+\int_{\mathbb{R}^{3}} \lambda \partial_{1} U_{n}^{m} \cdot \mathcal{P}_{\perp} U_{n}^{m} \mathrm{~d} x \\
& -\sum_{\substack{|k|,|j|,|l| \leq n \\
j \neq 0}} \int_{\mathbb{R}^{3}}\left(u_{l-k}^{n} \cdot \nabla u_{k}^{n}\right) \cdot u_{j}^{n} \mathrm{e}^{i \frac{2 \pi}{\mathcal{T}}(l+j) t} \mathrm{~d} x \\
& +\sum_{|l| \leq n}\left\langle f_{l}, \mathcal{P}_{\perp} U_{n}^{m}\right\rangle \mathrm{e}^{i \frac{2 \pi}{\mathcal{T}} l t} .
\end{aligned}
$$

Observe that we now have a pointwise identity for $\partial_{t} \int_{\mathbb{R}^{3}}\left|\mathcal{P}_{\perp} U_{n}^{m}(x, t)\right|^{2} \mathrm{~d} x$. To capitalize on this information, we compute for $h \in \mathbb{Z}$ the $h$ 'th Fourier coefficient on both sides in (6.35) to find that

$$
\begin{aligned}
\int_{\mathbb{T}}\left(-i \frac{2 \pi}{\mathcal{T}} h\right)\left(\frac{1}{2} \int_{\mathbb{R}^{3}}\left|\mathcal{P}_{\perp} U_{n}^{m}(x, t)\right|^{2} \mathrm{~d} x\right) \mathrm{e}^{-i \frac{2 \pi}{T} h t} \mathrm{~d} t \\
=\int_{\mathbb{T}}\left(\int_{\mathbb{R}^{3}}-\nabla U_{n}^{m}: \nabla \mathcal{P}_{\perp} U_{n}^{m} \mathrm{~d} x+\lambda \partial_{1} U_{n}^{m} \cdot \mathcal{P}_{\perp} U_{n}^{m} \mathrm{~d} x\right) \mathrm{e}^{-i \frac{2 \pi}{\mathcal{T}} h t} \mathrm{~d} t \\
\quad-\sum_{\substack{|k|,|j| \leq n \\
j \neq 0,|h-j| \leq n}} \int_{\mathbb{R}^{3}}\left(u_{h-j-k}^{n} \cdot \nabla u_{k}^{n}\right) \cdot u_{j}^{n} \mathrm{~d} x+\left\langle f_{h}, \mathcal{P}_{\perp} U_{n}^{m}\right\rangle .
\end{aligned}
$$

For the purpose of expressing the sum on the right-hand side above in terms of more well-known quantities, we shall pass to the limit $n \rightarrow \infty$. First put

$$
Y^{m}:=\operatorname{span}\left\{\psi_{j} \mid j \leq m\right\} \subset W_{0, \sigma}^{1,2}\left(\mathbb{R}^{3}\right) .
$$

Since $Y^{m}$ is a finite dimensional vector space, all norms on $Y^{m}$ are equivalent. From (6.14) and (6.28) we thus deduce that $\left\{U_{n}^{m}\right\}_{n=1}^{\infty}$ is bounded in the space $L^{2}\left(\mathbb{T} ; Y^{m}\right)$ and $\left\{\mathcal{P}_{\perp} U_{n}^{m}\right\}_{n=1}^{\infty}$ in $W^{\gamma, 2}\left(\mathbb{T} ; Y^{m}\right)$ for all $\gamma \in\left[0, \frac{1}{3}\right)$. Consequently, there is a $U^{m} \in L^{2}\left(\mathbb{T} ; Y^{m}\right)$ with $\mathcal{P}_{\perp} U^{m} \in W^{\gamma, 2}\left(\mathbb{T} ; Y^{m}\right)$ and a subsequence of $\left\{U_{n}^{m}\right\}_{n=1}^{\infty}$, which we for simplicity still denote by $\left\{U_{n}^{m}\right\}_{n=1}^{\infty}$, such that

$$
\begin{aligned}
& U_{n}^{m} \rightarrow U^{m} \text { in } L^{2}\left(\mathbb{T} ; Y^{m}\right) \text { as } n \rightarrow \infty, \\
\forall \gamma \in\left[0, \frac{1}{3}\right): & \mathcal{P}_{\perp} U_{n}^{m} \rightarrow \mathcal{P}_{\perp} U^{m} \text { in } W^{\gamma, 2}\left(\mathbb{T} ; Y^{m}\right) \text { as } n \rightarrow \infty .
\end{aligned}
$$


It follows from (6.37) that $\mathcal{P} U_{n}^{m} \rightarrow \mathcal{P} U^{m}$ in $Y^{m}$, which, since $Y^{m}$ is finite dimensional, implies strong convergence $\mathcal{P} U_{n}^{m} \rightarrow \mathcal{P} U^{m}$ in $Y^{m}$ as $n \rightarrow \infty$. Moreover, again due to the fact that $Y^{m}$ is finite dimensional, $W^{\gamma_{1}, 2}\left(\mathbb{T} ; Y^{m}\right)$ is compactly embedded in $W^{\gamma_{2}, 2}\left(\mathbb{T} ; Y^{m}\right)$ for $\gamma_{1}>\gamma_{2} \geq 0$. Consequently, (6.38) implies strong convergence $\mathcal{P}_{\perp} U_{n}^{m} \rightarrow \mathcal{P}_{\perp} U^{m}$ in $W^{\gamma, 2}\left(\mathbb{T} ; Y^{m}\right)$ as $n \rightarrow \infty$ for every $\gamma \in[0,1 / 3)$. We thus have

$$
\forall \gamma \in\left[0, \frac{1}{3}\right): \quad U_{n}^{m} \rightarrow U^{m} \text { in } W^{\gamma, 2}\left(\mathbb{T} ; Y^{m}\right) \text { as } n \rightarrow \infty .
$$

Finally, in view of (6.14) and (6.28), we have

$$
\forall \gamma \in\left[0, \frac{1}{3}\right): \quad\left\|U^{m}\right\|_{L^{2}\left(\mathbb{T} ; D_{0, \sigma}^{1,2}\left(\mathbb{R}^{3}\right)\right)}+\left\|\mathcal{P}_{\perp} U^{m}\right\|_{W^{\gamma, 2}\left(\mathbb{T} ; L^{2}\left(\mathbb{R}^{3}\right)^{3}\right)} \leq c_{12}
$$

with $c_{12}$ independent on $m$. We shall now pass to the limit $n \rightarrow \infty$ in (6.36). We start by verifying that

$$
\lim _{n \rightarrow \infty} \sum_{\substack{|k|,|j| \leq n \\ j \neq 0,|j-j| \leq n}} \int_{\mathbb{R}^{3}}\left(u_{h-j-k}^{n} \cdot \nabla u_{k}^{n}\right) \cdot u_{j}^{n} \mathrm{~d} x=\int_{\mathbb{T}}\left(\int_{\mathbb{R}^{3}} U^{m} \cdot \nabla U^{m} \cdot \mathcal{P}_{\perp} U^{m} \mathrm{~d} x\right) \mathrm{e}^{-i \frac{2 \pi}{\mathcal{T}} h t} \mathrm{~d} t .
$$

Observe that the right-hand side of $(6.41)$ is well-defined since $W^{\frac{1}{4}, 2}\left(\mathbb{T} ; Y^{m}\right)$ is embedded in $L^{4}\left(\mathbb{T} ; Y^{m}\right)$ and thus $U^{m}, \nabla U^{m} \in L^{4}\left(\mathbb{T} ; Y^{m}\right)$. In fact, letting $\left\{u_{k}\right\}_{k \in \mathbb{Z}} \subset Y^{m}$ denote the Fourier coefficients of $U^{m}$ we have the identity

$$
\int_{\mathbb{T}}\left(\int_{\mathbb{R}^{3}} U^{m} \cdot \nabla U^{m} \cdot \mathcal{P}_{\perp} U^{m} \mathrm{~d} x\right) \mathrm{e}^{-i \frac{2 \pi}{T} h t} \mathrm{~d} t=\sum_{\substack{k, j \in \mathbb{Z} \\ j \neq 0}} \int_{\mathbb{R}^{3}}\left(u_{h-j-k} \cdot \nabla u_{k}\right) \cdot u_{j} \mathrm{~d} x .
$$

We further observe, recalling that $u_{k}^{n}=0$ for $|k|>n$, that

$$
\begin{aligned}
\sum_{\substack{|k|,|j| \leq n \\
j \neq 0,|h-j| \leq n}} \int_{\mathbb{R}^{3}}\left(u_{h-j-k}^{n} \cdot \nabla u_{k}^{n}\right) \cdot u_{j}^{n} \mathrm{~d} x & \\
= & \sum_{\substack{k, j \in \mathbb{Z} \\
j \neq 0,|h-j| \leq n}} \int_{\mathbb{R}^{3}}\left(u_{h-j-k}^{n} \cdot \nabla u_{k}^{n}\right) \cdot u_{j}^{n} \mathrm{~d} x \\
= & \sum_{\substack{j \in \mathbb{Z} \\
j \neq 0}} \int_{\mathbb{R}^{3}}\left(\sum_{k \in \mathbb{Z}} u_{h-j-k}^{n} \cdot \nabla u_{k}^{n}\right) \cdot\left(u_{j}^{n} \chi_{h}^{n}(j)\right) \mathrm{d} x
\end{aligned}
$$

with

$$
\chi_{h}^{n}(j):= \begin{cases}1 & \text { if }|h-j| \leq n \\ 0 & \text { if }|h-j|>n\end{cases}
$$




\section{Weak solutions}

We can thus estimate

$$
\begin{aligned}
& \left|\sum_{\substack{|k|,|j| \leq n \\
j \neq 0,|h-j| \leq n}} \int_{\mathbb{R}^{3}}\left(u_{h-j-k}^{n} \cdot \nabla u_{k}^{n}\right) \cdot u_{j}^{n} \mathrm{~d} x-\int_{\mathbb{T}}\left(\int_{\mathbb{R}^{3}} U^{m} \cdot \nabla U^{m} \cdot \mathcal{P}_{\perp} U^{m} \mathrm{~d} x\right) \mathrm{e}^{-i \frac{2 \pi}{\mathcal{T}} h t} \mathrm{~d} t\right| \\
& \leq\left|\sum_{\substack{j \in \mathbb{Z} \\
j \neq 0}} \int_{\mathbb{R}^{3}}\left(\sum_{k \in \mathbb{Z}}\left[u_{h-j-k}^{n}-u_{h-j-k}\right] \cdot \nabla u_{k}^{n}\right) \cdot\left(u_{j}^{n} \chi_{h}^{n}(j)\right) \mathrm{d} x\right| \\
& \quad+\left|\sum_{\substack{j \in \mathbb{Z} \\
j \neq 0}} \int_{\mathbb{R}^{3}}\left(\sum_{k \in \mathbb{Z}} u_{h-j-k} \cdot \nabla\left[u_{k}^{n}-u_{k}\right]\right) \cdot\left(u_{j}^{n} \chi_{h}^{n}(j)\right) \mathrm{d} x\right| \\
& \quad+\left|\sum_{\substack{j \in \mathbb{Z} \\
j \neq 0}} \int_{\mathbb{R}^{3}}\left(\sum_{k \in \mathbb{Z}} u_{h-j-k} \cdot \nabla u_{k}\right) \cdot\left(u_{j}^{n} \chi_{h}^{n}(j)-u_{j}\right) \mathrm{d} x\right| \\
& =: S_{1}+S_{2}+S_{3} .
\end{aligned}
$$

In order to estimate $S_{1}$, we utilize the embedding of $W^{\frac{1}{4}, 2}\left(\mathbb{T} ; Y^{m}\right)$ into $L^{4}\left(\mathbb{T} ; Y^{m}\right)$ in combination with (6.39) to conclude that $U_{n}^{m} \rightarrow U^{m}$ in $L^{4}\left(\mathbb{T}, Y^{m}\right)$ as $n \rightarrow \infty$. Moreover, we recall that

$$
\mathcal{F}_{\mathbb{T}}\left(\left[U_{n}^{m}(x, \cdot)-U^{m}(x, \cdot)\right] \cdot \nabla U_{n}^{m}(x, \cdot)\right)(j)=\sum_{k \in \mathbb{Z}}\left[u_{j-k}^{n}(x)-u_{j-k}(x)\right] \cdot \nabla u_{k}^{n}(x) .
$$

Employing also Plancherel's identity, we deduce

$$
\begin{aligned}
S_{1} & \leq c_{13}\left\|\sum_{k \in \mathbb{Z}}\left[u_{h-j-k}^{n}-u_{h-j-k}\right] \cdot \nabla u_{k}^{n}\right\|_{\ell^{2}\left(Y^{m}\right)}\left\|u_{j}^{n}\right\|_{\ell^{2}\left(Y^{m}\right)} \\
& =c_{14}\left\|\left[U_{n}^{m}-U^{m}\right] \cdot \nabla U_{n}^{m}\right\|_{L^{2}\left(\mathbb{T} ; Y^{m}\right)}\left\|U_{n}^{m}\right\|_{L^{2}\left(\mathbb{T} ; Y^{m}\right)} \\
& \leq c_{15}\left\|U_{n}^{m}-U^{m}\right\|_{L^{4}\left(\mathbb{T} ; Y^{m}\right)}\left\|U_{n}^{m}\right\|_{L^{4}\left(\mathbb{T} ; Y^{m}\right)}\left\|U_{n}^{m}\right\|_{L^{2}\left(\mathbb{T} ; Y^{m}\right)} \rightarrow 0 \quad \text { as } n \rightarrow \infty,
\end{aligned}
$$

where we in the last inequality once more exploit that all norms on $Y^{m}$ are equivalent. In a similar manner, we verify that $S_{2} \rightarrow 0$ as $n \rightarrow \infty$. To estimate $S_{3}$, we proceed as above and obtain

$$
\begin{aligned}
S_{3} \leq & c_{16}\left\|U^{m}\right\|_{L^{4}\left(\mathbb{T} ; Y^{m}\right)}^{2}\left\|u_{j}^{n} \chi_{h}^{n}(j)-u_{j}\right\|_{\ell^{2}\left(Y^{m}\right)} \\
\leq & c_{16}\left\|U^{m}\right\|_{L^{4}\left(\mathbb{T} ; Y^{m}\right)}^{2}\left(\left\|u_{j}^{n}-u_{j}\right\|_{\ell^{2}\left(Y^{m}\right)}+\left\|u_{j}\left(1-\chi_{h}^{n}(j)\right)\right\|_{\ell^{2}\left(Y^{m}\right)}\right) \\
= & c_{17}\left\|U^{m}\right\|_{L^{4}\left(\mathbb{T} ; Y^{m}\right)}^{2}\left(\left\|U_{n}^{m}-U^{m}\right\|_{L^{2}\left(\mathbb{T} ; Y^{m}\right)}+\left\|u_{j}\left(1-\chi_{h}^{n}(j)\right)\right\|_{\ell^{2}\left(Y^{m}\right)}\right) \\
& \rightarrow 0 \text { as } n \rightarrow \infty .
\end{aligned}
$$

We conclude (6.41). We are now able to pass to the limit $n \rightarrow \infty$ in (6.36). The limit of the sum on the right-hand side in (6.36) is computed in (6.41). Employing (6.39) to 
pass to the limit $n \rightarrow \infty$ in the other terms, we conclude that

$$
\begin{aligned}
& \int_{\mathbb{T}}\left(-i \frac{2 \pi}{\mathcal{T}} h\right)\left(\frac{1}{2} \int_{\mathbb{R}^{3}}\left|\mathcal{P}_{\perp} U^{m}(x, t)\right|^{2} \mathrm{~d} x\right) \mathrm{e}^{-i \frac{2 \pi}{\mathcal{T}} h t} \mathrm{~d} t \\
&=-\int_{\mathbb{T}}\left(\int_{\mathbb{R}^{3}} \nabla U^{m}: \nabla \mathcal{P}_{\perp} U^{m} \mathrm{~d} x+\lambda \partial_{1} U^{m} \cdot \mathcal{P}_{\perp} U^{m} \mathrm{~d} x\right) \mathrm{e}^{-i \frac{2 \pi}{\mathcal{T}}} h t \mathrm{~d} t \\
&-\int_{\mathbb{T}}\left(\int_{\mathbb{R}^{3}} U^{m} \cdot \nabla U^{m} \cdot \mathcal{P}_{\perp} U^{m} \mathrm{~d} x\right) \mathrm{e}^{-i \frac{2 \pi}{\mathcal{T}} h t} \mathrm{~d} t+\left\langle f_{h}, \mathcal{P}_{\perp} U^{m}\right\rangle .
\end{aligned}
$$

Since

$$
\begin{aligned}
& \int_{\mathbb{R}^{3}}\left(U^{m} \cdot \nabla U^{m}\right) \cdot \mathcal{P}_{\perp} U^{m} \mathrm{~d} x \\
& \quad=\int_{\mathbb{R}^{3}}\left(\mathcal{P} U^{m} \cdot \nabla \mathcal{P} U^{m}\right) \cdot \mathcal{P}_{\perp} U^{m} \mathrm{~d} x+\int_{\mathbb{R}^{3}}\left(\mathcal{P}_{\perp} U^{m} \cdot \nabla \mathcal{P} U^{m}\right) \cdot \mathcal{P}_{\perp} U^{m} \mathrm{~d} x,
\end{aligned}
$$

we obtain from (6.42) that

$$
\begin{aligned}
\left(i \frac{2 \pi}{\mathcal{T}} h\right) \int_{\mathbb{T}} \frac{1}{2}\left(\int_{\mathbb{R}^{3}}\left|\mathcal{P}_{\perp} U^{m}\right|^{2} \mathrm{~d} x\right) \mathrm{e}^{i \frac{2 \pi}{\mathcal{T}} h t} \mathrm{~d} t & =\int_{\mathbb{T}} \int_{\mathbb{R}^{3}} \nabla U^{m}: \nabla \mathcal{P}_{\perp} U^{m} \mathrm{~d} x \mathrm{e}^{i \frac{2 \pi}{\mathcal{T}} h t} \mathrm{~d} t \\
& -\lambda \iint_{\mathbb{T}} \int_{\mathbb{R}^{3}} \partial_{1} U^{m} \cdot \mathcal{P}_{\perp} U^{m} \mathrm{~d} x \mathrm{e}^{i \frac{2 \pi}{\mathcal{T}} h t} \mathrm{~d} t \\
& +\int_{\mathbb{T}} \int_{\mathbb{R}^{3}}\left(\mathcal{P} U^{m} \cdot \nabla \mathcal{P} U^{m}\right) \cdot \mathcal{P}_{\perp} U^{m} \mathrm{~d} x \mathrm{e}^{i \frac{2 \pi}{\mathcal{T}} h t} \mathrm{~d} t \\
& +\int_{\mathbb{T}} \int_{\mathbb{R}^{3}}\left(\mathcal{P}_{\perp} U^{m} \cdot \nabla \mathcal{P} U^{m}\right) \cdot \mathcal{P}_{\perp} U^{m} \mathrm{~d} x \mathrm{e}^{i \frac{2 \pi}{\mathcal{T}} h t} \mathrm{~d} t \\
& -\int_{\mathbb{T}}\left\langle f, \mathcal{P}_{\perp} U^{m}\right\rangle \mathrm{e}^{i \frac{2 \pi}{\mathcal{T}} h t} \mathrm{~d} t
\end{aligned}
$$

for any $h \in \mathbb{Z}$. Now put

$$
G(t):=\frac{1}{2} \int_{\mathbb{R}^{3}}\left|\mathcal{P}_{\perp} U^{m}(x, t)\right|^{2} \mathrm{~d} x
$$

and

$$
\begin{aligned}
H(t):= & \int_{\mathbb{R}^{3}} \nabla U^{m}: \nabla \mathcal{P}_{\perp} U^{m} \mathrm{~d} x-\lambda \int_{\mathbb{R}^{3}} \partial_{1} U^{m} \cdot \mathcal{P}_{\perp} U^{m} \mathrm{~d} x+\int_{\mathbb{R}^{3}}\left(\mathcal{P} U^{m} \cdot \nabla \mathcal{P} U^{m}\right) \cdot \mathcal{P}_{\perp} U^{m} \mathrm{~d} x \\
& +\int_{\mathbb{R}^{3}}\left(\mathcal{P}_{\perp} U^{m} \cdot \nabla \mathcal{P} U^{m}\right) \cdot \mathcal{P}_{\perp} U^{m} \mathrm{~d} x-\left\langle f, \mathcal{P}_{\perp} U^{m}\right\rangle .
\end{aligned}
$$




\section{Weak solutions}

From (6.40) we already know that $G \in L^{1}(\mathbb{T})$ with $\|G\|_{L^{1}(\mathbb{T})} \leq c_{12}{ }^{2}$. From (6.43) we see that $H$ is the distributional derivative of $G$. We shall verify that $H \in L^{1}(\mathbb{T})$ and estimate $\|H\|_{L^{1}(\mathbb{T})}$. For this purpose, we estimate, recalling (6.40),

$$
\int_{\mathbb{T}}\left|\int_{\mathbb{R}^{3}} \nabla U^{m}: \nabla \mathcal{P}_{\perp} U^{m} \mathrm{~d} x\right| \mathrm{d} t \leq\left\|U^{m}\right\|_{L^{2}\left(\mathbb{T} ; D_{0, \sigma}^{1,2}\left(\mathbb{R}^{3}\right)\right)}^{2} \leq c_{12}{ }^{2}
$$

and

$$
\int_{\mathbb{T}}\left|\int_{\mathbb{R}^{3}} \partial_{1} U^{m} \cdot \mathcal{P}_{\perp} U^{m} \mathrm{~d} x\right| \mathrm{d} t \leq\left\|U^{m}\right\|_{L^{2}\left(\mathbb{T} ; D_{0, \sigma}^{1,2}\left(\mathbb{R}^{3}\right)\right)}\left\|\mathcal{P}_{\perp} U^{m}\right\|_{L^{2}\left(\mathbb{T} ; L^{2}\left(\mathbb{R}^{3}\right)\right)} \leq c_{12}{ }^{2} .
$$

Employing Lemma 2.3.1, (2.2), and Hölder's inequality, we find, recalling again (6.40), that

$$
\begin{aligned}
\int_{\mathbb{T}}\left|\int_{\mathbb{R}^{3}}\left(\mathcal{P} U^{m} \cdot \nabla \mathcal{P} U^{m}\right) \cdot \mathcal{P}_{\perp} U^{m} \mathrm{~d} x\right| \mathrm{d} t \\
\leq \int_{\mathbb{T}}\left\|\mathcal{P} U^{m}\right\|_{6}\left\|\nabla \mathcal{P} U^{m}\right\|_{2}\left\|\mathcal{P}_{\perp} U^{m}(t)\right\|_{3} \mathrm{~d} t \\
\leq\left\|\nabla \mathcal{P} U^{m}\right\|_{2}^{2} \int_{\mathbb{T}}\left\|\nabla \mathcal{P}_{\perp} U^{m}(t)\right\|_{2}^{\frac{1}{2}}\left\|\mathcal{P}_{\perp} U^{m}(t)\right\|_{2}^{\frac{1}{2}} \mathrm{~d} t \\
\leq\left\|\nabla \mathcal{P} U^{m}\right\|_{2}^{2}\left\|\mathcal{P}_{\perp} U^{m}\right\|_{L^{2}\left(\mathbb{T} ; D_{0, \sigma}^{1,2}\left(\mathbb{R}^{3}\right)\right)}^{\frac{1}{2}}\left\|\mathcal{P}_{\perp} U^{m}\right\|_{L^{2}\left(\mathbb{T} ; L^{2}\left(\mathbb{R}^{3}\right)\right)}^{\frac{1}{2}} \\
\leq c_{12}{ }^{3} .
\end{aligned}
$$

Similarly, we estimate

$$
\begin{aligned}
\int_{\mathbb{T}} \mid \int_{\mathbb{R}^{3}}\left(\mathcal{P}_{\perp} U^{m} \cdot \nabla \mathcal{P}\right. & \left.U^{m}\right) \cdot \mathcal{P}_{\perp} U^{m} \mathrm{~d} x \mid \mathrm{d} t \\
& \leq\left\|\nabla \mathcal{P} U^{m}\right\|_{2} \int_{\mathbb{T}}\left\|\mathcal{P}_{\perp} U^{m}(t)\right\|_{4}^{2} \mathrm{~d} t \\
& \leq\left\|\nabla \mathcal{P} U^{m}\right\|_{2} \int_{\mathbb{T}}\left\|\mathcal{P}_{\perp} U^{m}(t)\right\|_{2}^{\frac{1}{2}}\left\|\nabla \mathcal{P}_{\perp} U^{m}(t)\right\|_{2}^{\frac{3}{2}} \mathrm{~d} t \\
& \leq\left\|\nabla \mathcal{P} U^{m}\right\|_{2}\left\|\mathcal{P}_{\perp} U^{m}\right\|_{L^{2}\left(\mathbb{T} ; L^{2}\left(\mathbb{R}^{3}\right)\right)}^{\frac{1}{2}}\left\|\mathcal{P}_{\perp} U^{m}\right\|_{L^{2}\left(\mathbb{T} ; D_{0, \sigma}^{1,2}\left(\mathbb{R}^{3}\right)\right)}^{\frac{3}{2}} \\
& \leq c_{12}{ }^{3} .
\end{aligned}
$$

Finally, we observe that

$$
\begin{aligned}
\int_{\mathbb{T}}\left|\left\langle f, \mathcal{P}_{\perp} U^{m}\right\rangle\right| \mathrm{d} t & \leq\|f\|_{L^{2}\left(\mathbb{T} ; D_{0}^{-1,2}\left(\mathbb{R}^{3}\right)\right)}\left\|\mathcal{P}_{\perp} U^{m}\right\|_{L^{2}\left(\mathbb{T} ; D_{0, \sigma}^{1,2}\left(\mathbb{R}^{3}\right)\right)} \\
& \leq c_{12}\|f\|_{L^{2}\left(\mathbb{T} ; D_{0}^{-1,2}\left(\mathbb{R}^{3}\right)\right)}
\end{aligned}
$$


By (6.44)-(6.48), it follows that $H \in L^{1}(\mathbb{T})$. We thus deduce $G \in W^{1,1}(\mathbb{T})$ with

$$
\|G\|_{W^{1,1}(\mathbb{T})} \leq c_{12}^{2}+\lambda c_{12}^{2}+c_{12}^{3}+c_{12}\|f\|_{L^{2}\left(\mathbb{T} ; D_{0}^{-1,2}\left(\mathbb{R}^{3}\right)\right)} .
$$

Since $W^{1,1}(\mathbb{T})$ is continuously embedded in $L^{\infty}(\mathbb{T})$, we conclude that

$$
\left\|\mathcal{P}_{\perp} U^{m}\right\|_{L^{\infty}\left(\mathbb{T} ; L^{2}\left(\mathbb{R}^{3}\right)\right)}=4\|G\|_{L^{\infty}(\mathbb{T})}^{\frac{1}{2}} \leq c_{18}
$$

with $c_{18}=c_{18}(\lambda,\|f\|)$ independent on $m$.

We now verify that $U^{m}$ is an approximate solution to (6.1). For any $\Phi \in X_{n_{0}}^{m}$ we have

$$
\int_{\mathbb{T}} \int_{\mathbb{R}^{3}}-U_{n}^{m} \cdot \partial_{t} \Phi+\nabla U_{n}^{m}: \nabla \Phi-\lambda \partial_{1} U_{n}^{m} \cdot \Phi+\left(U_{n}^{m} \cdot \nabla U_{n}^{m}\right) \cdot \Phi \mathrm{d} x \mathrm{~d} t=\int_{\mathbb{T}}\langle f, \Phi\rangle \mathrm{d} t
$$

for $n \geq n_{0}$. We can pass to the limit $n \rightarrow \infty$ in (6.50) in the same manner we derived (6.43) by passing to the limit $n \rightarrow \infty$ in (6.36). In particular, we can utilize (6.39) to show

$$
\begin{aligned}
\forall \Phi \in & \bigcup_{n_{0} \in \mathbb{N}} X_{n_{0}}^{m}: \\
& \int_{\mathbb{T}} \int_{\mathbb{R}^{3}}-U^{m} \cdot \partial_{t} \Phi+\nabla U^{m}: \nabla \Phi-\lambda \partial_{1} U^{m} \cdot \Phi+\left(U^{m} \cdot \nabla U^{m}\right) \cdot \Phi \mathrm{d} x \mathrm{~d} t=\int_{\mathbb{T}}\langle f, \Phi\rangle \mathrm{d} t .
\end{aligned}
$$

We summarize at this point that $U^{m} \in L^{2}\left(\mathbb{T} ; D_{0, \sigma}^{1,2}\left(\mathbb{R}^{3}\right)\right)$ is an approximate solution to (6.1) that satisfies (6.40) and (6.49).

To obtain a proper weak solution to (6.1), we let $m \rightarrow \infty$. By (6.40), the sequence $\left\{U^{m}\right\}_{m=1}^{\infty}$ is bounded in $L^{2}\left(\mathbb{T} ; D_{0, \sigma}^{1,2}\left(\mathbb{R}^{3}\right)\right)$. Thus, there is a $U \in L^{2}\left(\mathbb{T} ; D_{0, \sigma}^{1,2}\left(\mathbb{R}^{3}\right)\right)$ and a subsequence of $\left\{U^{m}\right\}_{m=1}^{\infty}$, which we for simplicity still denote by $\left\{U^{m}\right\}_{m=1}^{\infty}$, that converges weakly towards $U$ as $m \rightarrow \infty$. In view of (6.40) and (6.49), we see that

$$
\gamma \in\left[0, \frac{1}{3}\right): \quad \mathcal{P}_{\perp} U \in W^{\gamma, 2}\left(\mathbb{T} ; L^{2}\left(\mathbb{R}^{3}\right)^{3}\right) \cap L^{\infty}\left(\mathbb{T} ; L^{2}\left(\mathbb{R}^{3}\right)^{3}\right),
$$

and we can pick any $\gamma \in\left(0, \frac{1}{3}\right)$ and choose the subsequence in such a way that

$$
\begin{array}{ll}
U^{m} \rightarrow U & \text { in } L^{2}\left(\mathbb{T} ; D_{0, \sigma}^{1,2}\left(\mathbb{R}^{3}\right)\right), \\
\mathcal{P}_{\perp} U^{m} \rightarrow \mathcal{P}_{\perp} U & \text { in } W^{\gamma, 2}\left(\mathbb{T} ; L^{2}\left(\mathbb{R}^{3}\right)^{3}\right), \\
\mathcal{P}_{\perp} U^{m} \rightarrow^{*} \mathcal{P}_{\perp} U & \text { in } \quad L^{\infty}\left(\mathbb{T} ; L^{2}\left(\mathbb{R}^{3}\right)^{3}\right), \quad \text { as } m \rightarrow \infty,
\end{array}
$$

where $\rightarrow^{*}$ denotes convergence with respect to the weak*-topology of $L^{\infty}\left(\mathbb{T} ; L^{2}\left(\mathbb{R}^{3}\right)^{3}\right)$. For $K \subset \mathbb{R}^{3}$ a compact set, $W^{\gamma, 2}\left(\mathbb{T} ; L^{2}\left(\mathbb{R}^{3}\right)^{3}\right) \cap L^{2}\left(\mathbb{T} ; D_{0}^{1,2}\left(\mathbb{R}^{3}\right)^{3}\right)$ is compactly embedded 


\section{Weak solutions}

in $L^{2}\left(\mathbb{T} ; L^{2}(K)^{3}\right)$; see for example [56, Chapter II, $\S 2$, Theorem 2.2]. Moreover, $D_{0, \sigma}^{1,2}\left(\mathbb{R}^{3}\right)$ is compactly embedded in $L^{2}(K)^{3}$. We therefore also have

$$
\begin{array}{lll}
\forall K \subset \mathbb{R}^{3}, K \text { compact : } & \mathcal{P}_{\perp} U^{m} \rightarrow \mathcal{P}_{\perp} U & \text { in } L^{2}\left(\mathbb{T} ; L^{2}(K)^{3}\right), \\
\forall K \subset \mathbb{R}^{3}, K \text { compact : } & \mathcal{P} U^{m} \rightarrow \mathcal{P} U & \text { in } L^{2}(K)^{3} .
\end{array}
$$

By virtue of (6.51) and (6.53)-(6.54), it can now be shown that $U$ is a weak solution to (6.1). Consider first $\Phi \in X_{n_{0}}^{m_{0}}$ for some $n_{0}, m_{0} \in \mathbb{N}$. With this test function fixed, we pass to the limit $m \rightarrow \infty$ in (6.51). It is easy to pass to the limit in the terms that are linear in $U^{m}$. To verify that we can also pass to the limit in the nonlinear term, we first compute

$$
\begin{aligned}
\int_{\mathbb{T}} \int_{\mathbb{R}^{3}}\left(U^{m} \cdot \nabla U^{m}\right) \cdot \Phi \mathrm{d} x \mathrm{~d} t= & \int_{\mathbb{T}} \int_{\mathbb{R}^{3}}\left(\mathcal{P} U^{m} \cdot \nabla \Phi\right) \cdot \mathcal{P} U^{m} \mathrm{~d} x \mathrm{~d} t \\
& +\int_{\mathbb{T}} \int_{\mathbb{R}^{3}}\left(\mathcal{P} U^{m} \cdot \nabla \Phi\right) \cdot \mathcal{P}_{\perp} U^{m} \mathrm{~d} x \mathrm{~d} t \\
& +\int_{\mathbb{T}} \int_{\mathbb{R}^{3}}\left(\mathcal{P}_{\perp} U^{m} \cdot \nabla \Phi\right) \cdot \mathcal{P} U^{m} \mathrm{~d} x \mathrm{~d} t \\
& +\int_{\mathbb{T}} \int_{\mathbb{R}^{3}}\left(\mathcal{P}_{\perp} U^{m} \cdot \nabla \Phi\right) \cdot \mathcal{P}_{\perp} U^{m} \mathrm{~d} x \mathrm{~d} t .
\end{aligned}
$$

We can estimate

$$
\begin{aligned}
\left|\int_{\mathbb{T}} \int_{\mathbb{R}^{3}}\left(\mathcal{P} U^{m} \cdot \nabla \Phi\right) \cdot \mathcal{P} U^{m} \mathrm{~d} x \mathrm{~d} t-\int_{\mathbb{T}} \int_{\mathbb{R}^{3}}(\mathcal{P} U \cdot \nabla \Phi) \cdot \mathcal{P} U \mathrm{~d} x \mathrm{~d} t\right| \\
\leq c_{19}\left\|\mathcal{P} U^{m}-\mathcal{P} U\right\|_{L^{2}(K)}\left(\|\mathcal{P} U\|_{L^{2}(K)}+\left\|\mathcal{P} U^{m}\right\|_{L^{2}(K)}\right)
\end{aligned}
$$

with $K:=\bigcup_{j \leq m_{0}} \operatorname{supp} \psi_{j}$, and $c_{19}$ independent on $m$. Similarly, we estimate

$$
\begin{array}{r}
\left|\int_{\mathbb{T}} \int_{\mathbb{R}^{3}}\left(\mathcal{P} U^{m} \cdot \nabla \Phi\right) \cdot \mathcal{P}_{\perp} U^{m} \mathrm{~d} x \mathrm{~d} t-\int_{\mathbb{T}} \int_{\mathbb{R}^{3}}(\mathcal{P} U \cdot \nabla \Phi) \cdot \mathcal{P}_{\perp} U \mathrm{~d} x \mathrm{~d} t\right| \\
\leq c_{20}\left(\left\|\mathcal{P} U^{m}-\mathcal{P} U\right\|_{L^{2}(K)}\left\|\mathcal{P}_{\perp} U^{m}\right\|_{L^{2}\left(\mathbb{T} ; L^{2}(K)\right)}\right. \\
\left.+\|\mathcal{P} U\|_{L^{2}(K)}\left\|\mathcal{P}_{\perp} U^{m}-\mathcal{P}_{\perp} U\right\|_{L^{2}\left(\mathbb{T} ; L^{2}(K)\right)}\right),
\end{array}
$$

and

$$
\begin{gathered}
\left|\int_{\mathbb{T}} \int_{\mathbb{R}^{3}}\left(\mathcal{P}_{\perp} U^{m} \cdot \nabla \Phi\right) \cdot \mathcal{P} U^{m} \mathrm{~d} x \mathrm{~d} t-\int_{\mathbb{T}} \int_{\mathbb{R}^{3}}\left(\mathcal{P}_{\perp} U \cdot \nabla \Phi\right) \cdot \mathcal{P} U \mathrm{~d} x \mathrm{~d} t\right| \\
\leq c_{21}\left(\left\|\mathcal{P} U^{m}-\mathcal{P} U\right\|_{L^{2}(K)}\left\|\mathcal{P}_{\perp} U^{m}\right\|_{L^{2}\left(\mathbb{T} ; L^{2}(K)\right)}\right. \\
\left.\quad+\|\mathcal{P} U\|_{L^{2}(K)}\left\|\mathcal{P}_{\perp} U^{m}-\mathcal{P}_{\perp} U\right\|_{L^{2}\left(\mathbb{T} ; L^{2}(K)\right)}\right) .
\end{gathered}
$$


Finally, we also have

$$
\begin{aligned}
& \left|\int_{\mathbb{T}} \int_{\mathbb{R}^{3}}\left(\mathcal{P}_{\perp} U^{m} \cdot \nabla \Phi\right) \cdot \mathcal{P}_{\perp} U^{m} \mathrm{~d} x \mathrm{~d} t-\int_{\mathbb{T}} \int_{\mathbb{R}^{3}}\left(\mathcal{P}_{\perp} U \cdot \nabla \Phi\right) \cdot \mathcal{P}_{\perp} U \mathrm{~d} x \mathrm{~d} t\right| \\
& \leq c_{22}\left\|\mathcal{P}_{\perp} U^{m}-\mathcal{P} U\right\|_{L^{2}\left(\mathbb{T} ; L^{2}(K)\right)}\left(\left\|\mathcal{P}_{\perp} U\right\|_{L^{2}\left(\mathbb{T} ; L^{2}(K)\right)}+\left\|\mathcal{P}_{\perp} U^{m}\right\|_{L^{2}\left(\mathbb{T} ; L^{2}(K)\right)} .\right.
\end{aligned}
$$

Clearly, $K \subset \mathbb{R}^{3}$ is compact. Thus, the estimates above together with (6.54) imply that we can pass to the limit $m \rightarrow \infty$ in (6.55) to obtain

$$
\lim _{m \rightarrow \infty} \int_{\mathbb{T}} \int_{\mathbb{R}^{3}}\left(U^{m} \cdot \nabla U^{m}\right) \cdot \Phi \mathrm{d} x \mathrm{~d} t=\int_{\mathbb{T}} \int_{\mathbb{R}^{3}}(U \cdot \nabla U) \cdot \Phi \mathrm{d} x \mathrm{~d} t .
$$

Returning to (6.51), we find after letting $m \rightarrow \infty$ that

$$
\begin{aligned}
\forall \Phi \in & \bigcup_{n_{0}, m_{0} \in \mathbb{N}} X_{n_{0}}^{m_{0}}: \\
& \int_{\mathbb{T}} \int_{\mathbb{R}^{3}}-U \cdot \partial_{t} \Phi+\nabla U: \nabla \Phi-\lambda \partial_{1} U \cdot \Phi+(U \cdot \nabla U) \cdot \Phi \mathrm{d} x \mathrm{~d} t=\int_{\mathbb{T}}\langle f, \Phi\rangle \mathrm{d} t .
\end{aligned}
$$

To finalize to proof, we need to extend the identity above to all $\Phi \in C_{0, \sigma}^{\infty}\left(\mathbb{R}^{3} \times \mathbb{T}\right)$. For this purpose, we put

$$
\Phi_{j, k}(x, t):=\left(1+\left(\frac{2 \pi}{\mathcal{T}}\right)^{2}|k|^{2}\right)^{-\frac{1}{2}} \mathrm{e}^{i \frac{2 \pi}{\mathcal{T}} k t} \psi_{j}(x)
$$

and utilize that $\left\{\Phi_{j, k} \mid k, j \in \mathbb{Z}\right\}$ is an orthonormal basis in $W^{1,2}\left(\mathbb{T} ; W_{0, \sigma}^{1,2}\left(\mathbb{R}^{3}\right)\right)$. Consider $\Phi \in C_{0, \sigma}^{\infty}\left(\mathbb{R}^{3} \times \mathbb{T}\right)$. Let $\langle\cdot, \cdot\rangle_{W^{1,2}\left(\mathbb{T} ; W^{1,2}\left(\mathbb{R}^{3}\right)^{3}\right)}$ denote the canonical inner product of $W^{1,2}\left(\mathbb{T} ; W^{1,2}\left(\mathbb{R}^{3}\right)^{3}\right)$ and define

$$
\Phi_{m}(x, t):=\sum_{|k|,|j| \leq m}\left\langle\Phi, \Phi_{j, k}\right\rangle_{W^{1,2}\left(\mathbb{T} ; W^{1,2}\left(\mathbb{R}^{3}\right)^{3}\right)} \Phi_{j, k} .
$$

We then use $\Phi_{m}$ as a test function in (6.56) and pass to the limit $m \rightarrow \infty$. Observe that

$$
\begin{aligned}
\left|\int_{\mathbb{T}} \int_{\mathbb{R}^{3}} U \cdot \partial_{t} \Phi-U \cdot \partial_{t} \Phi_{m} \mathrm{~d} x \mathrm{~d} t\right| & =\left|\int_{\mathbb{T}} \int_{\mathbb{R}^{3}} \mathcal{P}_{\perp} U \cdot \partial_{t} \Phi-\mathcal{P}_{\perp} U \cdot \partial_{t} \Phi_{m} \mathrm{~d} x \mathrm{~d} t\right| \\
& \leq\left\|\mathcal{P}_{\perp} U\right\|_{L^{2}\left(\mathbb{T} ; L^{2}\left(\mathbb{R}^{3}\right)\right)}\left\|\Phi-\Phi_{m}\right\|_{W^{1,2}\left(L^{2}\left(\mathbb{R}^{3}\right)\right)}
\end{aligned}
$$

whence

$$
\lim _{m \rightarrow \infty} \int_{\mathbb{T}} \int_{\mathbb{R}^{3}} U \cdot \partial_{t} \Phi_{m} \mathrm{~d} x \mathrm{~d} t=\iint_{\mathbb{T}} \int_{\mathbb{R}^{3}} U \cdot \partial_{t} \Phi \mathrm{d} x \mathrm{~d} t
$$




\section{Weak solutions}

We also note that

$$
\begin{aligned}
\mid \int_{\mathbb{T}} \int_{\mathbb{R}^{3}}(U \cdot \nabla U) \cdot \Phi & -(U \cdot \nabla U) \cdot \Phi_{m} \mathrm{~d} x \mathrm{~d} t \mid \\
& \leq \int_{\mathbb{T}}\|U(t)\|_{6}\|\nabla U(t)\|_{2}\left\|\Phi(t)-\Phi_{m}(t)\right\|_{3} \mathrm{~d} t \\
& \leq c_{23}\|U\|_{L^{2}\left(\mathbb{T} ; D_{0, \sigma}^{1,2}\left(\mathbb{R}^{3}\right)\right)}^{2} \sup _{t \in \mathrm{T}}\left\|\Phi-\Phi_{m}\right\|_{W_{0, \sigma}^{1,2}\left(\mathbb{R}^{3}\right)} \\
& \leq c_{24}\|U\|_{L^{2}\left(\mathbb{T} ; D_{0, \sigma}^{1,2}\left(\mathbb{R}^{3}\right)\right)}^{2}\left\|\Phi-\Phi_{m}\right\|_{W^{1,2}\left(W_{0, \sigma}^{1,2}\left(\mathbb{R}^{3}\right)\right)}
\end{aligned}
$$

which implies

$$
\lim _{m \rightarrow \infty} \int_{\mathbb{T}} \int_{\mathbb{R}^{3}}(U \cdot \nabla U) \cdot \Phi_{m} \mathrm{~d} x \mathrm{~d} t=\int_{\mathbb{T}} \int_{\mathbb{R}^{3}}(U \cdot \nabla U) \cdot \Phi \mathrm{d} x \mathrm{~d} t .
$$

It is now easy to verify, after inserting $\Phi_{m}$ as a test function in (6.56) and passing to the limit $m \rightarrow \infty$, that $U$ and $\Phi$ satisfy (6.4). Thus, recalling (6.52), we have shown that $U \in L^{2}\left(\mathbb{T} ; D_{0, \sigma}^{1,2}\left(\mathbb{R}^{3}\right)\right)$ is a weak time-periodic solution to (6.1) that satisfies (6.8)(6.10).

Remark 6.3.2. Observe that the condition $\gamma>\frac{1}{3}$ needed to conclude (6.27) is not dictated by the regularity of the data $f$. In other words, assuming more regularity on the data $f$ will not lead to any improvement in (6.10) and thereby to better regularity of the solution $u$, at least not in the proof above.

Remark 6.3.3. We shall briefly compare Theorem 6.3.1 with the similar well-known result for the the initial-value Navier-Stokes problem

$$
\begin{cases}\partial_{t} u-\Delta u-\lambda \partial_{1} u+\nabla \mathfrak{p}+u \cdot \nabla u=f & \text { in } \mathbb{R}^{3} \times(0, T), \\ \operatorname{div} u=0 & \text { in } \mathbb{R}^{3} \times(0, T), \\ u(\cdot, 0)=u_{0} & \text { in } \mathbb{R}^{3}\end{cases}
$$

Existence of a weak solution $u \in L^{2}\left(0, T ; D_{0, \sigma}^{1,2}\left(\mathbb{R}^{3}\right)\right)$ to $(6.57)$ was originally shown by LERAY in [42]. A proof based on a Galerkin approximation was given by Hopf in [32]. Compared to the proof of Theorem 6.3.1 above, which, of course, is also based on a Galerkin approximation, it is much simpler in the proof Hopf to obtain $u \in$ $L^{\infty}\left(0, T ; L^{2}\left(\mathbb{R}^{3}\right)^{3}\right)$. Indeed, multiplying $(6.57)_{1}$ with $u$ and performing for arbitrary $t \in(0, t)$ the formal integration

$$
\int_{0}^{t} \int_{\mathbb{R}^{3}} \partial_{t} u \cdot u-\Delta u \cdot u-\lambda \partial_{1} u \cdot u+\nabla \mathfrak{p} \cdot u+(u \cdot \nabla u) \cdot u \mathrm{~d} x \mathrm{~d} t=\int_{0}^{t} \int_{\mathbb{R}^{3}} f \cdot u \mathrm{~d} x \mathrm{~d} t,
$$

one finds, after a formal integration by parts and a simple estimate, that

$$
\|u(t)\|_{2} \leq\|u(0)\|_{2}+2\|f\|_{L^{2}\left(0, T ; D_{0}^{-1,2}\left(\mathbb{R}^{3}\right)\right)}\|u\|_{L^{2}\left(0, T ; D_{0, \sigma}^{1,2}\left(\mathbb{R}^{3}\right)\right)} .
$$


The computation above can only be performed formally at first, but since it is valid on the finite-dimensional level in the Galerkin approximation, it eventually leads to the validity of (6.58) also for the final weak solution $u$. Since $\|u(0)\|_{2}$ equals the prescribed quantity $u_{0},(6.58)$ implies that $\|u(t)\|_{2}$ is bounded independently on $t$ and thus $u \in$ $L^{\infty}\left(0, T ; L^{2}\left(\mathbb{R}^{3}\right)^{3}\right)$. In contrast, in the time-periodic case there is no information available on $\|u(0)\|_{2}$. Consequently, the simple argument that yields $u \in L^{\infty}\left(0, T ; L^{2}\left(\mathbb{R}^{3}\right)^{3}\right)$ for the initial-value problem cannot be used in the time-periodic case.

Remark 6.3.4. It may seem surprising at first that Theorem 6.3.1 only establishes that the projection $\mathcal{P}_{\perp} u$ lies in $L^{\infty}\left(0, \mathcal{T} ; L^{2}\left(\mathbb{R}^{3}\right)^{3}\right)$ and not $u$ itself, which, as shown above, can be proved rather easily for the corresponding initial-value problem. A short investigation, however, reveals that $u$ cannot in general belong to $L^{\infty}\left(0, \mathcal{T} ; L^{2}\left(\mathbb{R}^{3}\right)^{3}\right)$ in the timeperiodic case. If, for example, the prescribed data $f$ is time independent, the Galerkin method employed in the proof of Theorem 6.3.1 may yield a time-independent weak solution $u$, that is, a steady-state solution to

$$
\begin{cases}-\Delta u-\lambda \partial_{1} u+\nabla \mathfrak{p}+u \cdot \nabla u=f & \text { in } \mathbb{R}^{3}, \\ \operatorname{div} u=0 & \text { in } \mathbb{R}^{3} .\end{cases}
$$

Indeed, it is easy to verify that the vector $\xi_{0}=\left(\xi^{\#}, \xi^{*}, \xi^{0}\right) \in \mathbb{R}^{m \times n} \times \mathbb{R}^{m \times n} \times \mathbb{R}^{m}$ in (6.13) may be chosen such that $\xi^{\#}=\xi^{*}=0$ if $f$ is time independent. By explicitly choosing $\xi_{0}$ in this way, the existence part of the proof reduces completely to the proof of [18, Theorem IX.4.1], which yields a weak so-called Leray solution to (6.59). Provided $f$ is of compact support and possesses a certain amount of regularity, it was shown by FINN [11] in the case $\lambda \neq 0$ that such a solution does not belong to $L^{2}\left(\mathbb{R}^{3}\right)^{3}$ when $\int_{\mathbb{R}^{3}} f \mathrm{~d} x \neq 0$; see also [18, Theorem IX.7.2]. Consequently, $u \notin L^{\infty}\left(0, T ; L^{2}\left(\mathbb{R}^{3}\right)^{3}\right)$ in this case. If we further assume that $f$ is sufficiently small, we can use a recent result by KOROLEV and Šverák [35] to derive $u \notin L^{\infty}\left(0, T ; L^{2}\left(\mathbb{R}^{3}\right)^{3}\right)$ also in the case $\lambda=0$.

Remark 6.3.5. There are two important implications of the property

$$
\mathcal{P}_{\perp} u \in L^{\infty}\left(0, \mathcal{T} ; L^{2}\left(\mathbb{R}^{3}\right)^{3}\right) .
$$

In physical terms, (6.60) implies that the weak time-periodic solution $u$ can be written as a sum of a steady-state part $\mathcal{P} u$ and a time-dependent part $\mathcal{P}_{\perp} u$ with finite kinetic energy. In mathematical terms, (6.60) is important to allow for development of further regularity properties. Experience from the initial-value problem, which has been studied much more intensively over the years, shows that additional regularity for $u$ in time is the most difficult to establish. Since $u=\mathcal{P} u+\mathcal{P}_{\perp} u$ and $\mathcal{P} u$ is time-independent, $\mathcal{P}_{\perp} u \in L^{\infty}\left(0, \mathcal{T} ; L^{2}\left(\mathbb{R}^{3}\right)^{3}\right)$ is just as good a starting point for an investigation into further time regularity as $u \in L^{\infty}\left(0, \mathcal{T} ; L^{2}\left(\mathbb{R}^{3}\right)^{3}\right)$ is for the initial-value problem. In other words, (6.60) "levels the playing field" for the time-periodic and initial-value Navier-Stokes problem in this respect. 


\subsection{Regularity properties}

The purpose of this section is to show that the weak solution from Theorem 6.3.1 is continuous in time with respect an appropriate topology. Continuity in time is needed to conclude that the solution is in fact time-periodic in a classical pointwise fashion. Obviously, a statement of continuity in time concerning an element $u \in L^{2}\left(\mathbb{T} ; D_{0, \sigma}^{1,2}\left(\mathbb{R}^{3}\right)\right)$ can be made only up to a modification of the function on a set of measure zero.

From the weak formulation (6.4), time regularity of the solution in a relatively weak topology can be derived almost directly. More specifically, we have:

Lemma 6.4.1. Let $f \in L^{2}\left(\mathbb{T} ; D_{0}^{-1,2}\left(\mathbb{R}^{3}\right)^{3}\right)$ and $\lambda \geq 0$. The weak time-periodic solution $u \in L^{2}\left(\mathbb{T} ; D_{0, \sigma}^{1,2}\left(\mathbb{R}^{3}\right)\right)$ from Theorem 6.3.1 satisfies

$$
\forall N \in \mathbb{N}: \quad u \in W^{1,1}\left(\mathbb{T} ; D_{0, \sigma}^{-1,2}\left(\mathrm{~B}_{N}\right)\right)
$$

with

$$
\begin{aligned}
& \forall \varphi \in D_{0, \sigma}^{1,2}\left(\mathrm{~B}_{N}\right): \\
& -\left\langle\partial_{t} u(t), \varphi\right\rangle=\int_{\mathbb{R}^{3}} \nabla u(t): \nabla \varphi-\lambda \partial_{1} u(t) \cdot \varphi+(u(t) \cdot \nabla u(t)) \cdot \varphi-\langle f(t), \varphi\rangle \mathrm{d} x
\end{aligned}
$$

for a.e. $t \in \mathbb{T}$.

Proof. Let $N \in \mathbb{N}$. For $t \in \mathbb{T}$ we let $g(t) \in D_{0, \sigma}^{-1,2}\left(\mathrm{~B}_{N}\right)$ denote the functional

$$
\langle g(t), \varphi\rangle:=\int_{\mathbb{R}^{3}} \nabla u(t): \nabla \varphi-\lambda \partial_{1} u(t) \cdot \varphi+(u(t) \cdot \nabla u(t)) \cdot \varphi \mathrm{d} x-\langle f(t), \varphi\rangle .
$$

We can estimate

$$
\begin{aligned}
& |\langle g(t), \varphi\rangle| \\
& \quad \leq\|\nabla u(t)\|_{2}\|\nabla \varphi\|_{2}+\lambda\|\nabla u(t)\|_{2}\|\varphi\|_{2}+\left|\int_{\mathbb{R}^{3}}(u(t) \cdot \nabla \varphi) \cdot u(t) \mathrm{d} x\right|+|f(t)|_{-1,2}\|\nabla \varphi\|_{2} \\
& \quad \leq c_{1}\left(\|\nabla u(t)\|_{2}+\|u(t)\|_{L^{4}\left(\mathrm{~B}_{N}\right)}^{2}+|f(t)|_{-1,2}\right)\|\nabla \varphi\|_{2} \\
& \quad \leq c_{2}\left(\|\nabla u(t)\|_{2}+\|\nabla u(t)\|_{2}^{2}+|f(t)|_{-1,2}\right)\|\nabla \varphi\|_{2},
\end{aligned}
$$

with $c_{2}=c_{2}(N)$. It follows that $g \in L^{1}\left(\mathbb{T} ; D_{0, \sigma}^{-1,2}\left(\mathrm{~B}_{N}\right)\right)$. Since $u \in L^{2}\left(\mathbb{T} ; D_{0, \sigma}^{1,2}\left(\mathbb{R}^{3}\right)\right)$, we immediately obtain $u \in L^{1}\left(\mathbb{T} ; D_{0, \sigma}^{-1,2}\left(\mathrm{~B}_{N}\right)\right)$. To show (6.61) and (6.62), we therefore only need to verify $-\partial_{t} u=g$. For this purpose, we want to use, for arbitrary $k \in \mathbb{Z}$ and $\varphi \in D_{0, \sigma}^{1,2}\left(\mathrm{~B}_{N}\right), \Phi(x, t):=\varphi(x) \mathrm{e}^{i \frac{2 \pi}{T} k t}$ as a test function in the weak formulation (6.4). Since $\Phi(x, t)$ is not admissible as a test function, we first approximate $\varphi \in D_{0, \sigma}^{1,2}\left(\mathrm{~B}_{N}\right)$ by a sequence $\left\{\varphi_{n}\right\}_{n=1}^{\infty} \subset C_{0, \sigma}^{\infty}\left(\mathrm{B}_{N}\right)$ in the $|\cdot|_{1,2}$-norm. Using $\Phi_{n}(x, t):=\varphi_{n}(x) \mathrm{e}^{i \frac{2 \pi}{T} k t}$ as a 
test function and subsequently letting $n \rightarrow \infty$, it is easy to verify that

$$
\begin{aligned}
& i \frac{2 \pi}{\mathcal{T}} k \int_{\mathbb{T}} \int_{\mathbb{R}^{3}} u(x, t) \cdot \varphi(x) \mathrm{d} x \mathrm{e}^{i \frac{2 \pi}{\mathcal{T}} k t} \mathrm{~d} t \\
& \quad=\int_{\mathbb{T}}\left(\int_{\mathbb{R}^{3}} \nabla u: \nabla \varphi-\lambda \partial_{1} u \cdot \varphi+(u \cdot \nabla u) \cdot \varphi \mathrm{d} x\right) \mathrm{e}^{i \frac{2 \pi}{\mathcal{T}} k t}-\langle f, \varphi\rangle \mathrm{e}^{i \frac{2 \pi}{\mathcal{T}} k t} \mathrm{~d} t .
\end{aligned}
$$

We deduce that

$$
i \frac{2 \pi}{\mathcal{T}} k \int_{\mathbb{T}} u(t) \mathrm{e}^{i \frac{2 \pi}{\mathcal{T}} k t} \mathrm{~d} t=\int_{\mathbb{T}} g(t) \mathrm{e}^{i \frac{2 \pi}{\mathcal{T}} k t} \mathrm{~d} t
$$

as identity in $D_{0, \sigma}^{-1,2}\left(\mathrm{~B}_{N}\right)$. Since $k \in \mathbb{Z}$ is arbitrary, injectivity of the Fourier transform implies $-\partial_{t} u=g$.

From Lemma 6.4.1 and the embedding of $W^{1,1}\left(\mathbb{T} ; D_{0, \sigma}^{-1,2}\left(\mathrm{~B}_{N}\right)\right)$ in $C\left(\mathbb{T} ; D_{0, \sigma}^{-1,2}\left(\mathrm{~B}_{N}\right)\right)$, we have at this point already established a continuity property of the solution from Theorem 6.3.1. We can, however, improve on this property. For this purpose, we need the following lemma:

Lemma 6.4.2. Assume that $u \in L^{\infty}\left(\mathbb{T} ; L_{\sigma}^{2}\left(\mathbb{R}^{3}\right)\right)$ satisfies

$$
\forall N \in \mathbb{N}: \quad u \in W^{1,1}\left(\mathbb{T} ; D_{0, \sigma}^{-1,2}\left(\mathrm{~B}_{N}\right)\right) .
$$

Then there is a subset $\mathcal{N} \subset \mathbb{T}$ of measure zero such that

$$
\forall \varphi \in D_{0, \sigma}^{1,2}\left(\mathbb{R}^{3}\right) \forall s, t \in \mathbb{T} \backslash \mathcal{N}: \quad\langle u(t), \varphi\rangle-\langle u(s), \varphi\rangle=\int_{s}^{t}\left\langle\partial_{t} u(\tau), \varphi\right\rangle \mathrm{d} \tau
$$

and $u \in C\left(\mathbb{T} \backslash \mathcal{N} ; L_{w}^{2}\left(\mathbb{R}^{3}\right)^{3}\right)$, where $L_{w}^{2}\left(\mathbb{R}^{3}\right)^{3}$ denotes the space $L^{2}\left(\mathbb{R}^{3}\right)^{3}$ endowed with the weak topology.

Proof. It is well-known that for any function $u \in W^{1,1}\left(\mathbb{T} ; D_{0, \sigma}^{-1,2}\left(\mathrm{~B}_{N}\right)\right)$ there is a subset $\mathcal{N}_{N} \subset \mathbb{T}$ of measure zero such that

$$
\forall s, t \in \mathbb{T} \backslash \mathcal{N}_{N}: \quad u(t)-u(s)=\int_{s}^{t} \partial_{t} u(\tau) \mathrm{d} \tau
$$

as identify in $D_{0, \sigma}^{-1,2}\left(\mathrm{~B}_{N}\right)$. It follows that $u \in C\left(\mathbb{T} \backslash \mathcal{N}_{N} ; D_{0, \sigma}^{-1,2}\left(\mathrm{~B}_{N}\right)\right)$. We now put $\mathcal{N}:=\bigcup_{N \in \mathbb{N}} \mathcal{N}_{N}$. It is easy to see that (6.64) is a consequence of (6.65). We shall verify that $u \in C\left(\mathbb{T} \backslash \mathcal{N} ; L_{w}^{2}\left(\mathbb{R}^{3}\right)^{3}\right)$. For this purpose, let $h \in L^{2}\left(\mathbb{R}^{3}\right)^{3}$ and consider $u(t)$, which by assumption lies in $L_{\sigma}^{2}\left(\mathbb{R}^{3}\right)$, as a linear functional on $L^{2}\left(\mathbb{R}^{3}\right)^{3}$. According to the Helmholtz-Weyl decomposition (2.5), we can write $h$ as $h=H+\nabla p$ with $H \in L_{\sigma}^{2}\left(\mathbb{R}^{3}\right)$ 


\section{Weak solutions}

and $p \in W_{l o c}^{1,2}\left(\mathbb{R}^{3}\right)$. Let $\left\{\varphi_{n}\right\}_{n=1}^{\infty} \subset C_{0, \sigma}^{\infty}\left(\mathbb{R}^{3}\right)$ with $\lim _{n \rightarrow \infty} \varphi_{n}=H$ in $L^{2}\left(\mathbb{R}^{3}\right)^{3}$. For any $t \in \mathbb{T} \backslash \mathcal{N}$ and sequence $\left\{t_{m}\right\}_{m=1}^{\infty} \subset \mathbb{T} \backslash \mathcal{N}$ with $\lim _{m \rightarrow \infty} t_{m}=t$, we deduce

$$
\begin{aligned}
\left|\left\langle u\left(t_{m}\right), h\right\rangle-\langle u(t), h\rangle\right| & =\left|\left\langle u\left(t_{m}\right), H\right\rangle-\langle u(t), H\rangle\right| \\
& \leq\left|\left\langle u\left(t_{m}\right), H-\varphi_{n}\right\rangle\right|+\left|\left\langle u\left(t_{m}\right)-u(t), \varphi_{n}\right\rangle\right|+\left|\left\langle u(t), \varphi_{n}-H\right\rangle\right| \\
& \leq 2\|u\|_{L^{\infty}\left(\mathbb{T} ; L^{2}\left(\mathbb{R}^{3}\right)\right)}\left\|H-\varphi_{n}\right\|_{2}+\left\|u\left(t_{m}\right)-u(t)\right\|_{D_{0, \sigma}^{-1,2}\left(\mathrm{~B}_{N}\right)}\left|\varphi_{n}\right|_{1,2}
\end{aligned}
$$

provided $N$ is so large that $\operatorname{supp} \varphi_{n} \subset \mathrm{B}_{N}$. For arbitrary $\varepsilon>0$ we can now choose $n$ sufficiently large and subsequently utilize that $u \in C\left(\mathbb{T} \backslash \mathcal{N}_{N} ; D_{0, \sigma}^{-1,2}\left(\mathrm{~B}_{N}\right)\right)$ to deduce

$$
\left|\left\langle u\left(t_{m}\right), h\right\rangle-\langle u(t), h\rangle\right|<\varepsilon
$$

for sufficiently large $m$.

Finally, we can show that the solution $u$ from Theorem 6.3.1 can in fact be modified on the null set $\mathcal{N}$ in such a way that $u$ becomes continuous on the whole torus. Since $u=\mathcal{P} u+\mathcal{P}_{\perp} u$ and $\mathcal{P} u$ is time independent, if suffices to establish continuity only for $\mathcal{P}_{\perp} u$.

Theorem 6.4.3. Let $f \in L^{2}\left(\mathbb{T} ; D_{0}^{-1,2}\left(\mathbb{R}^{3}\right)^{3}\right)$ and $\lambda \geq 0$. The weak time-periodic solution $u \in L^{2}\left(\mathbb{T} ; D_{0, \sigma}^{1,2}\left(\mathbb{R}^{3}\right)\right)$ from Theorem 6.3 .1 can be modified on a set of measure zero such that

$$
\mathcal{P}_{\perp} u \in C\left(\mathbb{T} ; L_{w}^{2}\left(\mathbb{R}^{3}\right)^{3}\right),
$$

where again $L_{w}^{2}\left(\mathbb{R}^{3}\right)^{3}$ denotes the space $L^{2}\left(\mathbb{R}^{3}\right)^{3}$ endowed with the weak topology.

Proof. Theorem 6.3.1 yields $\mathcal{P}_{\perp} u \in L^{\infty}\left(\mathbb{T} ; L_{\sigma}^{2}\left(\mathbb{R}^{3}\right)\right)$. Thus, after possibly modifying $\mathcal{P}_{\perp} u$ on a set of measure zero, we may assume that $K:=\left\{\mathcal{P}_{\perp} u(t) \mid t \in \mathbb{T}\right\}$ is a bounded subset of $L_{\sigma}^{2}\left(\mathbb{R}^{3}\right)$. Consequently, by Alaoglu's theorem, $K$ is a compact subset of $L_{w}^{2}\left(\mathbb{R}^{3}\right)^{3}$. By Lemma 6.4.1, $\mathcal{P}_{\perp} u$ satisfies (6.63) and we can thus employ Lemma 6.4.2. We let $\mathcal{N}$ denote the null set from Lemma 6.4.2 and consider some $t \in \mathcal{N}$. Since $\mathcal{N}$ is a null set, there is a sequence $\left\{t_{n}\right\}_{n=1}^{\infty} \subset \mathbb{T} \backslash \mathcal{N}$ with $\lim _{n \rightarrow \infty} t_{n}=t$. Compactness of $K$ implies existence of an element $u_{\perp}^{t} \in L_{\sigma}^{2}\left(\mathbb{R}^{3}\right)$ and a subsequence of $\left\{t_{n}\right\}_{n=1}^{\infty}$, for simplicity still denoted $\left\{t_{n}\right\}_{n=1}^{\infty}$, such that $\lim _{n \rightarrow \infty} \mathcal{P}_{\perp} u\left(t_{n}\right)=u_{\perp}^{t}$ in $L_{w}^{2}\left(\mathbb{R}^{3}\right)^{3}$. Let $\left\{s_{m}\right\}_{m=1}^{\infty} \subset \mathbb{T} \backslash \mathcal{N}$ be another sequence with $\lim _{m \rightarrow \infty} s_{m}=t$. We shall verify that also $\lim _{m \rightarrow \infty} \mathcal{P}_{\perp} u\left(s_{m}\right)=u_{\perp}^{t}$ in $L_{w}^{2}\left(\mathbb{R}^{3}\right)^{3}$. Consider for this purpose an arbitrary $h \in L^{2}\left(\mathbb{R}^{3}\right)^{3}$, which by the HelmholtzWeyl decomposition (2.5) can be decomposed as $h=H+\nabla p$ with $H \in L_{\sigma}^{2}\left(\mathbb{R}^{3}\right)$ and $p \in W_{l o c}^{1,2}\left(\mathbb{R}^{3}\right)$. Let $\left\{\varphi_{k}\right\}_{k=1}^{\infty} \subset C_{0, \sigma}^{\infty}\left(\mathbb{R}^{3}\right)$ with $\lim _{k \rightarrow \infty} \varphi_{k}=H$ in $L^{2}\left(\mathbb{R}^{3}\right)^{3}$. We have

$$
\begin{aligned}
\left\langle\mathcal{P}_{\perp} u\left(s_{m}\right)-u_{\perp}^{t}, h\right\rangle= & \left\langle\mathcal{P}_{\perp} u\left(s_{m}\right)-u_{\perp}^{t}, H\right\rangle \\
= & \left\langle\mathcal{P}_{\perp} u\left(s_{m}\right)-u_{\perp}^{t}, H-\varphi_{k}\right\rangle \\
& +\left\langle\mathcal{P}_{\perp} u\left(s_{m}\right)-\mathcal{P}_{\perp} u\left(t_{n}\right), \varphi_{k}\right\rangle \\
& +\left\langle\mathcal{P}_{\perp} u\left(t_{n}\right)-u_{\perp}^{t}, \varphi_{k}\right\rangle .
\end{aligned}
$$


Let $\varepsilon>0$. Recalling (6.64) to estimate the second term on the right-hand side in (6.67), we choose first $k$ and then $n$ so large that

$$
\left|\left\langle\mathcal{P}_{\perp} u\left(s_{m}\right)-u_{\perp}^{t}, h\right\rangle\right|<\varepsilon
$$

for sufficiently large $m$. We conclude that $\lim _{m \rightarrow \infty} \mathcal{P}_{\perp} u\left(s_{m}\right)=u_{\perp}^{t}$ in $L_{w}^{2}\left(\mathbb{R}^{3}\right)^{3}$. We

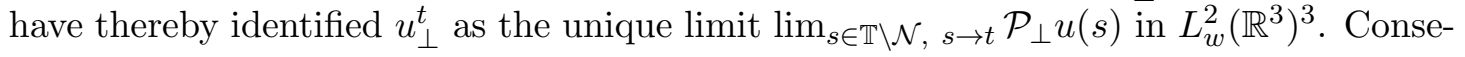
quently,

$$
u_{\perp}(t):= \begin{cases}\mathcal{P}_{\perp} u(t) & \text { if } t \in \mathbb{T} \backslash \mathcal{N}, \\ u_{\perp}^{t} & \text { if } t \in \mathcal{N}\end{cases}
$$

is well-defined. We verify that $u_{\perp} \in C\left(\mathbb{T} ; L_{w}^{2}\left(\mathbb{R}^{3}\right)^{3}\right)$. Let $t \in \mathbb{T}, h \in L^{2}\left(\mathbb{R}^{3}\right)^{3}$, and $\left\{t_{n}\right\}_{n=1}^{\infty} \subset \mathbb{T}$ with $\lim _{n \rightarrow \infty} t_{n}=t$. If $t_{n} \in \mathbb{T} \backslash \mathcal{N}$, put $\tilde{t}_{n}:=t_{n}$. If $t_{n} \in \mathcal{N}$, we choose $\tilde{t}_{n} \in \mathbb{T} \backslash \mathcal{N}$ such that $\left|\left\langle u_{\perp}\left(t_{n}\right)-u_{\perp}\left(\tilde{t}_{n}\right), h\right\rangle\right|<\frac{1}{n}$. Then

$$
\left|\left\langle u_{\perp}\left(t_{n}\right)-u_{\perp}(t), h\right\rangle\right| \leq\left|\left\langle u_{\perp}\left(t_{n}\right)-u_{\perp}\left(\tilde{t}_{n}\right), h\right\rangle\right|+\left|\left\langle u_{\perp}\left(\tilde{t}_{n}\right)-u_{\perp}(t), h\right\rangle\right| \rightarrow 0 \quad \text { as } n \rightarrow \infty .
$$

Hence, $u_{\perp} \in C\left(\mathbb{T} ; L_{w}^{2}\left(\mathbb{R}^{3}\right)^{3}\right)$. Since $u_{\perp}$ is a modification of $\mathcal{P}_{\perp} u$ on a null set, we conclude the lemma.

Remark 6.4.4. It was originally shown by HopF in [32] that the weak solution to the Navier-Stokes initial-value problem established by LERAY in [42] belongs to the space $C\left([0, T) ; L_{w}^{2}\left(\mathbb{R}^{3}\right)^{3}\right)$. The proof Theorem 6.4 .3 follows essentially the proof in [32]; see also [19, Lemma 2.2].

\subsection{Existence of a pressure}

We end the chapter by constructing a pressure term $\mathfrak{p}$ associated to the weak solution to (6.1) from Theorem 6.3.1. The existence of such a pressure term implies that the weak solution is in fact a solution in a standard sense of distributions.

Two methods for constructing the pressure will be given. The first method presented in Theorem 6.5.1 below is very general. It requires no more regularity on the data than was needed in Theorem 6.3.1 to show existence of a weak solution. Moreover, it does not exploit that (6.1) is a whole-space problem and can thus easily be adapted to more general domains. It does, however, only produce a pressure with very little regularity in the time variable. In contrast, in Theorem 6.5.3 we assume more regularity on the data, and take advantage of the possibility in the whole-space case to express the pressure in terms of an explicit formula. We then employ the linear theory from Chapter 3 to construct a pressure that is more regular.

Theorem 6.5.1. Let $f \in L^{2}\left(\mathbb{T} ; D_{0}^{-1,2}\left(\mathbb{R}^{3}\right)^{3}\right)$ and $\lambda \geq 0$. Let $u \in L^{2}\left(\mathbb{T} ; D_{0, \sigma}^{1,2}\left(\mathbb{R}^{3}\right)\right)$ be a weak solution to (6.1) that satisfies

$$
\begin{aligned}
& \mathcal{P} u \in D_{0, \sigma}^{1,2}\left(\mathbb{R}^{3}\right), \\
& \mathcal{P}_{\perp} u \in L^{2}\left(\mathbb{T} ; D_{0, \sigma}^{1,2}\left(\mathbb{R}^{3}\right)\right) \cap L^{\infty}\left(\mathbb{T} ; L_{\sigma}^{2}\left(\mathbb{R}^{3}\right)\right) .
\end{aligned}
$$




\section{Weak solutions}

Then there $i s^{3}$

$$
\mathfrak{p} \in \mathcal{D}^{\prime}\left(\mathbb{R}^{3} \times \mathbb{T}\right)
$$

such that

$$
\begin{aligned}
& \forall \Phi \in C_{0}^{\infty}\left(\mathbb{R}^{3} \times \mathbb{T}\right)^{3}: \\
& \qquad \int_{\mathbb{T}} \int_{\mathbb{R}^{3}}-u \cdot \partial_{t} \Phi+\nabla u: \nabla \Phi-\lambda \partial_{1} u \cdot \Phi+(u \cdot \nabla u) \cdot \Phi \mathrm{d} x \mathrm{~d} t=\int_{\mathbb{T}}\langle f, \Phi\rangle \mathrm{d} t-\langle\nabla \mathfrak{p}, \Phi\rangle .
\end{aligned}
$$

Remark 6.5.2. We use $\mathcal{D}^{\prime}\left(\mathbb{R}^{3} \times \mathbb{T}\right)$ to denote the dual space of $\mathcal{D}\left(\mathbb{R}^{3} \times \mathbb{T}\right)$, which is defined as the vector space $C_{0}^{\infty}\left(\mathbb{R}^{3} \times \mathbb{T}\right)$ equipped with the canonical inductive limit topology. In other words, $\mathcal{D}^{\prime}\left(\mathbb{R}^{3} \times \mathbb{T}\right)$ is the space of distributions on $\mathbb{R}^{3} \times \mathbb{T}$. For $\mathfrak{p} \in \mathcal{D}^{\prime}\left(\mathbb{R}^{3} \times \mathbb{T}\right)$ the gradient $\nabla p$ is defined in the standard distributional sense. We shall not need specific properties of the space $\mathcal{D}^{\prime}\left(\mathbb{R}^{3} \times \mathbb{T}\right)$ as it is only used to characterize the pressure in (6.70).

Proof of Theorem 6.5.1. In the proof we shall utilize the Fourier transform. The function spaces in the following will therefore all be complexified. As a consequence, the pressure term $\mathfrak{p}$ that will be constructed may be a complex-valued distribution. It is obvious, however, that the real part of $\mathfrak{p}$ will also satisfy (6.71).

We proceed with the proof. Put $v:=\mathcal{P} u$ and $w:=\mathcal{P}_{\perp} u$. As was already observed in the proof of Lemma 4.4.4, the fact that $u$ is a weak solution to (6.1) implies that $w$ satisfies

$$
\begin{aligned}
& \forall \Phi \in C_{0, \sigma}^{\infty}\left(\mathbb{R}^{3} \times \mathbb{T}\right): \\
& \qquad \begin{array}{l}
\int_{\mathbb{T}} \int_{\mathbb{R}^{3}}-w \cdot \partial_{t} \Phi+\nabla w: \nabla \Phi-\lambda \partial_{1} w \cdot \Phi \\
\quad+\left(\mathcal{P}_{\perp}[w \cdot \nabla w]+w \cdot \nabla v+v \cdot \nabla w\right) \cdot \Phi \mathrm{d} x \mathrm{~d} t=\int_{\mathbb{T}}\left\langle\mathcal{P}_{\perp} f, \Phi\right\rangle \mathrm{d} t
\end{array}
\end{aligned}
$$

and $v$ satisfies

$$
\begin{aligned}
& \forall \varphi \in C_{0, \sigma}^{\infty}\left(\mathbb{R}^{3}\right): \\
& \qquad \int_{\mathbb{R}^{3}} \nabla v: \nabla \varphi-\lambda \partial_{1} v \cdot \varphi+(v \cdot \nabla v+\mathcal{P}[w \cdot \nabla w]) \cdot \varphi \mathrm{d} x \mathrm{~d} t=\langle\mathcal{P} f, \varphi\rangle .
\end{aligned}
$$

We shall construct a pressure term for the weak formulations (6.72) and (6.73) separately.

${ }^{3}$ See Remark 6.5.2 for definition of the space $\mathcal{D}^{\prime}\left(\mathbb{R}^{3} \times \mathbb{T}\right)$. 
We start with (6.72). Observe that

$$
\begin{aligned}
\int_{\mathbb{T}}|w \cdot \nabla w|_{-1,2} \mathrm{~d} t & =\int_{\mathbb{T}} \sup _{\substack{\varphi \in C_{0}^{\infty}\left(\mathbb{R}^{3}\right)^{3} \\
|\varphi|_{1,2} \leq 1}}\left|\int_{\mathbb{R}^{3}}(w \cdot \nabla w) \cdot \varphi \mathrm{d} x\right| \mathrm{d} t \\
& =\int_{\mathbb{T}} \sup _{\substack{\varphi \in C_{0}^{\infty}\left(\mathbb{R}^{3}\right)^{3} \\
|\varphi|_{1,2} \leq 1}}\left|\int_{\mathbb{R}^{3}} w \otimes w: \nabla \varphi \mathrm{d} x\right| \mathrm{d} t \\
& \leq c_{1} \int_{\mathbb{T}}\|w(t)\|_{4}^{2} \mathrm{~d} t \\
& \leq c_{2} \int_{\mathbb{T}}\|w(t)\|_{2}^{\frac{1}{2}}\|\nabla w(t)\|_{2}^{\frac{3}{2}} \mathrm{~d} t \\
& \leq c_{3}\|w\|_{L^{\infty}\left(\mathbb{T} ; L^{2}\left(\mathbb{R}^{3}\right)\right)}^{\frac{1}{2}}\|w\|_{L^{2}\left(\mathbb{T} ; D_{0, \sigma}^{1,2}\left(\mathbb{R}^{3}\right)\right)},
\end{aligned}
$$

where the second-last inequality is due to Lemma 2.3.1. It follows that

$$
w \cdot \nabla w \in L^{1}\left(\mathbb{T} ; D_{0}^{-1,2}\left(\mathbb{R}^{3}\right)^{3}\right) .
$$

By a similar computation we find that

$$
\begin{aligned}
\int_{\mathbb{T}}|w \cdot \nabla v|_{-1,2} \mathrm{~d} t & =\int_{\mathbb{T}} \sup _{\substack{\varphi \in C_{0}^{\infty}\left(\mathbb{R}^{3}\right)^{3} \\
|\varphi|_{1,2} \leq 1}}\left|\int_{\mathbb{R}^{3}} v \otimes w: \nabla \varphi \mathrm{d} x\right| \mathrm{d} t \\
& \leq c_{4} \int_{\mathbb{T}}\|v\|_{6}\|w(t)\|_{3} \mathrm{~d} t \\
& \leq c_{5}\|\nabla v\|_{2} \int_{\mathbb{T}}\|w(t)\|_{2}^{\frac{1}{2}}\|\nabla w(t)\|_{2}^{\frac{1}{2}} \mathrm{~d} t \\
& \leq c_{6}|v|_{1,2}\|w\|_{L^{\infty}\left(\mathbb{T} ; L^{2}\left(\mathbb{R}^{3}\right)\right)}^{\frac{1}{2}}\|w\|_{L^{2}\left(\mathbb{T} ; D_{0, \sigma}^{1,2}\left(\mathbb{R}^{3}\right)\right)}
\end{aligned}
$$

and thus

$$
w \cdot \nabla v \in L^{1}\left(\mathbb{T} ; D_{0}^{-1,2}\left(\mathbb{R}^{3}\right)^{3}\right)
$$

Analogously, we deduce

$$
v \cdot \nabla w \in L^{1}\left(\mathbb{T} ; D_{0}^{-1,2}\left(\mathbb{R}^{3}\right)^{3}\right) .
$$

We now expand $u \in L^{2}\left(\mathbb{T} ; D_{0, \sigma}^{1,2}\left(\mathbb{R}^{3}\right)\right)$ in a Fourier series

$$
\left\{u_{k}\right\}_{k \in \mathbb{Z}} \in \ell^{2}\left(\mathbb{Z} ; D_{0, \sigma}^{1,2}\left(\mathbb{R}^{3}\right)\right) .
$$

Observe that $v=u_{0}$. Consequently, $\left\{u_{k}\right\}_{k \neq 0}$ is the Fourier series of $w$, whence

$$
\left\{u_{k}\right\}_{k \neq 0} \in \ell^{2}\left(\mathbb{Z} ; L^{2}\left(\mathbb{R}^{3}\right)^{3}\right) .
$$




\section{Weak solutions}

We further put ${ }^{4}$

$$
H:=\mathcal{P}_{\perp}[u \cdot \nabla u]=\mathcal{P}_{\perp}[w \cdot \nabla w]+w \cdot \nabla v+v \cdot \nabla w
$$

In view of $(6.74)-(6.76), H \in L^{1}\left(\mathbb{T} ; D_{0}^{-1,2}\left(\mathbb{R}^{3}\right)^{3}\right)$. We can thus expand $H$ into a Fourier series

$$
\left\{H_{k}\right\}_{k \in \mathbb{Z}} \in \ell^{\infty}\left(\mathbb{Z} ; D_{0}^{-1,2}\left(\mathbb{R}^{3}\right)^{3}\right) .
$$

Observe that $H_{0}=\mathcal{F}_{\mathbb{T}}[H](0)=\mathcal{P} H=0$. Finally, we also expand $f$ into a Fourier series

$$
\left\{f_{k}\right\}_{k \in \mathbb{Z}} \in \ell^{2}\left(\mathbb{Z} ; D_{0}^{-1,2}\left(\mathbb{R}^{3}\right)^{3}\right) .
$$

For $k \in \mathbb{Z} \backslash\{0\}$ and $N \in \mathbb{N}$ define

$$
\begin{aligned}
& \mathcal{L}_{k}^{N}: W_{0}^{1,2}\left(\mathrm{~B}_{N}\right)^{3} \rightarrow \mathbb{C}, \\
& \mathcal{L}_{k}^{N}(\varphi):=\int_{\mathbb{R}^{3}}-i k \frac{2 \pi}{\mathcal{T}} u_{-k} \cdot \varphi+\nabla u_{-k}: \nabla \varphi-\lambda \partial_{1} u_{-k} \cdot \varphi \mathrm{d} x+\left\langle H_{-k}, \varphi\right\rangle-\left\langle f_{-k}, \varphi\right\rangle .
\end{aligned}
$$

Recalling that $u_{k}=\mathcal{F}_{\mathbb{T}}[w](k)$ and thus $u_{k} \in L^{2}\left(\mathbb{R}^{3}\right)^{3}$, it is easy to see that $\mathcal{L}_{k}^{N}$ is a bounded linear functional on $W_{0}^{1,2}\left(\mathrm{~B}_{N}\right)^{3}$. Consider now an element $\varphi \in C_{0, \sigma}^{\infty}\left(\mathbb{R}^{3}\right)$. The function $\Phi(x, t):=\varphi(x) \mathrm{e}^{\frac{2 \pi}{T} k t}$ is then admissible as test function in (6.72), which implies that $\mathcal{L}_{k}^{N}(\varphi)=0$. Thus $\mathcal{L}_{k}^{N}$ vanishes on $C_{0, \sigma}^{\infty}\left(\mathbb{R}^{3}\right)$. By a well-known theorem, see for example [17, Corollary III.5.1] which easily extends by linearity to complexified vector spaces, it follows that there is $\mathfrak{p}_{k}^{N} \in L^{2}\left(\mathrm{~B}_{N}\right)$ such that $\mathcal{L}_{k}^{N}=\nabla \mathfrak{p}_{k}^{N}$, that is,

$$
\forall \varphi \in C_{0}^{\infty}\left(\mathrm{B}_{N}\right)^{3}: \quad \mathcal{L}_{k}^{N}(\varphi)=-\int_{\mathbb{R}^{3}} \mathfrak{p}_{k}^{N} \cdot \operatorname{div} \varphi \mathrm{d} x .
$$

Clearly, $\mathfrak{p}_{k}^{N}$ can be chosen such that

$$
\int_{\mathrm{B}_{N}} \mathfrak{p}_{k}^{N} \mathrm{~d} x=0
$$

Consequently, see for example [17, Theorem III.3.1], there is a $\varphi \in W_{0}^{1,2}\left(\mathrm{~B}_{N}\right)^{3}$ with the properties $\operatorname{div} \varphi=-\mathfrak{p}_{k}^{N}$ and $|\varphi|_{1,2} \leq c_{7}\left\|\mathfrak{p}_{k}^{N}\right\|_{2}$. Inserting this particular $\varphi$ in (6.82), we find that $\left\|\mathfrak{p}_{k}^{N}\right\|_{2}^{2}=\mathcal{L}_{k}^{N}(\varphi)$. It follows that

$$
\left\|\mathfrak{p}_{k}^{N}\right\|_{2} \leq c_{8}\left(|k|\left\|u_{-k}\right\|_{2}+\left\|\nabla u_{-k}\right\|_{2}+\left|H_{-k}\right|_{-1,2}+\left|f_{-k}\right|_{-1,2}\right)
$$

\footnotetext{
${ }^{4}$ The definition of $H$ requires that we identify $u \cdot \nabla u$ as an element of a function space on which $\mathcal{P}_{\perp}$ is well-defined. For this purpose we may, for example, choose $L^{1}\left(\mathbb{T} ; W_{0}^{-1,2}\left(\mathbb{R}^{3}\right)^{3}\right)$.
} 
with $c_{8}=c_{8}(N)$. Recalling (6.77)-(6.80), we see that

$$
\left\{\frac{1}{k} \mathfrak{p}_{k}^{N}\right\}_{k \neq 0} \in \ell^{2}\left(\mathbb{Z} ; L^{2}\left(\mathrm{~B}_{N}\right)\right)
$$

We can thus define

$$
\mathfrak{P}^{N}:=-i \frac{\mathcal{T}}{2 \pi} \mathcal{F}_{\mathbb{T}}^{-1}\left[\left\{\frac{1}{k} \mathfrak{p}_{k}^{N}\right\}_{k \neq 0}\right] \in L^{2}\left(\mathbb{T} ; L^{2}\left(\mathrm{~B}_{N}\right)\right) .
$$

Consider now $N, M \in \mathbb{N}$ with $N<M$. For any $\varphi \in C_{0}^{\infty}\left(\mathrm{B}_{N}\right)^{3}$ it follows from (6.82) that $\left\langle\mathfrak{P}^{N}(\cdot), \operatorname{div} \varphi\right\rangle_{L^{2}\left(\mathrm{~B}_{N}\right)}=\left\langle\mathfrak{P}^{M}(\cdot), \operatorname{div} \varphi\right\rangle_{L^{2}\left(\mathrm{~B}_{N}\right)}$ as equality in $L^{2}(\mathbb{T} ; \mathbb{C})$. Consequently, $\nabla \mathfrak{P}^{N}(t)=\nabla \mathfrak{P}^{M}(t)$ in $D_{0}^{-1,2}\left(\mathrm{~B}_{N}\right)^{3}$ for almost all $t \in \mathbb{T}$. We therefore find that $\mathfrak{P}^{N}(t)=$ $\mathfrak{P}^{M}(t)+c(N, M, t)$ in $L^{2}\left(\mathrm{~B}_{N}\right)$ for some function $c(N, M, \cdot) \in L^{2}(\mathbb{T})$. Letting

$$
\widetilde{\mathfrak{P}}^{1}(t):=\mathfrak{P}^{1}(t), \quad \widetilde{\mathfrak{P}}^{N+1}(t):=\mathfrak{P}^{N+1}(t)+c(N, N+1, t)+\left(\widetilde{\mathfrak{P}}^{N}(t)-\mathfrak{P}^{N}(t)\right),
$$

we inductively obtain a sequence $\left\{\widetilde{\mathfrak{P}}^{N}\right\}_{N=1}^{\infty}$ with $\widetilde{\mathfrak{P}}^{M}=\widetilde{\mathfrak{P}}^{N}$ in $L^{2}\left(\mathbb{T} ; L^{2}\left(\mathrm{~B}_{N}\right)\right)$ for $M \geq$ $N$. At this point, we can define an element in $\mathfrak{P} \in L^{2}\left(\mathbb{T} ; L_{\text {loc }}^{2}\left(\mathbb{R}^{3}\right)\right)$ via $\mathfrak{P}(t, x):=$ $\widetilde{\mathfrak{P}}^{N}(t, x)$ if $|x| \leq N$. We can consider $\mathfrak{P}$ as an element in $\mathcal{D}^{\prime}\left(\mathbb{R}^{3} \times \mathbb{T}\right)$ and put

$$
\pi:=\partial_{t} \mathfrak{P} \in \mathcal{D}^{\prime}\left(\mathbb{R}^{3} \times \mathbb{T}\right) .
$$

We shall now verify that $\pi$ as defined above is a valid pressure term corresponding to the weak formulation (6.72). For this purpose consider $\Phi \in C_{0}^{\infty}\left(\mathbb{R}^{3} \times \mathbb{T}\right)^{3}$ and observe that

$$
\begin{aligned}
\langle\nabla \pi, \Phi\rangle=\left\langle\mathfrak{P}, \partial_{t} \operatorname{div} \Phi\right\rangle & =\int_{\mathbb{T}} \int_{\mathbb{R}^{3}} \mathfrak{P}(x, t) \cdot \partial_{t} \operatorname{div} \Phi(x, t) \mathrm{d} x \mathrm{~d} t \\
& =\int_{\mathbb{T}} \int_{\mathbb{R}^{3}} \mathfrak{P}^{N}(x, t) \cdot \partial_{t} \operatorname{div} \Phi(x, t) \mathrm{d} x \mathrm{~d} t
\end{aligned}
$$

for $N \in \mathbb{N}$ with $\operatorname{supp}(\Phi) \subset \mathrm{B}_{N} \times \mathbb{T}$. Recalling (6.83), it follows that

$$
\langle\nabla \pi, \Phi\rangle=-i \frac{\mathcal{T}}{2 \pi} \int_{\mathbb{T}}\left\langle\mathcal{F}_{\mathbb{T}}^{-1}\left[\left\{\frac{1}{k} \mathfrak{p}_{k}^{N}\right\}_{k \neq 0}\right], \overline{\partial_{t} \operatorname{div} \Phi(\cdot, t)}\right\rangle_{L^{2}\left(\mathrm{~B}_{N}\right)} \mathrm{d} t .
$$

Recall that we in this proof regard $L^{2}\left(\mathrm{~B}_{N}\right)$ as a vector space over $\mathbb{C}$, which is the reason the complex conjugation of $\partial_{t} \operatorname{div} \Phi(x, t)$ appears in the equation above. By Parseval's identity, we deduce that

$$
\langle\nabla \pi, \Phi\rangle=\sum_{k \in \mathbb{Z} \backslash\{0\}}-i \frac{\mathcal{T}}{2 \pi}\left\langle\frac{1}{k} \mathfrak{p}_{k}^{N},-i \frac{2 \pi}{\mathcal{T}} k \operatorname{div} \bar{\Phi}_{k}\right\rangle_{L^{2}\left(\mathrm{~B}_{N}\right)},
$$




\section{Weak solutions}

where $\left\{\Phi_{k}\right\}_{k \in \mathbb{Z}} \in \ell^{2}\left(\mathbb{Z} ; L^{2}\left(\mathbb{R}^{3}\right)^{3}\right)$ is the Fourier transform $\mathcal{F}_{\mathbb{T}}[\Phi]$ of $\Phi$ with respect to the $t$-variable. Clearly, $\Phi_{k}$ enjoys the same regularity as $\Phi$ with respect to the $x$-variable. In other words, $\Phi_{k} \in C_{0}^{\infty}\left(\mathbb{R}^{3}\right)^{3}$. Recalling (6.82), we therefore see that

$$
\begin{aligned}
\langle\nabla \pi, \Phi\rangle= & \sum_{k \in \mathbb{Z} \backslash\{0\}}\left\langle\mathfrak{p}_{k}^{N},-\operatorname{div} \bar{\Phi}_{k}\right\rangle_{L^{2}\left(\mathrm{~B}_{N}\right)} \\
= & \sum_{k \in \mathbb{Z} \backslash\{0\}} \mathcal{L}_{k}^{N}\left(\Phi_{k}\right) \\
= & \sum_{k \in \mathbb{Z} \backslash\{0\}} \int_{\mathbb{R}^{3}}-i k \frac{2 \pi}{\mathcal{T}} u_{-k} \cdot \Phi_{k}+\nabla u_{-k}: \nabla \Phi_{k}-\lambda \partial_{1} u_{-k} \cdot \Phi_{k} \mathrm{~d} x \\
& \quad+\left\langle H_{-k}, \Phi_{k}\right\rangle-\left\langle f_{-k}, \Phi_{k}\right\rangle .
\end{aligned}
$$

A Parseval-type identity holds for all terms on the right-hand side above. For example, utilizing Fubini's theorem and the fact that the Fourier series $\sum_{k \in \mathbb{Z} \backslash\{0\}} \Phi_{k} \mathrm{e}^{i \frac{2 \pi}{T} k t}$ converges pointwise for each $t \in \mathbb{T}$ towards $\mathcal{P}_{\perp} \Phi$ in the topology of $D_{0}^{1,2}\left(\mathbb{R}^{3}\right)$, we obtain

$$
\begin{aligned}
\sum_{k \in \mathbb{Z} \backslash\{0\}}\left\langle H_{-k}, \Phi_{k}\right\rangle & =\sum_{k \in \mathbb{Z} \backslash\{0\}}\left\langle\int_{\mathbb{T}} H(t) \mathrm{e}^{i \frac{2 \pi}{\mathcal{T}} k t} \mathrm{~d} t, \Phi_{k}\right\rangle \\
& =\int_{\mathbb{T}}\left\langle H(t), \sum_{k \in \mathbb{Z} \backslash\{0\}} \Phi_{k} \mathrm{e}^{i \frac{2 \pi}{\mathcal{T}} k t}\right\rangle \mathrm{d} t=\int_{\mathbb{T}}\left\langle H(t), \mathcal{P}_{\perp} \Phi\right\rangle \mathrm{d} t .
\end{aligned}
$$

Recalling that $\mathcal{P} H=0$, the above identity implies

$$
\begin{aligned}
\sum_{k \in \mathbb{Z} \backslash\{0\}}\left\langle H_{-k}, \Phi_{k}\right\rangle & =\int_{\mathbb{T}}\langle H(t), \Phi\rangle \mathrm{d} t \\
& =\int_{\mathbb{T}} \int_{\mathbb{R}^{3}}\left(\mathcal{P}_{\perp}[w \cdot \nabla w]+w \cdot \nabla v+v \cdot \nabla w\right) \cdot \Phi(x, t) \mathrm{d} x \mathrm{~d} t .
\end{aligned}
$$

Applying a similar computation in the other terms on the right-hand side in (6.85), we obtain

$$
\begin{aligned}
\langle\nabla \pi, \Phi\rangle=\int_{\mathbb{T}} \int_{\mathbb{R}^{3}}- & w \cdot \partial_{t} \Phi+\nabla w: \nabla \Phi-\lambda \partial_{1} w \cdot \Phi \\
& +\left(\mathcal{P}_{\perp}[w \cdot \nabla w]+w \cdot \nabla v+v \cdot \nabla w\right) \cdot \Phi \mathrm{d} x \mathrm{~d} t-\int_{\mathbb{T}}\left\langle\mathcal{P}_{\perp} f, \Phi\right\rangle \mathrm{d} t .
\end{aligned}
$$

We have thus constructed in $\pi$ a pressure term for the weak formulation (6.72). The existence of a pressure term for the weak formulation (6.73) follows a by standard argument. More specifically, define on the real-valued vector space $C_{0}^{\infty}\left(\mathbb{R}^{3}\right)^{3}$ the linear 
functional

$$
\begin{aligned}
& \mathcal{L}: C_{0}^{\infty}\left(\mathbb{R}^{3}\right)^{3} \rightarrow \mathbb{R} \\
& \mathcal{L}(\varphi):=\int_{\mathbb{R}^{3}} \nabla v: \nabla \varphi-\lambda \partial_{1} v \cdot \varphi+(v \cdot \nabla v+\mathcal{P}[w \cdot \nabla w]) \cdot \varphi \mathrm{d} x \mathrm{~d} t-\langle\mathcal{P} f, \varphi\rangle .
\end{aligned}
$$

In view of (6.68) and (6.74), it is standard to show for each bounded domain $\Omega \subset$ $\mathbb{R}^{3}$ that the restriction $\mathcal{L}_{\mid C_{0}^{\infty}(\Omega)^{3}}$ extends to a bounded linear functional on $D_{0}^{1,2}(\Omega)^{3}$. Consequently, see for example [17, Corollary III.5.1], there is $p \in L_{\text {loc }}^{2}\left(\mathbb{R}^{3}\right)$ such that $\mathcal{L}=\nabla p$ in the sense of distributions. We can trivially extend $p$ to a time-independent element of $L_{\text {loc }}^{2}\left(\mathbb{R}^{3} \times \mathbb{T}\right)$. Then $\nabla p \in \mathcal{D}^{\prime}\left(\mathbb{R}^{3} \times \mathbb{T}\right)^{3}$ and for each $\Phi \in C_{0}^{\infty}\left(\mathbb{R}^{3} \times \mathbb{T}\right)^{3}$ we have $\left\langle\nabla p, \mathcal{P}_{\perp} \Phi\right\rangle=0$. Thus,

$$
\begin{aligned}
\langle\nabla p, \Phi\rangle & =\langle\nabla p, \mathcal{P} \Phi\rangle \\
& =\int_{\mathbb{R}^{3}} \nabla v: \nabla \mathcal{P} \Phi-\lambda \partial_{1} v \cdot \mathcal{P} \Phi+(v \cdot \nabla v+\mathcal{P}[w \cdot \nabla w]) \cdot \mathcal{P} \Phi \mathrm{d} x \mathrm{~d} t-\langle\mathcal{P} f, \mathcal{P} \Phi\rangle .
\end{aligned}
$$

We finally put

$$
\mathfrak{p}:=\pi+p
$$

and observe for arbitrary $\Phi \in C_{0}^{\infty}\left(\mathbb{R}^{3} \times \mathbb{T}\right)^{3}$, by recalling $u=v+w$ and (6.86)-(6.87), that

$$
\begin{aligned}
& \int_{\mathbb{T}} \int_{\mathbb{R}^{3}}- u \cdot \partial_{t} \Phi+\nabla u: \nabla \Phi-\lambda \partial_{1} u \cdot \Phi+(u \cdot \nabla u) \cdot \Phi \mathrm{d} x \mathrm{~d} t-\int_{\mathbb{T}}\langle f, \Phi\rangle \mathrm{d} t \\
&= \int_{\mathbb{T}} \int_{\mathbb{R}^{3}}-(v+w) \cdot \partial_{t} \Phi+(\nabla v+\nabla w): \nabla \Phi-\lambda\left(\partial_{1} v+\partial_{1} w\right) \cdot \Phi \\
& \quad+((v+w) \cdot \nabla(v+w)) \cdot \Phi \mathrm{d} x \mathrm{~d} t \\
&-\int_{\mathbb{T}}\left\langle\mathcal{P}_{\perp} f, \Phi\right\rangle \mathrm{d} t-\int_{\mathbb{T}}\langle\mathcal{P} f, \Phi\rangle \mathrm{d} t \\
&= \int_{\mathbb{T}} \int_{\mathbb{R}^{3}}-w \cdot \partial_{t} \Phi+\nabla w: \nabla \Phi-\lambda \partial_{1} w \cdot \Phi \\
& \quad+\left(\mathcal{P}_{\perp}[w \cdot \nabla w]+w \cdot \nabla v+v \cdot \nabla w\right) \cdot \Phi \mathrm{d} x \mathrm{~d} t-\int_{\mathbb{T}}\left\langle\mathcal{P}_{\perp} f, \Phi\right\rangle \mathrm{d} t \\
& \quad+\int_{\mathbb{R}^{3}} \nabla v: \nabla \mathcal{P} \Phi-\lambda \partial_{1} v \cdot \mathcal{P} \Phi+(v \cdot \nabla v+\mathcal{P}[w \cdot \nabla w]) \cdot \mathcal{P} \Phi \mathrm{d} x \mathrm{~d} t-\langle\mathcal{P} f, \mathcal{P} \Phi\rangle \\
&=\langle\nabla \pi, \Phi\rangle+\langle\nabla p, \Phi\rangle \\
&=\langle\nabla \mathfrak{p}, \Phi\rangle .
\end{aligned}
$$

We have thus established (6.71) and thereby the theorem. 


\section{Weak solutions}

In the next theorem we show existence of a more regular pressure by assuming more regularity on the data $f$. In contrast to Theorem 6.5.1, the proof of Theorem 6.5.3 below utilizes the linear theory from Chapter 3, in particular Lemma 3.6.8. Consequently, Theorem 6.5.3 is less adaptable to more general domains than Theorem 6.5.1.

Theorem 6.5.3. Let $\lambda \geq 0$ and $u \in L^{2}\left(\mathbb{T} ; D_{0, \sigma}^{1,2}\left(\mathbb{R}^{3}\right)\right)$ be a weak solution to (6.1) that satisfies

$$
\begin{aligned}
& \mathcal{P} u \in D_{0, \sigma}^{1,2}\left(\mathbb{R}^{3}\right), \\
& \mathcal{P}_{\perp} u \in L^{2}\left(\mathbb{T} ; D_{0, \sigma}^{1,2}\left(\mathbb{R}^{3}\right)\right) \cap L^{\infty}\left(\mathbb{T} ; L_{\sigma}^{2}\left(\mathbb{R}^{3}\right)\right) .
\end{aligned}
$$

If for some $q \in\left(1, \frac{5}{4}\right]$

$$
f \in L^{q}\left(\mathbb{T} \times \mathbb{R}^{3}\right)^{3}+L^{\frac{3}{2}}\left(\mathbb{T} \times \mathbb{R}^{3}\right)^{3},
$$

then there is a pressure

$$
\begin{aligned}
& \mathfrak{p} \in L^{q}\left(\mathbb{T} ; L^{\frac{3 q}{3-q}}\left(\mathbb{R}^{3}\right)\right)+L^{\frac{3}{2}}\left(\mathbb{T} ; L^{3}\left(\mathbb{R}^{3}\right)\right), \\
& \nabla \mathfrak{p} \in L^{q}\left(\mathbb{T} \times \mathbb{R}^{3}\right)^{3}+L^{\frac{3}{2}}\left(\mathbb{T} \times \mathbb{R}^{3}\right)^{3}
\end{aligned}
$$

such that $(u, \mathfrak{p})$ is a solution to (6.1) in the standard sense of distributions, that is, for all $\Phi \in C_{0}^{\infty}\left(\mathbb{T} \times \mathbb{R}^{3}\right)^{3}$ :

$$
\int_{\mathbb{T}} \int_{\mathbb{R}^{3}}-u \cdot \partial_{t} \Phi+\nabla u: \nabla \Phi+\nabla \mathfrak{p} \cdot \Phi-\lambda \partial_{1} u \cdot \Phi+(u \cdot \nabla u) \cdot \Phi \mathrm{d} x \mathrm{~d} t=\int_{\mathbb{T}} \int_{\mathbb{R}^{3}} f \cdot \Phi \mathrm{d} x \mathrm{~d} t .
$$

If $\lambda>0$ and for some $q \in\left(1, \frac{5}{4}\right]$

$$
f \in L^{q}\left(\mathbb{T} \times \mathbb{R}^{3}\right)^{3} \cap L^{\frac{3}{2}}\left(\mathbb{T} \times \mathbb{R}^{3}\right)^{3},
$$

then there is a pressure

$$
\mathfrak{p} \in L^{q}\left(\mathbb{T} ; L^{\frac{3 q}{3-q}}\left(\mathbb{R}^{3}\right)\right), \quad \nabla \mathfrak{p} \in L^{q}\left(\mathbb{T} \times \mathbb{R}^{3}\right)^{3}
$$

such that $(u, \mathfrak{p})$ is a solution to (6.1) in the standard sense of distributions.

Proof. Put $v:=\mathcal{P} u$ and $w:=\mathcal{P}_{\perp} u$. By Hölder's inequality, (2.2), and (6.88)-(6.89), it follows that

$$
\int_{\mathbb{T}} \int_{\mathbb{R}^{3}}|v \cdot \nabla w|^{\frac{3}{2}} \mathrm{~d} x \mathrm{~d} t \leq \int_{\mathbb{T}}\|v\|_{6}^{\frac{3}{2}}\|\nabla w(t)\|_{2}^{\frac{3}{2}} \mathrm{~d} t \leq c_{1}\|v\|_{D_{0, \sigma}^{1,2}\left(\mathbb{R}^{3}\right)}^{\frac{3}{2}}\|w\|_{L^{2}\left(\mathbb{T} ; D_{0, \sigma}^{1,2}\left(\mathbb{R}^{3}\right)\right)}<\infty .
$$

Hence $v \cdot \nabla w \in L^{\frac{3}{2}}\left(\mathbb{T} \times \mathbb{R}^{3}\right)^{3}$. Similarly, we also verify that $v \cdot \nabla v \in L^{\frac{3}{2}}\left(\mathbb{T} \times \mathbb{R}^{3}\right)^{3}$. Recalling Lemma 3.2.3 and the decomposition (3.20), we can apply the Helmholtz projection to both these terms and deduce that

$$
\left[\mathrm{Id}-\mathcal{P}_{H}\right](v \cdot \nabla v+v \cdot \nabla w) \in \mathscr{G}^{\frac{3}{2}}\left(\mathbb{T} \times \mathbb{R}^{3}\right) .
$$


Thus, by Lemma 3.6.8 there is $\mathfrak{p}_{1} \in L^{\frac{3}{2}}\left(\mathbb{T} ; L^{3}\left(\mathbb{R}^{3}\right)\right)$ with $\nabla \mathfrak{p}_{1} \in L^{\frac{3}{2}}\left(\mathbb{T} \times \mathbb{R}^{3}\right)^{3}$ such that

$$
v \cdot \nabla v+v \cdot \nabla w=\mathcal{P}_{H}(v \cdot \nabla v+v \cdot \nabla w)+\nabla \mathfrak{p}_{1} .
$$

As in the proof of Lemma 4.4.4, recall in particular (4.73) and (4.75), we further find that $w \cdot \nabla w \in L^{\frac{5}{4}}\left(\mathbb{T} \times \mathbb{R}^{3}\right)^{3} \cap L^{1}\left(\mathbb{T} \times \mathbb{R}^{3}\right)^{3}$. Since $q \in\left(1, \frac{5}{4}\right]$ we thus have $w \cdot \nabla w \in L^{q}\left(\mathbb{T} \times \mathbb{R}^{3}\right)^{3}$. Similarly, it follows that also $w \cdot \nabla v \in L^{q}\left(\mathbb{T} \times \mathbb{R}^{3}\right)^{3}$. Employing again Lemma 3.6.8, we find $\mathfrak{p}_{2} \in L^{q}\left(\mathbb{T} ; L^{\frac{3 q}{3-q}}\left(\mathbb{R}^{3}\right)\right)$ with $\nabla \mathfrak{p}_{2} \in L^{q}\left(\mathbb{T} \times \mathbb{R}^{3}\right)^{3}$ such that

$$
w \cdot \nabla w+w \cdot \nabla v=\mathcal{P}_{H}(w \cdot \nabla w+w \cdot \nabla v)+\nabla \mathfrak{p}_{2} .
$$

From (6.95) and (6.96) we infer

$$
u \cdot \nabla u=\mathcal{P}_{H}(u \cdot \nabla u)+\nabla \mathfrak{p}_{3}
$$

with $\mathfrak{p}_{3}:=\mathfrak{p}_{1}+\mathfrak{p}_{2}$ satisfying (6.91). By the same argument, the assumption (6.90) on $f$ implies that

$$
f=\mathcal{P}_{H} f+\nabla \mathfrak{p}_{4}
$$

with a pressure $\mathfrak{p}_{4}$ satisfying (6.91). Now consider some $\Phi \in C_{0}^{\infty}\left(\mathbb{T} \times \mathbb{R}^{3}\right)^{3}$. Recalling Lemma 3.2.3, we can apply the Helmholtz decomposition and obtain $\mathcal{P}_{H} \Phi \in L_{\sigma}^{r}\left(\mathbb{T} \times \mathbb{R}^{3}\right)$ for all $r \in(1, \infty)$. Moreover, by Lemma 3.6.8 $\left[\mathrm{Id}-\mathcal{P}_{H}\right] \Phi=\nabla \Pi$ with $\Pi \in \mathrm{X}_{\mathrm{P}}^{r}\left(\mathbb{T} \times \mathbb{R}^{3}\right)$ for all $r \in(1, \infty)$. Similarly, $\nabla \Phi=\mathcal{P}_{H} \nabla \Phi+\left[\operatorname{Id}-\mathcal{P}_{H}\right] \nabla \Phi$ with $\mathcal{P}_{H} \nabla \Phi \in L_{\sigma}^{r}\left(\mathbb{T} \times \mathbb{R}^{3}\right)^{3}$ and $\left[\mathrm{Id}-\mathcal{P}_{H}\right] \nabla \Phi=\nabla \Pi$ with $\Pi \in \mathrm{X}_{\mathrm{P}}^{r}\left(\mathbb{T} \times \mathbb{R}^{3}\right)^{3}$ for all $r \in(1, \infty)$. Consequently, since for $t \in \mathbb{T}$ the vector fields $v(t), w(t), \nabla v(t)$, and $\nabla w(t)$ all belong to some solenoidal function space $L_{\sigma}^{s}\left(\mathbb{R}^{3}\right)$ for an appropriately chosen $s \in(1, \infty)$, it is easy to verify that

$$
\begin{aligned}
\int_{\mathbb{T}} \int_{\mathbb{R}^{3}}-u \cdot \partial_{t} \Phi+\nabla u & : \nabla \Phi-\lambda \partial_{1} u \cdot \Phi \mathrm{d} x \mathrm{~d} t \\
& =\int_{\mathbb{T}} \int_{\mathbb{R}^{3}}-u \cdot \mathcal{P}_{H} \partial_{t} \Phi+\nabla u: \mathcal{P}_{H} \nabla \Phi-\lambda \partial_{1} u \cdot \mathcal{P}_{H} \Phi \mathrm{d} x \mathrm{~d} t \\
& =\int_{\mathbb{T}} \int_{\mathbb{R}^{3}}-u \cdot \partial_{t} \mathcal{P}_{H} \Phi+\nabla u: \nabla \mathcal{P}_{H} \Phi-\lambda \partial_{1} u \cdot \mathcal{P}_{H} \Phi \mathrm{d} x \mathrm{~d} t
\end{aligned}
$$

where the last equality is due to the fact that for sufficiently regular vector fields defined on the whole-space, for instance vector fields in $W^{1, s}\left(\mathbb{T} \times \mathbb{R}^{3}\right)^{3}, s \in(1, \infty)$, the Helmholtz projection $\mathcal{P}_{H}$ commutes with $\nabla$ and $\partial_{t}$. This is an easy consequence of, for example, Lemma 2.3.5. Clearly $\mathcal{P}_{H} \Phi \in W_{0, \sigma}^{1, s}\left(\mathbb{T} \times \mathbb{R}^{3}\right)$ for all $s \in(1, \infty)$. Thus $\mathcal{P}_{H} \Phi$ can be approximated by elements $\left\{\Psi_{n}\right\}_{n=1}^{\infty}$ from $C_{0, \sigma}^{\infty}\left(\mathbb{T} \times \mathbb{R}^{3}\right)$ in any $W^{1, s}\left(\mathbb{T} \times \mathbb{R}^{3}\right)$-norm. We can thus utilize that $u$ is a weak solution to (6.1), that is, it satisfies (6.4) for all $\Psi_{n}$, to 


\section{Weak solutions}

conclude from (6.99) that

$$
\begin{aligned}
\int_{\mathbb{T}} \int_{\mathbb{R}^{3}}-u & \cdot \partial_{t} \Phi+\nabla u: \nabla \Phi-\lambda \partial_{1} u \cdot \Phi \mathrm{d} x \mathrm{~d} t \\
& =\iint_{\mathbb{T}} f \cdot \mathcal{P}_{H} \Phi-(u \cdot \nabla u) \cdot \mathcal{P}_{H} \Phi \mathrm{d} x \mathrm{~d} t \\
& =\int_{\mathbb{T}} \int_{\mathbb{R}^{3}} f \cdot \Phi-(u \cdot \nabla u) \cdot \Phi-f \cdot \nabla \Pi+(u \cdot \nabla u) \cdot \nabla \Pi \mathrm{d} x \mathrm{~d} t \\
& =\int_{\mathbb{T}} \int_{\mathbb{R}^{3}} f \cdot \Phi-(u \cdot \nabla u) \cdot \Phi-\nabla \mathfrak{p}_{4} \cdot \nabla \Pi+\nabla \mathfrak{p}_{3} \cdot \nabla \Pi \mathrm{d} x \mathrm{~d} t \\
& =\int_{\mathbb{T}} \int_{\mathbb{R}^{3}} f \cdot \Phi-(u \cdot \nabla u) \cdot \Phi-\nabla\left(\mathfrak{p}_{4}-\mathfrak{p}_{3}\right) \cdot \Phi \mathrm{d} x \mathrm{~d} t .
\end{aligned}
$$

Since $\mathfrak{p}:=\mathfrak{p}_{4}-\mathfrak{p}_{3}$ satisfies (6.91), we deduce (6.92).

Assume now that $\lambda>0$. We then obtain from Lemma 4.4.4 that $v \in \mathrm{X}_{\sigma, \text { Oseen }}^{q}\left(\mathbb{R}^{3}\right)$. It follows that $v \in L^{\frac{2 q}{2-q}}\left(\mathbb{R}^{3}\right)^{3}$, which by Hölders inequality implies that $v \cdot \nabla v \in L^{q}\left(\mathbb{R}^{3}\right)^{3}$ and also $v \cdot \nabla w \in L^{q}\left(\mathbb{T} \times \mathbb{R}^{3}\right)^{3}$. We can now repeat the proof above to obtain a pressure $\mathfrak{p}$ that satisfies (6.94). More specifically, since $v \cdot \nabla v+v \cdot \nabla w \in L^{q}\left(\mathbb{R}^{3}\right)^{3}$ we can find a pressure term $\mathfrak{p}_{1}$ satisfying (6.94) such that (6.95) holds. Consequently, we obtain (6.97) with $\mathfrak{p}_{3}$ also satisfying (6.94). The rest of the proof follows as above. 


\section{Bibliography}

[1] R. A. Adams. Sobolev spaces. Academic Press, New York-London, 1975. 20

[2] K. Babenko. On stationary solutions of the problem of flow past a body of a viscous incompressible fluid. Math. USSR, Sb., 20:1-25, 1973. 13

[3] F. Bruhat. Distributions sur un groupe localement compact et applications à l'étude des représentations des groupes p-adiques. Bull. Soc. Math. Fr., 89:43-75, 1961. 27

[4] K. de Leeuw. On $L_{p}$ multipliers. Ann. Math. (2), 81:364-379, 1965. 11, 24, 36

[5] R. Edwards and G. Gaudry. Littlewood-Paley and multiplier theory. BerlinHeidelberg-New York: Springer-Verlag, 1977. 36

[6] R. Farwig. An $L^{q}$-analysis of viscous fluid flow past a rotating obstacle. Tohoku Math. J. (2), 58(1):129-147, 2006. 53, 54, 59

[7] R. Farwig, G. P. Galdi, and M. Kyed. Asymptotic structure of a Leray solution to the Navier-Stokes flow around a rotating body. Pac. J. Math., 253(2):367-382, 2011. 15,107

[8] R. Farwig and T. Hishida. Leading term at infinity of steady Navier-Stokes flow around a rotating obstacle. Math. Nachr., 284(16):2065-2077, 2011. 15, 107

[9] R. Farwig, T. Hishida, and D. Müller. $L^{q}$-theory of a singular winding integral operator arising from fluid dynamics. Pac. J. Math., 215(2):297-312, 2004. 53, 54, 59

[10] R. Farwig and H. Sohr. Weighted estimates for the Oseen equations and the NavierStokes equations in exterior domains. In Theory of the Navier-Stokes equations, volume 47 of Ser. Adv. Math. Appl. Sci., pages 11-30. World Sci. Publ., River Edge, NJ, 1998. 13

[11] R. Finn. An energy theorem for viscous fluid motions. Arch. Ration. Mech. Anal., 6:371-381, 1960. 129

[12] R. Finn. Estimates at infinity for stationary solutions of the Navier-Stokes equations. Bull. Math. Soc. Sci. Math. Phys. R.P.R., n. Ser., 3(51):387-418, 1960. 13

[13] R. Finn. On the exterior stationary problem for the Navier-Stokes equations, and associated perturbation problems. Arch. Ration. Mech. Anal., 19:363-406, 1965. 13 


\section{BIBLIOGRAPHY}

[14] G. Galdi and H. Sohr. Existence and uniqueness of time-periodic physically reasonable Navier-Stokes flow past a body. Arch. Ration. Mech. Anal., 172(3):363-406, 2004. 6,7

[15] G. P. Galdi. On the asymptotic structure of $D$-solutions to steady Navier-Stokes equations in exterior domains. In Mathematical problems relating to the NavierStokes equation, volume 11 of Ser. Adv. Math. Appl. Sci., pages 81-104. World Sci. Publ., River Edge, NJ, 1992. 13

[16] G. P. Galdi. On the asymptotic properties of Leray's solutions to the exterior steady three-dimensional Navier-Stokes equations with zero velocity at infinity. In Degenerate diffusions (Minneapolis, MN, 1991), volume 47 of IMA Vol. Math. Appl., pages 95-103. Springer, New York, 1993. 81

[17] G. P. Galdi. An introduction to the mathematical theory of the Navier-Stokes equations. Vol. I: Linearized steady problems. Springer Tracts in Natural Philosophy. 38. New York: Springer-Verlag, 1994. 20, 21, 29, 31, 45, 46, 56, 57, 63, 92, 93, 99, 101, 102, 103, 104, 136, 139

[18] G. P. Galdi. An introduction to the mathematical theory of the Navier-Stokes equations. Vol. II: Nonlinear steady problems. Springer Tracts in Natural Philosophy. 39. New York: Springer-Verlag, 1994. 21, 83, 84, 85, 96, 98, 99, 113, 114, 129

[19] G. P. Galdi. An introduction to the Navier-Stokes initial-boundary value problem. In Fundamental directions in mathematical fluid mechanics, Adv. Math. Fluid Mech., pages 1-70. Birkhäuser, Basel, 2000. 3, 133

[20] G. P. Galdi. On the motion of a rigid body in a viscous liquid: a mathematical analysis with applications. In Handbook of mathematical fluid dynamics, Vol. I, pages 653-791. North-Holland, Amsterdam, 2002. 7, 53

[21] G. P. Galdi. An introduction to the mathematical theory of the Navier-Stokes equations. Steady-state problems. 2nd ed. New York: Springer, 2011. 3, 53, 76, 77, 106

[22] G. P. Galdi and M. Kyed. A simple proof of $L^{q}$-estimates for the steady-state Oseen and Stokes equations in a rotating frame, Part II: Weak solutions. To appear in Proc. Amer. Math. Soc., 2011. 59

[23] G. P. Galdi and M. Kyed. Steady-state Navier-Stokes flows past a rotating body: Leray solutions are physically reasonable. Arch. Ration. Mech. Anal., 200(1):21-58, 2011. 103

[24] G. P. Galdi and M. Kyed. A simple proof of $L^{q}$-estimates for the steady-state Oseen and Stokes equations in a rotating frame. Part I: Strong solutions. Proc. Amer. Math. Soc., 141:573-583, 2013. 59 
[25] G. P. Galdi and A. L. Silvestre. Existence of time-periodic solutions to the NavierStokes equations around a moving body. Pac. J. Math., 223(2):251-267, 2006. 7, $8,9,111$

[26] G. P. Galdi and A. L. Silvestre. The steady motion of a Navier-Stokes liquid around a rigid body. Arch. Ration. Mech. Anal., 184(3):371-400, 2007. 103

[27] G. P. Galdi and A. L. Silvestre. On the motion of a rigid body in a Navier-Stokes liquid under the action of a time-periodic force. Indiana Univ. Math. J., 58(6):28052842, 2009. 8

[28] L. Grafakos. Classical Fourier analysis. 2nd ed. New York, NY: Springer., 2008. $22,36,37,92$

[29] L. Grafakos. Modern Fourier analysis. 2nd ed. New York, NY: Springer., 2009. 52

[30] J. G. Heywood. The Navier-Stokes equations: On the existence, regularity and decay of solutions. Indiana Univ. Math. J., 29:639-681, 1980. 5

[31] T. Hishida. $L^{q}$ estimates of weak solutions to the stationary Stokes equations around a rotating body. J. Math. Soc. Japan, 58(3):743-767, 2006. 59

[32] E. Hopf. Über die Anfangswertaufabe für die hydrodynamischen Grundgleichungen. Math. Nachr., 4:213-231, 1951. 4, 128, 133

[33] K. Kang, H. Miura, and T.-P. Tsai. Asymptotics of small exterior Navier-Stokes flows with non-decaying boundary data. Preprint. 7, 14

[34] S. Kaniel and M. Shinbrot. A reproductive property of the Navier-Stokes equations. Arch. Rational Mech. Anal., 24:363-369, 1967. 4

[35] A. Korolev and V. Šverák. On the large-distance asymptotics of steady state solutions of the Navier-Stokes equations in 3D exterior domains. Ann. Inst. Henri Poincaré, Anal. Non Linéaire, 28(2):303-313, 2011. 13, 129

[36] H. Kozono and M. Nakao. Periodic solutions of the Navier-Stokes equations in unbounded domains. Tohoku Math. J. (2), 48(1):33-50, 1996. 5, 6

[37] S. Kračmar, Š. Nečasová, and P. Penel. $L^{q}$-approach to weak solutions of the Oseen flow around a rotating body. In Parabolic and Navier-Stokes equations. Part 1, volume 81 of Banach Center Publ., pages 259-276. Polish Acad. Sci. Inst. Math., Warsaw, 2008. 59

[38] S. Kracmar, S. Necasová, and P. Penel. $L^{q}$-approach of weak solutions to stationary rotating Oseen equations in exterior domains. Q. Appl. Math., 68(3):421-437, 2010. 59

[39] M. Kyed. On the asymptotic structure of a Navier-Stokes flow past a rotating body. To appear in J. Math. Soc. Japan, 2011. 15, 107 


\section{BIBLIOGRAPHY}

[40] L. Landau. A new exact solution of Navier-Stokes equations. C. R. (Dokl.) Acad. Sci. URSS, n. Ser., 43:286-288, 1944. 14

[41] J. Leray. Étude de diverses équations intégrales non linéaires et de quelques problèmes que pose l'hydrodynamique. J. Math. Pures Appl., 12:1-82, 1933. 3

[42] J. Leray. Sur le mouvement d'un liquide visqueux emplissant l'espace. Acta Math., 63(1):193-248, 1934. 3, 128, 133

[43] J. Marcinkiewicz. Sur les multiplicateurs des séries de Fourier. Studia math., Lwów,, 8:78-91, 1939. 22

[44] P. Maremonti. Existence and stability of time-periodic solutions to the NavierStokes equations in the whole space. Nonlinearity, 4(2):503-529, 1991. 5, 6

[45] P. Maremonti. Some theorems of existence for solutions of the Navier-Stokes equations with slip boundary conditions in half-space. Ric. Mat., 40(1):81-135, 1991. 5, 6

[46] P. Maremonti and M. Padula. Existence, uniqueness and attainability of periodic solutions of the Navier-Stokes equations in exterior domains. Zap. Nauchn. Sem. S.-Peterburg. Otdel. Mat. Inst. Steklov. (POMI), 233(Kraev. Zadachi Mat. Fiz. i Smezh. Vopr. Teor. Funkts. 27):142-182, 257, 1996. 5

[47] T. Miyakawa and Y. Teramoto. Existence and periodicity of weak solutions of the Navier-Stokes equations in a time dependent domain. Hiroshima Math. J., 12(3):513-528, 1982. 4

[48] H. Morimoto. On existence of periodic weak solutions of the Navier-Stokes equations in regions with periodically moving boundaries. J. Fac. Sci., Univ. Tokyo, Sect. I A, 18:499-524, 1972. 4

[49] G. Prodi. Qualche risultato riguardo alle equazioni di Navier-Stokes nel caso bidimensionale. Rend. Sem. Mat. Univ. Padova, 30:1-15, 1960. 4

[50] G. Prouse. Soluzioni periodiche dell'equazione di Navier-Stokes. Atti Accad. Naz. Lincei Rend. Cl. Sci. Fis. Mat. Natur. (8), 35:443-447, 1963. 4

[51] G. Prouse. Soluzioni quasi-periodiche dell'equazione differenziale di Navier-Stokes in due dimensioni. Rend. Sem. Mat. Univ. Padova, 33:186-212, 1963. 4

[52] J. Serrin. A note on the existence of periodic solutions of the Navier-Stokes equations. Arch. Rational Mech. Anal., 3:120-122, 1959. 3

[53] A. L. Silvestre. Existence and uniqueness of time-periodic solutions with finite kinetic energy for the Navier-Stokes equations in $\mathbb{R}^{3}$. Nonlinearity, 25(1):37-55, 2012. 7,81 
[54] E. M. Stein. Singular integrals and differentiability properties of functions. Princeton, N.J.: Princeton University Press, 1970. 22

[55] A. Takeshita. On the reproductive property of the 2-dimensional Navier-Stokes equations. J. Fac. Sci. Univ. Tokyo Sect. I, 16:297-311 (1970), 1969. 4

[56] R. Temam. Navier-Stokes equations. Theory and numerical analysis. Rev. ed. Amsterdam-New York-Oxford: North-Holland Publishing Company, 1979. 126

[57] Y. Teramoto. On the stability of periodic solutions of the Navier-Stokes equations in a noncylindrical domain. Hiroshima Math. J., 13:607-625, 1983. 4

[58] H. Weinberger. Variational properties of steady fall in Stokes flow. J. Fluid Mech., $52: 321-344,1972.8$

[59] H. F. Weinberger. On the steady fall of a body in a Navier-Stokes fluid. In Partial differential equations (Proc. Sympos. Pure Math., Vol. XXIII, Univ. California, Berkeley, Calif., 1971), pages 421-439. Amer. Math. Soc., Providence, R. I., 1973. 8

[60] M. Wiegner. The Navier-Stokes equations - a neverending challenge? Jahresber. Dtsch. Math.-Ver., 101(1):1-25, 1999. 3

[61] M. Yamazaki. The Navier-Stokes equations in the weak- $L^{n}$ space with timedependent external force. Math. Ann., 317(4):635-675, 2000. 6, 7

[62] V. Yudovich. Periodic motions of a viscous incompressible fluid. Sov. Math., Dokl., 1:168-172, 1960. 4 


\section{Index}

$A_{\text {Oseen }}, 45$

$A_{\text {Stokes }}, 45$

$\mathrm{A}_{T P}, 44$

asymptotic expansion, 83, 93, 100

Bochner-Lebesgue space, 22

Bochner-Sobolev space, 110

Bogovskiü-kernel, 30

$C^{k}(\Omega), C^{\infty}(\Omega), C_{0}^{k}(\Omega), C_{0, \sigma}^{\infty}(\Omega), 19,20$

$C^{\infty}\left(\mathbb{R}^{3} \times \mathbb{T}\right), C_{0, \sigma}^{\infty}\left(\mathbb{R}^{3} \times \mathbb{T}\right), 110$

$C^{\infty}(G), C_{0}^{\infty}(G), C_{0, \sigma}^{\infty}(G), 27,29$

$\mathcal{C}_{\text {per }}, 36$

$\mathcal{D}^{\prime}\left(\mathbb{R}^{3} \times \mathbb{T}\right), 134$

$D^{m, q}(\Omega),|\cdot|_{m, q}, 19$

$D_{0}^{-m, q}(\Omega),|\cdot|_{-m, q}, 20$

$D_{0}^{m, q}(\Omega), D_{0, \sigma}^{m, q}(\Omega), 19,20$

differentiable structure on $G, 27$

distributional derivative, 28

dual group, 18

energy equation, 72

$\mathcal{F}, 18,33$

flow past rotating body, 53 asymptotic expansion, 103

Fourier transform, 18, 33, 110

$\Gamma_{\mathrm{SOR}}^{\lambda, k}, 86$

$\Gamma_{\mathrm{O}}^{\lambda}, 92$

$\Gamma^{\perp}, 88$

grad, 51

$\mathscr{G}^{q, r}(G), 62$

$\mathscr{G}^{q}(\Omega), 21$

$\mathscr{G}^{q}(G), 32$

$G, \widehat{G}, 27,33$
Haar measure, 28

Hardy inequality, 21

Helmholtz projection, 21, 32

$\mathrm{K}, 55$

kinetic energy, 102, 129

$L^{q, r}(G), L_{\sigma}^{q, r}(G),\|\cdot\|_{q, r}, 62$

$L^{q}(S ; X), L^{\infty}(S ; X), 22$

$L^{q}(\Omega), L_{\sigma}^{q}(\Omega),\|\cdot\|_{q}, 19,20$

$L^{q}(G), L_{\sigma}^{q}(G),\|\cdot\|_{q}, 28,29$

$L_{\perp}^{q}(G), L_{\sigma, \perp}^{q}(G), 42$

$L_{\perp}^{q}(\mathbb{T} ; X), 112$

$L_{w}^{\frac{1}{2}}\left(\mathbb{R}^{3}\right), 131$

Marcinkiewicz multiplier theorem, 22

$\mathcal{P}_{H}, 21,32$

$\mathcal{P}, \mathcal{P}_{\perp}, 41,43,111$

$\pi, 27$

physically reasonable weak solution, 74

$Q(t), 54$

$\mathscr{S}(G), 27,33$

$\mathscr{S}^{\prime}(G), 28$

Schwartz-Bruhat space, 27, 33

Sobolev embedding, 20

Sobolev space, 19, 29

solenoidal, 20, 29

strong solution, 61

$\mathbb{T}, 18$

tempered distributions, 28

torus group, 18

transference of multipliers, 36

$W^{1,2, q}(G), W_{\sigma}^{1,2, q}(G),\|\cdot\|_{1,2, q}, 29$ 
$W^{\alpha, 2}(\mathbb{T} ; H),\|\cdot\|_{W^{\alpha, 2}(\mathbb{T} ; H)}, 110$

$W^{k, q}(G),\|\cdot\|_{k, q}, 29$

$W^{m, q}(\Omega),\|\cdot\|_{m, q}, 19$

$W_{0, \sigma}^{m, q}(\Omega), 20$

$W_{\sigma, \perp}^{1,2, q, r}(G),\|\cdot\|_{1,2, q, r}, 62$

$W_{\sigma, \perp}^{1,2, q}(G), 42$

weak solution, 111

$\mathrm{X}_{\sigma, \text { Oseen }}^{q, r}\left(\mathbb{R}^{3}\right),\|\cdot\|_{\mathrm{X}_{\sigma, \text { Oseen }}^{q, r}\left(\mathbb{R}^{3}\right)}, 62$

$\mathrm{X}_{\sigma, \text { Oseen }}^{q}\left(\mathbb{R}^{3}\right),\|\cdot\|_{q, \lambda, \text { Oseen }}, 45$

$\mathrm{X}_{\mathrm{P}}^{q, r}(G),\|\cdot\|_{\mathrm{X}_{\mathrm{P}}^{q, r}(G)}, 62$

$\mathrm{X}_{\mathrm{P}}^{q}(G),\|\cdot\|_{\mathrm{X}_{\mathrm{P}}^{q}(G)}, 51$

$\mathrm{X}_{\sigma, \text { Stokes }}^{q}\left(\mathbb{R}^{3}\right),\|\cdot\|_{q, \text { Stokes }}, 46$ 